\title{
Magnetic Survey of D-Area Oil Basin Waste Unit
}

by

R. J. Cumbest

Westinghouse Savannah River Company

Savannah River Site

Aiken, South Carolina 29808

D. Marcy

J. Hango

S. Bently

B. Hunter

B. Cain

DOE Contract No. DE-A C09-89SR18035

This paper was prepared in connection with work done under the above contract number with the U.S.

Department of Energy. By acceptance of this paper, the publisher and/or recipient acknowledges the U.S.

Government's right to retain a nonexclusive, royalty-free license in and to any copyright covering this paper, along with the right to reproduce and to authorize others to reproduce all or part of the copyrighted paper. 


\section{DISCLAIMER}

This report was prepared as an account of work sponsored by an agency of the United States Government. Neither the United States Government nor any agency thereof, nor any of their employees, makes any warranty, express or implied, or assumes any legal liability or responsibility for the accuracy, completeness, or usefulness of any information, apparatus, product, or process disclosed, or represents that its use would not infringe privately owned rights. Reference herein to any specific commercial product, process, or service by trade name, trademark, manufacturer, or otherwise does not necessarily constitute or imply its endorsement, recommendation, or favoring by the United States Government or any agency thereof. The views and opinions of authors expressed herein do not necessarily state or reflect those of the United States Government or any agency thereof.

This report has been reproduced directly from the best available copy.

Available to DOE and DOE contractors from the Office of Scientific and Technical Information, P.O. Box 62, Oak Ridge, TN 37831; prices available from (615) 576-8401.

Available to the public from the National Technical Information Service, U.S. Department of Commerce, 5285 Port Royal Road, Springfield, VA 22161. 


\section{DISCLAIMER}

Portions of this document may be illegible in electronic image products. Images are produced from the best available original document. 


\section{Magnetic Survey of D-Area Oil Basin Waste Unit}

\section{INTRODUCTION AND SCOPE}

The D-Area Oil Basin RCRA Waste Unit is located north of D-Area on Savannah River Site (Fig. 1). This Waste Unit was known, based on aerial photography (Fig. 2) and other historical data, to be the location for one or more trenches used for disposal of oil in steel drums and other refuse. In order to define the location of possible trenches on the site and to assess the possibility of the presence of additional buried objects a magnetic survey was conducted by the Environmental Monitoring Section/Groundwater Group during July, 1993, at the request of the Environmental Restoration Department. Prior to the conduct of the magnetic survey a Ground Penetrating Radar survey of the site consisting of several lines identified several areas of disturbed soil. Based on these data and other historical information the general orientation of the trenches could be inferred. The magnetic survey consists of a rectangular grid over the waste unit designed to maximize resolution of the trench edges. This report describes the magnetic survey of the D-Area Oil Basin Waste Unit.

\section{Magnetic Survey Concepts}

The magnitude of the magnetic field measured near the earth's surface is influenced by several factors. The main contribution to the total intensity is due to the internal magnetic field of the earth produced in the vicinity of the earth's core. However, this field is modified by the solar wind of charged particles produced by electromagnetic activity on the sun. This solar wind distorts the magnetic field produced by the earth into a tear drop shape with the blunt end pointing toward the sun. More importantly, the effect of the solar wind is to produce time variations in the total field. The earth's rotation, in effect, introduces a diurnal variation with a twenty four hour period. Also, superimposed on this well defined twenty four hour cycle are variations with no well defined period produced by 


\section{D-Area Oil Basin Magnetic Survey}

the chaotic nature of the suns electromagnetic activity. Typically these variations manifest themselves as micropulsations on the order of less than $10 \mathrm{nT}$, but significantly larger variations can be produced by magnetic storms on the sun (i.e. sunspots). The net result of these variations is illustrated in figure 3 .

The earth's magnetic field is also modified by features near the surface that produce localized spatial variations, or anomalies, in the field intensity. The use of magnetic techniques in searching for buried metallic objects is a consequence of the ability of iron and iron alloys to cause relatively large amplitude anomalies. These anomalies are produced by two effects. Some materials have properties that allow them to become magnetized in the presence on an external magnetic field. This process is called induction. The external magnetic field is the inducing field and the field produced by the material the induced field. The total field (B)now becomes the sum of the inducing external field ( $\overrightarrow{\mathbf{H}}$ ) and the field produced by the material $\left(\vec{H}^{\prime}\right)$. This relationship can be expressed:

$$
\overrightarrow{\mathbf{Z}}=\overrightarrow{\mathbf{H}}+\overrightarrow{\mathbf{H}^{\prime}}
$$

In addition some materials possess a magnetic field independent from any external source. This field is typically acquired at some time in the past history of the material and is called remnant magnetization. For most magnetic survey applications the magnitude of the induced field is assumed to be much greater than the remnant magnetization and this assumption has been shown to be valid in the specific case of standard 55 gallon metal drums (Gilkeson and others, 1992).

In reality magnetic fields are always manifested as dipoles. That is north and south magnetic poles occur in pairs to produce dipole fields. A field line representation of a dipole and monopole field are illustrated in figure 4. In this type of representation the density of the field lines is proportional to the field magnitude. To a first approximation, the magnetic field produced near the core of the earth can be considered a single dipole 
with the magnetic field lines impinging the surface of the earth at 90 degrees at the north and south magnetic poles and parallel to the surface of the earth at the magnetic equators.

The geometric relationship of the magnetic field lines to the geographic poles is expressed by magnetic inclination and declination. Declination describes the angular relationship of the component of the field line parallel to the surface of the earth, to geographic north. The magnetic inclination expresses the angular relationship between the magnetic field lines and the horizontal (Fig. 5). Although, these angular relationships change slowly due to poorly understood long term variations, the declination in the vicinity of SRS is about 4 degrees to the west and the inclination about 65 degrees.

Although inducing bodies usually possess highly irregular shapes and sizes they can be usefully modeled as dipoles or arrays of dipoles. In some instances, if the area of consideration is near one end of a dipole relative to the dipole separation, the local field can be considered to be produced by a single pole and approximates monopole behavior. The anomalous fields produced by a dipole and monopole for a magnetic inclination of 60 degrees in the northern hemisphere (i.e. conditions very similar to SRS) and their resultant effect on the total magnetic field measured near the body are illustrated in figure 4 . Notice that the anomalous field lines on the north side of the dipole are opposite in sense to the ambient field therefore reducing the field strength in this location. In contrast the anomalous field lines on the south side are in the same sense resulting in an increased field strength. The monopole exhibits similar behavior although not as extreme. This phenomena is the explanation for the asymmetrical shaped magnetic highs and lows produced by inducing bodies at mid-latitudes on the earth. Figure 6 illustrates typical magnetic profiles that would be observed at the latitude of SRS over monopoles and dipoles in addition to a generalized contour representation of a dipole, with illustrative profiles across the contours.

Generalized magnetic field intensity profiles across more complicated shapes for an inclination of 60 degrees are illustrated in figure 7. These profiles all exhibit the typical 


\section{D-Area Oil Basin Magnetic Survey}

asymmetrical signatures along the north - south profiles with magnetic highs occurring over southern portions of the bodies.

The previous discussion has been presented in the context of the intensity of the total magnetic field. However, another measurement which can be very useful in interpreting magnetic anomalies is the vertical magnetic gradient, which changes much more rapidly with distance than the total field. This effect results in edge enhancement of anomalies and more effective anomaly separation (Fig. 8). The vertical magnetic gradient can be determined by making measurements of the total magnetic intensity at two known elevations. The vertical gradient is then determined by the difference between the two measurements divided by their separation. The two measurements can be made by a single sensor instrument by moving the sensor between the two measurements or by an instrument with two separated sensors.

In order to conduct a magnetic survey magnetic data are collected at discrete intervals either along a traverse or over a 2 dimensional grid. In practice, data for 2-D grids are usually collected as multiple traverses. Traverse data can be conveniently displayed as magnetic intensity profiles. 2-D gridded data are usually contoured and displayed as contour diagrams. Displayed in this fashion these data can be qualitatively interpreted by comparing them with profiles or contours that would be predicted from bodies of known shape and size as presented earlier.

In addition to qualitative information, profiles and contour diagrams may also be used for quantitative analysis if the magnetic response of specific objects is known. Due to their widespread use in the past as containers for industrial materials 55 gallon steel drums are common targets associated with characterization of waste sites. Gilkeson and others (1992) have evaluated the magnetic response from single and multiple drums buried at different depths. The magnetic response is provided by a model calibrated from actual measurements on a 55 gallon drum. These anomalies were calculated for a total field intensity of $53220 \mathrm{nT}$ and inclination of 70 degrees. Conditions very similar to SRS. 
Figure 9 illustrates model total field and vertical gradient profiles over a single drum, in vertical position, with the drum top at different depths below the sensor. If single, deeply buried drums are the target, these profiles make clear the need to locate the sensor near the ground surface in order to minimize the distance between the sensor and drum. For sensor - drum distances greater than about $20 \mathrm{ft}$ the anomaly amplitude has decreased to values that may not be discernible from noise.

Figure 10 illustrates the contour representation of an anomaly for a single drum with its top located $10 \mathrm{ft}$ below the sensor. Also shown in figure 10 are the station locations of a typical $5 \mathrm{ft}$ by $5 \mathrm{ft}$ grid over the anomaly. Note that a misfortunate placement of a $20 \mathrm{ft}$ by $20 \mathrm{ft}$ grid may fail to detect the anomaly at all and that a badly placed $10 \mathrm{ft}$ by $10 \mathrm{ft}$ grid would only reveal a maximum amplitude on the order of $25 \mathrm{nT}$ which may also not be discernible from noise and at best would offer poor resolution concerning the drum location.

Figure 11 illustrates the contour representation of a total field magnetic anomaly for a cache of 5 drums located in a $12 \mathrm{ft}$ by $12 \mathrm{ft}$ area with the drum tops $10 \mathrm{ft}$ below the sensor. Note again the a $20 \mathrm{ft}$ by $20 \mathrm{ft}$ grid may fail to detect the anomaly. A $10 \mathrm{ft}$ by $10 \mathrm{ft}$ grid would crudely resolve the anomaly. However, Gilkeson and other's (1992) modeling demonstrated that at a depth of $10 \mathrm{ft}$ if the center drum were removed the drums on the corners would be resolved into individual anomalies. If this type of information is required from the magnetic survey then $5 \mathrm{ft}$ grid node separations would be needed to fully resolve the individual anomalies associated with each drum.

In addition to the above considerations other factors enter into the design of an effective magnetic survey. Although smaller grid spacings allow better resolution of anomalies they are also labor intensive. Placing the sensor near the ground makes it much more sensitive to small, shallow sources such as metallic trash or magnetite variations in the soil. This is especially a problem for gradiometer surveys because these effects are amplified. In addition gradiometers are most effective when the sensor separation is $1 / 5$ or 


\section{D-Area Oil Basin Magnetic Survey}

less the distance to the target. If the sensors are placed near the ground this condition may not be met for shallow targets. A careful consideration of what information is needed from the magnetic survey and the resources available will determine which of the aspects discussed above are most important.

\section{Description of Survey Site}

A planimetric map of D-Area Oil Basin Waste Unit is presented in Figure 12a along with the planimetric information overlain onto the aerial photograph in Figure $12 \mathrm{~b}$. The reference for this map is the Planimetric Map of " $D$ " Area Oil Seepage Basin prepared by Cranston, Robertson and Whitehurst, P.C., 1988. A dirt road runs southwestnortheast along the northwest side of the Waste Unit boundary. Groundwater monitoring wells (DOB1 - 4) occur adjacent to the waste unit boundaries. The boundaries of the waste site are delimited by four orange spherical markers at the corners, in addition to a metal fence which surrounds the site. In some areas only the metal fence posts remain. The most prominent objects at the Waste Site are a double row of wooden posts that mark the position of a berm. These wooden posts supposedly delimit the location of a burial trench. Also located on the waste site are several small concrete pads which occur parallel to and near the northwest site boundary. In addition to these objects, a metal wheel borrow was located at the southern corner of the waste site.

Previous to the magnetic survey several borings were made on the waste site for soil sampling. The locations of these borings are shown on Figures $12 \mathrm{a}$ and $12 \mathrm{~b}$ and the grid coordinates for each boring along with findings are listed in Table I.. Most of these borings encountered no metal objects. However, two of the borings (DOSB-03A and DOSB-1A) encountered metal wire and boring DOSB-03B penetrated a metal drum at 5 $7 \mathrm{ft}$. depth. Also, previous to the magnetic survey a ground penetrating radar survey was conducted at the waste unit (Sexton and Perkle, 1992). This survey identified several areas 
of disturbed soil. The ground penetrating radar survey lines are shown in figure 13 for reference.

TABLE I. Soil Borings at D - Area Oil Basin

\begin{tabular}{|l|c|c|l|}
\hline Soil Boring & $\mathbf{X}$ & $\mathbf{Y}$ & Findings \\
\hline DOSB-03A & 81.52 & 38.48 & Metal \& Wire \\
\hline DOSB-03B & 75.60 & 40.63 & Drum @ 5-7 ft down \\
\hline DOSB-03 & 69.61 & 39.78 & nothing \\
\hline DOSB $-02 A$ & 49.04 & 38.11 & Clean White Sand \\
\hline DOSB-02 & 43.35 & 38.69 & nothing \\
\hline DOSB -01 & 15.82 & 38.85 & nothing \\
\hline DOSB-01A by DOB4 & 5.64 & 39.57 & Asphault, metal wire \\
\hline
\end{tabular}

\section{MAGNETIC SURVEY}

\section{Survey and Analytical Techniques}

The grid established for the magnetic survey of D Area Oil Basin (fig. 14) was defined by 1 meter station intervals along lines spaced 3 meters apart to produce a rectangular grid node pattern. The grid was established to give coverage to the majority of the area enclosed by the fence and orange waste unit marker balls. Dense vegetation at each end of the waste unit either precluded coverage or forced the grid pattern to be altered to a $2 \times 2$ meter square. The survey lines were oriented perpendicular to the suspected trenches in order to maximize the resolution of the trench edges. The grid station locations were established with a transit to mark 90 degree angles and a cord marked at 1 meter intervals to determine distances.

Magnetic data were collected with an EG\&G Geometrics G-856 Gradiometer with the top sensor at 1.22 meters $(4 \mathrm{ft}$ ) from ground level and the bottom sensor 0.61 meters $(2 \mathrm{ft})$ from ground level. This configuration places the sensors near the ground to maximize the sensitivity to small near surface or deeply buried objects. This will not optimize the gradiometer function due to the short distance between the sensors and any possible large anomaly sources present in the suspected trench. However, the near surface 
sensitivity was deemed more important in order to detect all buried objects that may be present. This instrument has a nominal accuracy of $0.1 \mathrm{nT}$. However, high magnetic gradients $(>600 \mathrm{nT} / \mathrm{m})$ will cause the signal to be sharply degraded. In most cases this will cause the accuracy to deteriorate to $1 \mathrm{nT}$. More severe degradation can result in significant accuracy losses resulting in poor data very near large metallic objects. As a QA/QC check and in order to account for diurnal variation a single station was occupied several times during the survey.

The data were downloaded from the magnetometer through a RS-232 port to an IBM PC Model 80. After downloading, the data were assigned coordinates and diurnally corrected with MAGLOC, software provided by EG\&G. Further data display and processing, including gridding and contouring were accomplished with software provided by GEOSOFT Inc. Gridding of the data was accomplished with RANGRD, which fits a minimal curvature surface to the gridded data. Nonlinear filtering to remove data spikes caused by poor signal quality was accomplished with XYZFLT. Magnetic gradients were calculated by subtracting the top sensor reading from the bottom sensor and dividing by their separation. Since the top and bottom sensor readings are taken at a relatively short time scale (approximately 3 seconds) relative to diurnal variations the gradient is calculated from the data before diurnal correction. The data for the survey along with daily diurnal variations are tabulated in the Appendix.

\section{Magnetic Data Set}

Several anomalies are present in the magnetic data (Figs. 15-17). The most prominent magnetic anomalies occur as linear dipoles defined by parallel magnetic highs and lows trending across the waste unit in the approximate locations of the linear features seen in the aerial photographs (Fig. 18). The linear anomaly aligned approximately along the 60 meter $y$ coordinate is the largest in amplitude and displays a remarkable amount of lateral continuity, consisting of three discrete but continuous features. In contrast the 


\section{D-Area Oil Basin Magnetic Survey}

linear anomaly that occurs aligned approximately along the 40 - 50 meter y coordinate in the location defined by the trench marker posts displays limited lateral continuity and appears to be composed of aligned but isolated small dipole anomalies.

In addition to the prominent linear anomalies discussed above, isolated dipoles occur approximately centered at grid coordinates $(42,15),(67,26)$, and $(83,26)$. These anomalies occur in both the top and bottom sensor data sets and are therefore significant. Other minor anomalies occur in the data set including magnetic lows and isolated dipoles around the perimeter of the waste unit.

\section{DISCUSSION}

The two linear anomalies discussed above appear to mark the locations of trenches in which iron bearing objects were buried. The outlines of the inferred trench boundaries, based on the vertical gradient data are illustrated in Figure 19. The southern most trench anomaly exhibits a high degree of lateral continuity consistent with this trench containing a large number of small but continuously distributed metal objects or a few single long metal objects. The northern most trench does not exhibit this continuity and appears to contain localized anomaly sources due to isolated metal objects or small collections of objects in the trench. This is consistent with the data obtained from the soil borings which sampled metal material in some locations and not in others. The locations of these isolated anomalies are also illustrated in figure 19.

In addition to the anomalies associated with the two trenches significant isolated anomaly sources occur throughout the waste unit area. Some of these anomalies can be attributed to the presence of monitoring wells, the fence, or the concrete pads that contain steel reinforcement bars. However, other significant isolated anomalies (fig. 19) occur that can not be attributed to the presence of metal objects on the surface and probably represent buried metallic material. In particular, the isolated dipoles that occur approximately centered on grid coordinates $(42,15),(67,26)$, and $(83,26)$. 


\section{CONCLUSIONS}

The magnetic survey of the D-Area Oil Basin identified linear magnetic anomalies associated with trenches in which metallic material is buried. The southern trench exhibits lateral continuity indicating that it is either filled continuously with metallic objects or that it contains one or more long metallic objects. The northern trench exhibits isolated anomaly sources indicating that the distribution of metallic material is not continuous.

This observation would be consistent with the occurrence of single steel drums or isolated collections of a few drums.

In addition to the trenches, the waste site also exhibits isolated anomalies at other locations that probably represent buried metal objects in these locations. These anomalies are consistent with single or a few steel drums in these locations.

\section{REFERENCES}

Breiner, S. Applications Manual for Portable Magnetometers, Sunneyvale, California 94084 (1973)

Gilkeson, R. H., Gorin, S. R., Laymon, D.E. (1992) Application of magnetic and electromagnetic methods to metal detection. Proceedings of the Symposium on the Application of Geophysics to Engineering and Environmental Problems, v.1, p.309328.

Robertson, T.H. (1988) Planimetric Map of Burma Road Rubble Pit, WMO BASIN 2314F prepared for E. I. duPont deNemours and Company, Inc. by Cranston, Robertson and Whitehurst, P.C., August 31, 1988.

Sexton, B.G., Pirkle, R.J. (1992) Final Report: Ground penetrating radar investigations at the D-Area Oil Seepage Basin. A report prepared for Westinghouse Savannah River Co. by Microseeps, University of Pittsburgh Applied Research Center, Pittsburgh, PA. 
D-Area Oil Basin Magnetic Survey

Telford, W. M., Geldart, L. P., Sheriff, R. E., Keys, D. A., (1978) Applied Geophysics. Cambridge University Press, 860p. 


\section{APPENDIX: D-AREA OIL BASIN MAGNETIC SURVEY DATA}

\section{D-Area Oil Basin Diurnal Variation}

$\begin{array}{cccc}\begin{array}{c}\text { DAY 190 Diurnal Variation } \\ \text { BOTTOM }\end{array} & & & \\ \text { DAY } & \text { TIME } & \text { STA. } & \text { FIELD(nT) } \\ 190 & 9: 48: 34 & 0 & 51691.6 \\ 190 & 10: 23: 56 & 258 & 51683.8 \\ 190 & 11: 04: 16 & 556 & 51669.8 \\ 190 & 11: 48: 11 & 854 & 51664.4 \\ 190 & 13: 52: 45 & 1138 & 51679.2 \\ 190 & 14: 50: 37 & 1436 & 51699.2 \\ 190 & 15: 32: 55 & 1576 & 51712\end{array}$

$\begin{array}{cccc}\text { TOP } & & & \\ \text { DAY } & \text { TIME } & \text { STA. } & \text { FIELD (nT) } \\ 190 & 9: 48: 37 & 1 & 51694.4 \\ 190 & 10: 23: 59 & 259 & 51685.2 \\ 190 & 11: 04: 19 & 557 & 51672.4 \\ 190 & 11: 48: 15 & 855 & 51665.8 \\ 190 & 13: 52: 48 & 1139 & 51677.6 \\ 190 & 14: 50: 41 & 1437 & 51698.6 \\ 190 & 15: 32: 58 & 1577 & 51710.8\end{array}$

DAY 190 D URAL VAF ATI ON

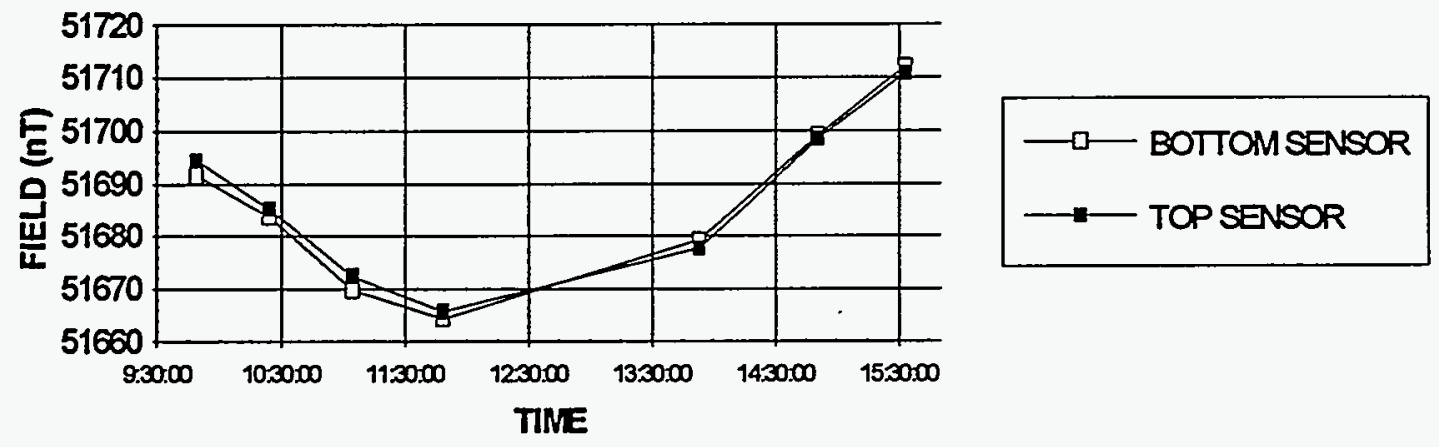

DAY 195 Diurnal Variation

$\begin{array}{cccccccc}\text { BOTTOM } & & & & & \text { TOP } \\ \text { DAY } & \text { TIME } & \text { STA. } & \text { FIELD (nT) } & \text { DAY } & \text { TIME } & \text { STA. } & \text { FIELD (nT) } \\ 195 & 9: 33: 41 & 1578 & 51699.2 & 195 & 9: 33: 44 & 1579 & 51703.0 \\ 195 & 9: 55: 56 & 1718 & 51693.8 & 195 & 9: 55: 59 & 1719 & 51697.2 \\ 195 & 10: 38: 03 & 1988 & 51689.8 & 195 & 10: 38: 06 & 1989 & 51692.8 \\ 195 & 11: 24: 40 & 2300 & 51688.0 & 195 & 11: 24: 44 & 2301 & 51691.2 \\ 195 & 13: 31: 55 & 2610 & 51679.6 & 195 & 13: 31: 58 & 2611 & 51677.6 \\ 195 & 14: 23: 48 & 2926 & 51682.0 & 195 & 14: 23: 51 & 2927 & 51683.2 \\ 195 & 15: 28: 04 & 3226 & 51699.2 & 195 & 15: 28: 07 & 3227 & 51701.6 \\ 195 & 16: 19: 53 & 3530 & 51706.6 & 195 & 16: 19: 57 & 3531 & 51709.6\end{array}$


DAY 195 D URNAL VAR ATI ON

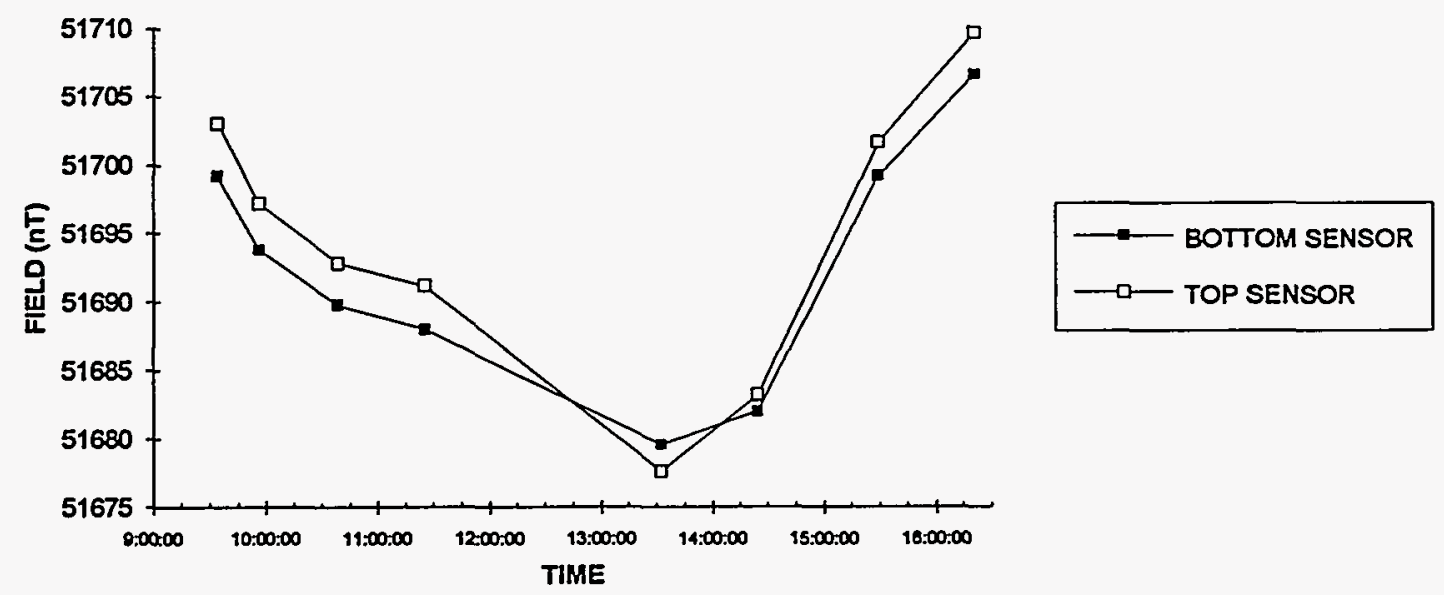

DAY 196 Diurnal Variation

\section{BOTTOM}

$\begin{array}{cccc}\text { DAY } & \text { TIME } & \text { STA. } & \text { FIELD (nT) } \\ 196 & 9: 02: 38 & 0 & 51713.0 \\ 196 & 9: 49: 15 & 318 & 51703.6 \\ 196 & 10: 32: 49 & 636 & 51698.6 \\ 196 & 13: 06: 45 & 942 & 51678.8 \\ 196 & 13: 52: 12 & 1248 & 51682.6 \\ 196 & 15: 22: 10 & 1540 & 51694.0 \\ 196 & 16: 03: 21 & 1840 & 51693.2\end{array}$

TOP

DAY TIME STA. FIELD (nT)

196

196

196

196

196

196

196
9:02:42

9:49:18

10:32:53

13:06:48

13:52:15

15:22:13

16:03:24
1

319

637

943

1249

1541

1841
51713.2

51705.2

51695.8

51680.6

51686.0

51695.4

51690.4
DAY 196 DI URNAL VARI ATION

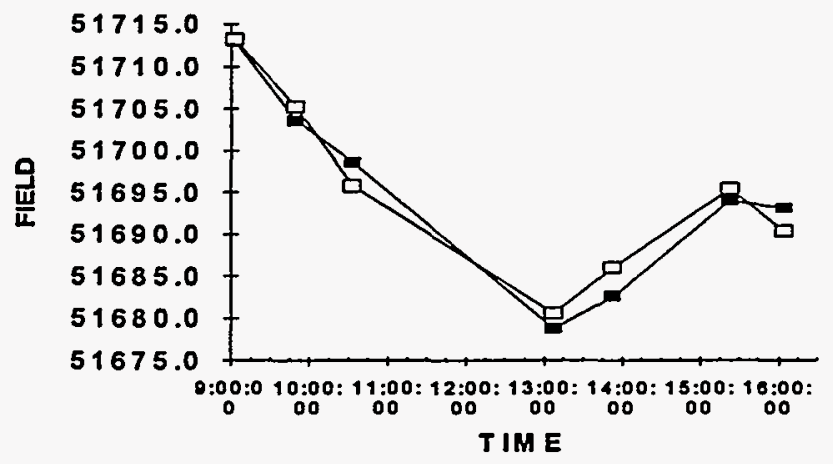

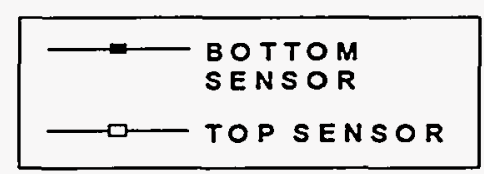

\section{DAY 203 Diurnal Variation}

BOTTOM

$\begin{array}{cccc}\text { DAY } & \text { TIME } & \text { STA. } & \text { FIELD (nT) } \\ 203 & 12: 22: 26 & 0 & 51678.5 \\ 203 & 12: 38: 52 & 32 & 51678.6 \\ 203 & 12: 46: 51 & 72 & 51678.8 \\ 203 & 12: 54: 38 & 106 & 51678.6 \\ 203 & 13: 15: 23 & 122 & 51678.0\end{array}$

$\begin{array}{cccc}\text { TOP } & & & \\ \text { DAY } & \text { TIME } & \text { STA. } & \text { FIELD (nT) } \\ 203 & 12: 22: 29 & 1 & 51678.0 \\ 203 & 12: 38: 55 & 33 & 51675.8 \\ 203 & 12: 46: 54 & 73 & 51677.0 \\ 203 & 12: 54: 42 & 107 & 51675.4 \\ 203 & 13: 15: 26 & 123 & 51675.8\end{array}$


D-Area Oil Basin Magnetic Survey

$\begin{array}{llllllll}203 & 13: 28: 39 & 190 & 51677.6 & 203 & 13: 28: 43 & 191 & 51674.2 \\ 203 & 13: 41: 53 & 244 & 51677.8 & 203 & 13: 41: 56 & 245 & 51674.4 \\ 203 & 14: 12: 56 & 352 & 51681.6 & 203 & 14: 12: 59 & 353 & 51678.2\end{array}$

Day 203 Diurnal Variation

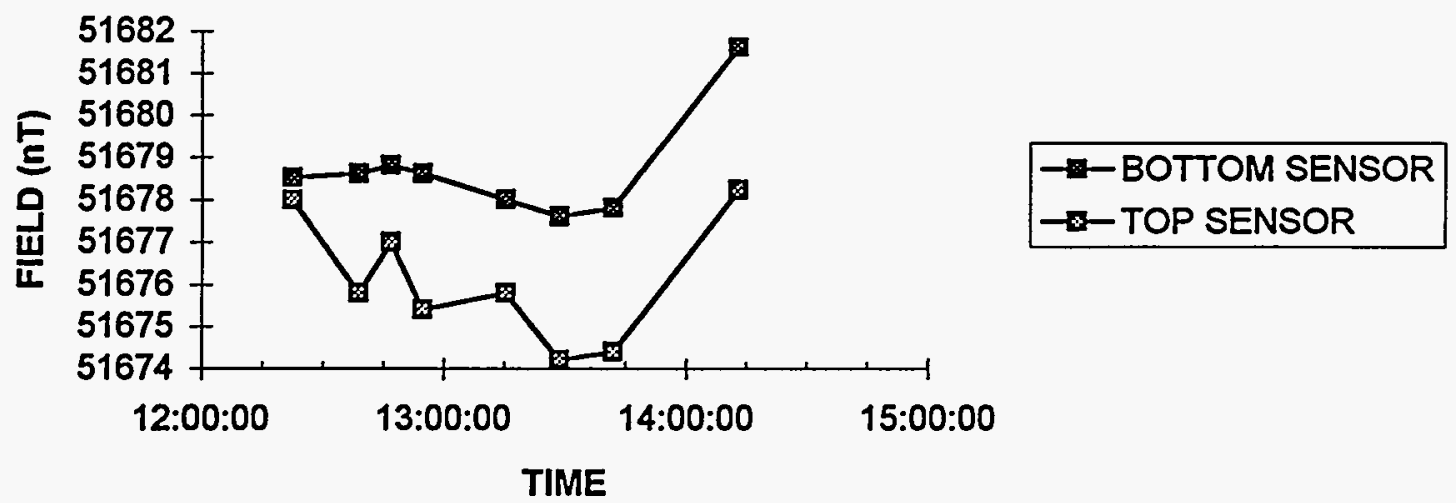

Bottom Sensor

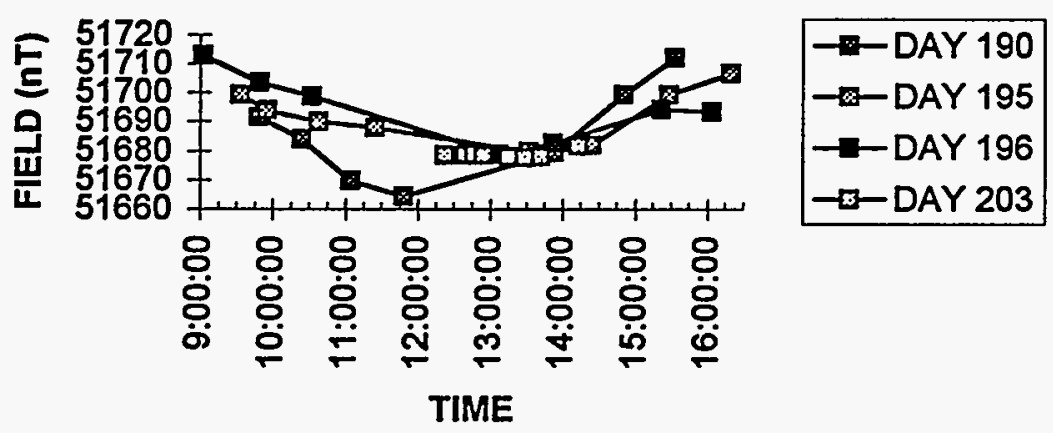




\section{TOP SENSOR}

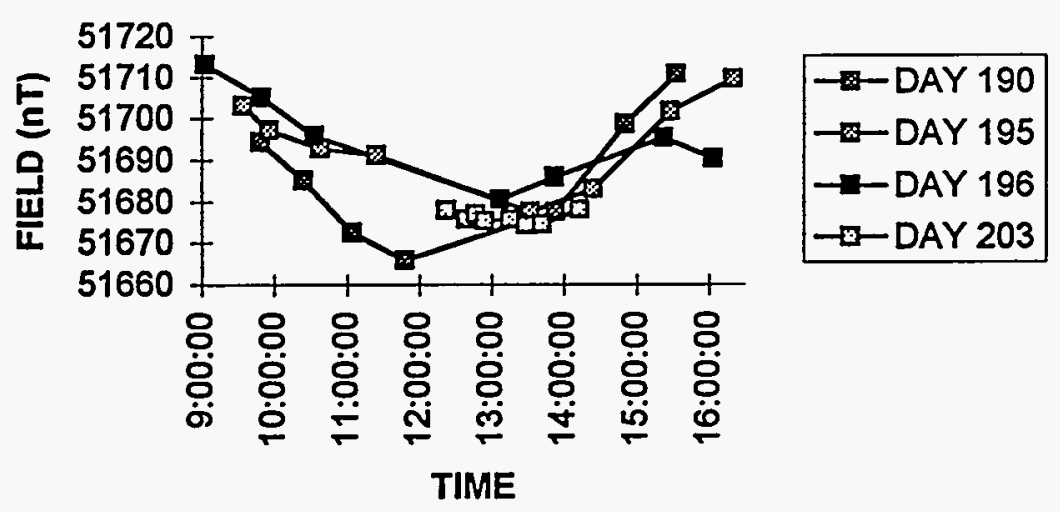


D-area Oil Basin Magnetic Survey Data

\begin{tabular}{|c|c|c|c|c|c|c|c|c|c|c|c|c|}
\hline \multirow[b]{2}{*}{ LINE } & \multirow[b]{2}{*}{ DAY } & \multirow[b]{2}{*}{ TIME } & \multirow[b]{2}{*}{ STA. } & \multirow[b]{2}{*}{$x$} & \multicolumn{3}{|c|}{ TOP FIELD (nT) } & \multicolumn{4}{|c|}{ BOTTOM FIELD (nT) } & VERTICAL \\
\hline & & & & & $\mathbf{Y}$ & (Uncorr.) & (Corr.) & (Diff.) & (Uncorr.) & (Corr.) & (Diff.) & $(\mathrm{nT} / \mathrm{m})$ \\
\hline 1 & 190 & 94920 & 2 & 0 & 0 & $? 51505.8$ & 51503.2 & 2.6 & 51330.8 & 51331.0 & -0.2 & -286.9 \\
\hline 1 & 190 & 94936 & 4 & 0 & 1 & 51554.6 & 51552.1 & 2.5 & 51472.8 & 51473.0 & -0.2 & -134.1 \\
\hline 1 & 190 & 94949 & 6 & 0 & 2 & 51612.2 & 51609.7 & 2.5 & 51575.0 & 51575.3 & -0.3 & -61.0 \\
\hline 1 & 190 & 95000 & 8 & 0 & 3 & 51645.6 & 51643.2 & 2.4 & 51622.2 & 51622.5 & -0.3 & -38.4 \\
\hline 1 & 190 & 95016 & 10 & 0 & 4 & 51667.0 & 51664.6 & 2.4 & 51656.0 & 51656.4 & -0.4 & -18.0 \\
\hline 1 & 190 & 95030 & 12 & 0 & 5 & 51677.6 & 51675.3 & 2.3 & 51665.8 & 51666.2 & -0.4 & -19.3 \\
\hline 1 & 190 & 95043 & 14 & 0 & 6 & 51684.8 & 51682.5 & 2.3 & 51671.6 & 51672.1 & -0.5 & -21.6 \\
\hline 1 & 190 & 95058 & 16 & 0 & 7 & 51689.2 & 51687 & 2.2 & 51674.8 & 51675.3 & -0.5 & -23.6 \\
\hline 1 & 190 & 95112 & 18 & 0 & 8 & 51696.2 & 51694.1 & 2.1 & 51686.2 & 51686.8 & -0.6 & -16.4 \\
\hline 1 & 190 & 95126 & 20 & 0 & 9 & 51702.8 & 51700.7 & 2.1 & 51695.0 & 51695.6 & -0.6 & -12.8 \\
\hline 1 & 190 & 95141 & 22 & 0 & 10 & 51706.4 & 51704.4 & 2.0 & 51698.2 & 51698.9 & -0.7 & -13.4 \\
\hline 1 & 190 & 95158 & $\overline{24}$ & 0 & 11 & 51710.0 & 51708.1 & 1.9 & 51706.4 & 51707.1 & -0.7 & -5.9 \\
\hline 1 & 190 & 95212 & 26 & 0 & 12 & 51712.4 & 51710.5 & 1.9 & 51707.4 & 51708.2 & -0.8 & -8.2 \\
\hline 1 & 190 & 95225 & 28 & 0 & 13 & 51713.4 & 51711.6 & 1.8 & 51709.0 & 51709.8 & -0.8 & -7.2 \\
\hline 1 & 190 & 95238 & 30 & 0 & 14 & 51715.4 & 51713.6 & 1.8 & 51711.0 & 51711.9 & -0.9 & -7.2 \\
\hline 1 & 190 & 95252 & 32 & 0 & 15 & 51716.8 & 51715.1 & 1.7 & 51712.0 & 51712.9 & -0.9 & -7.9 \\
\hline 1 & 190 & 95306 & 34 & 0 & 16 & 51717.2 & 51715.6 & 1.6 & 51710.4 & 51711.4 & -1.0 & -11.1 \\
\hline 1 & 190 & 95319 & 36 & 0 & 17 & 51718.8 & 51717.2 & 1.6 & 51715.2 & 51716.2 & -1.0 & -5.9 \\
\hline 1 & 190 & 95332 & 38 & 0 & 18 & 51719.4 & 51717.9 & 1.5 & 51715.8 & 51716.9 & -1.1 & -5.9 \\
\hline 1 & 190 & 95344 & 40 & 0 & 19 & 51721.2 & 51719.7 & 1.5 & 51719.2 & 51720.3 & -1.1 & -3.3 \\
\hline 1 & 190 & 95359 & 42 & 0 & 20 & 51722.0 & 51720.6 & 1.4 & 51719.6 & 51720.8 & -1.2 & -3.9 \\
\hline 1 & 190 & 95412 & 44 & 0 & 21 & 51722.6 & 51721.3 & 1.3 & 51720.6 & 51721.8 & -1.2 & -3.3 \\
\hline 1 & 190 & 95425 & 46 & 0 & 22 & 51723.4 & 51722.1 & 1.3 & 51721.8 & 51723.1 & -1.3 & -2.6 \\
\hline 1 & 190 & 95438 & 48 & 0 & 23 & 51724.6 & 51723.4 & 1.2 & 51721.6 & 51722.9 & -1.3 & -4.9 \\
\hline 1 & 190 & 95454 & 50 & 0 & 24 & 51725.2 & 51724 & 1.2 & 51722.0 & 51723.4 & -1.4 & -5.2 \\
\hline 1 & 190 & 95522 & 52 & 0 & 25 & 51726.6 & 51725.6 & 1.0 & 51724.6 & 51726.1 & -1.5 & -3.3 \\
\hline 1 & 190 & 95535 & 54 & 0 & 26 & 51727.6 & 51726.6 & 1.0 & 51726.2 & 51727.7 & -1.5 & -2.3 \\
\hline 1 & 190 & 95547 & 56 & 0 & 27 & 51729.8 & 51728.9 & 0.9 & 51728.2 & 51729.8 & -1.6 & -2.6 \\
\hline 1 & 190 & 95559 & 58 & 0 & 28 & 51729.8 & 51728.9 & 0.9 & 51729.8 & 51731.4 & -1.6 & 0.0 \\
\hline 1 & 190 & 95611 & 60 & 0 & 29 & 51730.6 & 51729.8 & 0.8 & 51731.2 & 51732.9 & -1.7 & 1.0 \\
\hline 1 & 190 & 95624 & 62 & 0 & 30 & 51731.8 & 51731 & 0.8 & 51733.8 & 51735.5 & -1.7 & 3.3 \\
\hline 1 & 190 & 95647 & 64 & 0 & 31 & 51732.4 & 51731.7 & 0.7 & 51735.8 & 51737.6 & -1.8 & 5.6 \\
\hline 1 & 190 & 95701 & 66 & 0 & 32 & 51732.4 & 51731.8 & 0.6 & 51738.0 & 51739.8 & -1.8 & 9.2 \\
\hline 1 & 190 & 95721 & 68 & 0 & 33 & 51737.4 & 51736.9 & 0.5 & 51735.8 & 51737.7 & -1.9 & -2.6 \\
\hline 1 & 190 & 95826 & 70 & 0 & 34 & 51733.8 & 51733.6 & 0.2 & 51737.6 & 51739.8 & -2.2 & 6.2 \\
\hline 1 & 190 & 95845 & 72 & 0 & 35 & 51729.6 & 51729.4 & 0.2 & 51739.6 & 51741.8 & -2.2 & 16.4 \\
\hline 1 & 190 & 95903 & 74 & 0 & 36 & 51725.0 & 51724.9 & 0.1 & 51736.0 & 51738.3 & -2.3 & 18.0 \\
\hline 1 & 190 & 95915 & 76 & 0 & 37 & 51715.6 & 51715.6 & 0.0 & 51729.8 & 51732.1 & -2.3 & 23.3 \\
\hline 1 & 190 & 95928 & 78 & 0 & 38 & 51705.4 & 51705.4 & 0.0 & 51721.4 & 51723.8 & -2.4 & 26.2 \\
\hline 1 & 190 & 95940 & 80 & 0 & 39 & 51692.4 & 51692.5 & -0.1 & 51709.8 & 51712.2 & -2.4 & 28.5 \\
\hline 1 & 190 & 95952 & 82 & 0 & 40 & 51677.2 & 51677.3 & -0.1 & 51696.4 & 51698.9 & -2.5 & 31.5 \\
\hline 1 & 190 & 100005 & 84 & 0 & 41 & 51662.0 & 51662.2 & -0.2 & 51684.0 & 51686.5 & -2.5 & 36.1 \\
\hline 1 & 190 & 100020 & 86 & 0 & 42 & 51650.6 & 51650.8 & -0.2 & 51667.4 & 51670.0 & -2.6 & 27.5 \\
\hline 1 & 190 & 100033 & 88 & 0 & 43 & 51641.0 & 51641.3 & -0.3 & 51656.0 & 51658.6 & -2.6 & 24.6 \\
\hline 1 & 190 & 100046 & 90 & 0 & 44 & 51634.2 & 51634.6 & -0.4 & 51639.0 & 51641.7 & -2.7 & 7.9 \\
\hline 1 & 190 & 100100 & 92 & 0 & 45 & 51633.2 & 51633.6 & -0.4 & 51639.2 & 51641.9 & -2.7 & 9.8 \\
\hline 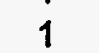 & . & . & 94 & 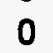 & 46 & 51633.6 & 51634.1 & -0.5 & 51637.2 & 51640.0 & -2.8 & 5.9 \\
\hline
\end{tabular}


TOP FIELD (nT)

$\begin{array}{cccccccc}\text { LINE DAY } & \text { TIME } & \text { STA. } & X & Y & \text { (Uncorr.) } & \text { (Corr.) } \\ 1 & 190 & 100124 & 96 & 0 & 47 & 51634.2 & 51634.7\end{array}$

$\begin{array}{lll}190 & 100137 & 98\end{array}$

$190 \quad 100150 \quad 100$

190100202102

$190 \quad 100214 \quad 104$

$190 \quad 100226106$

190100238108

190100250110

$190 \quad 100302112$

$190 \quad 100315 \quad 114$

$190 \quad 100334116$

$\begin{array}{lll}190 & 100346 \quad 118\end{array}$

$\begin{array}{lll}190 & 100359 & 120\end{array}$

$\begin{array}{lll}190 & 100412 \quad 122\end{array}$

$\begin{array}{lll}190 & 100428 & 124\end{array}$

$\begin{array}{lll}190 & 100517 \quad 126\end{array}$

$\begin{array}{lll}190 & 100532 & 128\end{array}$

$\begin{array}{lll}190 & 100842 \quad 130\end{array}$

$\begin{array}{lll}190 & 100858 & 132\end{array}$

$\begin{array}{lll}190 & 100911 \quad 134\end{array}$

$\begin{array}{lll}190 & 100947 & 136\end{array}$

$\begin{array}{lll}190 & 101001 & 138\end{array}$

$\begin{array}{lll}190 & 101022 & 140\end{array}$

$\begin{array}{lll}190 & 101036 & 142\end{array}$

$190101049 \quad 144$

$190 \quad 101104 \quad 146$

$190 \quad 101118 \quad 148$

$\begin{array}{lll}190 & 101142 \quad 150\end{array}$

$190 \quad 101156 \quad 152$

$\begin{array}{lll}190 & 101208 & 154\end{array}$

$\begin{array}{lll}190 & 101220 & 156\end{array}$

$\begin{array}{lll}190 & 101232 & 158\end{array}$

$\begin{array}{lll}190 & 101244 & 160\end{array}$

$\begin{array}{lll}190 & 101258 & 162\end{array}$

$190 \quad 101311 \quad 164$

$\begin{array}{lll}190 & 101324 & 166\end{array}$

$\begin{array}{lll}190 & 101337 & 168\end{array}$

$\begin{array}{lll}190 & 101353 & 170\end{array}$

$\begin{array}{lll}190 & 101406 \quad 172\end{array}$

$\begin{array}{lll}190 & 101419 & 174\end{array}$

$190 \quad 101433 \quad 176$

$\begin{array}{lll}190 & 101446 & 178\end{array}$

$\begin{array}{lll}190 & 101458 & 180\end{array}$

$\begin{array}{lll}190 & 101511 & 182\end{array}$

$\begin{array}{lll}190 & 101523 & 184\end{array}$

$\begin{array}{lll}190 & 101536 & 186\end{array}$

$\begin{array}{lll}190 & 101549 \quad 188\end{array}$

$\begin{array}{lll}190 & 101605 & 190\end{array}$

190101619192 $\begin{array}{lll}0 & 48 & 51636.6\end{array}$

$\begin{array}{lll}0 & 49 & 51638.4\end{array}$

$\begin{array}{lll}0 & 50 & 51640.8\end{array}$

$\begin{array}{lll}0 & 51 & 51644.8\end{array}$

$\begin{array}{lll}0 & 52 & 51647.4\end{array}$

$\begin{array}{lll}0 & 53 & 51651.2\end{array}$

$\begin{array}{lll}0 & 54 & 51653.2\end{array}$

$\begin{array}{lll}0 & 55 & 51656.2\end{array}$

$0 \quad 5651659.0$

$\begin{array}{lll}0 & 57 & 51663.0\end{array}$

$\begin{array}{lll}0 & 58 & 51664.8\end{array}$

$\begin{array}{lll}0 & 59 & 51665.4\end{array}$

$\begin{array}{lll}0 & 60 & 51666.4\end{array}$

$\begin{array}{lll}0 & 62 & 51661.6\end{array}$

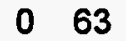

363

62

61

60

359

358

57

356

355

354

353

52

51

50

49

48

47

46

45

44

43

42

41

340

339

338

337

336

335

334

333

332
51654.2

51663.6

51666.2

51664.8

51664.2

51660.8

51655.0

51649.2

51640.8

51631.2

51622.8

51615.2

51606.2

51596.6

51588.8

51578.6

51566.8

51550.2

51534.8

51512.8

51493.2

51476.4

51486.0

51518.0

51565.0

51615.8

51665.4

51699.6

51722.4

51734.0

51738.4

51740.4

51739.0 $\begin{array}{lll}0 & 61 & 51665.4\end{array}$ \begin{tabular}{ll}
-0.5 \\
\hline
\end{tabular}

$51637.2-0.6$

$51639-0.6$

$51641.5-0.7$

$51645.5-0.7$

$51648.2-0.8$

$51652-0.8$

$51654.1-0.9$

$51657.2-1.0$

$51660-1.0$

$51664.1-1.1$

$51665.9-1.1$

$51666.6-1.2$

$51667.7-1.3$

$51666.7-1.3$

$51663.1-1.5$

$51655.8-1.6$

$\begin{array}{lll}51666 & -2.4\end{array}$

$\begin{array}{lll}51668.7 & -2.5\end{array}$

$\begin{array}{lll}51667.4 & -2.6\end{array}$

$51666.9-2.7$

$\begin{array}{lll}51663.6 & -2.8\end{array}$

$\begin{array}{lll}51657.9 & -2.9\end{array}$

$51652.1 \quad-2.9$

$51643.8-3.0$

$51634.2-3.0$

$51625.9-3.1$

$\begin{array}{lll}51618.4 & -3.2\end{array}$

$\begin{array}{lll}51609.5 & -3.3\end{array}$

$\begin{array}{lll}51599.9 & -3.3\end{array}$

$\begin{array}{lll}51592.2 & -3.4\end{array}$

$51582-3.4$

$\begin{array}{lll}51570.3 & -3.5\end{array}$

$\begin{array}{lll}51553.7 & -3.5\end{array}$

$\begin{array}{lll}51538.4 & -3.6\end{array}$

$51516.4-3.6$

$\begin{array}{lll}51496.9 & -3.7\end{array}$

$\begin{array}{lll}51480.2 & -3.8\end{array}$

$51489.8-3.8$

$\begin{array}{lll}51521.9 & -3.9\end{array}$

$\begin{array}{lll}51568.9 & -3.9\end{array}$

$51619.8-4.0$

$51669.5 \quad-4.1$

$51703.7-4.1$

$\begin{array}{lll}51726.6 & -4.2\end{array}$

$51738.2-4.2$

$51742.7-4.3$

$\begin{array}{lll}51744.7 & -4.3\end{array}$

$51743.4 \quad-4.4$
BOTTOM FIELD (nT)

GRAD.

(Uncorr.) (Corr.) (Diff.) (nT/m)

$\begin{array}{llll}51636.2 & 51639.0 & -2.8 & 3.3\end{array}$

$\begin{array}{llll}51637.0 & 51639.9 & -2.9 & 0.7\end{array}$

$\begin{array}{llll}51638.6 & 51641.5 & -2.9 & 0.3\end{array}$

$\begin{array}{llll}51640.8 & 51643.8 & -3.0 & 0.0\end{array}$

$\begin{array}{llll}51642.8 & 51645.8 & -3.0 & -3.3\end{array}$

$\begin{array}{llll}51645.0 & 51648.0 & -3.0 & -3.9\end{array}$

$\begin{array}{llll}51647.4 & 51650.5 & -3.1 & -6.2\end{array}$

$\begin{array}{llll}51650.0 & 51653.1 & -3.1 & -5.2\end{array}$

$\begin{array}{llll}51651.8 & 51655.0 & -3.2 & -7.2\end{array}$

$\begin{array}{llll}51655.0 & 51658.2 & -3.2 & -6.6\end{array}$

$\begin{array}{llll}51657.4 & 51660.7 & -3.3 & -9.2\end{array}$

$51660.0 \quad 51663.3 \quad-3.3 \quad-7.9$

$51662.4 \quad 51665.8 \quad-3.4 \quad-4.9$

$\begin{array}{llll}51660.8 & 51664.2 & -3.4 & -9.2\end{array}$

$\begin{array}{llll}51658.8 & 51662.3 & -3.5 & -10.8\end{array}$

$\begin{array}{llll}51656.6 & 51660.3 & -3.7 & -8.2\end{array}$

$51647.4 \quad 51651.1 \quad-3.7 \quad-11.1$

$51662.0 \quad 51666.4 \quad-4.4 \quad-2.6$

$\begin{array}{llll}51665.6 & 51670.1 & -4.5 & -1.0\end{array}$

$\begin{array}{llll}51665.6 & 51670.1 & -4.5 & 1.3\end{array}$

$51663.4 \quad 51668.1 \quad-4.7 \quad-1.3$

$\begin{array}{llll}51659.4 & 51664.1 & -4.7 & -2.3\end{array}$

$\begin{array}{llll}51655.2 & 51660.0 & -4.8 & 0.3\end{array}$

$\begin{array}{llll}51646.6 & 51651.4 & -4.8 & -4.3\end{array}$

$\begin{array}{llll}51638.4 & 51643.3 & -4.9 & -3.9\end{array}$

$51627.8 \quad 51632.8 \quad-5.0 \quad-5.6$

$51620.0 \quad 51625.0 \quad-5.0 \quad-4.6$

$51613.2 \quad 51618.3 \quad-5.1 \quad-3.3$

$\begin{array}{lllll}51601.4 & 51606.5 & -5.1 & -7.9\end{array}$

$\begin{array}{llll}51592.6 & 51597.8 & -5.2 & -6.6\end{array}$

$\begin{array}{llll}51589.6 & 51594.8 & -5.2 & 1.3\end{array}$

$\begin{array}{llll}51582.2 & 51587.5 & -5.3 & 5.9\end{array}$

$\begin{array}{llll}51571.8 & 51577.1 & -5.3 & 8.2\end{array}$

$\begin{array}{llll}51560.4 & 51565.8 & -5.4 & 16.7\end{array}$

$\begin{array}{llll}51547.8 & 51553.2 & -5.4 & 21.3\end{array}$

$\begin{array}{llll}51535.8 & 51541.3 & -5.5 & 37.7\end{array}$

$\begin{array}{llll}51545.6 & 51551.1 & -5.5 & 85.9\end{array}$

$\begin{array}{llll}51496.6 & 51502.2 & -5.6 & 33.1\end{array}$

$\begin{array}{llll}51535.4 & 51541.0 & -5.6 & 81.0\end{array}$

$\begin{array}{llll}51576.6 & 51582.3 & -5.7 & 96.1\end{array}$

$\begin{array}{llll}51632.4 & 51638.1 & -5.7 & 110.5\end{array}$

$\begin{array}{llll}51689.6 & 51695.4 & -5.8 & 121.0\end{array}$

$\begin{array}{llll}51735.6 & 51741.4 & -5.8 & 115.1\end{array}$

$\begin{array}{llll}51759.6 & 51765.5 & -5.9 & 98.4\end{array}$

$\begin{array}{llll}51771.6 & 51777.5 & -5.9 & 80.7\end{array}$

$\begin{array}{llll}51769.0 & 51775.0 & -6.0 & 57.4\end{array}$

$\begin{array}{lllll}51766.0 & 51772.0 & -6.0 & 45.2\end{array}$

$\begin{array}{lllll}51763.6 & 51769.7 & -6.1 & 38.0\end{array}$

$\begin{array}{llll}51756.6 & 51762.7 & -6.1 & 28.9\end{array}$ 


\section{LIN}$$
2
$$$$
2
$$$$
2
$$$$
2
$$$$
2
$$$$
2
$$$$
2
$$$$
2
$$

2

$\begin{array}{llll}3 & 190 & 103027 & 280 \\ 3 & 190 & 103040 & 282\end{array}$

$\begin{array}{lllll}3 & 190 & 103052 & 284\end{array}$

$\begin{array}{lllll}3 & 190 & 103103 & 286\end{array}$

$\begin{array}{llll}3 & 190 & 103115 & 288\end{array}$

$\begin{array}{llll}3 & 190 & 103128 & 290\end{array}$

$\begin{array}{llll}3 & 190 & 103141 & 292\end{array}$
TOP FIELD (nT)

BOTTOM FIELD (nT)

\begin{tabular}{ccccc}
$\mathbf{X}$ & $\mathbf{Y}$ & (Uncorr.) & (Corr.) & (Diff.) \\
3 & 31 & 51737.0 & 51741.5 & -4.5 \\
3 & 30 & 51734.4 & 51738.9 & -4.5 \\
3 & 29 & 51732.4 & 51737 & -4.6 \\
3 & 28 & 51731.2 & 51735.8 & -4.6 \\
3 & 27 & 51729.6 & 51734.3 & -4.7 \\
3 & 26 & 51728.2 & 51732.9 & -4.7 \\
3 & 25 & 51726.2 & 51731 & -4.8 \\
3 & 24 & 51725.2 & 51730 & -4.8 \\
3 & 23 & 51724.6 & 51729.5 & -4.9 \\
3 & 22 & 51723.8 & 51728.7 & -4.9 \\
3 & 21 & 51722.8 & 51727.8 & -5.0 \\
3 & 20 & 51721.0 & 51726 & -5.0 \\
3 & 19 & 51719.4 & 51724.5 & -5.1 \\
3 & 18 & 51719.2 & 51724.4 & -5.2 \\
3 & 17 & 51720.6 & 51725.8 & -5.2 \\
3 & 16 & 51719.2 & 51724.5 & -5.3 \\
3 & 15 & 51714.4 & 51719.8 & -5.4 \\
3 & 14 & 51711.6 & 51717 & -5.4 \\
3 & 13 & 51708.6 & 51714.1 & -5.5 \\
3 & 12 & 51708.6 & 51714.1 & -5.5 \\
3 & 11 & 51706.6 & 51712.2 & -5.6 \\
3 & 10 & 51704.4 & 51710 & -5.6 \\
3 & 9 & 51701.0 & 51706.7 & -5.7 \\
3 & 8 & 51695.4 & 51701.1 & -5.7 \\
3 & 7 & 51690.8 & 51696.6 & -5.8 \\
3 & 6 & 51683.2 & 51689.1 & -5.9 \\
3 & 5 & 51674.6 & 51680.5 & -5.9 \\
3 & 4 & 51663.8 & 51669.8 & -6.0 \\
3 & 3 & 51647.2 & 51653.2 & -6.0 \\
3 & 2 & 51625.4 & 51631.5 & -6.1 \\
3 & 1 & 51589.4 & 51595.5 & -6.1 \\
3 & 0 & 51546.0 & 51552.2 & -6.2 \\
6 & 0 & 51527.2 & 51534.3 & -7.1 \\
6 & 1 & 51606.6 & 51614.1 & -7.5 \\
6 & 2 & 51639.6 & 51647.2 & -7.6 \\
6 & 3 & 51661.6 & 51669.3 & -7.7 \\
6 & 4 & 51676.6 & 51684.3 & -7.7 \\
6 & 15 & 51716.6 & 51725.4 & -8.8 \\
6 & 5 & 51687.4 & 51695.2 & -7.8 \\
6 & 6 & 51698.6 & 51706.5 & -7.9 \\
6 & 7 & 51700.6 & 51708.8 & -8.2 \\
6 & 8 & 51704.6 & 51712.9 & -8.3 \\
6 & 9 & 51707.0 & 51715.3 & -8.3 \\
6 & 10 & 51709.0 & 51717.5 & -8.5 \\
6 & 11 & 51711.2 & 51719.7 & -8.5 \\
6 & 12 & 51713.2 & 51721.8 & -8.6 \\
\hline & 13 & 51714.4 & 51723 & -8.6 \\
\hline & & 51719.8 & 51728.6 & -8.8 \\
\hline & & & \\
6 &
\end{tabular}

BOTTOM FIELD (nT)

(Uncorr.) (Corr.) (Diff.)

$\begin{array}{lll}51750.8 & 51757.0 & -6.2\end{array}$

$51746.0 \quad 51752.2 \quad-6.2$

$51741.4 \quad 51747.6 \quad-6.2$

$51738.6 \quad 51744.9 \quad-6.3$

$51736.8 \quad 51743.1 \quad-6.3$

$51734.2 \quad 51740.6 \quad-6.4$

$51733.0 \quad 51739.4 \quad-6.4$

$\begin{array}{lll}51731.0 & 51737.5 & -6.5\end{array}$

$51728.8 \quad 51735.3 \quad-6.5$

$\begin{array}{lll}51727.0 & 51733.6 & -6.6\end{array}$

$51725.6 \quad 51732.2 \quad-6.6$

$51724.8 \quad 51731.4 \quad-6.6$

$51724.6 \quad 51731.3 \quad-6.7$

$\begin{array}{llll}51725.4 & 51732.2 & -6.8\end{array}$

$\begin{array}{llll}51727.6 & 51734.4 & -6.8\end{array}$

$\begin{array}{lll}51725.8 & 51732.7 & -6.9\end{array}$

$51715.0 \quad 51721.9 \quad-6.9$

$\begin{array}{lll}51711.0 & 51718.0 & -7.0\end{array}$

$\begin{array}{lll}51712.6 & 51719.6 & -7.0\end{array}$

$51709.8 \quad 51716.9 \quad-7.1$

$51706.8 \quad 51713.9 \quad-7.1$

$\begin{array}{llll}51703.8 & 51711.0 & -7.2\end{array}$

$\begin{array}{lll}51699.4 & 51706.6 & -7.2\end{array}$

$51694.6 \quad 51701.8 \quad-7.2$

$51689.0 \quad 51696.3 \quad-7.3$

$\begin{array}{lll}51681.6 & 51688.9 & -7.3\end{array}$

$\begin{array}{llll}51670.2 & 51677.6 & -7.4\end{array}$

$\begin{array}{lll}51655.8 & 51663.2 & -7.4\end{array}$

$51633.4 \quad 51640.9 \quad-7.5$

$\begin{array}{lll}51600.4 & 51607.9 & -7.5\end{array}$

$51536.2 \quad 51543.8 \quad-7.6$

$51413.8 \quad 51421.4 \quad-7.6$

51361.0

51548.6

$\begin{array}{lll}51640.2 & 51649.4 & -9.2 \\ 51659.6 & 51668.9 & -9.3\end{array}$

$\begin{array}{lll}51674.0 & 51683.3 & -9.3\end{array}$

$51683.6 \quad 51693.0 \quad-9.4$

$\begin{array}{lll}51690.6 & 51700.3 & -9.7\end{array}$

$\begin{array}{llll}51696.0 & 51705.8 & -9.8\end{array}$

51701.4

51702.2

51705.8

51708.2

51709.8

51710.4

51711.0

51716.2
51369.7

$-8.7$

$51557.7 \quad-9.1$

$51617.9-9.1$

GRAD.

(nT/m)

22.6

19.0

14.8

12.1

11.8

9.8

11.1

9.5

6.9

5.2

4.6

6.2

8.5

10.2

11.5

10.8

1.0

$-1.0$

6.6

2.0

0.3

$-1.0$

$-2.6$

$-1.3$

$-3.0$

$-2.6$

$-7.2$

$-13.1$

$-22.6$

$-41.0$

$-87.2$

$-216.7$

$-272.5$

$-95.1$

$-50.5$

$-35.1$

$-27.9$

$-22.0$

$-24.6$

$-16.4$

$-14.1$

$\begin{array}{lll}51711.3 & -9.9 & -9.2\end{array}$

$\begin{array}{lll}51712.2 & -10.0 & -11.1\end{array}$

$\begin{array}{lll}51715.9 & -10.1 & -8.9\end{array}$

$\begin{array}{lll}51718.4 & -10.2 & -8.2\end{array}$

$\begin{array}{lll}51720.1 & -10.3 & -7.5\end{array}$

$\begin{array}{lll}51720.7 & -10.3 & -7.5\end{array}$

$\begin{array}{lll}51721.4 & -10.4 & -9.2\end{array}$

$\begin{array}{lll}51726.7 & -10.5 & -5.9\end{array}$ 
TOP FIELD (nT)

$\begin{array}{cccccccc}\text { LINE DAY } & \text { TIME } & \text { STA. } & \mathbf{X} & \mathbf{Y} & \text { (Uncorr.) } & \text { (Corr. } \\ 3 & 190 & 103154 & 294 & 6 & 17 & 51720.4 & 51729.3\end{array}$

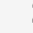

3

3

$$
\begin{array}{ll}
17 & 51720.4
\end{array}
$$

$\begin{array}{ll}19 & 51721.8\end{array}$

$\begin{array}{ll}20 & 51722.8\end{array}$

3

3

3
3
3

$\begin{array}{lllll}3 & 190 & 103154 & 294 & 6 \\ 3 & 190 & 103206 & 296 & 6 \\ 3 & 190 & 103218 & 298 & 6 \\ 3 & 190 & 103230 & 300 & 6 \\ 3 & 190 & 103243 & 302 & 6 \\ 3 & 190 & 103255 & 304 & 6 \\ 3 & 190 & 103306 & 306 & 6 \\ 3 & 190 & 103317 & 308 & 6 \\ 3 & 190 & 103329 & 310 & 6 \\ 3 & 190 & 103341 & 312 & 6 \\ 3 & 190 & 103358 & 314 & 6 \\ 3 & 190 & 103410 & 316 & 6 \\ 3 & 190 & 103422 & 318 & 6 \\ 3 & 190 & 103435 & 320 & 6 \\ 3 & 190 & 103449 & 322 & 6 \\ 3 & 190 & 103501 & 324 & 6 \\ 3 & 190 & 103514 & 326 & 6 \\ 3 & 190 & 103525 & 328 & 6 \\ 3 & 190 & 103538 & 330 & 6 \\ 3 & 190 & 103551 & 332 & 6 \\ 3 & 190 & 103603 & 334 & 6 \\ 3 & 190 & 103616 & 336 & 6 \\ 3 & 190 & 103629 & 338 & 6 \\ 3 & 190 & 103642 & 340 & 6 \\ 3 & 190 & 103656 & 342 & 6 \\ 3 & 190 & 103710 & 344 & 6 \\ 3 & 190 & 103724 & 346 & 6 \\ 3 & 190 & 103736 & 348 & 6 \\ 3 & 190 & 103750 & 350 & 6 \\ 3 & 190 & 103803 & 352 & 6 \\ 3 & 190 & 103814 & 354 & 6 \\ 3 & 190 & 103826 & 356 & 6 \\ 3 & 190 & 103839 & 358 & 6 \\ 3 & 190 & 103851 & 360 & 6 \\ 3 & 190 & 103904 & 362 & 6 \\ 3 & 190 & 103916 & 364 & 6 \\ 3 & 190 & 103928 & 366 & 6 \\ 3 & 190 & 103941 & 368 & 6 \\ 3 & 190 & 103953 & 370 & 6 \\ 3 & 190 & 104006 & 372 & 6 \\ 3 & 190 & 104018 & 374 & 6 \\ 3 & 190 & 104029 & 376 & 6 \\ 3 & 190 & 104040 & 378 & 6 \\ 3 & 190 & 104050 & 380 & 6 \\ 3 & 190 & 104103 & 382 & 6 \\ 3 & 190 & 104117 & 384 & 6 \\ 3 & 190 & 104129 & 386 & 6 \\ 3 & 190 & 104142 & 388 & 6 \\ 3 & 190 & 104157 & 390 & 6 \\ & & & & \end{array}$

BOTTOM FIELD (nT)

GRAD.

$\begin{array}{ll}18 & 51720.4\end{array}$

$\begin{array}{lll}6 & 21 & 51723.4\end{array}$

$\begin{array}{lll}6 & 22 & 51723.8\end{array}$

$\begin{array}{lll}6 & 23 & 51724.2\end{array}$

$\begin{array}{lll}6 & 24 & 51725.2\end{array}$

$\begin{array}{lll}25 & 51725.6\end{array}$

$\begin{array}{lll}26 & 51726.0\end{array}$

$\begin{array}{ll}27 & 51727.0\end{array}$

$\begin{array}{ll}28 & 51728.2\end{array}$

$\begin{array}{ll}29 & 51729.2\end{array}$

$\begin{array}{ll}30 & 51731.4\end{array}$

$\begin{array}{lll}31 & 51733.2\end{array}$

3251734.6

$\begin{array}{ll}33 & 51734.4\end{array}$

$\begin{array}{ll}34 & 51732.2\end{array}$

$\begin{array}{ll}35 & 51720.8\end{array}$

36

37

51680.4

51578.8

$\begin{array}{ll}38 & 51367.4\end{array}$

$\begin{array}{ll}39 & ? 50979.8\end{array}$

$\begin{array}{ll}40 & ? 50621.4\end{array}$

$\begin{array}{ll}41 & ? 50500.8\end{array}$

$42 ? 50702.2$

$\begin{array}{ll}43 & 50924.0\end{array}$

$\begin{array}{ll}44 & 51130.2\end{array}$

$\begin{array}{ll}45 & 51270.6\end{array}$

$\begin{array}{ll}46 & 51372.4\end{array}$

$\begin{array}{ll}47 & 51449.4\end{array}$

$\begin{array}{ll}48 & 51518.8\end{array}$

$\begin{array}{ll}49 & 51587.4\end{array}$

$\begin{array}{ll}50 & 51573.0\end{array}$

5151550.8

$52 \quad 51549.4$

$\begin{array}{ll}53 & 51548.4\end{array}$

$\begin{array}{ll}54 & 51574.6\end{array}$

$\begin{array}{ll}55 & 51606.4\end{array}$

$\begin{array}{ll}56 & 51624.4\end{array}$

$\begin{array}{ll}57 & 51638.0\end{array}$

$\begin{array}{ll}58 & 51650.0\end{array}$

$\begin{array}{ll}59 & 51655.4\end{array}$

$\begin{array}{ll}60 & 51660.2\end{array}$

$\begin{array}{ll}61 & 51662.8\end{array}$

$\begin{array}{ll}62 & 51664.0\end{array}$

$\begin{array}{ll}63 & 51664.0\end{array}$

$\begin{array}{ll}64 & 51660.4\end{array}$

$\begin{array}{ll}65 & 51650.4\end{array}$
- 3.9

$51729.4-9.0$

$51730.8-9.0$

$51731.9-9.1$

$51732.6-9.2$

$\begin{array}{ll}51733 & -9.2\end{array}$

$51733.5-9.3$

$51734.6-9.4$

$51735 \quad-9.4$

$\begin{array}{lll}51735.5 & -9.5\end{array}$

$51736.6-9.6$

$51737.8-9.6$

$51738.9-9.7$

$51741.2-9.8$

$\begin{array}{lll}51743 & -9.8\end{array}$

$51744.5-9.9$

$51744.4-10.0$

$51742.2-10.0$

$51730.9-10.1$

$51690.6-10.2$

$\begin{array}{lll}51589 & -10.2\end{array}$

$51377.7-10.3$

$50990.2-10.4$

$50631.8-10.4$

$50511.3-10.5$

$50712.8-10.6$

$\begin{array}{lll}50934.7 & -10.7\end{array}$

$\begin{array}{ll}51140.9 & -10.7\end{array}$

$\begin{array}{ll}51281.4 & -10.8\end{array}$

$51383.3-10.9$

$\begin{array}{lll}51460.3 & -10.9\end{array}$

$51529.8-11.0$

$51598.5-11.1$

$51584.1-11.1$

$51562-11.2$

$51560.7-11.3$

$51559.7-11.3$

$51586 \quad-11.4$

$51617.8-11.4$

$51635.9-11.5$

$51649.6-11.6$

$51661.6-11.6$

$51667.1-11.7$

$51671.9-11.7$

$51674.6-11.8$

$51675.9-11.9$

$51676-12.0$

$51672.4-12.0$

$51662.5-12.1$
(Uncorr)

51717.4

51717.6

51718.6

51717.8

51721.4

51722.0

51722.6

51724.6

51725.4

51726.8

51728.8

51731.0

51735.0

51739.4

51744.8

51753.0

51763.8

51778.0

51791.6

51795.2

?51756.4

?51594.2

?51164.6

?50590.8

?50410

?50663

50916.4

51139.0

51265.8

51355.6

51422.4

51466.2

?51674.8

51562.0

51535.6

51570.2

51501.2

51528.2

51573.0

51594.0

51611.4

51636.2

51640.4

51646.4

51652.4

51655.8

51658.2

51658.0

51653.8
(Corr.) (Diff.)

$51727.9-10.5$

$51728.2-10.6$

$51729.3-10.7$

$51728.6-10.8$

$51732.2-10.8$

$51732.9-10.9$

$51733.6-11.0$

$51735.6-11.0$

$51736.5-11.1$

$51738.0-11.2$

$51740.1-11.3$

$51742.3-11.3$

$51746.4-11.4$

$51750.9-11.5$

$51756.4-11.6$

$51764.6-11.6$

$51775.5 \quad-11.7$

$51789.8-11.8$

$51803.4-11.8$

$51807.1-11.9$

$51768.4-12.0$

$51606.3-12.1$

$51176.7-12.1$

$50603.0-12.2$

$50422.3-12.3$

$50675.4 \quad-12.4$

$50928.9-12.5$

$51151.5 \quad-12.5$

$51278.4 \quad-12.6$

$51368.3 \quad-12.7$

$51435.1-12.7$

$51479.0-12.8$

$51687.7-12.9$

$\begin{array}{ll}51575.0 & -13.0\end{array}$

$51548.6-13.0$

$51583.3-13.1$

$51514.4-13.2$

$\begin{array}{lll}51541.4 & -13.2\end{array}$

$51586.3-13.3$

$51607.4-13.4$

$51624.9-13.5$

$51649.7-13.5$

$51654.0-13.6$

$51660.0-13.6$

$51666.1-13.7$

$51669.6-13.8$

$51672.1-13.9$

$51671.9-13.9$

$51667.8-14.0$
(nT/m)

$-4.9$

$-4.6$

$-5.2$

$-8.2$

$-3.3$

$-3.0$

$-2.6$

$-1.0$

$-0.3$

1.3

3.0

4.6

9.5

13.1

19.0

30.2

48.2

75.1

116.1

188.2

291.2

371.8

303.0

$-50.2$

$-148.9$

$-64.3$

$-12.5$

14.4

$-7.9$

$-27.5$

$-44.3$

$-86.2$

143.3

$-18.0$

$-24.9$

34.1

$-77.4$

$-76.1$

$-54.8$

$-49.8$

$-43.6$

$-22.6$

$-24.6$

$-22.6$

$-17.0$

$-13.4$

$-9.5$

$-3.9$

5.6 
TOP FIELD (nT)

LINE DAY TIME STA$$
4 \quad 190 \quad 104828 \quad 424
$$$$
4 \quad 190 \quad 104840 \quad 426
$$$$
4 \quad 190 \quad 104851 \quad 428
$$$$
4 \quad 190 \quad 104902 \quad 430
$$$$
4 \quad 190 \quad 104913 \quad 432
$$$$
4 \quad 190 \quad 104930 \quad 434
$$$$
4 \quad 190 \quad 104942 \quad 436
$$$$
4 \quad 190 \quad 104956 \quad 438
$$$$
4 \quad 190 \quad 105010 \quad 440
$$$$
4 \quad 190 \quad 105022 \quad 442
$$$$
4 \quad 190 \quad 105035 \quad 444
$$$$
4 \quad 190 \quad 105050 \quad 446
$$$$
\begin{array}{llll}
4 & 190 & 105103 & 448
\end{array}
$$$$
4 \quad 190 \quad 105122 \quad 450
$$$$
4 \quad 190 \quad 105202 \quad 452
$$$$
4 \quad 190 \quad 105216 \quad 454
$$$$
4 \quad 190 \quad 105229 \quad 456
$$$$
4 \quad 190 \quad 105241 \quad 458
$$$$
4190105254 \quad 460
$$$$
4 \quad 190 \quad 105309 \quad 462
$$$$
4 \quad 190 \quad 105322 \quad 464
$$$$
\begin{array}{llll}
4 & 190 & 105337 & 466
\end{array}
$$$$
4 \quad 190 \quad 105351 \quad 468
$$$$
4190 \quad 105406 \quad 470
$$$$
\begin{array}{llll}
4 & 190 & 105421 & 472
\end{array}
$$$$
4 \quad 190 \quad 105436 \quad 474
$$$$
4 \quad 190 \quad 105450 \quad 476
$$$$
4 \quad 190 \quad 105504 \quad 478
$$$$
4 \quad 190 \quad 105517 \quad 480
$$$$
\begin{array}{llll}
4 & 190 & 105529 & 482
\end{array}
$$$$
4 \quad 190 \quad 105542 \quad 484
$$$$
4 \quad 190 \quad 105557 \quad 486
$$

$4 \quad 190 \quad 105612 \quad 488$

\begin{tabular}{|c|c|c|c|c|c|c|c|c|}
\hline & \multicolumn{3}{|c|}{ TOP FIELD (nT) } & \multicolumn{4}{|c|}{ BOTTOM FIELD (nT) } & \multirow{2}{*}{$\begin{array}{l}\text { GRAD. } \\
\text { (nT/m) }\end{array}$} \\
\hline & $\mathbf{Y}$ & (Uncorr.) & (Corr.) & (Diff.) & (Uncorr.) & (Corr.) & (Diff.) & \\
\hline & 66 & & 51638.6 & -12.2 & 51637.6 & & & 8.4 \\
\hline & 67 & & & -12.2 & & & -14.2 & 9.0 \\
\hline & 68 & & & -12.3 & & & & \\
\hline & 69 & 37.6 & & -12.4 & & & & 4.4 \\
\hline & 70 & 53.8 & & -12.4 & & & & -68.5 \\
\hline & 71 & 51527.0 & 51539.5 & -12.5 & 04.4 & .9 & -14.5 & -37.0 \\
\hline & 72 & 34.6 & 97.2 & -12.6 & 69.2 & .7 & -14.5 & -25.3 \\
\hline & 73 & 22.8 & 35.6 & -12.8 & 13.8 & .5 & -14.7 & -14.8 \\
\hline & 73 & 3.0 & 36.6 & -13.6 & 36.6 & & -15.7 & \\
\hline & 72 & 9.2 & 32.9 & -13.7 & 5.0 & & -1 & -138.0 \\
\hline & 71 & .4 & .1 & -13.7 & 4.8 & & & 0.3 \\
\hline & 70 & .8 & & -13.8 & .6 & 5 & -15.9 & 117.7 \\
\hline & 69 & .6 & & -13.9 & 77.4 & & -16.0 & 33.6 \\
\hline & 68 & & 2.4 & -14.0 & 9.8 & & -16.1 & 33.4 \\
\hline & 67 & 0.4 & 0.4 & -14.0 & 3.2 & & -16.1 & 52.1 \\
\hline & 66 & 2.6 & 6.7 & -14.1 & & & -16.2 & 24.9 \\
\hline & 65 & 8.8 & & -14.2 & & 516 & -16.3 & 12.8 \\
\hline & 64 & 1.8 & 66 & -14.2 & & .2 & -16.4 & $\begin{array}{l}4.9 \\
\text { - }\end{array}$ \\
\hline & 63 & 516 & 72.3 & -14.3 & .0 & 516 & -16.4 & 1.6 \\
\hline & 62 & 51 & 6.7 & -14.3 & 6 & 1 & -16.5 & -9.5 \\
\hline & 61 & 51 & 9.4 & -14.4 & & & -16.6 & -18.7 \\
\hline & 60 & 51 & 2.1 & -14.5 & & & -16.7 & -28.2 \\
\hline & 59 & & & -14.6 & & & -16.7 & .9 \\
\hline & 58 & & & -14.6 & & & -16.8 & -61.0 \\
\hline & 57 & .2 & & -14.7 & & & -16.9 & -76.7 \\
\hline & 56 & 0.2 & & -14.8 & & & -17.0 & -11.5 \\
\hline & 55 & 2.0 & & -14.8 & 7.6 & & -17.0 & 304.3 \\
\hline & 54 & 518 & 4.7 & -14.9 & & 520 & -17.1 & 351.1 \\
\hline & 53 & 6.0 & 1 & -15.0 & 4.6 & 516 & -17.2 & 79.7 \\
\hline & 52 & 6.8 & & -15.1 & 9.8 & 51 & -17.3 & -454.1 \\
\hline & 51 & 13 & 8.3 & -15.3 & 26.2 & 50 & -17.5 & -798.0 \\
\hline & 50 & & & -15.4 & & & -17.6 & -151.5 \\
\hline & 49 & & & 15.4 & & & -17.7 & \\
\hline & 48 & & & 5 & & & .8 & .3 \\
\hline & 47 & & & & & & -17.8 & 77.4 \\
\hline & 46 & & 0.5 & -15.7 & & & -17.9 & 229.8 \\
\hline & 45 & 2.4 & 8.1 & -15.7 & 78.8 & 509 & -18.0 & -251.8 \\
\hline & 44 & 0.6 & 50716.4 & -15.8 & 93.2 & 504 & -18.1 & -503.9 \\
\hline & 43 & $? 50202.2$ & 50218.1 & -15.9 & 34.8 & 498 & -18.2 & -684.3 \\
\hline & 42 & ?49399 & & -16.0 & & 48 & -18.3 & \\
\hline & 41 & 5.6 & & -16.0 & 8.6 & 46 & -18.3 & 4.9 \\
\hline & 40 & $? 52$ & & -16.1 & & & & 10602.0 \\
\hline & 39 & $? 50$ & 2.4 & -16.2 & 81.6 & 499 & -18.5 & \\
\hline & 38 & 509 & & -16.3 & 75.2 & & & 302.3 \\
\hline & 37 & & & -16.3 & 52.2 & & -18.7 & 263.9 \\
\hline & 36 & 51 & 77.6 & -16.4 & 2.6 & & -18.7 & 182.6 \\
\hline & 35 & 51637.2 & 51653.7 & -16.5 & 51704.4 & 51723.2 & -18.8 & 110.2 \\
\hline & 34 & 51674.6 & 51691.1 & -16.5 & & 51737.5 & -18.9 & 72. \\
\hline & 33 & 51688.8 & 51705.4 & -16.6 & 51718.6 & 51737.6 & -19.0 & 48.9 \\
\hline
\end{tabular}


TOP FIELD (nT)

LINE DAY TIME STA. $X \quad Y$ (Uncorr.) (Corr.)

西

4

4

5

5

5

5

5
5

$$
\begin{array}{lllll}
190 & 105628 & 490 & 9 & 32
\end{array}
$$$$
\begin{array}{lllll}
190 & 105640 & 492 & 9 & 31
\end{array}
$$$$
\begin{array}{lllll}
190 & 105651 & 494 & 9 & 30
\end{array}
$$$$
190 \quad 105703 \quad 496
$$$$
190 \quad 105716 \quad 498
$$$$
190 \quad 105727 \quad 500
$$$$
190105738 \quad 502
$$$$
190 \quad 105750 \quad 504
$$$$
190105801506
$$$$
190 \quad 105814 \quad 508
$$$$
\begin{array}{lll}
190 & 105832 \quad 510
\end{array}
$$$$
\begin{array}{lll}
190 & 105844 & 512
\end{array}
$$$$
\begin{array}{lll}
190 & 105857 & 514
\end{array}
$$$$
190 \quad 105910 \quad 516
$$$$
\begin{array}{lll}
190 & 105924 & 518
\end{array}
$$$$
\begin{array}{lll}
190 & 105941 \quad 520
\end{array}
$$$$
190 \quad 105953 \quad 522
$$$$
190 \quad 110009 \quad 524
$$$$
\begin{array}{lll}
190 & 110022 & 526
\end{array}
$$$$
\begin{array}{lll}
190 & 110036 \quad 528
\end{array}
$$$$
190110048 \quad 530
$$$$
\begin{array}{lll}
190 & 110102 \quad 532
\end{array}
$$

$31 \quad 51698.8$

$30 \quad 51702.6$

$29 \quad 51704.2$

2851705.0

2751706.6

2651706.6

$25 \quad 51707.4$

2451707.6

2351706.6

$22 \quad 51707.4$

2151707.4

$\begin{array}{lll}9 & 20 & 51706.4\end{array}$

$\begin{array}{lll}9 & 19 & 51707.0\end{array}$

$\begin{array}{lll}9 & 18 & 51705.8\end{array}$

$\begin{array}{lll}9 & 17 & 51705.6\end{array}$

$9 \quad 1651704.2$

$9 \quad 15 \quad 51702.0$

$\begin{array}{lll}9 & 14 & 51702.0\end{array}$

$\begin{array}{lll}9 & 13 & 51700.2\end{array}$

$\begin{array}{lll}9 & 12 & 51699.2\end{array}$

$\begin{array}{lll}9 & 11 & 51696.8\end{array}$

$9 \quad 10 \quad 51696.2$

$\begin{array}{lll}9 & 9 & 51693.4\end{array}$

98

97

96

95

94

93

92

91

90

120

121

122

123

124

125

126

127

128

129

1210

1211

1212

1213

1214

1215
(Corr.) (Diff.) (Uncorr.) $51711.3-16.7$

$51715.6-16.8$

$51719.4-16.8$

$51721.1-16.9$

$51722-17.0$

$51723.6-17.0$

$51723.7-17.1$

$51724.5-17.1$

$51724.8-17.2$

$51723.9-17.3$

$51724.8-17.4$

$51724.8-17.4$

$51723.9-17.5$

$51724.6-17.6$

$51723.4-17.6$

$51723.3-17.7$

$51722-17.8$

$51719.9-17.9$

$51719.9-17.9$

$51718.2-18.0$

$51717.3-18.1$

$51715-18.2$

$51714.4-18.2$

$51711.7-18.3$

$51710.8-18.4$

$51712.6-18.4$

$51718.9-18.5$

$51724.2-18.6$

$51714.6-18.6$

$51680.9-18.7$

$51648.6-18.8$

$51606.8-18.8$

$51550.8-19.0$

$51548.8-19.6$

$51618.9-19.9$

$51658.5-19.9$

$51668-20.0$

$51627.6-20.0$

$51621.5-20.1$

$51671.7-20.1$

$51694.7-20.1$

$51705.6-20.2$

$51711.2-20.2$

$51714.8-20.2$

$51717.5-20.3$

$51719.5-20.3$

$51720.5-20.3$

$51722.6-20.4$

$51723.4-20.4$ 51718.0

51718.0

51716.0

51715.6

51714.8

51714.6

51714.2

51714.2

51713.4

51712.4

51711.6

51696.0

51694.2

51691.8

51691.4

51695.0

51695.8

51670.6

51628.0

51590.0

51518.2

51354.4

51391.8

51546.0

51614.0

51644.2

51619.8

51662.0

51677.6

51684.8

51688.2

51696.4

51697.6
BOTTOM FIELD (nT) (Corr.) (Diff.)

51711.6

51710.0

51709.8

51708.0

51708.0

51706.0

51703.8

51703.4

51701.0

51698.6

?51049.8

$? 51345.2$

51691.4

51694.4

51698.6

$51727.3-19.1$

$51737.1-19.1$

$51737.2-19.2$

$51735.3-19.3$

$51734.9-19.3$

$51734.2-19.4$

$51734.1-19.5$

$51733.7-19.5$

$51733.8-19.6$

$51733.1-19.7$

$51732.2-19.8$

$51731.5-19.9$

$51731.5 \quad-19.9$

$51730.0 \quad-20.0$

$51729.9-20.1$

$51728.2-20.2$

$51728.3-20.3$

$51726.4-20.4$

$51724.2-20.4$

$51723.9-20.5$

$51721.6-20.6$

$51719.3-20.7$

$51716.7-20.7$ 
TOP FIELD (nT)

BOTTOM FIELD (nT)

GRAD.

LINE DAY TIME STA. $X \quad Y$ (Uncorr.) (Corr.) (Diff.) (Uncorr.) (Corr.) (Diff.) (nT/m)

\begin{tabular}{|c|c|c|c|c|c|c|c|c|c|c|c|}
\hline 90 & & 590 & 12 & 16 & 51703.8 & 724.2 & -20.4 & 01100 & 51723.4 & -22.8 & -5.2 \\
\hline 90 & 11242 & 592 & 12 & 17 & 51703.4 & 51723.9 & -20.5 & 51701.0 & 51723.8 & -22.8 & -3.9 \\
\hline 190 & 11255 & 594 & 12 & 18 & 1703.8 & 724.3 & -20.5 & & 1723.9 & -22.9 & -4.6 \\
\hline 190 & 111307 & 596 & 12 & 19 & 51703.6 & 724.1 & -20.5 & 51700.6 & 1723.5 & -22.9 & -4.9 \\
\hline 190 & 111320 & 598 & 12 & 20 & 51703.4 & 1724 & -20.6 & 51701.0 & 723.9 & -22.9 & -3.9 \\
\hline 190 & 111332 & 600 & & 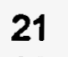 & & 724 & -20.6 & & & -22.9 & -4.6 \\
\hline 190 & & 602 & & 2 & 2.6 & 23.2 & -20.6 & & & -23.0 & 39 \\
\hline 190 & 1356 & 604 & & 20 & & 1721.8 & -20.6 & & 22.8 & -23.0 & -2.3 \\
\hline 90 & 1407 & 606 & 12 & 24 & 99.8 & 51720.5 & -20.7 & 9.0 & 722.0 & -23.0 & -1.3 \\
\hline 190 & 1419 & 608 & 12 & 25 & 98.4 & 51719.1 & -20.7 & 8.2 & 21.2 & -23.0 & -0.3 \\
\hline 190 & 1437 & 610 & 12 & 26 & 96.2 & 51716.9 & -20.7 & 5.6 & 18.7 & -23.1 & -1.0 \\
\hline 190 & 1449 & 612 & 12 & 27 & 93.0 & 51713.8 & -20.8 & & 16.5 & -23.1 & 0.7 \\
\hline 190 & & 614 & & 28 & 39.8 & 0.6 & -20.8 & & 12.9 & -23.1 & 0.0 \\
\hline 190 & & 616 & & 29 & 33.8 & & -20.8 & & & -23.1 & 1.0 \\
\hline 90 & & 618 & & 30 & 7.0 & 516 & -20.9 & & & -23.2 & 2.3 \\
\hline 190 & 39 & 620 & & 31 & 9.4 & 0.3 & -20.9 & .0 & 92.2 & -23.2 & -0.7 \\
\hline 90 & & 622 & & 3 & 0.8 & 7.7 & -20.9 & & 0.2 & -23.2 & 0.3 \\
\hline 90 & 34 & 624 & 12 & Ju & 4.2 & 5.2 & -21.0 & & 7.8 & -23.2 & 0.7 \\
\hline 90 & 17 & 626 & 12 & 34 & 2.4 & 3.4 & -21.0 & .4 & 18.7 & -23.3 & 11.5 \\
\hline 90 & 329 & 628 & 12 & 35 & 6.0 & 87 & -21.0 & 1.4 & 3.7 & -23.3 & 58.4 \\
\hline 90 & 1643 & 630 & 12 & 36 & 4.6 & 5.7 & -21.1 & & 0.7 & -23.3 & 208.5 \\
\hline 90 & 57 & 632 & 12 & 37 & 39.0 & 0.1 & -21.1 & & 68.8 & -23.4 & \\
\hline 90 & 1712 & 634 & 12 & 38 & 34.0 & 5.1 & -21.1 & & 28.4 & -23.4 & 0.3 \\
\hline 90 & 11727 & 636 & 12 & 39 & 358 & 9.2 & -21.2 & .4 & 2.8 & -23.4 & 95.7 \\
\hline 30 & 1743 & 638 & 12 & 40 & $? 52006$ & 520 & -21.2 & .2 & 3.6 & -23.4 & 41.3 \\
\hline 190 & 1804 & 640 & 12 & 41 & ?52068.4 & 52089.7 & -21.3 & .4 & 38.9 & -23.5 & 5.4 \\
\hline 90 & 327 & 642 & 12 & 42 & 978 & 9.3 & -21.3 & .6 & 4.1 & -23.5 & 12.5 \\
\hline 190 & 43 & 644 & 12 & 43 & 4.2 & 5.6 & -21.4 & & 4.4 & -23.6 & 07.5 \\
\hline 190 & 57 & 646 & 12 & 44 & 9.8 & 1.2 & -21.4 & .2 & 9.8 & -23.6 & -71.5 \\
\hline 190 & 16 & 648 & 12 & 45 & 3.2 & 4.6 & -21.4 & & 7.6 & -23.6 & -195.4 \\
\hline 190 & 28 & 650 & 12 & 46 & 7.0 & & -21.5 & & .5 & -23.7 & 3.3 \\
\hline 90 & & 652 & 12 & 47 & & & -21.5 & & 5.5 & -23.7 & -40.0 \\
\hline 90 & & 654 & 12 & 48 & 3.8 & 51645.4 & -21.6 & & 4.1 & -23.7 & -120.3 \\
\hline 90 & 112011 & 656 & 12 & 49 & 78.4 & 51600 & -21.6 & 9.8 & 53.6 & -23.8 & -79.7 \\
\hline 90 & 112026 & 658 & 12 & 50 & 51467.0 & 51488.6 & -21.6 & .8 & 30.6 & -23.8 & 147.2 \\
\hline 90 & 112043 & 660 & 12 & 51 & 55.2 & 51276.9 & -21.7 & .4 & 37.2 & -23.8 & -462.0 \\
\hline 190 & 12057 & 662 & 12 & 52 & 14.8 & 6.5 & -21.7 & 5.4 & 9.2 & -23.8 & 919.0 \\
\hline 19 & 11 & 664 & 12 & 53 & 0.2 & 7.9 & -21.7 & 2.6 & 5 & -23.9 & 5.4 \\
\hline . & & 666 & 12 & 54 & 9.4 & 1.2 & -21.8 & & 2.3 & -23.9 & 49.2 \\
\hline 190 & & 668 & 12 & 55 & 26.6 & 525 & -21.8 & & 2.9 & -23.9 & 08.9 \\
\hline an & 12155 & 670 & 12 & 56 & 23.4 & 52 & -21.8 & & 2.2 & -24.0 & 89.8 \\
\hline 90 & 12209 & 672 & 12 & 57 & 1.8 & 52 & -21.9 & 7.8 & 1.8 & -24.0 & -170.5 \\
\hline 90 & 12222 & 674 & 12 & 58 & 51831.6 & 51853.5 & -21.9 & 31.2 & 55.2 & -24.0 & -164.6 \\
\hline 190 & 12233 & 676 & 12 & 59 & 51742.4 & 51764.3 & -21.9 & 76.0 & 700.0 & -24.0 & -108.9 \\
\hline 190 & 12249 & 678 & 12 & 60 & 51703.8 & 51725.8 & -22.0 & 51657.4 & 81.5 & -24.1 & -76.1 \\
\hline 190 & 12303 & 680 & 12 & 61 & 51681.8 & 51703.8 & -22.0 & 51651.6 & 75.7 & -24.1 & -49.5 \\
\hline 190 & 12317 & 682 & 12 & 62 & 51670.4 & 51692.4 & -22.0 & & 1.5 & -24.1 & -37.7 \\
\hline 19 & & 684 & 12 & 63 & & & -22.1 & & 9.6 & -24.2 & -24.9 \\
\hline 10 & 342 & 686 & 12 & 64 & 51650.4 & 51672.5 & -22.1 & & 84.2 & -24.2 & -17.1 \\
\hline
\end{tabular}


TOP FIELD (nT)

LINE DAY TIME STA.

\begin{tabular}{|c|c|c|c|c|c|c|c|c|}
\hline & 90 & & 688 & 12 & 65 & 51633.4 & 51655.5 & -22 \\
\hline & 190 & 112406 & 690 & 12 & 66 & 51605.2 & 51627.4 & -22.2 \\
\hline & 190 & 112418 & 692 & 12 & 07 & & 566 & 2 \\
\hline & 90 & 31 & 94 & 12 & 8 & & 27.6 & -22.2 \\
\hline & 190 & 149 & 96 & 12 & & & & -22 \\
\hline & 190 & 112502 & 98 & 2 & 70 & & & -22 \\
\hline & 190 & 112517 & 700 & 12 & 1 & & 06.3 & -22.3 \\
\hline & 190 & 112530 & 702 & 12 & ? & & 15.2 & -22 \\
\hline & 190 & 112544 & 704 & 12 & 3 & 46.4 & 568.8 & -22.4 \\
\hline & 190 & 112930 & 706 & 15 & 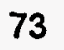 & 0.6 & 3.6 & -23.0 \\
\hline & 190 & 112943 & 708 & 15 & 72 & & & -23.0 \\
\hline & 90 & 113000 & 710 & 5 & & 25.4 & 8.5 & -23.1 \\
\hline & 190 & 113 & 712 & 15 & & 9.6 & 2.7 & -23.1 \\
\hline & 190 & 113 & 714 & 5 & & & & -23.2 \\
\hline & 19 & 113 & 716 & & 8 & & & -23.2 \\
\hline & 190 & 113 & 718 & 15 & 7 & & & -23.2 \\
\hline & 190 & 113 & 720 & 15 & 6 & & 1.9 & -23.3 \\
\hline & 190 & 113 & 722 & 15 & 5 & & 5.5 & -23.3 \\
\hline & 190 & 113 & 724 & 15 & 4 & & 0.5 & -23.3 \\
\hline & 190 & 113 & 726 & 15 & 3 & & 3.2 & -23.4 \\
\hline & 190 & 113213 & 728 & 15 & 2 & & 17.2 & -23.4 \\
\hline & 190 & 1132 & 730 & 15 & 1 & & & -23 \\
\hline & 190 & 113242 & 732 & 15 & & .0 & 57.5 & -23.5 \\
\hline & 190 & 113 & 73 & 15 & & & 7.7 & -23.5 \\
\hline & 190 & 113 & 73 & $1 !$ & & & 6.1 & -23.5 \\
\hline & 190 & 113 & 73 & & & & 5.2 & -23.6 \\
\hline & 190 & 113 & 740 & & & & & -23.6 \\
\hline & 190 & 11 & 742 & & & & .4 & -23.6 \\
\hline & 190 & 11 & 744 & 15 & 4 & & .5 & -23.7 \\
\hline & 190 & 113 & 746 & 15 & 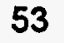 & & & -23.7 \\
\hline & 190 & 113 & 748 & 15 & 2 & & 0.9 & -23.7 \\
\hline & 190 & 113 & 750 & 1 & 1 & & 0.4 & -23.8 \\
\hline & 190 & 1135 & 752 & 15 & 50 & & 3.1 & -23.9 \\
\hline & 190 & 113 & 754 & 15 & 9 & & 4.5 & -23.9 \\
\hline & 190 & 113549 & 756 & 1 & 8 & & 5.5 & -23.9 \\
\hline & 190 & 113 & 758 & 15 & 7 & & 9.4 & -24.0 \\
\hline & 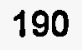 & & 76 & & & & & -24.0 \\
\hline & 190 & & 762 & & 5 & & 0.3 & -24.1 \\
\hline & 190 & & 76 & & 4 & & & -24.1 \\
\hline & 190 & & 766 & & 3 & & & -24.2 \\
\hline 6 & 190 & 113 & 768 & 1. & 2 & & 5.8 & -24.2 \\
\hline 5 & 190 & 113 & 770 & 15 & 1 & & 3.2 & -24.2 \\
\hline 6 & 190 & 113823 & 772 & 15 & 40 & 0.2 & 04.5 & -24.3 \\
\hline 6 & 190 & 113838 & 774 & 15 & 39 & & 1.6 & -24.4 \\
\hline & 190 & 113 & 776 & 15 & 38 & & & -24.4 \\
\hline & 190 & 113 & 778 & . & 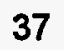 & & 32.2 & -24 \\
\hline 5 & 19 & & 780 & 1 & 36 & & & -24.5 \\
\hline 6 & 19 & 113 & 782 & 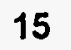 & 3 & & & -24 . \\
\hline & 90 & 113947 & 784 & & & 1.8 & 51576.3 & 0 \\
\hline
\end{tabular}

BOTTOM FIELD (nT)

GRAD.

(Uncorr.) (Corr.) (Diff.) (nT/m)

$\begin{array}{llll}51629.0 & 51653.2 & -24.2 & -7.2\end{array}$

$\begin{array}{llll}51609.2 & 51633.4 & -24.2 & 6.6\end{array}$

$\begin{array}{llll}51565.2 & 51589.5 & -24.3 & 35.1\end{array}$

$\begin{array}{llll}51466.6 & 51490.9 & -24.3 & 100.3\end{array}$

$\begin{array}{lllll}251247.6 & 51271.9 & -24.3 & 286.2\end{array}$

$\begin{array}{llll}? 50587.2 & 50611.5 & -24.3 & 276.7\end{array}$

$\begin{array}{lllll}250715.6 & 50740.0 & -24.4 & -440.0\end{array}$

$\begin{array}{lllll}51305.2 & 51329.6 & -24.4 & -143.6\end{array}$

$\begin{array}{lllll}51504.4 & 51528.8 & -24.4 & -68.9\end{array}$

$\begin{array}{lllll}51517.2 & 51542.1 & -24.9 & -87.5\end{array}$

$\begin{array}{llll}51297.0 & 51321.9 & -24.9 & -211.5\end{array}$

$\begin{array}{lllll}250661.8 & 50686.8 & -25.0 & -268.2\end{array}$

$\begin{array}{lllll}? 50612 & 50637.0 & -25.0 & -242.0\end{array}$

$\begin{array}{lllll}251303.6 & 51328.6 & -25.0 & 110.2\end{array}$

$\begin{array}{lllll}51527.4 & 51552.5 & -25.1 & 97.4\end{array}$

$\begin{array}{llll}51618.4 & 51643.5 & -25.1 & 68.2\end{array}$

$51630.2 \quad 51655.3 \quad-25.1 \quad 19.0$

$\begin{array}{llll}51642.8 & 51668.0 & -25.2 & 1.0\end{array}$

$51648.0 \quad 51673.2 \quad-25.2 \quad-15.1$

$\begin{array}{llll}51656.0 & 51681.2 & -25.2 & -22.6\end{array}$

$\begin{array}{llll}51662.8 & 51688.0 & -25.2 & -34.4\end{array}$

$\begin{array}{llll}51671.8 & 51697.1 & -25.3 & -56.1\end{array}$

$\begin{array}{llll}51692.8 & 51718.1 & -25.3 & -83.9\end{array}$

$\begin{array}{lllll}51733.0 & 51758.3 & -25.3 & -133.1\end{array}$

$\begin{array}{lllll}51840.4 & 51865.7 & -25.3 & -183.9\end{array}$

$\begin{array}{lllll}? 52105.6 & 52131.0 & -25.4 & -173.8\end{array}$

$\begin{array}{llll}352713.2 & 52738.6 & -25.4 & 176.7\end{array}$

$\begin{array}{llll}353566.6 & 53592.0 & -25.4 & 1180.0\end{array}$

$\begin{array}{lllll}253409.6 & 53435.0 & -25.4 & 1193.1\end{array}$

$\begin{array}{llll}351928.4 & 51953.9 & -25.5 & -93.1\end{array}$

$\begin{array}{lllll}250963.8 & 50989.3 & -25.5 & -726.9\end{array}$

$\begin{array}{llll}? 50906.4 & 50932.0 & -25.6 & -574.1\end{array}$

51395.8

?51728.2

?51137.6

51496.0

51859.6

52209.0

$? 52729.2$

$? 53120.4$

$? 53550.2$

?53441.4

$? 52809.2$

52169.6

?52133.2

$? 52024.4$

?51213

51404.4

51421.

$-25.6$

2.6

$51753.8-25.6$

$\begin{array}{ll}51163.3 & -25.7\end{array}$

$\begin{array}{ll}51521.7 & -25.7\end{array}$

$51885.3 \quad-25.7$

$\begin{array}{ll}52234.8 & -25.8\end{array}$

$52755.0-25.8$

$53146.3 \quad-25.9$

$53576.1-25.9$

$53467.3-25.9$

$52835.2-26.0$

$52195.6-26.0$

$52159.2-26.0$

$52050.5-26.1$

$51239.1-26.1$

$51430.5-26.1$

$51551.8-26.2$

323.9

$-596.7$

$-146.6$

86.6

266.9

615.4

767.2

1161.6

1118.7

539.3

$-12.5$

153.4

51525.6

256.7

$-596.7$

$-171.5$

$-43.0$ 
TOP FIELD (nT)

BOTTOM FIELD (nT)

GRAD.

LINE DAY TIME STA. $X \quad Y$ (Uncorr.) (Corr.) (Diff.) (Uncorr.) (Corr.) (Diff.) (nT/m)

$\begin{array}{lllllllllllll}6 & 190 & 114000 & 786 & 15 & 33 & 51590.2 & 51614.8 & -24.6 & 51581.8 & 51608.0 & -26.2 & -13.8\end{array}$

$\begin{array}{lllllllllllll}6 & 190 & 114013 & 788 & 15 & 32 & 51615.6 & 51640.2 & -24.6 & 51608.8 & 51635.0 & -26.2 & -11.1\end{array}$

$\begin{array}{lllllllllllll}6 & 190 & 114024 & 790 & 15 & 31 & 51636.0 & 51660.6 & -24.6 & 51639.6 & 51665.8 & -26.2 & 5.9\end{array}$

$\begin{array}{lllllllllllll}6 & 190 & 114035 & 792 & 15 & 30 & 51652.2 & 51676.8 & -24.6 & 51659.6 & 51685.9 & -26.3 & 12.1\end{array}$

$\begin{array}{lllllllllllll}6 & 190 & 114052 & 794 & 15 & 29 & 51663.0 & 51687.7 & -24.7 & 51671.2 & 51697.5 & -26.3 & 13.4\end{array}$

$\begin{array}{llllllllllllll}6 & 190 & 114105 & 796 & 15 & 28 & 51671.0 & 51695.7 & -24.7 & 51677.6 & 51703.9 & -26.3 & 10.8\end{array}$

$\begin{array}{lllllllllllll}6 & 190 & 114116 & 798 & 15 & 27 & 51676.4 & 51701.2 & -24.8 & 51683.4 & 51709.7 & -26.3 & 11.5\end{array}$

$\begin{array}{lllllllllllll}6 & 190 & 114129 & 800 & 15 & 26 & 51681.6 & 51706.4 & -24.8 & 51687.2 & 51713.6 & -26.4 & 9.2\end{array}$

$\begin{array}{lllllllllllll}6 & 190 & 114142 & 802 & 15 & 25 & 51685.2 & 51710 & -24.8 & 51691.4 & 51717.8 & -26.4 & 10.2\end{array}$

$\begin{array}{lllllllllllll}6 & 190 & 114153 & 804 & 15 & 24 & 51689.0 & 51713.8 & -24.8 & 51696.6 & 51723.0 & -26.4 & 12.5\end{array}$

$\begin{array}{lllllllllllll}6 & 190 & 114206 & 806 & 15 & 23 & 51689.8 & 51714.7 & -24.9 & 51692.4 & 51718.8 & -26.4 & 4.3\end{array}$

$\begin{array}{lllllllllllll}6 & 190 & 114219 & 808 & 15 & 22 & 51690.2 & 51715.1 & -24.9 & 51692.0 & 51718.5 & -26.5 & 3.0\end{array}$

$\begin{array}{lllllllllllll}6 & 190 & 114232 & 810 & 15 & 21 & 51691.6 & 51716.5 & -24.9 & 51695.0 & 51721.5 & -26.5 & 5.6\end{array}$

$\begin{array}{lllllllllllll}6 & 190 & 114247 & 812 & 15 & 20 & 51692.0 & 51717 & -25.0 & 51697.0 & 51723.5 & -26.5 & 8.2\end{array}$

$\begin{array}{lllllllllllll}6 & 190 & 114258 & 814 & 15 & 19 & 51693.0 & 51718 & -25.0 & 51697.6 & 51724.2 & -26.6 & 7.5\end{array}$

$\begin{array}{lllllllll}6 & 190 & 114311 & 816 & 15 & 18 & 51694.6 & 51719.6 & -25.0\end{array}$

$\begin{array}{llllllllll}6 & 190 & 114326 & 818 & 15 & 17 & 51695.0 & 51720.1 & -25.1\end{array}$

$\begin{array}{llllllllll}6 & 190 & 114338 & 820 & 15 & 16 & 51695.2 & 51720.3 & -25.1\end{array}$

$\begin{array}{llllllllll}6 & 190 & 114351 & 822 & 15 & 15 & 51694.4 & 51719.5 & -25.1\end{array}$

$\begin{array}{llllllllll}6 & 190 & 114404 & 824 & 15 & 14 & 51693.4 & 51718.6 & -25.2\end{array}$

$\begin{array}{lllllllll}6 & 190 & 114418 & 826 & 15 & 13 & 51692.6 & 51717.8 & -25.2\end{array}$

$\begin{array}{llllllllll}6 & 190 & 114430 & 828 & 15 & 12 & 51692.2 & 51717.4 & -25.2\end{array}$

$\begin{array}{llllllllll}6 & 190 & 114442 & 830 & 15 & 11 & 51690.0 & 51715.3 & -25.3\end{array}$

$\begin{array}{lllllllll}6 & 190 & 114454 & 832 & 15 & 10 & 51687.2 & 51712.5 & -25.3\end{array}$

$\begin{array}{llllllllll}6 & 190 & 114506 & 834 & 15 & 9 & 51683.4 & 51708.7 & -25.3\end{array}$

$\begin{array}{lllllllll}6 & 190 & 114518 & 836 & 15 & 8 & 51679.4 & 51704.8 & -25.4\end{array}$

$\begin{array}{lllllllll}6 & 190 & 114531 & 838 & 15 & 7 & 51672.8 & 51698.2 & -25.4\end{array}$

$\begin{array}{llllllllll}6 & 190 & 114543 & 840 & 15 & 6 & 51663.2 & 51688.6 & -25.4\end{array}$

$\begin{array}{llllllllll}6 & 190 & 114555 & 842 & 15 & 5 & 51652.0 & 51677.4 & -25.4\end{array}$

$\begin{array}{lllllllll}6 & 190 & 114607 & 844 & 15 & 4 & 51646.6 & 51672.1 & -25.5\end{array}$

$\begin{array}{lllllllll}6 & 190 & 114619 & 846 & 15 & 3 & 51641.2 & 51666.7 & -25.5\end{array}$

$\begin{array}{lllllllll}6 & 190 & 114636 & 848 & 15 & 2 & 51628.6 & 51654.2 & -25.6\end{array}$

$\begin{array}{lllllllll}6 & 190 & 114648 & 850 & 15 & 1 & 51597.4 & 51623 & -25.6\end{array}$

$\begin{array}{lllllllll}6 & 190 & 114704 & 852 & 15 & 0 & 51531.6 & 51557.2 & -25.6\end{array}$

$\begin{array}{llll}7 & 190 & 131257 & 856\end{array}$

$\begin{array}{lllll}7 & 190 & 131314 & 858\end{array}$

$\begin{array}{lll}18 & 0 & 51550.8\end{array}$

$51557.2-25.6$

51698.0

$51724.6-26.6$

5.6

$51698.6 \quad 51725.2 \quad-26.6 \quad 5.9$

$51699.0 \quad 51725.6 \quad-26.6 \quad 6.2$

$\begin{array}{llll}51698.2 & 51724.9 & -26.7 & 6.2\end{array}$

$\begin{array}{lllll}51696.6 & 51723.3 & -26.7 & 5.2\end{array}$

$\begin{array}{llll}51695.2 & 51721.9 & -26.7 & 4.3\end{array}$

$\begin{array}{llll}51694.2 & 51720.9 & -26.7 & 3.3\end{array}$

$\begin{array}{lllll}51691.6 & 51718.4 & -26.8 & 2.6\end{array}$

$\begin{array}{llll}7 & 190 & 131328 & 860\end{array}$

$\begin{array}{lll}18 & 1 & 51614.8\end{array}$

$51632.5-17.7$

51688.0

51714.8

51683.8

51710.6

$-26.8$

1.3

51678.0

51704.8

51670.2

51697.

51659.6

51686.5

51648.8

51675.7

51641.8

51668.7

51632.6

51659.6

51611.6

51638.6

51563.8

51590.8

51452.4

51479.5

$-26.8$

0.7

51404.4

51421.5

$-26.8$

$-2.3$

$\begin{array}{llll}7 & 190 & 131341 & 862\end{array}$

$\begin{array}{lll}18 & 2 & 51655.2\end{array}$

$51672.9-17.7$

51544.0

51561.1

$-26.9$

$-4.3$

$\begin{array}{lllll}7 & 190 & 131355 & 864\end{array}$

$\begin{array}{lll}18 & 3 & 51675.8\end{array}$

$51693.5-17.7$

51608.2

51625.3

$-26.9$

$-5.9$

$7 \quad 190 \quad 131410 \quad 866$

$\begin{array}{lll}18 & 4 & 51687.6\end{array}$

$51705.3-17.7$

51639.4

51656.4

$-26.9$

$-5.2$

$\begin{array}{llll}7 & 190 & 131424 & 868\end{array}$

$\begin{array}{lll}18 & 5 & 51689.8\end{array}$

$51707.5-17.7$

51658.0

51675.0

$-26.9$

$-7.9$

$7 \quad 190 \quad 131436 \quad 870$

$\begin{array}{lll}18 & 6 & 51695.2\end{array}$

$51712.8-17.6$

51670.4

51687.4

$-27.0$

$-14.1$

$\begin{array}{llll}7 & 190 & 131451 & 872\end{array}$

$\begin{array}{lll}18 & 7 & 51699.8\end{array}$

$51717.4-17.6$

51678.6

51695.6

$-27.0$

$-27.9$

$\begin{array}{lllll}7 & 190 & 131504 & 874\end{array}$

$\begin{array}{lll}18 & 8 & 51703.6\end{array}$

$51721.2-17.6$

51684.2

51701.1

$-27.0$

$-55.1$

$\begin{array}{lllll}7 & 190 & 131519 & 876\end{array}$

$\begin{array}{lll}18 & 9 & 51706.4\end{array}$

$51724-17.6$

51689.4

51706.3

$-27.1$

$-129.8$

$\begin{array}{llll}7 & 190 & 131532 & 878\end{array}$

$\begin{array}{lll}18 & 10 & 51708.0\end{array}$

$51725.6-17.6$

51693.6

51710.5

$-17.1$

$-240.0$

$\begin{array}{llll}7 & 190 & 131545 & 880\end{array}$

$\begin{array}{lll}18 & 11 & 51709.0\end{array}$

$51726.5-17.5$

51696.4

51713.3

$-17.1$

$-116.1$

$\begin{array}{lll}18 & 12 & 51709.2\end{array}$

$51726.7-17.5$

51699.4

51716.2

$-17.1$

$-77.0$

$\begin{array}{llll}7 & 190 & 131558 & 882\end{array}$

$\begin{array}{lll}18 & 13 & 51709.4\end{array}$

$51726.9-17.5$

51703.8

51718.

$-17.0$

$-59.7$

$\begin{array}{llll}7 & 190 & 131611 & 884\end{array}$

$51728.7-17.5$

51703.2

51720.0

$-17.0$

$-48.5$

$-31.8$

$-27.2$

$-25.6$

$-23.3$

$-21.0$

$-19.0$

$-15.7$

$-12.1$

$-9.2$

$-13.1$ 
TOP FIELD (nT)

\section{LIN}

7

$\begin{array}{llll}7 & 190 & 131625 & 886\end{array}$

$\begin{array}{llll}7 & 190 & 131637 & 888\end{array}$

$\begin{array}{llll}7 & 190 & 131652 & 890\end{array}$

$\begin{array}{lllll}7 & 190 & 131706 & 892\end{array}$

$\begin{array}{llll}7 & 190 & 131719 & 894\end{array}$

$7 \quad 190 \quad 131735 \quad 896$

$\begin{array}{llll}7 & 190 & 131752 & 898\end{array}$

$\begin{array}{llll}7 & 190 & 131806 & 900\end{array}$

$\begin{array}{llll}7 & 190 & 131820 & 902\end{array}$

$\begin{array}{llll}7 & 190 & 131834 & 904\end{array}$

$7 \quad 190 \quad 131846 \quad 906$

$\begin{array}{lllll}7 & 190 & 131858 & 908\end{array}$

$7 \quad 190 \quad 131910 \quad 910$

$\begin{array}{llll}7 & 190 & 131923 & 912\end{array}$

$\begin{array}{llll}7 & 190 & 131935 & 914\end{array}$

$\begin{array}{llll}7 & 190 & 131948 & 916\end{array}$

$\begin{array}{llll}7 & 190 & 132001 & 918\end{array}$

$\begin{array}{llll}7 & 190 & 132015 & 920\end{array}$

$\begin{array}{llll}7 & 190 & 132028 & 922\end{array}$

$\begin{array}{llll}7 & 190 & 132043 & 924\end{array}$

$\begin{array}{lllll}7 & 190 & 132057 & 926\end{array}$

$\begin{array}{llll}7 & 190 & 132113 & 928\end{array}$

$\begin{array}{llll}7 & 190 & 132127 & 930\end{array}$

$\begin{array}{llll}7 & 190 & 132143 & 932\end{array}$

$\begin{array}{llll}7 & 190 & 132157 & 934\end{array}$

$\begin{array}{lllll}7 & 190 & 132214 & 936\end{array}$

$\begin{array}{llll}7 & 190 & 132230 & 938\end{array}$

$7 \quad 190 \quad 132245 \quad 940$

$\begin{array}{llll}7 & 190 & 132302 & 942\end{array}$

$\begin{array}{llll}7 & 190 & 132315 & 944\end{array}$

$\begin{array}{llll}7 & 190 & 132330 & 946\end{array}$

$\begin{array}{llll}7 & 190 & 132344 & 948\end{array}$

$\begin{array}{llll}7 & 190 & 132355 & 950\end{array}$

$\begin{array}{llll}7 & 190 & 132410 & 952\end{array}$

$\begin{array}{llll}7 & 190 & 132423 & 954\end{array}$

$\begin{array}{llll}7 & 190 & 132439 & 956\end{array}$

$\begin{array}{llll}7 & 190 & 132454 & 958\end{array}$

$\begin{array}{llll}7 & 190 & 132510 & 960\end{array}$

$\begin{array}{llll}7 & 190 & 132528 & 962\end{array}$

$\begin{array}{llll}7 & 190 & 132543 & 964\end{array}$

$\begin{array}{llll}7 & 190 & 132559 & 966\end{array}$

$\begin{array}{llll}7 & 190 & 132612 & 968\end{array}$

$\begin{array}{llll}7 & 190 & 132625 & 970\end{array}$

$\begin{array}{llll}7 & 190 & 132639 & 972\end{array}$

$\begin{array}{llll}7 & 190 & 132654 & 974\end{array}$

$\begin{array}{llll}7 & 190 & 132708 & 976\end{array}$

$\begin{array}{llll}7 & 190 & 132722 & 978\end{array}$

$\begin{array}{llll}7 & 190 & 132740 & 980\end{array}$

$\begin{array}{llll}7 & 190 & 132753 & 982\end{array}$
(Uncorr.) (Corr.)

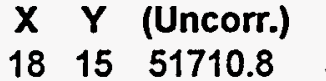

$\begin{array}{lll}18 & 16 & 51710.8\end{array}$

$\begin{array}{lll}18 & 17 & 51709.2\end{array}$

$\begin{array}{lll}18 & 18 & 51709.4\end{array}$

$\begin{array}{lll}18 & 19 & 51708.4\end{array}$

$\begin{array}{lll}18 & 20 & 51708.0\end{array}$

$\begin{array}{lll}18 & 21 & 51705.8\end{array}$

$\begin{array}{lll}18 & 22 & 51703.8\end{array}$

$\begin{array}{lll}18 & 23 & 51701.0\end{array}$

$\begin{array}{lll}18 & 24 & 51698.8\end{array}$

$\begin{array}{lll}18 & 25 & 51696.2\end{array}$

$\begin{array}{lll}18 & 26 & 51692.6\end{array}$

$\begin{array}{lll}18 & 27 & 51688.2\end{array}$

$\begin{array}{lll}18 & 28 & 51683.6\end{array}$

$\begin{array}{lll}18 & 29 & 51674.8\end{array}$

$\begin{array}{lll}18 & 30 & 51665.4\end{array}$

$\begin{array}{lll}18 & 31 & 51651.0\end{array}$

$\begin{array}{lll}18 & 32 & 51634.0\end{array}$

$18 \quad 33$

1834

1835

$18 \quad 36$

$18 \quad 37$

$18 \quad 38$

$18 \quad 39$

1840

$\begin{array}{lll}18 & 41\end{array}$

$\begin{array}{lll}18 & 42\end{array}$

1843

$\begin{array}{ll}18 & 44\end{array}$

\begin{tabular}{ll}
$18 \quad 45$ \\
\hline
\end{tabular}

1846

$\begin{array}{lll}18 & 47\end{array}$

$\begin{array}{lll}18 & 48\end{array}$

$18 \quad 49$

?51118.2

$\begin{array}{lll}18 & 51 & ? 51060.2\end{array}$

$\begin{array}{lll}18 & 52 & ? 51100.8\end{array}$

$\begin{array}{lll}18 & 53 & ? 51467\end{array}$

$\begin{array}{lll}18 & 54 & ? 52375\end{array}$

$\begin{array}{lll}18 & 55 & ? 53285.4\end{array}$

$\begin{array}{lll}18 & 56 & ? 53335\end{array}$

$\begin{array}{lll}18 & 57 & ? 52822.4\end{array}$

1858

52268.0

$\begin{array}{lll}18 & 59 & 51973.4\end{array}$

$\begin{array}{lll}18 & 60 & 51842.4\end{array}$

$\begin{array}{lll}18 & 61 & 51783.8\end{array}$

$\begin{array}{lll}18 & 62 & 51746.6\end{array}$

1863 $\begin{array}{lll}51728.2 & -17.4 & 517 \\ 51728.2 & -17.4 & 517 \\ 51726.6 & -17.4 & 517 \\ 51726.8 & -17.4 & 517\end{array}$

$51725.8 \quad-17.4$

$51725.3-17.3$

$51723.1-17.3$

$51721.1-17.3$

$51718.3-17.3$

$\begin{array}{lll}51716 & -17.2\end{array}$

$51713.4-17.2$

$51709.8-17.2$

$51705.4 \quad-17.2$

$51700.8-17.2$

$51691.9-17.1$

$51682.5-17.1$

$51668.1-17.1$

$51651.1-17.1$

$51625.1-17.1$

$51591.2-17.0$

$51559.4-17.0$

$51599.6-17.0$

$51757.8-17.0$

$51840.9-16.9$

$51833.9-16.9$

$51865.5-16.9$

$51912.3-16.9$

$51949.6-16.8$

$51966.8-16.8$

$51919.4-16.8$

$51830-16.8$

$51765.4-16.8$

$51647.5-16.7$

$51483.7-16.7$

$51296.3-16.7$

$51134.9-16.7$

$51076.8-16.6$

$51117.4-16.6$

$51483.6-16.6$

$52391.6-16.6$

$53301.9-16.5$

$53351.5-16.5$

$52838.9-16.5$

$52284.5-16.5$

$51989.9-16.5$

$51858.8-16.4$

$51800.2-16.4$

$51763-16.4$

$51739.8-16.4$
BOTTOM FIELD (nT)

GRAD.

(Uncorr.) (Corr.) (Diff.) (nT/m)

$\begin{array}{lllll}51703.8 & 51720.5 & -16.7 & -11.5\end{array}$

$\begin{array}{llll}51704.0 & 51720.7 & -16.7 & -11.1\end{array}$

$\begin{array}{llll}51704.0 & 51720.7 & -16.7 & -8.5\end{array}$

$51702.4 \quad 51719.0 \quad-16.6 \quad-11.5$

$\begin{array}{llll}51701.8 & 51718.4 & -16.6 & -10.8\end{array}$

$\begin{array}{lllll}51700.4 & 51717.0 & -16.6 & -12.5\end{array}$

$\begin{array}{llll}51701.0 & 51717.6 & -16.6 & -7.9\end{array}$

$51699.0 \quad 51715.5 \quad-16.5 \quad-7.9$

$\begin{array}{llll}51699.8 & 51716.3 & -16.5 & -2.0\end{array}$

$\begin{array}{lllll}51696.2 & 51712.7 & -16.5 & -4.3\end{array}$

$\begin{array}{llll}51692.4 & 51708.8 & -16.4 & -6.2\end{array}$

$\begin{array}{llll}51688.6 & 51705.0 & -16.4 & -6.6\end{array}$

$\begin{array}{llll}51681.0 & 51697.4 & -16.4 & -11.8\end{array}$

$\begin{array}{llll}51676.8 & 51693.2 & -16.4 & -11.1\end{array}$

$\begin{array}{llll}51671.4 & 51687.7 & -16.3 & -5.6\end{array}$

$\begin{array}{llll}51660.6 & 51676.9 & -16.3 & -7.9\end{array}$

$\begin{array}{llll}51646.6 & 51662.9 & -16.3 & -7.2\end{array}$

$\begin{array}{lllll}51625.6 & 51641.9 & -16.3 & -13.8\end{array}$

$\begin{array}{llll}51594.2 & 51610.4 & -16.2 & -22.6\end{array}$

$\begin{array}{llll}51540.2 & 51556.4 & -16.2 & -55.7\end{array}$

$\begin{array}{lllll}51452.6 & 51468.8 & -16.2 & -147.2\end{array}$

$\begin{array}{lllll}? 51396.4 & 51412.6 & -16.2 & -305.2\end{array}$

$\begin{array}{lllll}? 51836.2 & 51852.3 & -16.1 & 156.4\end{array}$

$\begin{array}{lllll}251772.2 & 51788.3 & -16.1 & -84.9\end{array}$

$\begin{array}{lllll}51702.4 & 51718.5 & -16.1 & -187.9\end{array}$

$\begin{array}{llll}51827.0 & 51843.0 & -16.0 & -35.4\end{array}$

$\begin{array}{lllll}51846.8 & 51862.8 & -16.0 & -79.7\end{array}$

$\begin{array}{llll}51913.6 & 51929.6 & -16.0 & -31.5\end{array}$

$\begin{array}{llll}51968.4 & 51984.3 & -15.9 & 30.2\end{array}$

$\begin{array}{llll}51921.2 & 51937.1 & -15.9 & 30.5\end{array}$

$\begin{array}{llll}51785.4 & 51801.3 & -15.9 & -45.6\end{array}$

$\begin{array}{llll}51765.2 & 51781.1 & -15.9 & 27.2\end{array}$

$\begin{array}{llll}51633.2 & 51649.0 & -15.8 & 3.9\end{array}$

$\begin{array}{llll}51417.6 & 51433.4 & -15.8 & -81.0\end{array}$

$\begin{array}{lllll}51150.8 & 51166.6 & -15.8 & -211.1\end{array}$

$\begin{array}{lllll}550756.2 & 50771.9 & -15.7 & -593.4\end{array}$

$\begin{array}{lllll}50847.6 & 50863.3 & -15.7 & -348.5\end{array}$

$\begin{array}{lllll}250713 & 50728.7 & -15.7 & -635.7\end{array}$

$\begin{array}{lllll}\text { ?50773.6 } & 50789.2 & -15.6 & -1136.7\end{array}$

$\begin{array}{lllll}252694 & 52709.6 & -15.6 & 523.0\end{array}$

$\begin{array}{lllll}? 54627.4 & 54643.0 & -15.6 & 2200.0\end{array}$

$\begin{array}{lllll}? 54481.8 & 54497.4 & -15.6 & 1880.0\end{array}$

$\begin{array}{lllll}? 53247.6 & 53263.1 & -15.5 & 697.0\end{array}$

$\begin{array}{lllll}? 52161.2 & 52176.7 & -15.5 & -175.1\end{array}$

$\begin{array}{llll}51848.0 & 51863.5 & -15.5 & -205.6\end{array}$

$\begin{array}{lllll}51752.8 & 51768.2 & -15.4 & -146.9\end{array}$

$\begin{array}{lllll}51717.2 & 51732.6 & -15.4 & -109.2\end{array}$

$\begin{array}{llll}51696.4 & 51711.8 & -15.4 & -82.3\end{array}$

$\begin{array}{llll}51685.4 & 51700.8 & -15.4 & -62.3\end{array}$ 
TOP FIELD (nT)

1

L$$
\begin{aligned}
& 7 \\
& 7 \\
& 7 \\
& 8 \\
& 8 \\
& 8
\end{aligned}
$$

8

8
$\begin{array}{cccc}\text { DAY TIME } & \text { STA. } \\ 190 & 132816 & 984 & 18\end{array}$ $\begin{array}{lll}190 & 132848 & 988\end{array}$ $\begin{array}{lll}190 & 133323 & 990\end{array}$ $190 \quad 133339 \quad 992$ $190 \quad 133359 \quad 994$ 190133429996 $190 \quad 133444 \quad 998$ 1901335001000 $\begin{array}{lll}190 & 133518 & 1002\end{array}$ $190 \quad 133534 \quad 1004$ $190 \quad 133547 \quad 1006$ $\begin{array}{llll}190 & 133602 & 1008 \quad 21\end{array}$ $\begin{array}{lllll}190 & 133615 & 1010 & 21 & 63\end{array}$ $\begin{array}{lllll}190 & 133629 & 1012 & 21 & 62\end{array}$ $\begin{array}{lllll}190 & 133644 & 1014 & 21 & 61\end{array}$ $\begin{array}{lllll}190 & 133715 & 1018 & 21 & 59\end{array}$ $\begin{array}{lllll}190 & 133732 & 1020 & 21 & 58\end{array}$ $\begin{array}{lllll}190 & 133747 & 1022 & 21 & 57\end{array}$ $\begin{array}{lllll}190 & 133801 & 1024 & 21 & 56\end{array}$ $\begin{array}{lllll}190 & 133814 & 1026 & 21 & 55\end{array}$ $\begin{array}{llllll}190 & 133829 & 1028 & 21 & 54 & ? 52138.2\end{array}$ $\begin{array}{llllll}190 & 133844 & 1030 & 21 & 53 & ? 51286\end{array}$ $\begin{array}{llllll}190 & 133859 & 1032 & 21 & 52 & ? 50917.8\end{array}$ $\begin{array}{llllll}190 & 133914 & 1034 & 21 & 51 & ? 50898\end{array}$ $\begin{array}{llllll}190 & 133929 & 1036 & 21 & 50 & ? 50970.6\end{array}$ $\begin{array}{llllll}190 & 133943 & 1038 & 21 & 49 & 51122.2\end{array}$ $\begin{array}{llllll}190 & 133958 & 1040 & 21 & 48 & 51321.4\end{array}$ $\begin{array}{llllll}190 & 134012 & 1042 & 21 & 47 & 51539.0\end{array}$ $\begin{array}{llllll}190 & 134028 & 1044 & 21 & 46 & 51675.4\end{array}$ $\begin{array}{llllll}190 & 134043 & 1046 & 21 & 45 & 51728.6\end{array}$ $\begin{array}{llllll}190 & 134100 & 1048 & 21 & 44 & 51717.6\end{array}$ $\begin{array}{llllll}190 & 134115 & 1050 & 21 & 43 & 51702.2\end{array}$ $\begin{array}{llllll}190 & 134129 & 1052 & 21 & 42 & 51697.0\end{array}$ $\begin{array}{llllll}190 & 134150 & 1054 & 21 & 41 & 51714.8\end{array}$ $\begin{array}{llllll}190 & 134204 & 1056 & 21 & 40 & 51712.8\end{array}$ $\begin{array}{llllll}190 & 134220 & 1058 & 21 & 39 & 51726.6\end{array}$ $\begin{array}{llllll}190 & 134234 & 1060 & 21 & 38 & 51804.0\end{array}$ $\begin{array}{llllll}190 & 134248 & 1062 & 21 & 37 & 51761.2\end{array}$ $\begin{array}{llllll}190 & 134301 & 1064 & 21 & 36 & 51502.6\end{array}$ $\begin{array}{llllll}190 & 134316 & 1066 & 21 & 35 & 51471.2\end{array}$ $\begin{array}{llllll}190 & 134330 & 1068 & 21 & 34 & 51532.8\end{array}$ $\begin{array}{llllll}190 & 134344 & 1070 & 21 & 33 & 51578.0\end{array}$ $\begin{array}{llllll}190 & 134357 & 1072 & 21 & 32 & 51606.2\end{array}$ $\begin{array}{llllll}190 & 134411 & 1074 & 21 & 31 & 51628.8\end{array}$ $\begin{array}{llllll}190 & 134425 & 1076 & 21 & 30 & 51642.0\end{array}$ $\begin{array}{llllll}190 & 134438 & 1078 & 21 & 29 & 51652.2\end{array}$ $\begin{array}{llllll}190 & 134453 & 1080 & 21 & 28 & 51661.2\end{array}$

\section{(Corr.)}

BOTTOM
(Diff.) (Uncorr $190 \quad 132829 \quad 986$ $\begin{array}{lllll}190 & 133658 & 1016 & 21 & 60\end{array}$ $\begin{array}{ll}51700.1 & -16.3\end{array}$ $51676.5-16.3$ $51597-15.8$ $51405.6-15.8$ $50710-15.8$ $50995.5-15.7$ $51418.7-15.7$ $51569.9-15.7$ $51644.9-15.7$ $51679.6-15.6$ $51703-15.6$ $51720.8-15.6$ $51740.8-15.6$ $51764.9-15.5$ $51805.5-15.5$ $51865.1-15.5$ $51969.9-15.5$ $52137.2-15.4$ $52451.4-15.4$ $52772.6-15.4$ $52779.2-15.4$ $52153.6-15.4$ $51301.3-15.3$ $50933.1-15.3$ $50913.3-15.3$ $50985.9-15.3$ $51137.4-15.2$ $51336.6-15.2$ $51554.2-15.2$ $51690.6-15.2$ $51743.7-15.1$ $51732.7-15.1$ $51717.3-15.1$ $51712.1-15.1$ $51729.8-15.0$ $51727.8-15.0$ $51741.6-15.0$ $51819-15.0$ $51776.1-14.9$ $51517.5-14.9$ $51486.1-14.9$ $51547.7-14.9$ $51592.9-14.9$ $51621-14.8$ $51643.6-14.8$ $51656.8-14.8$ $51667-14.8$ $51676-14.8$
Incorr) FIELD (nT) $51676.2 \quad 51691.5 \quad-15.3$ $51661.4 \quad 51676.7 \quad-15.3$ $51644.2 \quad 51659.5 \quad-15.3$ $51541.4 \quad 51556.1 \quad-14.7$ 51232.0 $? 50707$ $? 51159.6$ 51466.4 51582.2 51637.2 51663.0 51678.8 51689.4 51699.2 51710.0 51731.6 51762.2 51824.2

?51958.4

?52334.8

?53143.4

$? 53563$

?52282.8

?50606.2

?50449

50741.2

?50918.4

?50934.6

51307.4

51596.6

51783.0

51814.8

51721.0

?51762.2

51657.8

51726.4

51705.2

51687.8

?51937.6

?52008.8

?51180.2

51370.4

51523.2

51580.4

51608.6

51637.0

51651.4

51660.6

51668.0

$51246.7 \quad-14.7$

$50721.6-14.6$

$51174.2-14.6$

GRAD.

(nT/m)

$-46.2$

$-36.7$

$-26.2$

$-65.2$

$-258.7$

$51480.9 \quad-14.5 \quad 103.9$

$51596.7 \quad-14.5 \quad 45.9$

$51651.7-14.5 \quad 13.1$

$51677.4 \quad-14.4 \quad-1.6$

$51693.2 \quad-14.4 \quad-14.1$

$51703.8 \quad-14.4 \quad-25.9$

$51713.6 \quad-14.4 \quad-42.6$

$51724.3 \quad-14.3 \quad-64.6$

$\begin{array}{lll}51745.9 & -14.3 & -95.7\end{array}$

$51776.5 \quad-14.3 \quad-143.3$

$51838.4 \quad-14.2 \quad-213.4$

$51972.6 \quad-14.2 \quad-267.9$

$52349.0 \quad-14.2 \quad-165.9$

$53157.6 \quad-14.2 \quad 633.1$

$53577.1 \quad-14.1 \quad 1310.2$

$52296.9-14.1 \quad 237.1$

$50620.3 \quad-14.1 \quad-1114.4$

$50463.0 \quad-14.0 \quad-768.5$

$50755.2 \quad-14.0 \quad-257.1$

$50932.4 \quad-14.0 \quad-85.6$

$50948.6-14.0 \quad-307.5$

$\begin{array}{lll}51321.3 & -13.9 & -23.0\end{array}$

$51610.5 \quad-13.9 \quad 94.4$

$51796.9 \quad-13.9 \quad 176.4$

$\begin{array}{lll}51828.6 & -13.8 & 141.3\end{array}$

$51734.8 \quad-13.8 \quad 5.6$

$\begin{array}{lll}51776.0 & -13.8 & 98.4\end{array}$

$51671.5 \quad-13.7 \quad-64.3$

$51740.1 \quad-13.7 \quad 19.0$

$51718.9-13.7 \quad-12.5$

$\begin{array}{lll}51701.4 & -13.6 & -63.6\end{array}$

$51951.2 \quad-13.6 \quad 219.0$

$\begin{array}{lll}52022.4 & -13.6 & 405.9\end{array}$

$\begin{array}{lll}51193.8 & -13.6 & -528.5\end{array}$

$51383.9-13.5 \quad-165.2$

$51536.7 \quad-13.5 \quad-15.7$

$51593.9 \quad-13.5 \quad 3.9$

$51622.1 \quad-13.5 \quad 3.9$

$51650.4 \quad-13.4 \quad 13.4$

$51664.8-13.4 \quad 15.4$

$51674.0 \quad-13.4 \quad 13.8$

$51681.3 \quad-13.3 \quad 11.1$ 
TOP FIELD ( $\mathrm{nT}$ )

LINE DAY TIME STA. $X Y$ (Uncorr.) (Corr$$
8
$$$$
8
$$

8

\begin{tabular}{|c|c|c|c|c|c|c|c|c|c|c|c|}
\hline & & & & & & & & & 8 & , & 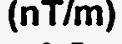 \\
\hline & & & & 27 & & 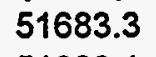 & -14.7 & & & & \\
\hline 90 & 134520 & 1084 & 2 & 26 & 671 & 39.1 & -14.7 & 1682.6 & 51695.9 & -13.3 & \\
\hline & 34539 & 1086 & & & 681.0 & .7 & -14.7 & & 0 & -13.2 & 2.8 \\
\hline & 34554 & 1088 & 21 & 24 & 684.2 & & -14.7 & 2.6 & 705.8 & -13.2 & 13.8 \\
\hline & 608 & & & & 688 ? & 8 & -14.6 & & .2 & & \\
\hline & & 1092 & 21 & 22 & 691.0 & .6 & -14.6 & & 11.8 & -13.2 & 25 \\
\hline & 638 & 94 & & & 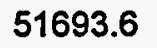 & 51708.2 & -146 & & & -13.1 & \\
\hline & & 1096 & 21 & & 1695.4 & 51710 & -14.6 & & 716.3 & -13.1 & 12.8 \\
\hline & 1700 & 1098 & & & 6 & 51712.1 & -14.5 & & 17.9 & -131 & 118 \\
\hline & & 100 & & & & 51714.3 & -14.5 & & & -13.0 & 11.8 \\
\hline & & & & & & 51715.7 & -14.5 & & & & 1.1 \\
\hline & & & & & & 51716.5 & -14.5 & & & -1 & 10.8 \\
\hline & & 106 & & & & & -14.4 & & & & .2 \\
\hline & 9 & 08 & & & & 51 & -14.4 & & & -12.9 & .9 \\
\hline & & 10 & & & & & -14.4 & & & -12.9 & 7.9 \\
\hline & 4848 & 12 & & & & & -14.4 & & & & 7.2 \\
\hline & & & & & & & -14.4 & & & -1 & .5 \\
\hline & 8 & 16 & & & & & -14 & & & & 10 \\
\hline & & & & & & & & & & -1 & .5 \\
\hline & 6 & 20 & & & & & -14.3 & & & & 5.9 \\
\hline & & & & & & & & & & -1 & 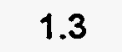 \\
\hline 30 & 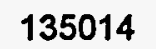 & 24 & & & & & -1 & & & & -0.3 \\
\hline & & & & & & & & & & -1 & -6.9 \\
\hline 90 & 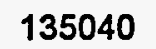 & 28 & & & & & -1 & & & -1 & -9.5 \\
\hline 90 & & & 2 & & & & & & & -1 & -19.0 \\
\hline 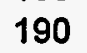 & & 32 & & & & & -1 & & & -1 & -36.7 \\
\hline & & & & & & & & & & & 0.4 \\
\hline 30 & 35 & 36 & & & & & -1 & & & -12.5 & 206.2 \\
\hline 90 & & 40 & 2 & & & & & & & & 0 \\
\hline 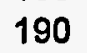 & & & & & & & .9 & & & & -88.9 \\
\hline 90 & & & 2 & & & & -9 & & & & \\
\hline 90 & & & & & & & -9 & & & -8 & -64.6 \\
\hline 190 & & 48 & 2 & & & & -9 & & & & \\
\hline 30 & & & & & & & -9 & & & -8 & -54.8 \\
\hline 190 & & 52 & 24 & & & & -9 & & & & \\
\hline & & & & & & & -9 & & & & 0.8 \\
\hline 190 & & & 2 & & & & -9 & & & & 7.9 \\
\hline & & & & & & & 0 & & & & $38 ?$ \\
\hline 90 & & & 2 & 1 & & & -9 & & & & 21.5 \\
\hline & & & & & & & & & & & 177 \\
\hline 3 & & & 2 & & & & -8 & & & & -17.7 \\
\hline & & & & & & & & & & & 17 \\
\hline 9 & & 168 & & & & & -8 & & & & 5.6 \\
\hline & & & & & & & & & & & \\
\hline & & 11 & & 6 & & & -8 & & & & .7 \\
\hline & & & & & & & & & & & \\
\hline & & 117 & & & & & -8 & & & & -15.7 \\
\hline & & & & & & & -8 & & & & r. \\
\hline & & & & & & .9 & & 51695.8 & 1702.6 & -6.8 & 18.0 \\
\hline
\end{tabular}

BOTTOM FIELD (nT) GRAD. 
TOP FIELD (nT)

BOTTOM FIELD (nT)

GRAD.

LINE DAY TIME STA. $X$ Y (Uncorr.) (Corr.) (Diff.) (Uncorr.) (Corr.) (Diff.) (nT/m)

\begin{tabular}{|c|c|c|c|c|c|c|c|c|c|c|c|}
\hline 190 & 140919 & 1182 & 24 & 21 & 51703.8 & 51711.8 & -8.0 & 51693.2 & 51699.9 & -6.7 & -17.4 \\
\hline 190 & 140932 & 1184 & 24 & 22 & 51701.0 & 51708.9 & -7.9 & 51689.0 & 51695.6 & -6.6 & -19.7 \\
\hline 190 & 140947 & 1186 & 24 & 23 & 51696.0 & 51703.8 & -7.8 & 51684.6 & 51691.1 & -6.5 & -18.7 \\
\hline 190 & 141011 & 1188 & 24 & 24 & 51690.0 & 51697.7 & -7.7 & 51679.0 & 51685.4 & -6.4 & -18.0 \\
\hline 190 & 141024 & 1190 & 24 & 25 & 51683.0 & 51690.6 & -7.6 & 1671.6 & 51677.9 & -6.3 & -18.7 \\
\hline 90 & 141041 & 1192 & 24 & 26 & 51677.2 & 51684.7 & -7.5 & 1664.8 & 671.0 & -6.2 & -20.3 \\
\hline 190 & 141055 & 1194 & 24 & 27 & 51665.2 & 1672.6 & -7.4 & 49.4 & & -6.1 & -25.9 \\
\hline 190 & 141108 & 1196 & 24 & 28 & 51656.4 & 51663.7 & -7.3 & 51635.6 & 1641.7 & -6.1 & -34.1 \\
\hline 190 & 141123 & 1198 & 24 & 29 & 51648.0 & 51655.3 & -7.3 & 51623.0 & 1629.0 & -6.0 & -41.0 \\
\hline 190 & 141136 & 1200 & 24 & 30 & 51639.6 & 51646.8 & -7.2 & 51613.2 & 1619.1 & -5.9 & -43.3 \\
\hline 190 & 141149 & 1202 & 24 & 31 & 51629.4 & 51636.5 & -7.1 & 51602.0 & 1607.8 & -5.8 & -44.9 \\
\hline 190 & 141204 & 1204 & 24 & 2 & 51615.4 & 1622.4 & -7.0 & 51587.2 & 592.9 & -5.7 & -46.2 \\
\hline 190 & 141218 & 1206 & 24 & 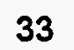 & 51587.2 & 94.1 & -6.9 & 561.2 & 566.9 & -5.7 & -42.6 \\
\hline 190 & 141232 & 1208 & 24 & 4 & 51537.0 & 51543.8 & -6.8 & 4.0 & 9.6 & -5.6 & -37.7 \\
\hline 190 & 141246 & 1210 & 24 & 5 & 51 & 55 & -6.8 & 9.6 & & -5.5 & -96.1 \\
\hline 190 & 141301 & 1212 & 24 & 6 & 51367.0 & 51373.7 & -6.7 & 99.4 & 04.8 & -5.4 & -438.7 \\
\hline 190 & 141316 & 1214 & 24 & 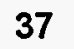 & ?51387.8 & 51394.4 & -6.6 & $? 50773.8$ & 50779.1 & -5.3 & -1006.6 \\
\hline 190 & 141334 & 1216 & 24 & 38 & 51 & 51510.3 & -6.5 & 250.6 & 55.8 & -5.2 & -415.1 \\
\hline 190 & 141350 & 1218 & 24 & 39 & 51590.0 & 51596.4 & -6.4 & 84.2 & 89.3 & -5.1 & -173.4 \\
\hline 190 & 141407 & 1220 & 24 & 40 & 51642.2 & 51648.5 & -6.3 & 1.0 & 76.0 & -5.0 & -116.7 \\
\hline 190 & 141423 & 1222 & 24 & 41 & 51 & 8.6 & -6.2 & 51703.2 & 8.1 & -4.9 & 4.1 \\
\hline 190 & 141438 & 1224 & 24 & 42 & 51 & 9.1 & -6.1 & 1.4 & 36.3 & -4.9 & -150.2 \\
\hline 190 & 141451 & 1226 & 24 & 43 & 51 & 13.2 & -6.0 & 85 & 39.8 & -4.8 & -364.3 \\
\hline 190 & 141507 & 1228 & 24 & 4 & 3.6 & 9.5 & -5.9 & 5.4 & 0.1 & -4.7 & 248.9 \\
\hline 190 & 141523 & 1230 & 24 & 45 & 4.2 & 51770 & -5.8 & 3.8 & 78.4 & -4.6 & 179.7 \\
\hline 190 & 141537 & 1232 & 24 & 46 & 2.0 & 51657.7 & -5.7 & 4.2 & 18.7 & -4.5 & 102.0 \\
\hline 190 & 141552 & 1234 & 24 & 47 & 51489.2 & 51494.8 & -5.6 & 34.8 & 89.2 & -4.4 & -7.2 \\
\hline 190 & 141606 & 1236 & 24 & 48 & 51273.2 & 51278.7 & -5.5 & 1.4 & 5.8 & -4.4 & -52.1 \\
\hline 190 & 141621 & 1238 & 24 & 49 & ?50983.8 & 50989.3 & -5.5 & $? 50761.2$ & 65.5 & -4.3 & -364.9 \\
\hline 190 & 141638 & 1240 & 24 & 50 & 17.6 & 50723 & -5.4 & $? 50055.8$ & 60.0 & -4.2 & -1084.9 \\
\hline 190 & 141653 & 1242 & 24 & 51 & 2.8 & 8.1 & -5.3 & 58 & $\$ 2.1$ & -4.1 & -991.5 \\
\hline 190 & 141708 & 1244 & 24 & 52 & 59.4 & 4.6 & -5.2 & 07.2 & 11.2 & -4.0 & -1233.1 \\
\hline 190 & 141722 & 1246 & 24 & 53 & 14.8 & 9.9 & -5.1 & 3.2 & 7.1 & -3.9 & -1855.1 \\
\hline 190 & 141737 & 1248 & 24 & 54 & 37.2 & 2.2 & -5.0 & 34.6 & 8.4 & -3.8 & 9635.1 \\
\hline 190 & 141751 & 1250 & 24 & 55 & 25.2 & 54130.1 & -4.9 & 52.6 & 56.3 & -3.7 & \#\#\# \\
\hline 190 & 141807 & 1252 & 24 & 56 & ?54383.2 & 54388 & -4.8 & 89.4 & 93.1 & -3.7 & 6895.4 \\
\hline 190 & 141826 & 1254 & 24 & 57 & $? 53431.2$ & 53435.9 & -4.7 & ?53679.6 & 83.1 & -3.5 & 407.2 \\
\hline 190 & 141842 & 1256 & 24 & 58 & 52632.6 & 52637.2 & -4.6 & ?52392.6 & 96.1 & -3.5 & -393.4 \\
\hline 190 & 141901 & 1258 & 24 & 59 & 52172.8 & 52177.3 & -4.5 & 87.4 & 10.7 & -3.3 & -303.9 \\
\hline 190 & 141914 & 1260 & 24 & 60 & 51 & 51977 & -4.4 & & 2.1 & -3.3 & -219.3 \\
\hline 190 & 141930 & 1262 & 24 & 61 & 51 & 9.3 & -4.3 & 1.8 & 5.0 & -3.2 & -152.8 \\
\hline 190 & 141945 & 1264 & 24 & 68 & 51 & 15.2 & -4.2 & 3.0 & 46.1 & -3.1 & -111.5 \\
\hline 190 & 142000 & 1266 & 24 & 63 & 51777.8 & 51781.9 & -4.1 & 51723.8 & 51726.8 & -3.0 & -88.5 \\
\hline 190 & 142015 & 1268 & 24 & 64 & 51747.0 & 51751 & -4.0 & 51709.6 & 51712.5 & -2.9 & -61.3 \\
\hline 190 & 142029 & 1270 & 24 & 00 & 51728.4 & 51732.4 & -4.0 & 35.4 & 51698.2 & -2.8 & -54.1 \\
\hline 190 & 142042 & 1272 & 24 & 66 & 51703.8 & 51707.7 & -3.9 & 78.8 & 51681.6 & -2.8 & -41.0 \\
\hline 190 & 142056 & 1274 & 24 & 67 & & 6.8 & -3.8 & 5.6 & 558.3 & -2.7 & -28.5 \\
\hline 190 & 142110 & 1276 & 24 & 68 & & 51623.1 & -3.7 & 51613.8 & 51616.4 & -2.6 & -9.2 \\
\hline 190 & 142125 & 1278 & D & 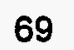 & 51514.6 & 51518.2 & -3.6 & 51548.4 & 51550.9 & -2.5 & 55.4 \\
\hline
\end{tabular}


TOP FIELD (nT)

BOTTOM FIELD (nT)

GRAD.

LINE DAY TIME STA. $X \quad Y$ (Uncorr.) (Corr.) (Diff.) (Uncorr.) (Corr.) (Diff.) (nT/m)

9

$\begin{array}{cccc}9 & 190 & 142220 & 1286 \\ 10 & 190 & 142921 & 1288\end{array}$

$10 \quad 190 \quad 142955 \quad 1290 \quad 27$

$\begin{array}{lllll}10 & 190 & 143014 & 1292 & 27\end{array}$

$\begin{array}{lllll}10 & 190 & 143029 & 1294\end{array}$

$\begin{array}{llll}10 & 190 & 143044 & 1296 \\ 10 & 190 & 143058 & 1298\end{array}$

$10 \quad 1901431111300$

$\begin{array}{lllll}10 & 190 & 143125 & 1302\end{array}$

$\begin{array}{lllll}10 & 190 & 143141 & 1304\end{array}$

$\begin{array}{lllll}10 & 190 & 143156 & 1306\end{array}$

$\begin{array}{lllll}10 & 190 & 143211 & 1308\end{array}$

10

$\begin{array}{lllll}10 & 190 & 143239 & 1312\end{array}$

$\begin{array}{lllll}10 & 190 & 143255 & 1314\end{array}$

$\begin{array}{llll}10 & 190 & 143309 & 1316\end{array}$

$\begin{array}{lllll}10 & 190 & 143325 & 1318\end{array}$

$\begin{array}{lllll}10 & 190 & 143343 & 1320\end{array}$

$\begin{array}{lllll}10 & 190 & 143358 & 1322\end{array}$

$\begin{array}{lllll}10 & 190 & 143413 & 1324\end{array}$

$\begin{array}{lllll}10 & 190 & 143428 & 1326\end{array}$

$\begin{array}{llll}10 & 190 & 143443 & 1328\end{array}$

$\begin{array}{lllll}10 & 190 & 143501 & 1330\end{array}$

$\begin{array}{lllll}10 & 190 & 143516 & 1332\end{array}$

$\begin{array}{llll}10 & 190 & 143533 & 1334\end{array}$

$\begin{array}{llll}10 & 190 & 143601 & 1336\end{array}$

$\begin{array}{llll}10 & 190 & 143619 & 1338\end{array}$

$\begin{array}{llll}10 & 190 & 143636 & 1340\end{array}$

$\begin{array}{llll}10 & 190 & 143652 & 1342\end{array}$

$\begin{array}{llll}10 & 190 & 143705 & 1344\end{array}$

$10 \quad 1901437191346$

$\begin{array}{lllll}10 & 190 & 143734 & 1348\end{array}$

$\begin{array}{llll}10 & 190 & 143753 & 1350\end{array}$

$\begin{array}{llll}10 & 190 & 143809 & 1352\end{array}$

$\begin{array}{llll}10 & 190 & 143825 & 1354\end{array}$

$\begin{array}{llll}10 & 190 & 143843 & 1356\end{array}$

$\begin{array}{lllll}10 & 190 & 143900 & 1358\end{array}$

$\begin{array}{lllll}10 & 190 & 143914 & 1360\end{array}$

$\begin{array}{lllll}10 & 190 & 143931 & 1362\end{array}$

$\begin{array}{lllll}10 & 190 & 143946 & 1364\end{array}$

$\begin{array}{lllll}10 & 190 & 143958 & 1366\end{array}$

$\begin{array}{lllll}10 & 190 & 144012 & 1368\end{array}$

$\begin{array}{lllll}10 & 190 & 144025 & 1370\end{array}$

$\begin{array}{lllll}10 & 190 & 144040 & 1372\end{array}$

$\begin{array}{lllll}10 & 190 & 144054 & 1374\end{array}$

$10 \quad 190 \quad 1441141376$

\section{0}

$\begin{array}{ll}24 & 71 \\ 24 & 72\end{array}$

2473

2773

$\begin{array}{lll}27 & 72 & ? 51246.8\end{array}$

$\begin{array}{lll}27 & 71 & ? 50669.6\end{array}$

$\begin{array}{lll}27 & 70 & 51162.8\end{array}$

$\begin{array}{lll}27 & 69 & 51493.4\end{array}$

$\begin{array}{lll}27 & 68 & 51616.6\end{array}$

$\begin{array}{lll}27 & 67 & 51676.0\end{array}$

$\begin{array}{lll}27 & 66 & 51708.4\end{array}$

$\begin{array}{lll}27 & 65\end{array}$

$\begin{array}{lll}27 & 64 \\ & 51\end{array}$

2763

2762

2761

2760

2759

2758

2757

2756

$\begin{array}{llll}27 & 55 & ? 53241.8\end{array}$

$\begin{array}{lll}27 & 54 & ? 52057.6\end{array}$

$\begin{array}{lll}27 & 53 & ? 51406.8\end{array}$

$\begin{array}{lll}27 & 52 & ? 51144.6\end{array}$

$\begin{array}{lll}27 & 51 & 350882.6\end{array}$

$\begin{array}{lll}27 & 50 & ? 50763.6\end{array}$

$\begin{array}{lll}27 & 49 & ? 50963.6\end{array}$

$\begin{array}{lll}27 & 48 & 51223.0\end{array}$

$\begin{array}{lll}27 & 47\end{array}$

51427.2

51589.4

51719.0

51790.8

51815.4

51775.2

51692.0

51694.2

51685.6

51564.6

51596.4

51718.0

51588.4

51575.6

51659.0

51772.0

51843.0

51755.4

51651.0
$51289.5-3.5$

$\begin{array}{lll}51085.7 & -3.5\end{array}$

$\begin{array}{lll}51439 & -3.4\end{array}$

$51607.1-3.3$

$\begin{array}{lll}51595.3 & -0.7\end{array}$

$\begin{array}{lll}51247.3 & -0.5\end{array}$

$\begin{array}{lll}50670 & -0.4\end{array}$

$\begin{array}{lll}51163.1 & -0.3\end{array}$

$\begin{array}{lll}51493.6 & -0.2\end{array}$

$\begin{array}{lll}51616.8 & -0.2\end{array}$

$\begin{array}{lll}51676.1 & -0.1\end{array}$

$\begin{array}{lll}51708.4 & 0.0\end{array}$

51737.10 .1

$\begin{array}{lll}51764.8 & 0.2\end{array}$

$\begin{array}{lll}51796.9 & 0.3\end{array}$

$\begin{array}{lll}51842.6 & 0.4\end{array}$

$51918.3 \quad 0.5$

52063.80 .6

$52301.6 \quad 0.6$

$52770.5 \quad 0.7$

$\begin{array}{lll}53472.2 & 0.8\end{array}$

53959.30 .9

53240.81 .0

52056.51 .1

$51405.6 \quad 1.2$

51143.31 .3

50881.21 .4

50762.11 .5

50961.91 .7

51221.21 .8

51425.31 .9

51587.42 .0

51716.92 .1

$51788.6 \quad 2.2$

51813.22 .2

51772.82 .4

51689.52 .5

51691.72 .5

51682.92 .7

51561.82 .8

51593.62 .8

51715.12 .9

51585.43 .0

51572.53 .1

$51655.8 \quad 3.2$

51768.73 .3

51839.63 .4

51751.93 .5

51647.43 .6
251399.4

51092.0

51381.8

51548.2

51568.0

?50983.6

?50649.8

$? 51320$

51550.2

51636.4

51678.4

51701.6

51720.2

51739.4

51758.2

51788.0

51839.4

51954.0

$? 52157$

?52652.4

?53939.8

255592.6

?53493.2

?51463.8

51170.8

51014.0

?50450.2

?50195

?50762.6

51167.8

51411.6

51615.2

51811.4

51904.0

?51972.4

?51920.8

51634.4

51751.2

$? 51822$

?51229.4

?51161.8

?51783.2

?51362.2

51469.2

51591.2

51707.2

?51747.6

?51596.6

?51509.8
$51401.8-2.4$

$\begin{array}{ll}51094.4 & -2.4\end{array}$

$51384.1-2.3$

$51550.4 \quad-2.2$

$\begin{array}{ll}51567.8 & 0.2\end{array}$

50983.20 .4

$50649.3 \quad 0.5$

51319.4

51549.5

51635.6

51677.5

51700.7

51719.2

51738.3

51757.0

51786.7

51838.0

51952.5

52155.5

52650.8

53938.1

55590.8

53491.3

51461.8

51168.7

51011.8

50447.9

50192.6

50760.1

51165.2

51408.9

51612.4

51808.5

51901.0

51969.3

51917.6

51631.1

51747.8

51818.5

51225.8

51158.2

51779.5

51358.4

51465.3

51587.2

51703.1

51743.5

51592.4

51505.5

185.9

16.1

$-88.2$

$-91.2$

$-43.6$

$-431.5$

$-32.5$

257.7

$0.7 \quad 93.1$

$0.8 \quad 32.5$

$0.9 \quad 3.9$

$0.9 \quad-11.1$

$1.0 \quad-27.9$

$1.1-42.0$

$1.2-63.9$

$1.3-90.2$

$1.4-130.2$

$1.5-181.0$

$1.5-238.0$ 
TOP FIELD ( $\mathrm{nT}$ )

BOTTOM FIELD (nT)

GRAD.

\begin{tabular}{|c|c|c|c|c|c|c|c|c|c|c|c|c|}
\hline & & & TA. & X & $\mathbf{Y}$ & irr.) & 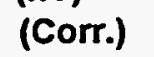 & - & orr.) & orr.) & (Diff.) & 1107 \\
\hline 10 & 90 & 137 & 378 & 27 & 28 & 51615.4 & 51611.7 & 3.7 & 51543.0 & 51538.5 & 4.5 & -118.7 \\
\hline 10 & 190 & 144154 & 380 & 27 & 27 & 51621.4 & 51617.6 & 3.8 & 51587.0 & 51582.4 & 4.6 & -56.4 \\
\hline 10 & 190 & 144212 & 382 & 27 & 26 & 51637.0 & 51633.1 & 3.9 & 51625.4 & 51620.7 & 4.7 & -19.0 \\
\hline 10 & 190 & 144225 & 1384 & 27 & 25 & 51653.6 & 51649.6 & 4.0 & 651.4 & 51646.7 & 4.7 & -3.6 \\
\hline 10 & 190 & 144240 & 1386 & 27 & 24 & 51664.8 & 51660.7 & 4.1 & 51667.8 & 51663.0 & 4.8 & 4.9 \\
\hline 10 & 190 & 144253 & 1388 & 27 & 23 & 51672.6 & 51668.4 & 4.2 & 51679.4 & 51674.5 & 4.9 & 11.1 \\
\hline 10 & 190 & 144307 & 1390 & 27 & 22 & 51679.6 & 51675.3 & 4.3 & 51687.2 & 51682.2 & 5.0 & 12.5 \\
\hline 1 & 190 & 144323 & 1392 & 27 & 21 & 51685.8 & 51681.4 & 4.4 & 51693.4 & 51688.3 & 5.1 & 12.5 \\
\hline 16 & 190 & 144338 & 1394 & 27 & 20 & 51690.0 & 51685.6 & 4.4 & 51693.2 & 51688.0 & 5.2 & 5.2 \\
\hline 10 & 190 & 144356 & 1396 & 27 & 19 & 51683.8 & 51679.2 & 4.6 & 56.4 & 51551.1 & 5.3 & -208.9 \\
\hline 10 & 190 & 144411 & 1398 & 27 & 18 & 51686.8 & 51682.2 & 4.6 & 4.6 & 51669.2 & 5.4 & -20.0 \\
\hline 10 & 190 & 144425 & 1400 & 27 & 17 & 51698.8 & 51694.1 & 4.7 & 9.8 & 704.4 & 5.4 & 18.0 \\
\hline 10 & 190 & 144440 & 1402 & 27 & 16 & 51704.8 & 1700 & 4.8 & 5.8 & 51710.3 & 5.5 & 18.0 \\
\hline 10 & 190 & 144454 & 1404 & 27 & 15 & 51709.8 & 51704.9 & 4.9 & 0.8 & 51715.2 & 5.6 & 18.0 \\
\hline 10 & 190 & 144508 & 1406 & 27 & 14 & 517 & 51707.6 & 5.0 & & 51713.9 & 5.7 & 11.5 \\
\hline 10 & 190 & 144523 & 1408 & 27 & 13 & 51714.0 & 51708.9 & 5.1 & 0.0 & 51714.2 & 5.8 & 9.8 \\
\hline 10 & 190 & 144546 & 1410 & 27 & 12 & 517 & 51710.8 & 5.2 & 517 & 51720.5 & 5.9 & 17.1 \\
\hline 10 & 190 & 144559 & 1412 & 27 & 11 & 51 & 11.1 & 5.3 & 3.0 & 17.0 & 6.0 & 10.8 \\
\hline 10 & 190 & 144613 & 1414 & 27 & 10 & 51 & 51711.2 & 5.4 & 1.8 & 25.7 & 6.1 & 24.9 \\
\hline 10 & 190 & 144627 & 1416 & 27 & 9 & 51711.2 & 51705.7 & 5.5 & 6.6 & 10.5 & 6.1 & 8.9 \\
\hline 10 & 190 & 144643 & 1418 & 27 & 8 & 51701.4 & 95.8 & 5.6 & & 95.2 & 6.2 & 0.0 \\
\hline 10 & 190 & 144657 & 1420 & 27 & 7 & 51677.0 & 51671.4 & 5.6 & 2.0 & 45.7 & 6.3 & -41.0 \\
\hline 10 & 190 & 144712 & 1422 & 27 & 6 & 51667.0 & 51661.3 & 5.7 & & 91.6 & 6.4 & -277.0 \\
\hline 10 & 190 & 144727 & 1424 & 27 & 5 & $? 5$ & 51891.2 & 5.8 & 3.4 & 66.9 & 6.5 & 4059.7 \\
\hline 10 & 190 & 144747 & 1426 & 27 & 4 & $? 52$ & 52020.9 & 5.9 & $? 5$ & 84.4 & 6.6 & 2039.7 \\
\hline 10 & 190 & 144802 & 1428 & 27 & 3 & 51 & 05.4 & 6.0 & $? 51$ & 51722.9 & 6.7 & -134.1 \\
\hline 10 & 190 & 144 & 1430 & 27 & 2 & 51 & 8.5 & 6.1 & 6.0 & 19.2 & 6.8 & -96.1 \\
\hline 10 & 190 & 144834 & 1432 & 27 & 1 & 51 & 30.4 & 6.2 & 0.8 & 9.9 & 6.9 & -81.6 \\
\hline 10 & 190 & 144849 & 1434 & 27 & 0 & 515 & 51576.5 & 6.3 & 5.8 & 58.8 & 7.0 & -191.8 \\
\hline 71 & 190 & 151143 & 1438 & 30 & 0 & 51 & 97.1 & 12.7 & & 18.0 & 13.6 & -128.2 \\
\hline 11 & 190 & 151159 & 1440 & 30 & 1 & & 45.5 & 13.1 & & 6.3 & 14.1 & -62.6 \\
\hline 11 & 190 & 151213 & 1442 & 30 & 2 & & 51677.8 & 13.2 & & 5.1 & 14.1 & -35.7 \\
\hline 11 & 190 & 151226 & 1444 & 30 & 3 & 51707.0 & 51693.7 & 13.3 & 4.0 & 79.8 & 14.2 & -21.3 \\
\hline 11 & 190 & 151239 & 1446 & 30 & 4 & 51707.4 & 51694.1 & 13.3 & 4.0 & 579.7 & 14.3 & -22.0 \\
\hline 1 & 190 & 15 & 1448 & 30 & 5 & 51 & 51686.2 & 13.4 & 1.6 & 51667.3 & 14.3 & -29.5 \\
\hline & 190 & 15 & 1450 & 30 & 6 & 51 & 88.9 & 13.5 & 8.0 & 73.6 & 14.4 & -23.6 \\
\hline 11 & 190 & 151 & 1452 & 30 & 7 & 51 & 0.3 & 13.5 & .6 & 3.1 & 14.5 & -10.2 \\
\hline 11 & 190 & 151338 & 1454 & 30 & 8 & 51 & 6.8 & 13.6 & & 9.9 & 14.5 & 6.6 \\
\hline 11 & 190 & 151351 & 1456 & 30 & 9 & & 51729.3 & 13.7 & & $\$ 0.0$ & 14.6 & 19.0 \\
\hline 11 & 190 & 151405 & 1458 & 30 & 10 & 51746.6 & 51732.8 & 13.8 & & 37.1 & 14.7 & 8.5 \\
\hline 11 & 190 & 151418 & 1460 & 30 & 11 & 51744.2 & 51730.4 & 13.8 & & 35.4 & 14.8 & 9.8 \\
\hline 11 & 190 & 151 & 1462 & 30 & 12 & & 51726.7 & 13.9 & 9.8 & 51735.0 & 14.8 & 15.1 \\
\hline 11 & 190 & 151447 & 1464 & 30 & 13 & & 51720 & 14.0 & 5.8 & 730.9 & 14.9 & 19.3 \\
\hline & 190 & 151502 & 1466 & 30 & 14 & 51 & 51711.6 & 14.0 & & 24.4 & 15.0 & 22.6 \\
\hline & 190 & 151514 & 1468 & 30 & 15 & 51718.0 & 51703.9 & 14.1 & & 14.4 & 15.0 & 18.7 \\
\hline . & 190 & 151528 & 1470 & 30 & 16 & 51708.8 & 51694.6 & 14.2 & 51719.8 & 51704.7 & 15.1 & 18.0 \\
\hline 11 & 190 & 151541 & 1472 & 30 & 17 & 51703.4 & 51689.2 & 14.2 & 51710.0 & 51694.8 & 15.2 & 10.8 \\
\hline$\because$ & 190 & 151554 & 1474 & 30 & 18 & 51698.8 & 51684.5 & 14.3 & 51705.0 & 51689.8 & 15.2 & 10.2 \\
\hline & 10 & & 1476 & 30 & 19 & 51696.8 & 51682.5 & 14.3 & 51702.6 & 51687.3 & 15.3 & 9.5 \\
\hline
\end{tabular}


TOP FIELD (nT)

LINE DAY TIME STA.

$\begin{array}{llll}11 & 190 & 151620 & 1478\end{array}$

$\begin{array}{llll}11 & 190 & 151634 & 1480\end{array}$

$11 \quad 190 \quad 1516471482$

$\begin{array}{lllll}11 & 190 & 151700 & 1484\end{array}$

$11 \quad 1901517151486$

$\begin{array}{lllll}11 & 190 & 151749 & 1488\end{array}$

$11190151803 \quad 1490$

$\begin{array}{lllll}11 & 190 & 151817 & 1492\end{array}$

$11 \quad 1901518321494$

$\begin{array}{lllll}11 & 190 & 151846 \quad 1496\end{array}$

$\begin{array}{lllll}11 & 190 & 151928 & 1498\end{array}$

$\begin{array}{llll}11 & 190 & 152000 & 1500\end{array}$

$11 \quad 1901520211502$

$11 \quad 1901520341504$

$11190152048 \quad 1506$

$11190152103 \quad 1508$

11

11

11

11

11

11

11

11

11

11

11

11

11

11

11

11

11

11

11

11

11

11

11

11

11

11

11

11

11

11

11

11

11 $\begin{array}{lll}190 & 152118 & 1510\end{array}$

$\begin{array}{lll}190 & 152131 & 1512\end{array}$

1901521451514

1901521591516

1901522121518

1901522251520

$\begin{array}{lll}190 & 152238 & 1522\end{array}$

1901522511524

1901523051526

$\begin{array}{lll}190 & 152319 & 1528\end{array}$

1901523311530

1901523451532

1901524001534

$190152413 \quad 1536$

$\begin{array}{lll}190 & 152438 & 1538\end{array}$

1901524521540

$190 \quad 1525061542$

1901525201544

1901525341546

1901525521548

$\begin{array}{lll}190 & 152622 \quad 1550\end{array}$

$\begin{array}{lll}190 & 152643 \quad 1552\end{array}$

$\begin{array}{lll}190 & 152658 & 1554\end{array}$

$\begin{array}{ll}190 & 1527121556\end{array}$

$\begin{array}{lll}190 & 152725 & 1558\end{array}$

$\begin{array}{lll}190 & 152746 \quad 1560\end{array}$

$\begin{array}{lll}190 & 152837 & 1562\end{array}$

$\begin{array}{lll}190 & 152848 & 1564\end{array}$

$\begin{array}{lll}190 & 152903 & 1566\end{array}$

1901529151568

$190 \quad 1529281570$

$\begin{array}{lll}190 & 152940 & 1572\end{array}$

1901529521574
$X Y$

3020

$\begin{array}{lll}30 & 21\end{array}$

3022

$\begin{array}{ll}30 & 23\end{array}$

3024

3025

3026

$\begin{array}{ll}30 & 27\end{array}$

3028

$30 \quad 29$

$\begin{array}{lll}30 & 30\end{array}$

$\begin{array}{lll}30 & 31\end{array}$

$30 \quad 32$

$\begin{array}{ll}30 & 33\end{array}$

$\begin{array}{ll}30 & 34\end{array}$

$\begin{array}{lll}30 & 35\end{array}$

3036

$\begin{array}{ll}30 & 37\end{array}$

$\begin{array}{lll}30 & 38\end{array}$

$\begin{array}{lll}30 & 39\end{array}$

$\begin{array}{lll}30 & 40\end{array}$

$\begin{array}{lll}30 & 41\end{array}$

$\begin{array}{lll}30 & 42\end{array}$

$\begin{array}{lll}30 & 43\end{array}$

$\begin{array}{lll}30 & 44\end{array}$

$30 \quad 45$

$\begin{array}{lll}30 & 46\end{array}$

$\begin{array}{lll}30 & 47\end{array}$

$\begin{array}{lll}30 & 48\end{array}$

$\begin{array}{lll}30 & 49\end{array}$

3050

3051

3052

3053

3054

3055

3056

$\begin{array}{ll}30 & 57 \\ 30 & 58\end{array}$

3059

3060

3061

3062

3063

$30 \quad 64$

3065

$30 \quad 66$

3067

3068
(Uncorr) (Corr.)

(Corr.) (Diff.) (Uncorr.)

$\begin{array}{lll}51679.6 & 14.4 & 51698.4\end{array}$

$51677.1 \quad 14.5$

$51673.5 \quad 14.5$

$51667.6 \quad 14.6$

$51661.3 \quad 14.7$

$51657 \quad 14.8$

$51641.5 \quad 14.9$

51634.815 .0

51658.215 .0

51814.315 .1

52102.515 .3

52293.315 .5

$? 52117.8$

?52308.8

52086.6

51825.0

51683.8

51636.4

51633.6

51650.2

51642.0

51633.4

51675.2

51731.2

51766.4

51773.8

51765.6

51735.8

51658.0

51514.8

51279.6

?51028.4

$52071 \quad 15.6$

$51809.4 \quad 15.6$

$51668.1 \quad 15.7$

$51620.6 \quad 15.8$

$51617.8 \quad 15.8$

$51634.3 \quad 15.9$

$51626 \quad 16.0$

51617.416 .0

51659.116 .1

$51715 \quad 16.2$

51750.216 .2

$51757.5 \quad 16.3$

51749.316 .3

$51719.4 \quad 16.4$

51641.516 .5

$51498.3 \quad 16.5$

$51263 \quad 16.6$

$51011.7 \quad 16.7$

$50787.6 \quad 16.8$

$50819.5 \quad 16.9$

51113.116 .9

51440.217 .0

52079.517 .1

$53067.4 \quad 17.2$

$\begin{array}{lll}53791.7 & 17.3\end{array}$

53370.217 .4

52881.317 .5

52384.917 .5

$52114.8 \quad 17.6$

$51968.9 \quad 17.7$

51986.6

51907.4

51848.4

51806.4

51772.2

51747.8

51718.8

51674.2
51889.517 .9

51830.418 .0

51788.318 .1

51754.118 .1

$51729.6 \quad 18.2$

$\begin{array}{lll}51700.6 \quad 18.2 & \end{array}$

51655.918 .3

\section{6}

51687.6

51680.4

51669.8

51657.6

51636.2

51597.6

51569.8

?51662.2

?52208.4

?52650.4

$? 52137.2$

51731.6

51568.0

51504.6

?51466.2

51566.6

51580.6

51568.8

51669.4

51776.6

51820.8

51801.4

51805.2

51797.6

51722.6

51569.8

51211.8

?50799.8

?50252.2

?50261.4

50945.2

?51091.8

$? 51790.2$

?56052.4

?55369.2

?58507

?52809

?52232.2

51987.2

51878.6

51838.2

51798.8

51767.2

51744.0

51726.6

51704.0

51669.4

IELD (nT)

(Corr.)

51683.0 (Diff.)

51678.2

51672.1

51664.8

51654.2

51641.8

51620.3

51581.6

51553.8

51646.1

52192.1

52633.9

52120.6

51715.0

51551.3

51487.8

51449.3

51549.7

51563.6

51551.7

51652.3 
TOP FIELD (nT)

BOTTOM FIELD (nT)

GRAD.

LINE DAY TIME STA. $X \quad Y$ (Uncorr.) (Corr.) (Diff.) (Uncorr.) (Corr.) (Diff.) (nT/m)

$\begin{array}{lllllllllllll}12 & 195 & 93546 & 1580 & 33 & 68 & 51590.2 & 51582.1 & 8.1 & 51615.6 & 51608.5 & 7.1 & 41.6\end{array}$

$\begin{array}{llllllllll}12 & 195 & 93614 & 1582 & 33 & 67 & 51666.8 & 51658.9 & 7.9\end{array}$

$\begin{array}{lllllllll}12 & 195 & 93627 & 1584 & 33 & 66 & 51702.0 & 51694.1 & 7.9\end{array}$

$\begin{array}{lllllllll}12 & 195 & 93642 & 1586 & 33 & 65 & 51733.8 & 51726 & 7.8\end{array}$

$\begin{array}{lllllllll}12 & 195 & 93710 & 1588 & 33 & 64 & 51765.8 & 51758.1 & 7.7\end{array}$

$\begin{array}{lllllllll}12 & 195 & 93722 & 1590 & 33 & 63 & 51799.0 & 51791.3 & 7.7\end{array}$

$\begin{array}{lllllllll}12 & 195 & 93735 & 1592 & 33 & 62 & 51843.0 & 51835.4 & 7.6\end{array}$

$\begin{array}{llllllllll}12 & 195 & 93747 & 1594 & 33 & 61 & 51901.6 & 51894.1 & 7.5\end{array}$

$\begin{array}{lllllllll}12 & 195 & 93829 & 1596 & 33 & 60 & 51997.6 & 51990.2 & 7.4\end{array}$

$\begin{array}{lllllllll}12 & 195 & 93842 & 1598 & 33 & 59 & 52181.6 & 52174.3 & 7.3\end{array}$

$\begin{array}{lllllllll}12 & 195 & 93855 & 1600 & 33 & 58 & 52519.6 & 52512.4 & 7.2\end{array}$

$\begin{array}{lllllllll}12 & 195 & 93909 & 1602 & 33 & 57 & 253083.4 & 53076.2 & 7.2\end{array}$

$\begin{array}{lllllllll}12 & 195 & 94047 & 1604 & 33 & 56 & 253215.2 & 53208.4 & 6.8\end{array}$

$\begin{array}{lllllllll}12 & 195 & 94101 & 1606 & 33 & 55 & ? 52461.6 & 52454.9 & 6.7\end{array}$

$\begin{array}{lllllllll}12 & 195 & 94115 & 1608 & 33 & 54 & ? 51631.4 & 51624.8 & 6.6\end{array}$

$\begin{array}{lllllllll}12 & 195 & 94130 & 1610 & 33 & 53 & ? 51279.6 & 51273 & 6.6\end{array}$

$\begin{array}{lllllllll}12 & 195 & 94144 & 1612 & 33 & 52 & 51217.4 & 51210.9 & 6.5\end{array}$

$\begin{array}{lllllllll}12 & 195 & 94158 & 1614 & 33 & 51 & 51042.6 & 51036.1 & 6.5\end{array}$

$\begin{array}{lllllllll}12 & 195 & 94210 & 1616 & 33 & 50 & ? 50965.6 & 50959.2 & 6.4\end{array}$

$\begin{array}{lllllllll}12 & 195 & 94223 & 1618 & 33 & 49 & 51102.6 & 51096.3 & 6.3\end{array}$

$\begin{array}{lllllll}12 & 195 & 94234 & 1620 & 33 & 48 & 51302.6\end{array}$

$\begin{array}{lllllll}12 & 195 & 94246 & 1622 & 33 & 47 & 51476.4\end{array}$

$\begin{array}{llllllll}12 & 195 & 94259 & 1624 & 33 & 46 & 51629.6\end{array}$

$\begin{array}{llllllll}12 & 195 & 94319 & 1626 & 33 & 45 & 51753.2\end{array}$

$\begin{array}{llllllll}12 & 195 & 94331 & 1628 & 33 & 44 & 51815.0\end{array}$

$\begin{array}{lllllll}12 & 195 & 94344 & 1630 & 33 & 43 & 51833.8\end{array}$

$\begin{array}{lllllll}12 & 195 & 94358 & 1632 & 33 & 42 & 51798.8\end{array}$

$\begin{array}{llllllll}12 & 195 & 94410 & 1634 & 33 & 41 & 51729.6\end{array}$

$\begin{array}{lllllll}12 & 195 & 94426 & 1636 & 33 & 40 & 51684.8\end{array}$

$\begin{array}{lllllll}12 & 195 & 94438 & 1638 & 33 & 39 & 51687.6\end{array}$

$\begin{array}{lllllll}12 & 195 & 94450 & 1640 & 33 & 38 & 51797.6\end{array}$

$\begin{array}{lllllll}12 & 195 & 94502 & 1642 & 33 & 37 & ? 52013.4\end{array}$

$\begin{array}{lllllll}12 & 195 & 94550 & 1644 & 33 & 36 & 51885.0\end{array}$

$\begin{array}{lllllll}12 & 195 & 94611 & 1646 & 33 & 35 & 51811.6\end{array}$

$\begin{array}{lllllll}12 & 195 & 94623 & 1648 & 33 & 34 & 51773.6\end{array}$

$\begin{array}{lllllll}12 & 195 & 94644 & 1650 & 33 & 33 & 51648.2\end{array}$

$\begin{array}{lllllll}12 & 195 & 94657 & 1652 & 33 & 32 & 51629.4\end{array}$

$\begin{array}{lllllll}12 & 195 & 94709 & 1654 & 33 & 31 & 51642.4\end{array}$

$\begin{array}{lllllll}12 & 195 & 94721 & 1656 & 33 & 30 & 51647.2\end{array}$

$\begin{array}{lllllll}12 & 195 & 94733 & 1658 & 33 & 29 & 51642.6\end{array}$

$\begin{array}{lllllll}12 & 195 & 94745 & 1660 & 33 & 28 & 51639.4\end{array}$

$\begin{array}{lllllll}12 & 195 & 94802 & 1662 & 33 & 27 & 51639.8\end{array}$

$\begin{array}{lllllll}12 & 195 & 94815 & 1664 & 33 & 26 & 51641.6\end{array}$

$\begin{array}{lllllll}12 & 195 & 94836 & 1666 & 33 & 25 & 51640.2\end{array}$

$\begin{array}{lllllll}12 & 195 & 94849 & 1668 & 33 & 24 & 51638.6\end{array}$

$\begin{array}{lllllll}12 & 195 & 94903 & 1670 & 33 & 23 & 51633.0\end{array}$

$\begin{array}{lllllll}12 & 195 & 94915 & 1672 & 33 & 22 & 51625.8\end{array}$

$51296.3 \quad 6.3$

51470.26 .2

51623.46 .2

51747.16 .1

$51809 \quad 6.0$

$51827.8 \quad 6.0$

$51792.9 \quad 5.9$

$51723.7 \quad 5.9$

$51679 \quad 5.8$

$51681.8 \quad 5.8$

$51791.9 \quad 5.7$

$52007.7 \quad 5.7$

51668.8

$\begin{array}{llll}51682.6 & 51675.7 & 6.9 & -31.8\end{array}$

$\begin{array}{llll}51710.6 & 51703.7 & 6.9 & -38.0\end{array}$

$\begin{array}{llll}51740.8 & 51734.0 & 6.8 & -41.0\end{array}$

$\begin{array}{llll}51762.4 & 51755.7 & 6.7 & -60.0\end{array}$

$\begin{array}{llll}51794.0 & 51787.3 & 6.7 & -80.3\end{array}$

$\begin{array}{llll}51835.8 & 51829.2 & 6.6 & -107.9\end{array}$

$\begin{array}{llll}51902.8 & 51896.4 & 6.4 & -155.4\end{array}$

$\begin{array}{llll}52058.2 & 52051.8 & 6.4 & -202.3\end{array}$

$\begin{array}{llll}\text { ?52464.2 } & 52457.9 & 6.3 & -90.8\end{array}$

$\begin{array}{llll}253579.8 & 53573.5 & 6.3 & 813.8\end{array}$

$\begin{array}{llll}? 53957.2 & 53951.3 & 5.9 & 1216.4\end{array}$

$\begin{array}{llll}252355.8 & 52350.0 & 5.8 & -173.4\end{array}$

$\begin{array}{llll}250892.8 & 50887.0 & 5.8 & -1210.8\end{array}$

$\begin{array}{llll}250840 & 50834.3 & 5.7 & -720.7\end{array}$

$\begin{array}{lllll}? 51449.8 & 51444.1 & 5.7 & 381.0\end{array}$

$\begin{array}{llll}? 50907.8 & 50902.2 & 5.6 & -221.0\end{array}$

?50689.2 $50683.6 \quad 5.6 \quad-453.1$

$\begin{array}{llll}51028.0 & 51022.5 & 5.5 & -122.3\end{array}$

$\begin{array}{llll}51330.8 & 51325.3 & 5.5 & 46.2\end{array}$

$\begin{array}{llll}51481.6 & 51476.2 & 5.4 & 8.5\end{array}$

$\begin{array}{llll}51692.6 & 51687.2 & 5.4 & 103.3\end{array}$

$\begin{array}{llll}51890.2 & 51884.9 & 5.3 & 224.6\end{array}$

$\begin{array}{llll}51962.0 & 51956.8 & 5.2 & 241.0\end{array}$

$\begin{array}{llll}\text { ?52018 } & 52012.8 & 5.2 & 302.0\end{array}$

$\begin{array}{llll}? 51955 & 51949.9 & 5.1 & 256.1\end{array}$

$\begin{array}{llll}51747.0 & 51741.9 & 5.1 & 28.5\end{array}$

$\begin{array}{llll}51671.0 & 51666.0 & 5.0 & -22.6\end{array}$

$\begin{array}{llll}51656.8 & 51651.8 & 5.0 & -50.5\end{array}$

$\begin{array}{llll}51848.6 & 51843.7 & 4.9 & 83.6\end{array}$

$\begin{array}{llll}258506.6 & 58501.7 & 4.9 & 10644.6\end{array}$

$51879.6 \quad 5.4$

$? 51852.6$

51847.9

$4.7 \quad-53.1$

51806.25 .4

?52087.8

52083.2

4.6

452.8

$51768.3 \quad 5.3$

$? 51975.8$

51971.3

4.5

331.5

51550.0

51545.6

4.4

$-161.0$

51624.25 .2

51561.2

51556.8

$4.4 \quad-111.8$

$51637.3 \quad 5.1$

51598.6

51594.3

4.3

$-71.8$

51642.15 .1

$51637.6 \quad 5.0$

$51634.5 \quad 4.9$

$51634.9 \quad 4.9$

$51636.8 \quad 4.8$

$51635.5 \quad 4.7$

$51633.9 \quad 4.7$

51613.0

51608.7

$4.3 \quad-56.1$

$\begin{array}{llll}51617.0 & 51612.8 & 4.2 & -42.0\end{array}$

$51624.8 \quad 51620.6$

4.2

51629.8

51625.7

4.1

$-23.9$

51645.4

51641.3

4.1

$-16.4$

51646.4

51642.4

4.0

6.2

51643.2

51639.3

$51628.4 \quad 4.6$

51641.2

51637.3

3.9

10.2

51637.6

51633.8

3.9

7.5

$51621.2 \quad 4.6$

51626.0

51622.2

3.8

13.4

$51611.9 \quad 4.5$

51624.0

51620.3

3.7

19.3

15.7

$\begin{array}{lllllllll}12 & 195 & 94946 & 1676 & 33 & 20 & 51606.6 & 51602.2 & 4.4\end{array}$ 
TOP FIELD (nT)

BOTTOM FIELD (nT)

GRAD.

\begin{tabular}{|c|c|c|c|c|c|c|c|c|c|c|c|c|}
\hline IINE & DAY & TIME & STA. & $x$ & $\mathbf{Y}$ & (Uncorr.) & (Corr.) & (Diff.) & rrr.) & (Corr.) & Diff.) & $T / m)$ \\
\hline 12 & 195 & 94959 & 1678 & 33 & 19 & 51600.2 & 51595.8 & 4.4 & 51647.8 & 51644.1 & 3.7 & 78.0 \\
\hline 12 & 195 & 95014 & 1680 & 33 & 18 & 51593.8 & 51589.5 & 4.3 & 51624.4 & 51620.8 & 3.6 & 50.2 \\
\hline 12 & 195 & 95028 & 1682 & 33 & 17 & 51600.8 & 51596.6 & 4.2 & 51633.6 & 51630.1 & 3.5 & 53.8 \\
\hline 12 & 195 & 95039 & 1684 & 33 & 16 & 51616.4 & 51612.2 & 4.2 & 51658.4 & 51654.9 & 3.5 & 68.9 \\
\hline 12 & 195 & 95051 & 1686 & 33 & 15 & 51643.6 & 51639.5 & 4.1 & 51706.6 & 51703.2 & 3.4 & 103.3 \\
\hline 12 & 195 & 95106 & 1688 & 3 & 4 & 51664.2 & 51660.1 & 4.1 & 51707.4 & 51704.0 & 3.4 & 70.8 \\
\hline 12 & 195 & 95120 & 1690 & 3 & 3 & 51687.8 & 51683.8 & 4.0 & 51723.0 & 51719.7 & 3.3 & 57.7 \\
\hline 12 & 195 & 95132 & 1692 & 3 & 12 & 51717.8 & 51713.8 & 4.0 & 51763.8 & 51760.5 & 3.3 & 75.4 \\
\hline 12 & 195 & 95144 & 1694 & 3 & 11 & 51739.6 & 51735.7 & 3.9 & 93.6 & 51790.4 & 3.2 & 88.5 \\
\hline 12 & 195 & 95157 & 1696 & 3 & 10 & 51747.4 & 51743.5 & 3.9 & 51793.6 & 51790.4 & 3.2 & 75.7 \\
\hline 12 & 195 & 95211 & 1698 & 3 & 9 & 51743.8 & 51740 & 3.8 & 0.8 & 51757.7 & 3.1 & 27.9 \\
\hline 12 & 195 & 95223 & 1700 & 3 & 8 & 517 & 51735.7 & 3.7 & .6 & 51772.5 & 3.1 & 59.3 \\
\hline 12 & 195 & 95235 & 1702 & 3 & 7 & & 51721.5 & 3.7 & & 51725.0 & 3.0 & 4.6 \\
\hline 12 & 195 & 95258 & 1704 & 33 & 6 & 517 & 51714.4 & 3.6 & .8 & 1.9 & 2.9 & 11.1 \\
\hline 12 & 195 & 95309 & 1706 & 3 & 5 & 517 & 51707.7 & 3.5 & .0 & 1.1 & 2.9 & 4.6 \\
\hline 12 & 195 & 95321 & 1708 & 3 & 4 & 51 & 51697.1 & 3.5 & .8 & 6.0 & 2.8 & -3.0 \\
\hline 12 & 195 & 95332 & 1710 & 33 & 3 & 51 & 51682.4 & 3.4 & & & 2.8 & -13.4 \\
\hline 12 & 195 & 95345 & 1712 & 3 & 2 & & 57.4 & 3.4 & & 9.1 & 2.7 & -31.1 \\
\hline 12 & 195 & 95357 & 1714 & 33 & 1 & 51 & 51618.9 & 3.3 & .2 & 3.5 & 2.7 & -75.4 \\
\hline 12 & 195 & 95412 & 1716 & 33 & 0 & & 51540.7 & 3.3 & & & 2.6 & -283.9 \\
\hline 13 & 195 & 95844 & 1720 & 36 & 0 & & 51456.3 & 2.9 & & & 2.3 & -271.1 \\
\hline 13 & 195 & 95903 & 1722 & 6 & 1 & 51 & 51589.9 & 2.5 & & & 1.9 & -102.6 \\
\hline 13 & 195 & 95916 & 1724 & 36 & 2 & & 48.3 & 2.5 & 2.0 & 0.1 & 1.9 & -47.2 \\
\hline 13 & 195 & 95929 & 1726 & 36 & 3 & & 7.2 & 2.4 & & & 1.9 & -22.3 \\
\hline 13 & 195 & 95942 & 1728 & 36 & 4 & .8 & 0.4 & 2.4 & & & 1.8 & -10.2 \\
\hline 13 & 195 & 95955 & 1730 & 6 & 5 & & 7.2 & 2.4 & & & 1.8 & 1.3 \\
\hline 13 & 195 & 100007 & 1732 & 36 & 6 & & 32.6 & 2.4 & & & 1.8 & 24.6 \\
\hline 13 & 195 & 100019 & 1734 & 36 & 7 & & 43.5 & 2.3 & & & 1.8 & 31.5 \\
\hline 13 & 195 & 100032 & 1736 & 36 & 8 & & 3.9 & 2.3 & & & 1.8 & 43.3 \\
\hline 13 & 195 & 100045 & 1738 & 36 & 9 & & 51760.9 & 2.3 & & & 1.7 & 79.0 \\
\hline 13 & 195 & 100057 & 1740 & 36 & 10 & & 51756.3 & 2.3 & & .9 & 1.7 & 16.4 \\
\hline 13 & 195 & 100109 & 1742 & 36 & 11 & & 51735.5 & 2.3 & & & 1.7 & 44.3 \\
\hline 13 & 195 & 100123 & 1744 & 36 & 12 & & 81.8 & 2.2 & & & 1. & 56.7 \\
\hline 13 & 195 & 100146 & 1746 & 6 & 13 & & 32.4 & 2.2 & 7.4 & & 1.7 & 99.7 \\
\hline 13 & 195 & 100208 & 1748 & 36 & 14 & & 3.6 & 2.2 & 3.6 & 2.0 & 1.6 & 324.3 \\
\hline 13 & 195 & 100230 & 1750 & 36 & 15 & & & 2.1 & & & 1.6 & 352.1 \\
\hline 13 & 195 & 100243 & 1752 & 36 & 16 & & 37.5 & 2.1 & & & 1.6 & 262.9 \\
\hline 13 & 195 & 100258 & 1754 & 36 & 17 & & 65.9 & 2.1 & & & 1.5 & 188.9 \\
\hline 13 & 195 & 100310 & 1756 & 36 & 18 & & 51252.8 & 2.0 & & & 1.5 & 113.4 \\
\hline 13 & 195 & 100324 & 1758 & 36 & 19 & & 51348.4 & 2.0 & & 7.5 & 1.5 & 79.7 \\
\hline 13 & 195 & 100337 & 1760 & 36 & 20 & & 44.2 & 2.0 & & 7.5 & 1.5 & 37.4 \\
\hline 13 & 195 & 100350 & 1762 & 36 & 27 & & 0.4 & 2.0 & & 7.7 & 1.5 & 27.5 \\
\hline 13 & 195 & 100402 & 1764 & 36 & 22 & & 6.2 & 2.0 & & & 1.4 & 12.8 \\
\hline 13 & 195 & 100416 & 1766 & 36 & 23 & & 51594.1 & 1.9 & & 7.2 & 1.4 & 4.3 \\
\hline 13 & 195 & 100428 & 1768 & 36 & 24 & & 51617.7 & 1.9 & & & 1.4 & 1.3 \\
\hline 13 & 195 & 100441 & 1770 & 36 & 25 & 51634.6 & 51632.7 & 1.9 & 4.0 & 51632.6 & 1.4 & -1.0 \\
\hline 13 & 195 & 100455 & 1772 & 36 & 26 & 51646.0 & 51644.1 & 1.9 & 2.8 & 51641.4 & 1.4 & -5.2 \\
\hline 13 & 195 & 100508 & 1774 & 36 & 27 & 51650.2 & 51648.4 & 1.8 & 6.8 & 45.5 & 1.3 & -5.6 \\
\hline 13 & 195 & 100523 & 776 & 36 & 28 & 51650.2 & 51648.4 & 1.8 & 51640.2 & 51638.9 & 1.3 & -16 \\
\hline
\end{tabular}


TOP FIELD (nT)

BOTTOM FIELD (nT)

GRAD.

LINE DAY TIME STA. $X \quad Y$ (Uncorr.) (Corr.) (Diff.) (Uncorr.) (Corr.) (Diff.) (nT/m)

$\begin{array}{lllllllllllll}13 & 195 & 100535 & 1778 & 36 & 29 & 51650.6 & 51648.8 & 1.8 & 51663.6 & 51662.3 & 1.3 & 21.3\end{array}$

$\begin{array}{llllllllllllll}13 & 195 & 100547 & 1780 & 36 & 30 & 51645.2 & 51643.4 & 1.8 & 51653.4 & 51652.1 & 1.3 & 13.4\end{array}$

$\begin{array}{lllllllllllll}13 & 195 & 100559 & 1782 & 36 & 31 & 51626.8 & 51625 & 1.8 & 51616.2 & 51614.9 & 1.3 & -17.4\end{array}$

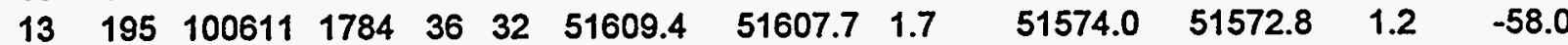

$\begin{array}{lllllllllllll}13 & 195 & 100625 & 1786 & 36 & 33 & 51598.2 & 51596.5 & 1.7 & 51582.0 & 51580.8 & 1.2 & -26.6\end{array}$

$\begin{array}{lllllllllllll}13 & 195 & 100639 & 1788 & 36 & 34 & 51575.0 & 51573.3 & 1.7 & 51609.0 & 51607.8 & 1.2 & 55.7\end{array}$

$\begin{array}{llllllllllllll}13 & 195 & 100652 & 1790 & 36 & 35 & 51537.0 & 51535.3 & 1.7 & 51476.6 & 51475.4 & 1.2 & -99.0\end{array}$

$\begin{array}{lllllllllllll}13 & 195 & 100707 & 1792 & 36 & 36 & 51510.6 & 51509 & 1.6 & 51421.2 & 51420.1 & 1.1 & -146.6\end{array}$

$\begin{array}{lllllllllllll}13 & 195 & 100721 & 1794 & 36 & 37 & 51506.6 & 51505 & 1.6 & 51415.0 & 51413.9 & 1.1 & -150.2\end{array}$

$\begin{array}{lllllllllllll}13 & 195 & 100734 & 1796 & 36 & 38 & 51493.2 & 51491.6 & 1.6 & 51379.8 & 51378.7 & 1.1 & -185.9\end{array}$

$\begin{array}{llllllllllllll}13 & 195 & 100748 & 1798 & 36 & 39 & 51507.0 & 51505.4 & 1.6 & 51442.4 & 51441.3 & 1.1 & -105.9\end{array}$

$\begin{array}{lllllllllllll}13 & 195 & 100803 & 1800 & 36 & 40 & 51576.4 & 51574.9 & 1.5 & ? 51475.4 & 51474.3 & 1.1 & -165.6\end{array}$

$\begin{array}{lllllllll}13 & 195 & 100816 & 1802 & 36 & 41 & 51669.0 & 51667.5 & 1.5\end{array}$

$\begin{array}{lllllllll}13 & 195 & 100829 & 1804 & 36 & 42 & 51684.8 & 51683.3 & 1.5\end{array}$

$\begin{array}{lllllllll}13 & 195 & 100842 & 1806 & 36 & 43 & 51673.8 & 51672.3 & 1.5\end{array}$

$\begin{array}{lllllllll}13 & 195 & 100904 & 1808 & 36 & 44 & 51633.8 & 51632.4 & 1.4\end{array}$

$\begin{array}{lllllllll}13 & 195 & 100944 & 1810 & 36 & 45 & 51552.8 & 51551.4 & 1.4\end{array}$

$\begin{array}{lllllllll}13 & 195 & 101001 & 1812 & 36 & 46 & 51470.6 & 51469.3 & 1.3\end{array}$

$\begin{array}{lllllllll}13 & 195 & 101013 & 1814 & 36 & 47 & 51389.6 & 51388.3 & 1.3\end{array}$

$\begin{array}{lllllllll}13 & 195 & 101027 & 1816 & 36 & 48 & 51257.6 & 51256.3 & 1.3\end{array}$

$\begin{array}{lllllllll}13 & 195 & 101040 & 1818 & 36 & 49 & 51057.4 & 51056.1 & 1.3\end{array}$

$\begin{array}{lllllllll}13 & 195 & 101054 & 1820 & 36 & 50 & ? 50826.8 & 50825.6 & 1.2\end{array}$

$\begin{array}{lllllll}13 & 195 & 101108 & 1822 & 36 & 51 & ? 50761.8\end{array}$

$\begin{array}{lllllll}13 & 195 & 101121 & 1824 & 36 & 52 & ? 50895.8\end{array}$

$\begin{array}{lllllll}13 & 195 & 101134 & 1826 & 36 & 53 & ? 51197\end{array}$

$\begin{array}{lllllll}13 & 195 & 101148 & 1828 & 36 & 54 & ? 51886\end{array}$

$\begin{array}{lllllll}13 & 195 & 101201 & 1830 & 36 & 55 & ? 52991\end{array}$

$\begin{array}{lllllll}13 & 195 & 101220 & 1832 & 36 & 56 & ? 53775.4\end{array}$

$\begin{array}{lllllll}13 & 195 & 101233 & 1834 & 36 & 57 & ? 53540.2\end{array}$

$\begin{array}{lllllll}13 & 195 & 101248 & 1836 & 36 & 58 & ? 53143.2\end{array}$

$\begin{array}{lllllll}13 & 195 & 101301 & 1838 & 36 & 59 & 52587.2\end{array}$

$\begin{array}{lllllll}13 & 195 & 101313 & 1840 & 36 & 60 & 52243.2\end{array}$

50760.61 .2

$50894.6 \quad 1.2$

$51195.8 \quad 1.2$

$51884.9 \quad 1.1$

52989.91 .1

53774.31 .1

$53539.1 \quad 1.1$

$53142.2 \quad 1.0$

52586.21 .0

51760.0

51685.4

51776.2

$? 51749.6$

51618.0

51759.0

1.0

149.2

51483.8

51421.2

51684.4

1.0

1.0

167.9

189.8

51258.0

51775.2

1.0

106.9

50910.2

51617.1

0.9

21.6

?50413.4

51482.9

0.9

51.8

?50305.

51420.4

0.8

0.7

?50472.2

51257.2

0.8

$-241.3$

?50626.6

50909.4

0.8

$-677.7$

$\begin{array}{lll}50304.4 & 0.8 & -748.5\end{array}$

$? 51724.6$

50471.5

0.7

$-694.4$

?54813

50625.9

0.7

$-935.1$

?55453.8

51723.9

0.7

$-264.6$

0.7

2986.9

?56606.2 56605.6

0.6

2751.5

?53295.2 53294.6

0.6

5026.2

$? 52435.8$

52435.2

249.2

?52083.6

52083.0

0.6

$-248.2$

$\begin{array}{lllllll}13 & 195 & 101330 & 1842 & 36 & 61 & 52041.0\end{array}$

52242.21 .0

51936.2

51935.7

$-261.6$

$\begin{array}{llllllll}13 & 195 & 101351 & 1844 & 36 & 62 & 51933.8\end{array}$

51932009

51862.6

51862.1

$-171.8$

$\begin{array}{lllllll}13 & 195 & 101403 & 1846 & 36 & 63 & 51860.8\end{array}$

$51859.9 \quad 0.9$

51806.90 .9

51801.6

51801.1

$-116.7$

51764.8

51692.4

51715.8

51764.3

51692.2

51728.70 .5

$\begin{array}{llllllll}14 & 195 & 101759 & 1854 & 39 & 66 & 51757.6\end{array}$

51757.10 .5

$\begin{array}{llllll}14 & 195 & 101843 & 1856 & 39 & 65\end{array}$

51757.6

51736.2

51715.7

51758.6

51736.1

51787.20 .4

51787.0

51758.6

0.6

0.5

0.5

$-97.0$

$\begin{array}{ll}0.5 & -97.0 \\ 0.5\end{array}$

$0.2-20.0$

$0.1-22.0$

$0.1-35.1$

$0.0-47.5$

51837.6

51787.0

$0.0-64.3$

$\begin{array}{lllllll}14 & 195 & 101929 & 1860 & 39 & 63 & 51893.8\end{array}$

$\begin{array}{lll}51893.5 & 0.3\end{array}$

$\begin{array}{llllllll}14 & 195 & 101949 & 1862 & 39 & 62 & 51986.6\end{array}$

51986.30 .3

51916.2

51837.6

0.0

$-92.1$

51994.8

51916.3

$-0.1$

$-115.4$

252156.4

51995.1

$-0.3$

$-145.6$

52289.40 .0

?52577.4

52156.8

$-0.4$

$-218.0$

$\begin{array}{llllllll}14 & 195 & 102312 & 1868 & 39 & 59 & 52656.6\end{array}$

52656.60 .0

?53482.4

52577.8

$-129.8$

354392.6

53482.8

$-0.4$

391.2

$\begin{array}{lllllll}14 & 195 & 102350 & 1872 & 39 & 57 & ? 53541.2\end{array}$

$53541.3-0.1$

?53885.4

$54393.0-0.4$

1395.7

874.1 
TOP FIELD (nT)

\begin{tabular}{|c|c|c|c|c|c|c|c|c|c|c|c|c|}
\hline MF & 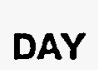 & rIM & & & & Uncorr.) & (Corr & ... & (Uncorr.) & (Corr.) & (Diff.) & (nT/m) \\
\hline T & 100 & & 876 & 39 & 55 & & 52482.2 & -0.2 & ?52453.8 & 52454.3 & -0.5 & -46.2 \\
\hline 14 & 195 & 102438 & 1878 & 39 & 54 & & 51604.6 & -0.2 & ?50613.2 & 50613.7 & -0.5 & 1624.9 \\
\hline 14 & 195 & 102453 & 380 & 39 & 53 & 01.8 & & -0.2 & & 812.1 & & \\
\hline 14 & 195 & 102505 & 882 & 39 & 52 & 91.4 & & -0.2 & & 860.4 & -0.6 & \\
\hline 14 & 195 & 102519 & 884 & 39 & 51 & & 50813.3 & -0.3 & & 172.0 & -0.6 & 51.8 \\
\hline 14 & 195 & 102533 & 1886 & 39 & 50 & $? 50$ & & -0.3 & & 555.2 & -0.6 & -582.3 \\
\hline 14 & 195 & 102545 & 888 & 39 & 49 & & & -0.3 & & 044.8 & -0.6 & -138.4 \\
\hline 14 & 195 & 102558 & 1890 & 39 & 48 & & & -0.3 & & & -0.6 & -15.4 \\
\hline 14 & 195 & 102610 & 1892 & 39 & 47 & & & & & & -0.7 & 65.6 \\
\hline 14 & 195 & 102622 & 1894 & 39 & 46 & & & -0.4 & & & -0.7 & -147.5 \\
\hline 14 & 195 & 102635 & 1896 & 39 & 45 & 51 & & -0.4 & & 5 & -0.7 & 7.9 \\
\hline 14 & 195 & 102646 & 1898 & 39 & 44 & & & -0.4 & & & -0.7 & 1.6 \\
\hline 14 & 195 & 102659 & 1900 & 39 & 43 & & & -0.4 & & 3.7 & -0.7 & -263.0 \\
\hline 14 & 195 & 102711 & 1902 & 39 & 42 & & & -0.5 & & 6.0 & -0.8 & 9.2 \\
\hline 14 & 195 & 102723 & 1904 & 39 & 41 & & & -0.5 & & & -0.8 & 156.1 \\
\hline 14 & 195 & 102741 & & 39 & 40 & & & -0.5 & & & -0.8 & 5.1 \\
\hline 14 & 195 & 55 & 8 & 39 & 39 & & & & & & & -347.2 \\
\hline 14 & 195 & 02807 & 1910 & 39 & 38 & & & -0 & & & -0.9 & -177.4 \\
\hline 14 & 195 & 102821 & 912 & 39 & 37 & & & -0 & & & -0.9 & 52.8 \\
\hline 14 & 195 & 102833 & 1914 & 39 & 36 & & & -0. & & 7.7 & -0.9 & -112.5 \\
\hline 14 & 195 & 102857 & 1916 & 39 & 35 & & & -0 & & & -0.9 & 125.6 \\
\hline 14 & 195 & 102910 & 1918 & 39 & 34 & & & -0 & & & -0 & 4.1 \\
\hline 14 & 195 & 102933 & 1920 & 39 & 33 & & & -0 & & & & 5.4 \\
\hline 14 & 195 & 02945 & 922 & 39 & 32 & & & -0 . & & & -1 & 4.3 \\
\hline 14 & 195 & & & 39 & 31 & & & -0 & & & & \\
\hline 14 & $19 t$ & & & 39 & 30 & & & -0 & & 66.2 & -1 & 17.0 \\
\hline 14 & 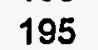 & & & 39 & 29 & & & & & & & \\
\hline 14 & 10 & & & 39 & 28 & & & & & & & \\
\hline$\cdots$ & 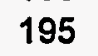 & & & 39 & 27 & & & -0 & & & -1 & \\
\hline$i$ & 105 & 354 & 34 & 39 & 26 & & & -6 & & & -1 & 6 \\
\hline 14 & 195 & 103106 & 6 & 39 & 25 & & & -0 & & & -1 & 5.2 \\
\hline 14 & 95 & 103118 & & 39 & 24 & & & -0.9 & & & -1.2 & -12.1 \\
\hline 14 & 195 & 103129 & 1940 & 39 & 23 & & & -0.9 & & & -1.2 & -16.4 \\
\hline 14 & 195 & & & 39 & 22 & & & & & & -1.2 & -23.9 \\
\hline 14 & 195 & & & 39 & 21 & & & & & 8.8 & -1 & -32.5 \\
\hline 14 & 195 & & & 39 & 20 & & & & & & -1 & -42.3 \\
\hline 14 & & & & 39 & 19 & & & & & & & \\
\hline 14 & 195 & 48 & & 39 & 18 & & & 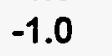 & & 3.5 & & \\
\hline 14 & 19 & & & 39 & 17 & & & -1 & & & -1.3 & 3.6 \\
\hline 14 & & & & 39 & 16 & & & & & & -1.4 & -45920 \\
\hline 14 & 195 & 103348 & 1956 & 39 & 15 & & & -1 & 2.6 & 4.0 & -1.4 & 19 \\
\hline 14 & 195 & 1034 & & 39 & 14 & & & -1 & & 95.4 & -1 & 1.6 \\
\hline 14 & 19 & & & 39 & 13 & & & -1 & & 27.3 & -1 & 1182.0 \\
\hline 14 & & & & 39 & 12 & & & -1 & & & -1 & \\
\hline$\cdots$ & 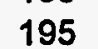 & & & 39 & 11 & & & -1 & & & -1 & \\
\hline 7 & 19 & & 1966 & 39 & 10 & & & -1 & & & & \\
\hline 14 & 195 & & 1968 & 39 & 9 & & & -1 & & & & 125.6 \\
\hline 14 & . & & & 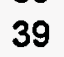 & 8 & & & -1.3 & & & & 75.4 \\
\hline$\because$ & 195 & 03539 & 2 & 39 & 7 & 1.8 & 51733.1 & -1.3 & 51760.2 & 51761.8 & -1.6 & 46.6 \\
\hline
\end{tabular}

BOTTOM FIELD (nT) GRAD.

(Uncorr.) (Corr.) (Diff.) (nT/m)

$\begin{array}{llll}? 50613.2 & 50613.7 & -0.5 & -1624.9\end{array}$

$\begin{array}{llll}250811.6 & 50812.1 & -0.5 & -639.7\end{array}$

$\begin{array}{llll}? 50859.8 & 50860.4 & -0.6 & -215.7\end{array}$

$\begin{array}{llll}250171.4 & 50172.0 & -0.6 & -1051.8\end{array}$

$\begin{array}{lllll}50554.6 & 50555.2 & -0.6 & -582.3\end{array}$

$\begin{array}{llll}51044.2 & 51044.8 & -0.6 & -138.4\end{array}$

$\begin{array}{llll}51278.0 & 51278.6 & -0.6 & -15.4\end{array}$

$\begin{array}{llll}51325.8 & 51326.5 & -0.7 & -65.6\end{array}$

$\begin{array}{llll}51296.4 & 51297.1 & -0.7 & -147.5\end{array}$

$\begin{array}{llll}51343.8 & 51344.5 & -0.7 & -107.9\end{array}$

$\begin{array}{llll}51273.8 & 51274.5 & -0.7 & -201.6\end{array}$

$\begin{array}{llll}51535.2 & 51536.0 & -0.8 & 9.2\end{array}$

$\begin{array}{llll}51670.8 & 51671.6 & -0.8 & 156.1\end{array}$

$\begin{array}{llll}51348.2 & 51349.0 & -0.8 & -155.1\end{array}$

$\begin{array}{lllll}251105.2 & 51106.0 & -0.8 & -347.2\end{array}$

$\begin{array}{llll}51184.8 & 51185.7 & -0.9 & -177.4\end{array}$

$\begin{array}{llll}? 51341.4 & 51342.3 & -0.9 & 52.8\end{array}$

$\begin{array}{llll}51296.8 & 51297.7 & -0.9 & -112.5\end{array}$

$\begin{array}{llll}51550.4 & 51551.3 & -0.9 & 125.6\end{array}$

$\begin{array}{llll}51574.0 & 51574.9 & -0.9 & 54.1\end{array}$

$51610.8 \quad 51611.8 \quad-1.0 \quad 35.4$

$51631.8 \quad 51632.8 \quad-1.0 \quad 24.3$

$51649.0 \quad 51650.0 \quad-1.0 \quad 16.1$

$51665.2 \quad 51666.2 \quad-1.0 \quad 17.0$

$51672.0 \quad 51673.1 \quad-1.1 \quad 9.5$

$51679.4 \quad 51680.5 \quad-1.1 \quad 16.1$

$51680.4 \quad 51681.5 \quad-1.1 \quad 4.6$

$\begin{array}{llll}51659.6 & 51660.7 & -1.1 & -5.2\end{array}$

$51633.6 \quad 51634.8 \quad-1.2 \quad-12.1$

$51593.4 \quad 51594.6 \quad-1.2 \quad-16.4$

$\begin{array}{llll}51527.4 & 51528.6 & -1.2 & -23.9\end{array}$

$51407.6 \quad 51408.8 \quad-1.2 \quad-32.5$

$\begin{array}{llll}51198.0 & 51199.2 & -1.2 & -42.3\end{array}$

$\begin{array}{llll}50851.0 & 50852.3 & -1.3 & -30.8\end{array}$

$\begin{array}{llll}249992.2 & 49993.5 & -1.3 & -276.1\end{array}$

$\begin{array}{llll}\text { ?48268.2 } & 48269.5 & -1.3 & -963.6\end{array}$

$? 19516.2 \quad 19517.6 \quad-1.4 \quad-45920.0$

$\begin{array}{llll}58592.6 & 58594.0 & -1.4 & 19259.0\end{array}$

$? 52094$

$\begin{array}{llll}251325.8 & 51327.3 & -1.5 & 1182.0\end{array}$

?51770.8 $51772.3 \quad-1.5 \quad 667.2$

$\begin{array}{llll}251873.2 & 51874.7 & -1.5 & 385.6\end{array}$

$\begin{array}{llll}51846.0 & 51847.5 & -1.5 & 215.7\end{array}$

$\begin{array}{llll}51784.8 & 51786.3 & -1.5 & 75.4\end{array}$
$51674.8 \quad 51675.9 \quad-1.1 \quad 1.6$ 
TOP FIELD (nT)

BOTTOM FIELD (nT)

GRAD.

\begin{tabular}{|c|c|c|c|c|c|c|c|c|c|c|c|c|}
\hline$x_{-1}$ & $A Y$ & & & & & & & & & & & 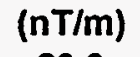 \\
\hline 14 & 195 & 103550 & 1974 & 39 & 6 & 21.0 & 51722.4 & -1.4 & 5.6 & & & \\
\hline 14 & 195 & 103602 & 1976 & 39 & 5 & 04.8 & 51706.2 & -1.4 & & .8 & -1.6 & 7.2 \\
\hline 14 & 195 & 103613 & 1978 & 39 & 4 & & 886.4 & -1.4 & & & -1.6 & 5.6 \\
\hline 14 & 195 & 103626 & 1980 & 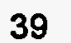 & 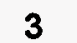 & & 4.4 & -1.4 & & & -1.6 & -17.0 \\
\hline 14 & 195 & 103639 & 1982 & 39 & 2 & & & -1.4 & & & -1.7 & -37.7 \\
\hline 14 & 19 & 103651 & 1984 & 39 & 1 & & & -1.5 & & & -1.7 & -89.2 \\
\hline 14 & 195 & 103704 & 1986 & 39 & 0 & & 33.5 & -1.5 & & & -1.7 & 13.4 \\
\hline 15 & 195 & 103946 & 1990 & 42 & 0 & & 30.4 & -1.6 & & & -1.8 & 54.8 \\
\hline 15 & 195 & 104239 & 1992 & 42 & 1 & & 3.6 & -1.8 & & & -2.0 & -86.2 \\
\hline 15 & 195 & 104254 & 1994 & 42 & 2 & & & -1.8 & & & -2.0 & -16.4 \\
\hline 15 & 195 & 104313 & 1996 & 42 & 3 & & & -1.8 & & & -2.0 & -30.2 \\
\hline 15 & 195 & 104326 & 1998 & 42 & 4 & & & -1.8 & & & -2.0 & -653.4 \\
\hline 15 & 195 & 104413 & 2000 & 42 & 5 & & & & & & -2.0 & r \\
\hline 15 & 195 & 104439 & 2002 & 42 & 6 & & & -1.8 & & & -2.1 & 21.1 \\
\hline 15 & 195 & 104451 & 2004 & 42 & 7 & & 3.8 & -1.8 & & & -2.1 & 3.9 \\
\hline 15 & 195 & 104503 & 2006 & 42 & 8 & & 2.6 & -1.8 & & & -2.1 & 1.0 \\
\hline 15 & 195 & 104516 & 2008 & 42 & 9 & & & -1.8 & & & -2.1 & 110.8 \\
\hline 15 & 195 & 104528 & 2010 & 42 & 10 & & & & & & -2.1 & 112.8 \\
\hline 15 & 195 & 104554 & 2012 & 42 & 11 & & & & & & -2.1 & 60.0 \\
\hline 15 & 195 & 104 & & 42 & 12 & & & & & & -2.1 & 23.3 \\
\hline 15 & 195 & 104622 & 16 & 42 & 13 & & & -1 & & & -2 & 0.0 \\
\hline 15 & 195 & 104637 & 18 & 42 & 4 & & .1 & -1 & & & -2.1 & -66.6 \\
\hline 15 & 195 & 10 & & 42 & 15 & & & -1.9 & & & -2.1 & 40. \\
\hline 15 & 19 & 15 & 22 & 42 & 16 & & & -1.9 & & & -2.2 & 71. \\
\hline 15 & 195 & 104729 & 24 & 42 & 17 & & & -1.9 & & & -2.2 & 23.3 \\
\hline 15 & 195 & 104743 & 2026 & 42 & 18 & & & -1 & & & -2.2 & 02.6 \\
\hline 15 & 195 & 104756 & 028 & 42 & 19 & & & -1.9 & & & -2.2 & 61.6 \\
\hline 15 & 195 & 04810 & 2030 & 42 & 20 & & & -1.9 & & & -2.2 & 44.6 \\
\hline 15 & 195 & 104823 & 2032 & 42 & 21 & & & & & & -2.2 & -189.2 \\
\hline 15 & 195 & 104836 & 2034 & 42 & 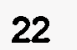 & & & & & & -2.2 & \\
\hline 15 & 19 & 48 & 036 & 42 & 23 & & & & & & -2.2 & -83.3 \\
\hline 15 & 19 & 59 & 038 & 42 & 24 & & & -2 & & & -2.2 & -70.2 \\
\hline 15 & 19 & 1 & 100 & 42 & 25 & & & -2 & & & -2.2 & -9.8 \\
\hline 15 & 19 & 25 & 2010 & 42 & 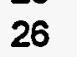 & & & -2 & & & -2.2 & 5.9 \\
\hline 15 & 195 & 104937 & 2044 & 42 & 27 & & 6.8 & -2.0 & & & -2.2 & 1.5 \\
\hline 15 & 195 & 104951 & 2046 & 42 & 28 & & & -2.0 & & & -2.3 & -7.2 \\
\hline 15 & 195 & 105003 & 2048 & 42 & 29 & & & -2.0 & & & -2.3 & -5.2 \\
\hline 15 & 195 & 105 & 2050 & 42 & 30 & & & -2 & & & -2.3 & -0 . \\
\hline 15 & 19 & & 20 & 42 & & & & -2 & & & -2.3 & 8 \\
\hline 15 & 19 & & 205 & 42 & & & & -2 & & & -2.3 & .7 \\
\hline 15 & 19 & & 2056 & 42 & & & & -2.1 & & & -2.3 & \\
\hline 15 & 195 & & 2058 & 42 & $\boldsymbol{T}$ & & 2.3 & -2.1 & & & -2.3 & 106.6 \\
\hline 15 & 195 & & 2060 & 42 & 35 & & & -2.1 & & & -2.3 & \\
\hline 15 & 195 & 105216 & 2062 & 42 & 36 & & $\$ 1.1$ & -2.1 & & & -2.3 & 093.1 \\
\hline 15 & 195 & 105250 & 2064 & 42 & 37 & & 92.9 & -2.1 & & & -2.4 & -957.1 \\
\hline 1 & 19 & & 66 & 42 & 38 & & & -2.1 & & & -2.4 & -222.6 \\
\hline 15 & 19 & & & 42 & 39 & & & -2.1 & & & -2.4 & -79.7 \\
\hline 15 & 19 & & 2070 & 42 & 40 & & & -2.1 & & & -2.4 & 50.8 \\
\hline & 195 & 105352 & 2072 & 42 & 41 & $51639 ?$ & 516413 & -2.1 & 51714.4 & 51716.8 & -2.4 & 23.3 \\
\hline
\end{tabular}


TOP FIELD (nT)

\begin{tabular}{|c|c|c|c|c|c|c|c|c|c|c|c|c|}
\hline INE & $A Y$ & & A. & & & rr.) & (Corr.) & 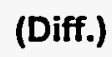 & 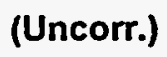 & $0^{\circ}$ & Diff.) & 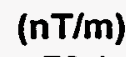 \\
\hline 15 & 195 & & 2074 & 42 & 42 & 51602.8 & 51605 & -2.2 & 56.4 & & -2.4 & 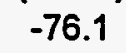 \\
\hline 15 & 19 & 10 & 2076 & 42 & 43 & 51553.8 & 51556 & -2.2 & ?51331.2 & 51333.6 & -2.4 & -364.9 \\
\hline 15 & 195 & 105439 & 2078 & 42 & 4 & 23.4 & 51625.6 & -2.2 & $? 51442$ & 444.4 & -2.4 & -297.4 \\
\hline 15 & 195 & 105452 & 2080 & 42 & 45 & 51711.0 & 713.2 & -2.2 & 672.6 & 675.0 & -2.4 & -62.9 \\
\hline 15 & 195 & 05506 & 2082 & 42 & 46 & & 716.8 & -2.2 & 51749.6 & 1752.1 & -2.5 & 7.4 \\
\hline 15 & 195 & 105520 & 084 & 42 & 47 & & 1659 & -2.2 & 1.2 & 1693.7 & -2.5 & 56.4 \\
\hline 15 & 195 & 105533 & 2086 & 42 & 48 & & 1550.6 & -2.2 & & 81.7 & -2.5 & 50.5 \\
\hline 15 & 195 & 105547 & 2088 & 42 & 49 & & 51399 & -2.2 & & 51320.7 & -2.5 & -128.9 \\
\hline 15 & 195 & 105602 & 2090 & 42 & 50 & & 51249.4 & -2.2 & $? 51$ & 39.9 & -2.5 & -343.9 \\
\hline 15 & 195 & 105616 & 092 & 42 & 51 & 22.2 & 1224.4 & -2.2 & 7.2 & 29.7 & -2.5 & -647.5 \\
\hline 15 & 195 & 105632 & 094 & 42 & 52 & 1.8 & & -2.2 & .6 & 99.1 & -2.5 & -352.8 \\
\hline 15 & 195 & 105644 & 2096 & 42 & 53 & 1.8 & 51814 & -2.2 & 5.2 & 7.7 & -2.5 & -469.8 \\
\hline 15 & 195 & 105657 & 098 & 42 & 54 & 64.8 & 52567 & -2.2 & 7.8 & 40.3 & -2.5 & -44.3 \\
\hline 15 & 195 & 105728 & 100 & 42 & 55 & 83.2 & 53585.5 & -2.3 & 31.0 & 83.5 & -2.5 & 1799.7 \\
\hline 15 & 195 & 105743 & 102 & 42 & 56 & & 54122.5 & -2.3 & & 31.2 & -2.6 & 3128.5 \\
\hline 15 & 195 & 105 & 104 & 42 & 57 & .6 & 53864.9 & -2.3 & & 04.2 & -2.6 & 2195.1 \\
\hline 15 & 195 & 105812 & 106 & 42 & 58 & .4 & 8.7 & -2.3 & $? 5$ & 48.0 & -2.6 & 342.6 \\
\hline 15 & 195 & 105826 & 108 & 42 & 59 & & 52526.9 & -2.3 & & 19.2 & -2.6 & -127.9 \\
\hline 15 & 195 & 105839 & 2110 & 42 & 60 & & 52178.9 & -2.3 & & 2.2 & -2.6 & -175.4 \\
\hline 15 & 195 & 105852 & 2112 & 42 & 61 & 51 & 51993.9 & -2.3 & & 7.8 & -2.6 & -125.3 \\
\hline 15 & 195 & 105906 & 2114 & 42 & 62 & & 51892.9 & -2.3 & & 3.8 & -2.6 & -97.4 \\
\hline 15 & 195 & 105918 & 116 & 42 & 63 & & 31.1 & -2.3 & & 3.2 & -2.6 & -79.0 \\
\hline 15 & 195 & 105931 & 118 & 42 & 64 & & 35.9 & -2.3 & & 9.2 & -2.6 & -60.7 \\
\hline 15 & 195 & 105 & 20 & 42 & 65 & & 56.9 & -2.3 & & 9.2 & -2.6 & -45.9 \\
\hline 15 & 195 & & 122 & 42 & 66 & & 51732.5 & -2.3 & & 0.8 & -2.6 & -36.1 \\
\hline 15 & 19 & & 124 & 42 & 6 & & & -2.4 & & 9.5 & -2.7 & -22.6 \\
\hline 15 & 195 & & 126 & 42 & 8 & & 5.6 & -2.4 & & 0.5 & -2.7 & -8.9 \\
\hline 15 & 195 & 43 & 128 & 42 & 69 & & 1.8 & -2.4 & & & -2.7 & 9.2 \\
\hline 15 & 195 & 1 & 130 & 42 & 70 & & 0.2 & -2.4 & & .3 & -2.7 & 8.5 \\
\hline 15 & 195 & 16 & 2132 & 42 & 71 & 2.2 & 4.6 & -2.4 & 2.6 & 5.3 & -2.7 & 6.2 \\
\hline 15 & 195 & 110140 & 2134 & 42 & 72 & & 16 & -2.4 & & 8.5 & -2.7 & 734.1 \\
\hline 15 & 195 & 110156 & 2136 & 42 & 73 & 5.4 & 17.8 & -2.4 & & & -2.7 & -342.6 \\
\hline 15 & 195 & 110213 & 2138 & 42 & 74 & & 51572.6 & -2.4 & & & -2.7 & -114.1 \\
\hline 15 & 195 & 110 & 40 & 42 & 75 & & 51637.6 & -2.4 & & 5.7 & -2.7 & -52.8 \\
\hline 15 & 19 & & 142 & 42 & 76 & & & -2.4 & & 55.1 & -2.7 & -36.4 \\
\hline 15 & 195 & & 2144 & 42 & 77 & & & -2.4 & & 7.8 & -2.8 & -26.2 \\
\hline 16 & 195 & 1 & 146 & 45 & 76 & & & -2.6 & & & -2.9 & -31.8 \\
\hline 16 & 195 & 110 & 0 & 45 & 75 & & & -2.6 & & & -2.9 & -38.4 \\
\hline 16 & 195 & 110701 & 2150 & 45 & 74 & & 27.6 & -2.6 & & & -2.9 & -117.7 \\
\hline 16 & 195 & 110715 & 2152 & 45 & 73 & 228.2 & 51230.8 & -2.6 & & 4.9 & -2.9 & -485.6 \\
\hline 16 & 195 & 110731 & 2154 & 45 & 72 & ?52228.8 & 52231.4 & -2.6 & 1.4 & 04.3 & -2.9 & 1102.6 \\
\hline 16 & 195 & 110 & 2156 & 45 & 71 & 786.6 & 50789.2 & -2.6 & 9.2 & 2.1 & -2.9 & 512.5 \\
\hline 16 & 19 & & & 45 & 70 & & 51378.6 & -2.6 & & 30.6 & -3.0 & 166.6 \\
\hline 16 & 19 & & 2700 & 45 & 69 & & & -2.6 & & 9.8 & -3.0 & 46.9 \\
\hline 16 & 195 & 110830 & 2162 & 45 & 68 & & & -2.6 & & 2.2 & -3.0 & 13.1 \\
\hline 16 & 195 & 110843 & 2164 & 45 & 67 & & 51679.5 & -2.7 & & 6.2 & -3.0 & -5.9 \\
\hline 16 & 195 & 110857 & 2166 & 45 & 66 & 51700.4 & 51703.1 & -2.7 & & 95.4 & -3.0 & -13.1 \\
\hline 16 & 195 & 110915 & 168 & 45 & 65 & 51718.6 & 21.3 & -2.7 & & 07.2 & -3.0 & -23.6 \\
\hline 16 & 195 & 110927 & 170 & 45 & 64 & 51736.6 & 51739.3 & -2.7 & 51716.0 & 51719.0 & -3.0 & -33.8 \\
\hline
\end{tabular}

BOTTOM FIELD (nT) GRAD.

nT/m)

$-76.1$

297.4

62.9

57.4

56.4

$-128.9$

$-343.9$

$-347.5$

409.8

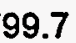

128.5

(95.1

$-127.9$

$-175.4$

$-125.3$

$-97.4$

$-60.7$

$-45.9$

36.1

8.9

8.5

66.2

$-734.1$

$-342.6$

14.1

$-52.8$

$-36.4$

$-26.2$

$-31.8$

$-38.4$

$-117.7$

485.6

102.6

12.5

166.6

46.9

13.1

$-5.9$

$-23.6$

$-33.8$ 
TOP FIELD (nT)

LINE DAY TIME STA. $X \quad Y$ (Uncorr.) (Corr.)

$\begin{array}{llllll}16 & 195 & 110939 & 2172 & 45 & 63\end{array}$

$\begin{array}{llllll}16 & 195 & 110953 & 2174 & 45 & 62\end{array}$

$\begin{array}{llllll}16 & 195 & 111006 & 2176 & 45 & 61\end{array}$

$\begin{array}{llllll}16 & 195 & 111019 & 2178 & 45 & 60\end{array}$

$\begin{array}{llllll}16 & 195 & 111031 & 2180 & 45 & 59\end{array}$

$\begin{array}{llllll}16 & 195 & 111043 & 2182 & 45 & 58\end{array}$

$\begin{array}{llllll}16 & 195 & 111054 & 2184 & 45 & 57\end{array}$

$\begin{array}{lllllll}16 & 195 & 111106 & 2186 & 45 & 56\end{array}$

$\begin{array}{lllllll}16 & 195 & 111119 & 2188 & 45 & 55 & ? 52\end{array}$

$\begin{array}{lllllll}16 & 195 & 111134 & 2190 & 45 & 54 & 51900.8\end{array}$

$\begin{array}{lllllll}16 & 195 & 111148 & 2192 & 45 & 53 & 51665.6\end{array}$

$\begin{array}{lllllll}16 & 195 & 111200 & 2194 & 45 & 52 & 51489.8\end{array}$

$\begin{array}{lllllll}16 & 195 & 111212 & 2196 & 45 & 51 & 51435.0\end{array}$

$\begin{array}{lllllll}16 & 195 & 111223 & 2198 & 45 & 50 & 51491.2\end{array}$

$\begin{array}{lllllll}16 & 195 & 111238 & 2200 & 45 & 49 & 51581.4\end{array}$

$\begin{array}{lllllll}16 & 195 & 111251 & 2202 & 45 & 48 & 51703.4\end{array}$

$\begin{array}{lllllll}16 & 195 & 111303 & 2204 & 45 & 47 & 51833.2\end{array}$

$\begin{array}{lllllll}16 & 195 & 111317 & 2206 & 45 & 46 & 51960.0\end{array}$

$\begin{array}{lllllll}16 & 195 & 111332 & 2208 & 45 & 45 & 51997.8\end{array}$

$\begin{array}{lllllll}16 & 195 & 111349 & 2210 & 45 & 44 & 51859.8\end{array}$

$\begin{array}{lllllll}16 & 195 & 111408 & 2212 & 45 & 43 & 51699.4\end{array}$

$\begin{array}{lllllll}16 & 195 & 111420 & 2214 & 45 & 42 & 51645.8\end{array}$

$\begin{array}{lllllll}16 & 195 & 111433 & 2216 & 45 & 41 & 51676.2\end{array}$

$\begin{array}{lllllll}16 & 195 & 111444 & 2218 & 45 & 40 & 51714.4\end{array}$

$\begin{array}{lllllll}16 & 195 & 111457 & 2220 & 45 & 39 & 51834.4\end{array}$

$\begin{array}{lllllll}16 & 195 & 111509 & 2222 & 45 & 38 & 252167.8\end{array}$

$\begin{array}{lllllll}16 & 195 & 111526 & 2224 & 45 & 37 & ? 52704.4\end{array}$

$\begin{array}{lllllll}16 & 195 & 111539 & 2226 & 45 & 36 & ? 52528.6\end{array}$

$\begin{array}{lllll}16 & 195 & 111559 & 2228\end{array}$

$\begin{array}{llll}16 & 195 & 111620 & 2230\end{array}$

$\begin{array}{llll}16 & 195 & 111633 & 2232\end{array}$

$\begin{array}{llll}16 & 195 & 111645 \quad 2234\end{array}$

$\begin{array}{llll}16 & 195 & 111658 & 2236\end{array}$

$\begin{array}{llll}16 & 195 & 111710 & 2238\end{array}$

$\begin{array}{llll}16 & 195 & 111723 \quad 2240\end{array}$

$16 \quad 195 \quad 111736 \quad 2242$

$16 \quad 195 \quad 111749 \quad 2244$

$\begin{array}{llll}16 & 195 & 111803 \quad 2246\end{array}$

$16 \quad 195 \quad 111816 \quad 2248$

$16 \quad 195 \quad 111828 \quad 2250$

$\begin{array}{lllll}16 & 195 & 111840 & 2252\end{array}$

$\begin{array}{llllll}16 & 195 & 111853 & 2254 & 45 & 22\end{array}$

$\begin{array}{lllllll}16 & 195 & 111909 & 2256 & 45 & 21 & 253623.2\end{array}$

$\begin{array}{lllllll}16 & 195 & 111926 & 2258 & 45 & 20 & ? 58584\end{array}$

$\begin{array}{lllllll}16 & 195 & 111947 & 2260 & 45 & 19 & ? 55490.8\end{array}$

$\begin{array}{lllllll}16 & 195 & 112002 & 2262 & 45 & 18 & ? 58545.6\end{array}$

$\begin{array}{lllllll}16 & 195 & 112016 & 2264 & 45 & 17 & ? 52956.8\end{array}$

$\begin{array}{lllllll}16 & 195 & 112028 & 2266 & 45 & 16 & ? 51873.6\end{array}$

$\begin{array}{lllllll}16 & 195 & 112052 & 2268 & 45 & 15 & ? 51446.4\end{array}$
BOTTOM FIELD (nT)

GRAD.

(Diff.) (Uncorr.) (Corr.) (Diff.) (nT/m)

$51788.5-2.7$

$51820.7-2.7$

$51861.1-2.7$

$51930.1-2.7$

$\begin{array}{lll}52016.9 & -2.7\end{array}$

$52102.5 \quad-2.7$

$52130.5 \quad-2.7$

$52062.3 \quad-2.7$

$51903.5-2.7$

$51668.4 \quad-2.8$

$51492.6 \quad-2.8$

$51437.8 \quad-2.8$

$51494-2.8$

$51584.2 \quad-2.8$

$\begin{array}{lll}51706.2 & -2.8\end{array}$

$51836-2.8$

$51962.8-2.8$

$52000.6-2.8$

$51862.6-2.8$

$51702.2-2.8$

$51648.6-2.8$

$51679.1-2.9$

$51717.3-2.9$

$51837.3-2.9$

$\begin{array}{lll}52170.7 & -2.9\end{array}$

$52707.3-2.9$

$\begin{array}{lll}52531.5 & -2.9\end{array}$

$51633.9-2.9$

$51445.3-2.9$

$51499.7-2.9$

$51570.9-2.9$

$51633.3-2.9$

$51672.9-2.9$

$51715.1-2.9$

$51753-3.0$

51760.6

51763.6

$-3.0$

$-41.3$

51795.4

51798.4

$-3.0$

$-37.0$

51784.8

51787.8

51841.8

51844.9

$-3.0$

$-120.7$

51911.2

51914.3

$-3.1$

$-140.3$

?51982.2

51985.3

$-3.1$

$-168.9$

$? 51996$

51999.1

$? 51909$

51912.1

?51743.2

51746.3

?51518.4

?51304.8

51521.5

51307.9

51302.0

51305.1

51416.8

51419.9

51563.4

51566.5

51696.6

51699.7

51863.0

52073.4

51866.1

$? 52201$

52076.6

?51881.8

52204.2

?51582.8

51885.0

$-3.1$

$-192.8$

51629.2

51586.0

51729.4

51632.4

$-3.1$

$-216.1$

$-3.1 \quad-246.9$

$-3.1 \quad-258.4$

$-3.1 \quad-241.3$

$-3.1 \quad-303.3$

$-3.1-218.0$

51730.2

51732.6

$-122.0$

51923.0

51733.4

51926.2

?52551.4

52554.6

?58589.4

58592.6

?58610

58613.2

?51142.2

51145.5

?51269

51272.3

51453.0

51456.3

51562.2

51565.5

51634.0

51637.3

51677.0

51680.3

$-3.1$

$-29.5$

$-3.1 \quad-11.1$

51714.2

51717.5

$-3.1$

48.9

$-3.2$

185.9

333.1

36.1

$-191.2$

$-3.2$

$-27.2$

$-3.2 \quad 87.2$

$-3.2$

25.9

51746.4

51749.7

$-3.2$

145.2

$51779.6-3.0$

51762.0

51765.3

628.9

9647.5

9969.5

$-3.3 \quad-801.3$

$-3.3 \quad-284.3$

$51803.6-3.0$

51706.6

51709.9

$-3.3$

$-71.8$

$51887.2-3.0$

51767.0

51770.4

$-3.3$

$-9.5$

$52075.2-3.0$

52046.6

52050.0

$-3.3$

5.9

52304.8

52308.2

$-3.3$

11.5

$-3.3 \quad 3.3$

$52348.6-3.0$

$? 52814$

52817.4

$-3.3$

$-5.9$

$52809.4-3.0$

$? 53876$

53879.4

$-3.3$

$-23.9$

?58568

58571.4

$-3.3$

$-154.1$

$-192.1$

$-3.4 \quad-42.0$

$58587-3.0$

$55493.8-3.0$

?58586.8

58590.2

$-3.4$

$-66.9$

12.5

$58548.6-3.0$

$? 58566$

58569.4

?52523.4

52526.8

414.4

$-26.2$

5075.4

$52959.8-3.0$

?51320

51323.4

$51449.5 \quad-3.1$

?51172.8

51176.3

$-3.4$

$-3.4$

$-3.4$

33.4

$-710.5$

$-3.4 \quad-907.5$

$-3.5 \quad-448.5$ 
TOP FIELD (nT)

LINE DAY TIME STA. $X \quad Y$ (Uncorr.) (Corr.)

$\begin{array}{lllllll}16 & 195 & 112104 & 2270 & 45 & 14 & 51349.6\end{array}$

$\begin{array}{lllllll}16 & 195 & 112116 & 2272 & 45 & 13 & 51395.2\end{array}$

$\begin{array}{lllllll}16 & 195 & 112128 & 2274 & 45 & 12 & 51470.8\end{array}$

$\begin{array}{lllllll}16 & 195 & 112138 & 2276 & 45 & 11 & 51541.6\end{array}$

$\begin{array}{lllllll}16 & 195 & 112150 & 2278 & 45 & 10 & 51595.8\end{array}$

$\begin{array}{lllllll}16 & 195 & 112201 & 2280 & 45 & 9 & 51620.2\end{array}$

$\begin{array}{llllll}16 & 195 & 112212 & 2282 & 45 & 8\end{array}$

$\begin{array}{llllll}16 & 195 & 112223 & 2284 & 45 & 7\end{array}$

$\begin{array}{llllll}16 & 195 & 112234 & 2286 & 45 & 6\end{array}$

$\begin{array}{llllll}16 & 195 & 112246 & 2288 & 45 & 5\end{array}$

$\begin{array}{llllll}16 & 195 & 112257 & 2290 & 45 & 4\end{array}$

$\begin{array}{llllll}16 & 195 & 112308 & 2292 & 45 & 3\end{array}$

$\begin{array}{llllll}16 & 195 & 112319 & 2294 & 45 & 2\end{array}$

$\begin{array}{llllll}16 & 195 & 112330 & 2296 & 45 & 1\end{array}$

$\begin{array}{lllll}16 & 195 & 112342 & 2298 & 45\end{array}$

$\begin{array}{lllll}17 & 195 & 124115 & 2302 & 48\end{array}$

$\begin{array}{llllll}17 & 195 & 124144 & 2304 & 48\end{array}$

$\begin{array}{llllll}17 & 195 & 124209 & 2306 & 48 & 2\end{array}$

$\begin{array}{llllll}17 & 195 & 124228 & 2308 & 48 & 3\end{array}$

$\begin{array}{llllll}17 & 195 & 124306 & 2310 & 48 & 4\end{array}$

$\begin{array}{llllll}17 & 195 & 124325 & 2312 & 48 & 5\end{array}$

$\begin{array}{llllll}17 & 195 & 124341 & 2314 & 48 & 6\end{array}$

$\begin{array}{llllll}17 & 195 & 124358 & 2316 & 48 & 7\end{array}$

$\begin{array}{llllll}17 & 195 & 124414 & 2318 & 48 & 8\end{array}$

$\begin{array}{llllll}17 & 195 & 124431 & 2320 & 48 & 9\end{array}$

$\begin{array}{llllll}17 & 195 & 124448 & 2322 & 48 & 10\end{array}$

$\begin{array}{llllll}17 & 195 & 124504 & 2324 & 48 & 11\end{array}$

$\begin{array}{llllll}17 & 195 & 124519 & 2326 & 48 & 12\end{array}$

$\begin{array}{llllll}17 & 195 & 124536 & 2328 & 48 & 13\end{array}$

$\begin{array}{llllll}17 & 195 & 124552 & 2330 & 48 & 14\end{array}$

$\begin{array}{llllll}17 & 195 & 124607 & 2332 & 48 & 15\end{array}$

$\begin{array}{llllll}17 & 195 & 124624 & 2334 & 48 & 16\end{array}$

$\begin{array}{llllll}17 & 195 & 124642 & 2336 & 48 & 17\end{array}$

$\begin{array}{llllll}17 & 195 & 124701 & 2338 & 48 & 18\end{array}$

$\begin{array}{llllll}17 & 195 & 124718 & 2340 & 48 & 19\end{array}$

$\begin{array}{llllll}17 & 195 & 124734 & 2342 & 48 & 20\end{array}$

$\begin{array}{llllll}17 & 195 & 124751 & 2344 & 48 & 21\end{array}$

$\begin{array}{llllll}17 & 195 & 124808 & 2346 & 48 & 22\end{array}$

$\begin{array}{llllll}17 & 195 & 124825 & 2348 & 48 & 23\end{array}$

$\begin{array}{llllll}17 & 195 & 124840 & 2350 & 48 & 24\end{array}$

$\begin{array}{llllll}17 & 195 & 124853 & 2352 & 48 & 25\end{array}$

$\begin{array}{llllll}17 & 195 & 124908 & 2354 & 48 & 26\end{array}$

$\begin{array}{llllll}17 & 195 & 124929 & 2356 & 48 & 27\end{array}$

$\begin{array}{llllll}17 & 195 & 124946 & 2358 & 48 & 28\end{array}$

$\begin{array}{llllll}17 & 195 & 125001 & 2360 & 48 & 29\end{array}$

$\begin{array}{llllll}17 & 195 & 125018 & 2362 & 48 & 30\end{array}$

$\begin{array}{llllll}17 & 195 & 125034 & 2364 & 48 & 31\end{array}$

$\begin{array}{llllll}17 & 195 & 125050 & 2366 & 48 & 32\end{array}$

$\begin{array}{llllll}17 & 195 & 125110 & 2368 & 48 & 33\end{array}$
51638.6

51651.6

51647.6

51629.4

51610.8

51606.2

51594.4

51550.8

251443.4

51440.6

51550.2

51596.2

51622.0

51635.2

51641.6

51644.4

51641.2

51636.8

51628.6

51615.0

51601.6

51591.6

51594.4

51623.0

51716.2

51917.8

$? 52274$

$? 53019$

253652.8

?53642.8

$? 53082.2$

52572.0

52260.0

52054.2

51933.8

51858.4

51795.0

51749.2

51661.4

51619.2

51573.8

51525.8
51704.4 $\begin{array}{lll}51352.7 & -3.1 & 51228.6\end{array}$

$51398.3-3.1$

$51473.9-3.1$

$51544.7-3.1$

$51598.9-3.1$

$51623.3-3.1$

$51641.7-3.1$

$51654.7-3.1$

$51650.7 \quad-3.1$

$51632.5 \quad-3.1$

$51613.9-3.1$

$51609.3-3.1$

$51597.6-3.2$

$51554-3.2$

$\begin{array}{lll}51446.6 & -3.2\end{array}$

$\begin{array}{ll}51452 & -11.4\end{array}$

$51561.6-11.4$

$51607.7-11.5$

$51633.5-11.5$

$51646.8-11.6$

$51653.2-11.6$

$51656 \quad-11.6$

$51652.9-11.7$

$51648.5-11.7$

$51640.3-11.7$

$\begin{array}{lll}51626.8 & -11.8\end{array}$

$51613.4-11.8$

$51603.4-11.8$

$51606.2-11.8$

$51634.9-11.9$

$51728.1-11.9$

$\begin{array}{lll}51929.7 & -11.9\end{array}$

$52286-12.0$

$53031-12.0$

$53664.8-12.0$

$\begin{array}{ll}53654.9 & -12.1\end{array}$

$53094.3-12.1$

$52584.1-12.1$

$52272.1-12.1$

$52066.4-12.2$

$51946-12.2$

$51870.6-12.2$

$51807.3-12.3$

$\begin{array}{lll}51761.5 & -12.3\end{array}$

$51716.7-12.3$

$51673.7-12.3$

$51631.6-12.4$

$51586.2-12.4$

$51538.2-12.4$

51464.6

51550.4

51600.6

51618.8

51645.4

51656.0

51649.4

51622.2

51603.6

51600.4

51576.2

51499.6

51290.0

51353.2

51503.0

51569.0

51602.0

51619.2

51629.0

51632.8

51630.2

51618.0

51618.0

51596.8

51577.8

51554.6

51537.8

51520.6

51561.2

?51690.2

$? 52877.6$

?53806.8

?53937.6

?53121.8

52531.4

52216.2

52019.2

51913.8

51844.2

51788.4

51740.2

51699.0

51657.2

51609.8

51553.4

51482.6

BOTTOM FIELD (nT)

GRAD.

$\begin{array}{llll}512321 & -3.5 & -198.4\end{array}$

$\begin{array}{llll}51359.2 & 51362.7 & -3.5 & -59.0\end{array}$

51468.1

$-3.5$

$-10.2$

51553.9

$-3.5$

14.4

.

$-3.5$

7.9

$-3.5$

$-2.3$

$-3.5$

11.1

$-3.5$

7.2

51625

$-3.5$

3.0

51607.1

$-3.5$

$-11.8$

51603.9

51579.7

51503.2

51293.6

$-3.5$

$-11.8$

51361.8

51511.7

$-3.5$

$-9.5$

51577.7

$-3.5$

$-29.8$

51610.7

$-3.6$

$-83.9$

51628.0

$-3.6$

$-251.5$

51637.8

51641.6

51639.0

51626.8

51626.9

51605.7

51586.7

51563.5

51546.7

51529.6

51570.2

51699.2

52031.4

52886.6

53815.9

53946.7

$-8.7$

$-143.3$

$-8.7 \quad-44.6$

$-8.7-32.8$

$\begin{array}{ll}-8.8 & -26.2\end{array}$

$\begin{array}{ll}-8.8 & -20.7\end{array}$

$-8.8 \quad-19.0$

$-8.8-18.0$

$-8.8-30.8$

$-8.9 \quad-17.4$

$-8.9 \quad-29.8$

$-8.9 \quad-39.0$

$-8.9 \quad-60.7$

$-8.9 \quad-92.8$

$-9.0 \quad-167.9$

$-9.0 \quad-254.1$

$-9.0 \quad-373.1$

$-9.0 \quad-412.5$

$-9.0 \quad-231.8$

53130.9

$-9.1 \quad 252.5$

52540.5

$-9.1 \quad 483.3$

52225.3

$-9.1$

64.9

52028.3

$-66.6$

51923

$-71.8$

51853.4

51797.6

51749.4

51708.2 
TOP FIELD (nT)

LINE DAY TIME STA. $X \quad Y$

$\begin{array}{llllll}17 & 195 & 125125 & 2370 & 48 & 34\end{array}$

$\begin{array}{llllll}17 & 195 & 125141 & 2372 & 48 & 35\end{array}$

$\begin{array}{llllll}17 & 195 & 125200 & 2374 & 48 & 36\end{array}$

$\begin{array}{llllll}17 & 195 & 125221 & 2376 & 48 & 37\end{array}$

$\begin{array}{llllll}17 & 195 & 125241 & 2378 & 48 & 38\end{array}$

$\begin{array}{lllllll}17 & 195 & 125257 & 2380 & 48 & 39\end{array}$

$\begin{array}{llllll}17 & 195 & 125312 & 2382 & 48 & 40\end{array}$

$\begin{array}{llllll}17 & 195 & 125326 & 2384 & 48 & 41\end{array}$

$\begin{array}{llllll}17 & 195 & 125342 & 2386 & 48 & 42\end{array}$

$\begin{array}{llllll}17 & 195 & 125400 & 2388 & 48 & 43\end{array}$

$\begin{array}{llllll}17 & 195 & 125421 & 2390 & 48 & 44\end{array}$

$\begin{array}{llllll}17 & 195 & 125444 & 2392 & 48 & 45\end{array}$

$\begin{array}{llllll}17 & 195 & 125510 & 2394 & 48 & 46\end{array}$

$\begin{array}{llllll}17 & 195 & 125525 & 2396 & 48 & 47\end{array}$

$\begin{array}{llllll}17 & 195 & 125539 & 2398 & 48 & 48\end{array}$

$\begin{array}{llllll}17 & 195 & 125559 & 2400 & 48 & 49\end{array}$

$\begin{array}{llllll}17 & 195 & 125618 & 2402 & 48 & 50\end{array}$

$\begin{array}{llllll}17 & 195 & 125633 & 2404 & 48 & 51\end{array}$

$17 \quad 1951256522406$

$\begin{array}{lllll}17 & 195 & 125711 & 2408\end{array}$

$\begin{array}{lllll}17 & 195 & 125726 & 2410\end{array}$

$\begin{array}{llll}17 & 195 & 125742 & 2412\end{array}$

$\begin{array}{llll}17 & 195 & 125800 & 2414\end{array}$

$\begin{array}{lllll}17 & 195 & 125815 & 2416\end{array}$

$\begin{array}{llll}17 & 195 & 125829 & 2418\end{array}$

$\begin{array}{lllll}17 & 195 & 125848 & 2420\end{array}$

$\begin{array}{lllll}17 & 195 & 125903 & 2422\end{array}$

$\begin{array}{lllll}17 & 195 & 125921 & 2424\end{array}$

$\begin{array}{lllll}17 & 195 & 125935 & 2426\end{array}$

$\begin{array}{lllll}17 & 195 & 125949 & 2428\end{array}$

$\begin{array}{lllll}17 & 195 & 130004 & 2430\end{array}$

$\begin{array}{llll}17 & 195 & 130018 & 2432\end{array}$

$\begin{array}{lllll}17 & 195 & 130034 & 2434\end{array}$

$\begin{array}{lllll}17 & 195 & 130049 & 2436\end{array}$

$\begin{array}{lllll}17 & 195 & 130104 & 2438\end{array}$

$\begin{array}{llllll}17 & 195 & 130118 & 2440 & 48 & 69\end{array}$

$\begin{array}{llllll}17 & 195 & 130133 & 2442 & 48 & 70\end{array}$

$\begin{array}{lllll}17 & 195 & 130148 & 2444\end{array}$

$\begin{array}{lllll}17 & 195 & 130206 & 2446\end{array}$

$\begin{array}{lllll}17 & 195 & 130224 & 2448\end{array}$

$\begin{array}{lllllll}17 & 195 & 130240 & 2450 & 48 & 74 & 51537.0\end{array}$

$\begin{array}{lllllll}17 & 195 & 130255 & 2452 & 48 & 75 & 51611.0\end{array}$

$\begin{array}{lllllll}18 & 195 & 130850 & 2454 & 51 & 77 & 51668.6\end{array}$

$\begin{array}{lllllll}18 & 195 & 130906 & 2456 & 51 & 76 & 51634.8\end{array}$

$\begin{array}{lllllll}18 & 195 & 130923 & 2458 & 51 & 75 & 51552.6\end{array}$

$\begin{array}{lllllll}18 & 195 & 130940 & 2460 & 51 & 74 & ? 51447\end{array}$

$\begin{array}{lllllll}18 & 195 & 130959 & 2462 & 51 & 73 & ? 51326.4\end{array}$

$\begin{array}{lllllll}18 & 195 & 131030 & 2464 & 51 & 72 & ? 51461\end{array}$

$\begin{array}{lllllll}18 & 195 & 131052 & 2466 & 51 & 71 & ? 50899\end{array}$
BOTTOM FIELD (nT)

GRAD.

(Corr.)

(Diff.) $51503.9-12.5$

$51526.9-12.5$

$51654.5-12.5$

$51649.4-12.6$

$51570.2-12.6$

$51522-12.6$

$51502.1-12.7$

$51454.9-12.7$

$51410.9-12.7$

$51487.5-12.7$

$51765.8-12.8$

$\begin{array}{lll}52020.6 & -12.8\end{array}$

$52098.9-12.9$

$52056.9-12.9$

$51915.9-12.9$

$51707.8-13.0$

$51480.6-13.0$

$51246.4-13.0$

$51100.8-13.0$

$51019.9-13.1$

$51026.9-13.1$

$51284.9-13.1$

$51678.6-13.2$

$51874.4-13.2$

$51857.4-13.2$

$51805.1-13.3$

$51771.5-13.3$

$51753.1-13.3$

$51740.5-13.3$

$51730.8-13.4$

$51721.6-13.4$

$\begin{array}{lll}51710 & -13.4\end{array}$

$51697.8-13.4$

$51677.1-13.5$

$51646.3-13.5$

$51581.3-13.5$

$51420.3-13.5$

$50891.4-13.6$

$49858.6-13.6$

$51313.4-13.6$

$51550.7-13.7$

$51624.7-13.7$

$51682.9-14.3$

$51649.2-14.4$

$\begin{array}{lll}51567 & -14.4\end{array}$

$51461.4-14.4$

$51340.9-14.5$

$51475.5-14.5$

$50913.5-14.5$
(Uncorr.)

51395.6

?51297.4

?51741.8

51561.8

51419.4

51411.2

51426.6

51371.4

?51170.6

251164

?51791.6

?52240.6

?52307.4

?52257.2

52049.6

51754.2

51433.8

51062.2

?50828.4

?50696.4

?50516.2

?50874

?51592.8

?51804.2

51725.8

51674.0

51681.4

51678.2

51677.8

51676.6

51674.2

51671.0

51661.6

51650.2

51627.6

51579.6

51482.2

$? 51236$

?50549.8

51240.8

51494.2

51586.0

51606.6

51524.8

51398.8

51293.0

$? 50826$

?50339.6

?51068.8
(Corr.)

(Diff.)

$51404.9 \quad-9.3$

$-9.3$

$51751.2-9.4$

51571.2

51428.8

51420.6

51436.0

51380.9

51180.1

51173.5

51801.1

52250.1

52317.0

52266.8

52059.2

51763.8

51443.4

51071.9

50838.1

50706.1

50525.9

50883.7

51602.6

51814.0

51735.6

51683.8

51691.2

51688.0

51687.7

51686.5

51684.1

51680.9

51671.5

51660.1

51637.6

51589.6

51492.2

51246.0

50559.8

$-9.4$

$-9.4$

$-9.4$

$-9.4$

$-9.5$

$-9.5$

$-9.5$

$-9.5$

$-9.5$

$-9.6$

$-9.6$

$-9.6$

$-9.6$

$-9.6$

$-9.7$

$-9.7$

$-9.7$

$-9.7$

$-9.7$

$-9.8$

$-9.8$

$-9.8$

$-9.8$

$-9.8$

$-9.8$

$-9.9$

$-9.9$

$-9.9$

$-9.9$

$-9.9$

$-9.9$

$-10.0$

$-10.0$

$-10.0$

$-10.0$

51250.8

$-10.0$

51504.3

$-10.0$

51596.1

$-10.1$

$-10.1$

$51617.1-10.5$

$51535.3-10.5$

$51409.3 \quad-10.5$

$51303.5 \quad-10.5$

$50836.5-10.5$

$50350.2-10.6$

$51079.4-10$.
(nT/m)

$-157.1$

$-355.7$

163.6

$-123.0$

$-226.6$

$-161.0$

$-103.0$

$-116.1$

$-373.1$

$-509.5$

63.3

381.6

363.0

349.5

240.3

97.4

$-55.4$

$-280.7$

$-425.2$

$-508.9$

$-815.7$

$-652.1$

$-119.0$

$-93.4$

$-194.1$

$-193.1$

$-125.9$

$-101.0$

$-81.0$

$-66.9$

$-55.7$

$-42.0$

$-37.4$

$-22.0$

$-8.5$

19.3

123.6

587.2

1155.4

$-96.7$

$-70.2$

$-41.0$

$-101.6$

$-180.3$ 
TOP FIELD (nT)

\begin{tabular}{|c|c|c|c|c|c|c|}
\hline INF & $A Y$ & TIME & STA. & $\mathbf{x}$ & & \\
\hline 18 & 195 & 131108 & 2468 & 51 & 70 & 51355.8 \\
\hline 18 & 195 & 131124 & 2470 & 51 & 69 & 51556.6 \\
\hline 18 & 195 & 131140 & 2472 & 51 & 68 & 51627.6 \\
\hline 18 & 195 & 131156 & 2474 & 51 & 67 & 51662.0 \\
\hline 18 & 195 & 131211 & 2476 & 51 & 66 & 51683.2 \\
\hline 18 & 195 & 131226 & 2478 & 51 & 65 & 51698.4 \\
\hline 18 & 195 & 131243 & 2480 & 51 & 64 & 51712.4 \\
\hline 18 & 195 & 131259 & 2482 & 51 & 63 & 51732.2 \\
\hline 18 & 195 & 131316 & 2484 & 51 & 62 & 51767.8 \\
\hline 18 & 195 & 131332 & 2486 & 51 & 61 & 51821.6 \\
\hline 18 & 195 & 131351 & 2488 & 51 & 60 & 51960.2 \\
\hline 18 & 195 & 131406 & 2490 & 51 & 59 & 52228.2 \\
\hline 18 & 195 & 131422 & 2492 & 51 & 58 & P52608.4 \\
\hline 18 & 195 & 131437 & 2494 & 51 & 57 & ?52627.8 \\
\hline 18 & 195 & 131452 & 2496 & 51 & 56 & ?52139.8 \\
\hline 18 & 195 & 131511 & 2498 & 51 & 55 & ?51127.6 \\
\hline 18 & 195 & 131534 & 2500 & 51 & 54 & $? 50644$ \\
\hline 18 & 195 & 131550 & 2502 & 51 & 53 & ?50549.6 \\
\hline 18 & 195 & 131605 & 2504 & 51 & 52 & $? 50688$ \\
\hline 18 & 195 & 131623 & 2506 & 51 & 51 & ?50961.6 \\
\hline 18 & 195 & 131639 & 2508 & 51 & 50 & 51327.2 \\
\hline 18 & 195 & 131654 & 2510 & 51 & 49 & 51690.8 \\
\hline 18 & 195 & 131708 & 2512 & 51 & 48 & 51999.0 \\
\hline 18 & 195 & 131727 & 2514 & 51 & 47 & ?52207 \\
\hline 18 & 195 & 131741 & 2516 & 51 & 46 & ?52230.8 \\
\hline 18 & 195 & 131756 & 2518 & 51 & 45 & 52036.4 \\
\hline 18 & 195 & 131812 & 2520 & 51 & 44 & 51704.0 \\
\hline 18 & 195 & 131828 & 2522 & 51 & 43 & 51442.2 \\
\hline 18 & 195 & 131843 & 2524 & 51 & 42 & 51365.8 \\
\hline 18 & 195 & 131901 & 2526 & 51 & 41 & 51380.8 \\
\hline 18 & 195 & 131916 & 2528 & 51 & 40 & 51391.6 \\
\hline 18 & 195 & 131931 & 2530 & 51 & 39 & 51411.6 \\
\hline 18 & 195 & 131946 & 2532 & 51 & 38 & 51500.2 \\
\hline 18 & 195 & 132003 & 2534 & 51 & 37 & 51659.0 \\
\hline 18 & 95 & 132017 & 2536 & 51 & 36 & 51629.8 \\
\hline 18 & 195 & 132033 & 2538 & 51 & 35 & 51482.4 \\
\hline 18 & 195 & 132048 & 2540 & 51 & 34 & 51475.0 \\
\hline 18 & 195 & 132102 & 2542 & 51 & 33 & 51522.0 \\
\hline 18 & 195 & 132117 & 2544 & 51 & 32 & 51571.4 \\
\hline 18 & 195 & 132131 & 2546 & 51 & 31 & 51614.6 \\
\hline 18 & 195 & 132146 & 2548 & 51 & 30 & 51653.2 \\
\hline 18 & 195 & 132202 & 2550 & 51 & 29 & 51689.4 \\
\hline 18 & 195 & 132217 & 2552 & 51 & 28 & 51723.2 \\
\hline 18 & 195 & 132233 & 2554 & 51 & 27 & 51760.6 \\
\hline 18 & 195 & 132248 & 2556 & 51 & 26 & 51797.6 \\
\hline 18 & 195 & 132303 & 2558 & 51 & 25 & 51832.8 \\
\hline 18 & 195 & 132317 & 2560 & $j 1$ & 24 & 51878.2 \\
\hline 18 & 19 & 13233 & 2562 & & 23 & 51932.6 \\
\hline 18 & & 1323 & 2564 & ग1 & 22 & 51966.6 \\
\hline
\end{tabular}

BOTTOM FIELD (nT)

(Corr.) (Diff.) (Uncorr.) (Corr.) (Diff.) $\begin{array}{lllll}51370.4 & -14.6 & 51425.4 & 51436.0 & -10.6\end{array}$ $51571.2-14.6$ $51642.2-14.6$ 51566.8

$51676.7-14.7$

51625.4

$51577.4-10.6$

51654.0

$51636.1-10.7$

$51664.7-10.7$

$51697.9-14.7$

51671.0

$51713.1-14.7$

$51727.1-14.7$

51679.0

51685.2

$\begin{array}{ll}51747 & -14.8\end{array}$

51694.4

$51782.6-14.8$

$51836.4-14.8$

51710.4

51742.6

$51975.1-14.9$

$52243.1-14.9$

51856.8

?52163.2

$52623.3-14.9$

$\begin{array}{lll}52642.7 & -14.9\end{array}$

?52959.

?53764.8

$? 52226$

- 15.0

?50300.8

$50659-15.0$

?49670

$50564.7-15.1$

$50703.1-15.1$

$50976.7-15.1$

$51342.4-15.2$

$? 50100$

?50223.8

?50621.2

51245.4

51744.8

$52014.2-15.2$

52191.8

?52518.4

?52587.2

$52246.1-15.3$

$52051.7-15.3$

?52298.6

?51651

$51457.6-15.4$

$51381.2-15.4$

?51198.8

?51180.2

$51396.2-15.4$

51271.8

$\begin{array}{ll}51407 & -15.4\end{array}$

$51427.1-15.5$

$51515.7-15.5$

$51674.5-15.5$

$51645.4-15.6$

$51498-15.6$

$51490.6-15.6$

$51537.6-15.6$

$51587.1-15.7$

$51630.3-15.7$

$51668.9-15.7$

$51705.1-15.7$

$\begin{array}{ll}51739 & -15.8\end{array}$

$51776.4-15.8$

$51813.4-15.8$

$51848.6-15.8$

$51894.1-15.9$

$51948.5-15.9$

$51982.5-15.9$
51279.6

51291.0

51455.2

?51970.8

?51855

?51358.2

51426.4

51511.2

51573.8

51625.0

51664.2

51699.0

51731.0

51772.6

51812.4

51819.6

51877.4

51914.6

51943.4
$51681.7-10.7$

$51689.7-10.7$

51705.1

$51753.4 \quad-10.8 \quad-129.5$

$\begin{array}{lll}51867.6 & -10.8 & -169.5\end{array}$

$52174.0 \quad-10.8 \quad-106.6$

$52969.8 \quad-10.8 \quad 574.8$

$\begin{array}{lll}53775.7 & -10.9 & 1863.9\end{array}$

$52236.9 \quad-10.9 \quad 141.3$

$50311.7 \quad-10.9 \quad-1355.4$

$49680.9 \quad-10.9 \quad-1596.7$

$50110.9 \quad-10.9 \quad-737.1$

$50234.8 \quad-11.0 \quad-761.0$

$50632.2 \quad-11.0 \quad-558.0$

$51256.4 \quad-11.0 \quad-134.1$

$51755.8 \quad-11.0 \quad 88.5$

$52202.8-11.0 \quad 316.1$

$52529.4-11.0 \quad 510.5$

$52598.3-11.1 \quad 584.3$

$52309.7 \quad-11.1 \quad 429.8$

$51662.1-11.1 \quad-86.9$

$51209.9 \quad-11.1 \quad-399.0$

$51191.3 \quad-11.1 \quad-304.3$

$51282.9 \quad-11.1 \quad-178.7$

$51290.8 \quad-11.2 \quad-183.6$

$51302.2 \quad-11.2 \quad-197.7$

$\begin{array}{lll}51466.4 & -11.2 & -73.8\end{array}$

$51982.0-11.2 \quad 511.1$

$51866.2 \quad-11.2 \quad 369.2$

$\begin{array}{lll}51369.4 & -11.2 & -203.6\end{array}$

$\begin{array}{lll}51437.7 & -11.3 & -79.7\end{array}$

$51522.5 \quad-11.3 \quad-17.7$

$51585.1 \quad-11.3 \quad 3.9$

$51636.3 \quad-11.3 \quad 17.0$

$\begin{array}{lll}51675.5 & -11.3 & 18.0\end{array}$

$51710.3 \quad-11.3 \quad 15.7$

$\begin{array}{lll}51742.4 & -11.4 & 12.8\end{array}$

$\begin{array}{lll}51784.0 & -11.4 & 19.7\end{array}$

$51823.8 \quad-11.4 \quad 24.3$

$\begin{array}{lll}51831.0 & -11.4 & -21.6\end{array}$

$51888.8-11.4 \quad-1.3$

$51926.0-11.4 \quad-29.5$

$\begin{array}{lll}51954.9 & -11.5 & -38.0\end{array}$ 
TOP FIELD (nT)

BOTTOM FIELD (nT)

GRAD.

\begin{tabular}{|c|c|c|c|c|c|c|c|c|c|c|c|c|}
\hline & & & & & & rr.) & 1 & (Diff.) & corr.) & Ir.) & (Diff.) & /nT/m \\
\hline 18 & & & & 1 & 2 & & 52026.8 & & 51973.0 & & -11.5 & $80^{\circ}$ \\
\hline 18 & 19 & 418 & 2568 & 51 & 2 & 102.6 & 2018.6 & -16.0 & 51963.4 & 51974.9 & -11.5 & -64.3 \\
\hline 18 & 195 & 132434 & 2570 & 51 & 9 & 69.4 & 85.4 & 0.0 & & 22.9 & 1.5 & -95.1 \\
\hline 18 & 195 & 132448 & 2572 & 1 & 18 & 51898.2 & 914.2 & -16.0 & & 847.1 & 1.5 & 02.6 \\
\hline 18 & 195 & 132503 & 2574 & 1 & 17 & 325.4 & 841.5 & -16.1 & 77.8 & 789.3 & 1.5 & 78.0 \\
\hline 18 & 195 & 132519 & 2576 & 1 & 16 & 50.6 & 1776.7 & -16.1 & & 1730.0 & -11.6 & -69.2 \\
\hline 18 & 195 & 132535 & 2578 & 1 & $\varepsilon$ & & 51722.1 & -16.1 & & 97.0 & -11.6 & -33.8 \\
\hline 18 & 195 & 132550 & 2580 & 1 & 14 & & & -16.1 & & 32.0 & -11.6 & -79.7 \\
\hline 18 & 35 & 132607 & 2582 & 1 & & & & -16.2 & 2.6 & & -11.6 & -34.4 \\
\hline 18 & 95 & 132624 & 884 & 1 & 2 & 51646.6 & 51662.8 & -16.2 & & & -11.6 & -20.0 \\
\hline 18 & 195 & 132643 & 86 & 1 & & 4.2 & & -16.2 & & & -11.7 & -11.8 \\
\hline 18 & 195 & 132703 & 2588 & 1 & 0 & 4.8 & & -16.3 & & & -11.7 & 6.6 \\
\hline 18 & 195 & 132728 & 2590 & 1 & & & & -16.3 & & & -11.7 & 5.2 \\
\hline 18 & 195 & 132744 & 992 & 1 & 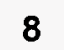 & & & -16.3 & & & -11.7 & -3.3 \\
\hline 18 & 195 & 132759 & & 1 & 7 & & & -16.4 & & & -11.7 & -1.3 \\
\hline 18 & 19 & 132814 & 2596 & 1 & 6 & 51 & 8.2 & -16.4 & & & -11.8 & -3.6 \\
\hline 18 & 195 & 132 & 98 & 1 & 5 & & & -16.4 & & .8 & -11.8 & -6.9 \\
\hline 18 & ( & 132 & 00 & 1 & 4 & & & -16.5 & & & 11.8 & 9.5 \\
\hline 18 & 195 & 132909 & 602 & 51 & 3 & & & & & & 11.8 & 9.3 \\
\hline 18 & 195 & 132925 & 04 & 1 & 2 & & & & & & 11.8 & -40.0 \\
\hline 18 & 195 & 132942 & 06 & 1 & 1 & & & -1 & & & 1.9 & -96.1 \\
\hline 18 & 195 & 133002 & 2608 & 51 & 0 & $?$ & & -16.6 & & & 11.9 & -310.8 \\
\hline 19 & 195 & 133421 & 2612 & 34 & 0 & & & & & & 11.8 & -91.2 \\
\hline 19 & & 133610 & & 24 & 1 & & & & & & 11.8 & -60.0 \\
\hline 10 & 19 & 133627 & 2616 & 54 & 2 & 51 & & & & & -11.8 & -48.9 \\
\hline 18 & 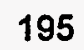 & 13 & 18 & & 3 & & & -1 & & & -11.8 & -37.0 \\
\hline 19 & 195 & 133 & 20 & 5 & 4 & & & -1 & & & -11.8 & -32.1 \\
\hline 19 & 195 & 133710 & 622 & 5 & 5 & & & & & & -11.8 & -23.0 \\
\hline 19 & 195 & 133723 & 2624 & 4 & 6 & 51 & & -162 & & & -11.7 & -23.0 \\
\hline 19 & 195 & & 2626 & 54 & 7 & & & -1 & & & -11.7 & -21.6 \\
\hline 19 & 195 & 133752 & 2628 & 54 & 8 & & & & & & 11.7 & 0.3 \\
\hline 19 & 195 & 133807 & 2630 & 54 & 9 & & & -16.1 & & & -11.7 & -20.0 \\
\hline 19 & 195 & 133821 & 2632 & 54 & 10 & & & -16.1 & & & -11.7 & -19.0 \\
\hline 10 & 1 & & 2634 & 54 & 11 & 5.6 & & -1 & & & -11.7 & -19.7 \\
\hline 19 & 195 & $13:$ & 2636 & 5 & $?$ & & & -1 & & & -11.7 & -22.6 \\
\hline 19 & & 13 & 8 & 54 & 3 & & & & & & -11.7 & -45.9 \\
\hline 19 & 195 & 13 & 10 & 54 & 4 & & & & & & -11.7 & -54.1 \\
\hline 19 & 195 & & 2642 & 54 & 15 & & & & & & -11.6 & 7.9 \\
\hline 19 & 195 & 133949 & 2644 & $\mathrm{~J}_{4}$ & 16 & & & & & & 1.6 & -28.2 \\
\hline 19 & & 134007 & 46 & 54 & 17 & & & -15.9 & & & -11.6 & -0.3 \\
\hline 19 & 19 & 134 & & 54 & 18 & & & -15.9 & 8.8 & & -11.6 & 105.2 \\
\hline 19 & 1 & & & 54 & 10 & & & -15.9 & & & -11.6 & -3.6 \\
\hline 18 & 19 & 134 & & 54 & & & & & & & -11.6 & -33.4 \\
\hline 19 & 195 & 134113 & 2654 & 54 & & 51 & & -1 & & & -11.6 & -6.2 \\
\hline 19 & 195 & 134128 & 2656 & 5 & 2 & & & & & & -11.6 & -24.9 \\
\hline 19 & 195 & 134143 & 2658 & $\mathrm{Ju}_{4}$ & 23 & & & & & & & -9.8 \\
\hline 19 & 195 & 134159 & 2660 & 54 & 24 & & & -15.7 & & 72.5 & -11.5 & -5.6 \\
\hline 19 & $19 !$ & 134213 & 2662 & 54 & 25 & & 51 & -15.7 & 44.8 & 756.3 & -11.5 & -8.2 \\
\hline 18 & 100 & 134229 & 2664 & 54 & 26 & 51728.2 & 51743.9 & -15.7 & 51736.4 & 51747.9 & -11.5 & 13.4 \\
\hline
\end{tabular}


TOP FIELD (nT)

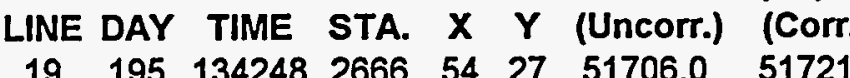

$\begin{array}{lllllll}19 & 195 & 134303 & 2668 & 54 & 28 & 51681.2\end{array}$

$\begin{array}{lllllll}19 & 195 & 134319 & 2670 & 54 & 29 & 51657.6\end{array}$

$\begin{array}{lllllll}19 & 195 & 134334 & 2672 & 54 & 30 & 51626.8\end{array}$

$\begin{array}{lllllll}19 & 195 & 134348 & 2674 & 54 & 31 & 51596.0\end{array}$

$\begin{array}{lllllll}19 & 195 & 134403 & 2676 & 54 & 32 & 51556.6\end{array}$

$\begin{array}{lllllll}19 & 195 & 134417 & 2678 & 54 & 33 & 51503.0\end{array}$

$\begin{array}{llllll}19 & 195 & 134434 & 2680 & 54 & 34\end{array}$

$\begin{array}{llll}19 & 195 & 134449 & 2682\end{array}$

$19 \quad 195 \quad 1345042684$

$\begin{array}{llll}19 & 195 & 134521 & 2686\end{array}$

$\begin{array}{llll}19 & 195 & 134536 & 2688\end{array}$

$\begin{array}{llll}19 & 195 & 134551 & 2690\end{array}$

$19 \quad 195 \quad 1346192692$

$\begin{array}{llll}19 & 195 & 134639 & 2694\end{array}$

$\begin{array}{llll}19 & 195 & 134707 & 2696\end{array}$

$\begin{array}{llll}19 & 195 & 134724 & 2698\end{array}$

$\begin{array}{llll}19 & 195 & 134740 & 2700\end{array}$

$\begin{array}{llll}19 & 195 & 134758 & 2702\end{array}$

$\begin{array}{llll}19 & 195 & 134813 & 2704\end{array}$

$\begin{array}{llll}19 & 195 & 134828 & 2706\end{array}$

$\begin{array}{llll}19 & 195 & 134843 \quad 2708\end{array}$

$\begin{array}{llll}19 & 195 & 134902 & 2710\end{array}$

$\begin{array}{llll}19 & 195 & 134918 & 2712\end{array}$

$\begin{array}{llll}19 & 195 & 134934 & 2714\end{array}$

$19 \quad 195 \quad 134948 \quad 2716$

$\begin{array}{llll}19 & 195 & 135003 & 2718\end{array}$

$\begin{array}{llll}19 & 195 & 135019 & 2720\end{array}$

$19 \quad 195 \quad 1350352722$

$\begin{array}{llll}19 & 195 & 135056 & 2724\end{array}$

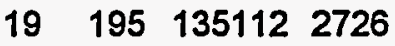

$\begin{array}{llll}19 & 195 & 135129 & 2728\end{array}$

$\begin{array}{llll}19 & 195 & 135147 & 2730\end{array}$

$\begin{array}{llll}19 & 195 & 135205 & 2732\end{array}$

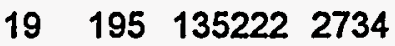

$\begin{array}{llll}19 & 195 & 135237 & 2736\end{array}$

$\begin{array}{llll}19 & 195 & 135252 & 2738\end{array}$

$\begin{array}{llll}19 & 195 & 135306 & 2740\end{array}$

$\begin{array}{llll}19 & 195 & 135322 & 2742\end{array}$

$\begin{array}{llll}19 & 195 & 135336 & 2744\end{array}$

$\begin{array}{llll}19 & 195 & 135352 & 2746\end{array}$

$\begin{array}{llll}19 & 195 & 135429 & 2748\end{array}$

$\begin{array}{llll}19 & 195 & 135449 & 2750\end{array}$

$19 \quad 195 \quad 135504 \quad 2752$

$\begin{array}{llll}19 & 195 & 135529 & 2754\end{array}$

$\begin{array}{llll}19 & 195 & 135547 & 2756\end{array}$

$\begin{array}{llll}19 & 195 & 135608 & 2758\end{array}$

$19195 \quad 135624 \quad 2760$

19

\section{$\begin{array}{lll}54 & 35 & 51299.0\end{array}$}

$\begin{array}{lll}54 & 36 & ? 51161\end{array}$

$\begin{array}{lll}54 & 37 & ? 51109.4\end{array}$

5438

5439

$54 \quad 40$

$54 \quad 41$

$\begin{array}{lll}54 & 42\end{array}$

5443

$\begin{array}{lll}54 & 44\end{array}$

$54 \quad 45$

$\begin{array}{lll}54 & 46 & ? 52167.8\end{array}$

$\begin{array}{llll}54 & 47 & ? 52168.8\end{array}$

$\begin{array}{lll}54 & 48 & 51963.8\end{array}$

$\begin{array}{llll}54 & 49 & 51627.0\end{array}$

$\begin{array}{lll}54 & 50 & 51265.8\end{array}$

$\begin{array}{lll}54 & 51 & ? 50931.2\end{array}$

$\begin{array}{lll}54 & 52 & ? 50797.8\end{array}$

$\begin{array}{lll}54 & 53 & ? 50819\end{array}$

$\begin{array}{lll}54 & 54 & ? 51062.8\end{array}$

$\begin{array}{lll}54 & 55 & ? 51749.8\end{array}$

$\begin{array}{lll}54 & 56 & ? 52671\end{array}$

$\begin{array}{lll}54 & 57 & ? 53365.2\end{array}$

$\begin{array}{lll}54 & 58 & ? 53120.6\end{array}$

$\begin{array}{lll}54 & 59 & ? 52771.4\end{array}$

$\begin{array}{lll}54 & 60 & 52273.2\end{array}$

$\begin{array}{llll}54 & 61 & 52018.4\end{array}$

$\begin{array}{lll}54 & 62 & 51872.6\end{array}$

5463

5464

5465

5466

5467

5468

$\begin{array}{ll}54 & 69\end{array}$

5470

5471

5472

?50493.4

$\begin{array}{lll}54 & 73 & ? 51258\end{array}$

$\begin{array}{lll}54 & 74 & ? 51708.6\end{array}$

$\begin{array}{lll}54 & 75 & ? 52186.8\end{array}$
$6-15.6$

$51696.8-15.6$

$51673.2-15.6$

$51642.3-15.5$

$51611.5-15.5$

$51572.1-15.5$

$51518.5-15.5$

$51442.2-15.4$

$51314.4 \quad-15.4$

$51176.4 \quad-15.4$

$51124.8-15.4$

$51197.1-15.3$

$51372.5-15.3$

$51586.7-15.3$

$51606.6-15.2$

$51484.8-15.2$

$51450.3-15.1$

$51556.7-15.1$

$51864.1-15.1$

$52182.8-15.0$

$52183.8-15.0$

$51978.8-15.0$

$\begin{array}{lll}51642 & -15.0\end{array}$

$51280.7-14.9$

$50946.1-14.9$

$50812.7-14.9$

$50833.8-14.8$

$\begin{array}{lll}51077.6 & -14.8\end{array}$

$\begin{array}{lll}51764.6 & -14.8\end{array}$

$\begin{array}{lll}52685.8 & -14.8\end{array}$

$\begin{array}{lll}53379.9 & -14.7\end{array}$

$53135.3-14.7$

$\begin{array}{lll}52786.1 & -14.7\end{array}$

$52287.8-14.6$

$52033-14.6$

$51887.2-14.6$

$51814.3-14.5$

$51774.3-14.5$

$51743.9-14.5$

$51723.5-14.5$

$51703.6-14.4$

$\begin{array}{lll}51677.4 & -14.4\end{array}$

$51632.3-14.3$

$51552.9-14.3$

$51338.3-14.3$

$50507.6-14.2$

$51272.2-14.2$

$\begin{array}{lll}51722.8 & -14.2\end{array}$

$52200.9-14.1$
BOTTOM FIELD (nT)

GRAD.

(Uncorr.) (Corr.) (Diff.) (nT/m)

$\begin{array}{llll}51704.6 & 51716.1 & -11.5 & -2.3\end{array}$

$51677.2 \quad 51688.7 \quad-11.5 \quad-6.6$

$\begin{array}{llll}51682.6 & 51694.1 & -11.5 & 41.0\end{array}$

$\begin{array}{lllll}51610.0 & 51621.5 & -11.5 & -27.5\end{array}$

$\begin{array}{llll}51596.4 & 51607.9 & -11.5 & 0.7\end{array}$

$51569.8 \quad 51581.2 \quad-11.4 \quad 21.6$

$\begin{array}{llll}51504.6 & 51516.0 & -11.4 & 2.6\end{array}$

$51448.0 \quad 51459.4 \quad-11.4 \quad 34.8$

$51225.2 \quad 51236.6 \quad-11.4 \quad-121.0$

$\begin{array}{lllll}? 50711.4 & 50722.8 & -11.4 & -737.0\end{array}$

$\begin{array}{lllll}\text { ?50763.4 } & 50774.8 & -11.4 & -567.2\end{array}$

$\begin{array}{lllll}250918 & 50929.4 & -11.4 & -432.5\end{array}$

$\begin{array}{llll}51322.8 & 51334.2 & -11.4 & -56.4\end{array}$

$\begin{array}{lllll}? 51824 & 51835.3 & -11.3 & 414.1\end{array}$

$\begin{array}{lllll}251845.8 & 51857.1 & -11.3 & 417.0\end{array}$

$\begin{array}{lllll}51460.6 & 51471.9 & -11.3 & -14.8\end{array}$

$51279.2 \quad 51290.5 \quad-11.3 \quad-255.7$

$\begin{array}{lllll}? 51254.2 & 51265.5 & -11.3 & -471.2\end{array}$

$\begin{array}{lllll}? 51590.8 & 51602.1 & -11.3 & -423.3\end{array}$

$\begin{array}{llll}? 52475.6 & 52486.8 & -11.2 & 504.6\end{array}$

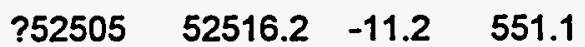

$\begin{array}{llll}? 52170.4 & 52181.6 & -11.2 & 338.7\end{array}$

$\begin{array}{llll}51682.8 & 51694.0 & -11.2 & 91.5\end{array}$

$\begin{array}{lllll}251118.6 & 51129.8 & -11.2 & -241.3\end{array}$

$\begin{array}{lllll}? 50525.6 & 50536.8 & -11.2 & -664.9\end{array}$

$\begin{array}{lllll}? 50184.6 & 50195.8 & -11.2 & -1005.3\end{array}$

$\begin{array}{lllll}? 50148.2 & 50159.4 & -11.2 & -1099.7\end{array}$

$\begin{array}{lllll}? 50370.6 & 50381.8 & -11.2 & -1134.8\end{array}$

$\begin{array}{lllll}251526.4 & 51537.5 & -11.1 & -366.2\end{array}$

$\begin{array}{llll}? 53085.8 & 53096.9 & -11.1 & 680.0\end{array}$

$\begin{array}{lllll}? 54493.8 & 54504.9 & -11.1 & 1850.2\end{array}$

$\begin{array}{lllll}253872.4 & 53883.5 & -11.1 & 1232.5\end{array}$

$\begin{array}{lllll}252828.6 & 52839.7 & -11.1 & 93.8\end{array}$

$\begin{array}{lllll}252153.4 & 52164.5 & -11.1 & -196.4\end{array}$

51906.0

$51917.1-11.1$

$-184.3$

51789.0

51745.4

51714.0

51695.2

51682.0

51666.8

51644.4

51606.0

51541.6

51376.2

$? 50871.4$

$? 50803.6$

?51053.8

$51800.0-11.0$

$-137.1$

$51756.4 \quad-11.0 \quad-89.2$

$51725.0-11.0 \quad-75.1$

$51706.2-11.0 \quad-56.1$

$51693.0 \quad-11.0 \quad-44.3$

$51677.8-11.0 \quad-36.7$

$\begin{array}{lll}51655.4 & -11.0 & -30.5\end{array}$

$51616.9 \quad-10.9 \quad-19.7$

$51552.5 \quad-10.9 \quad 4.9$

$51387.1-10.9 \quad 85.6$

$50882.3 \quad-10.9 \quad 619.7$

$\begin{array}{lll}50814.5 & -10.9 & -744.9\end{array}$

$51064.7 \quad-10.9 \quad-1073.4$

$\begin{array}{lllll}? 58613.6 & 58624.5 & -10.9 & 10535.7\end{array}$ 
TOP FIELD (nT)

\begin{tabular}{|c|c|c|c|c|c|c|c|c|c|c|c|c|}
\hline $\mathbf{v}$ & & 5711 & 2764 & 54 & & ?51038.4 & $\begin{array}{l}\text { (Corr.) } \\
51052.5\end{array}$ & $\begin{array}{l}\text { (Diff.) } \\
-14.1\end{array}$ & $\begin{array}{l}\text { (Uncorr.) } \\
\text { ?52105.8 }\end{array}$ & $\begin{array}{l}\text { (Corr.) } \\
52116.6\end{array}$ & $\begin{array}{l}\text { (Diff.) } \\
-10.8\end{array}$ & $\begin{array}{l}\text { (nT/m) } \\
1749.8\end{array}$ \\
\hline 19 & 195 & 135743 & 2766 & 54 & 77 & 51699.6 & 51713.6 & -14.0 & 51704.8 & 51715.6 & -10.8 & 8.5 \\
\hline 20 & 195 & 10119 & 2768 & 57 & 78 & 93.4 & 1707 & -13.6 & 686.8 & 1697.4 & -10.6 & -10.8 \\
\hline 20 & 195 & 140219 & 2770 & 57 & 77 & 51680.2 & 51693.7 & -13.5 & & 85.8 & -10.6 & -8.2 \\
\hline 20 & 195 & 140233 & 2772 & 57 & 76 & 1662.2 & 51675.7 & -13.5 & 51652.0 & 1662.6 & -10.6 & -16.7 \\
\hline 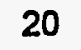 & 195 & 140247 & 2774 & 57 & 75 & 18.8 & 51632.3 & -13.5 & & 1593.8 & -10.6 & -58.4 \\
\hline 20 & 195 & 140303 & 2776 & 57 & 74 & 40.2 & 51553.6 & -13.4 & & 72.4 & -10.6 & -128.5 \\
\hline & 195 & 140319 & 2778 & 57 & 73 & ?51582.8 & 51596.2 & -13.4 & $? 51000.4$ & 1011.0 & -10.6 & -954.8 \\
\hline & 195 & 14 & 2780 & 57 & 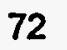 & 5.4 & 50788.8 & -13.4 & 32 & 1242.5 & -10.5 & 748.5 \\
\hline & 195 & 140403 & 2782 & 57 & 71 & 00.2 & 1413.5 & -13.3 & 4.2 & 84.7 & -10.5 & 121.3 \\
\hline & 195 & 140420 & 784 & 57 & 70 & 1573.8 & 1587.1 & -13.3 & & 99.7 & -10.5 & 25.2 \\
\hline & 195 & 40434 & 2786 & $5 /$ & 69 & 1.0 & 1654.3 & -13.3 & 2.2 & 52.7 & -10.5 & 2.0 \\
\hline 2 & 195 & 140447 & 2788 & 57 & 68 & 79.0 & 1692.3 & 3.3 & 2.4 & 32.9 & -10.5 & -10.8 \\
\hline 20 & 195 & 140504 & 2790 & 57 & 67 & 1704.0 & 51717.2 & -13.2 & 2.6 & 3.1 & -10.5 & -18.7 \\
\hline 20 & 195 & 140 & 2792 & 57 & 66 & 51726.0 & 51739.2 & -13.2 & 3.4 & 18.9 & -10.5 & -28.8 \\
\hline 20 & 195 & 140 & 2794 & 57 & 65 & 51748.8 & 51762 & -13.2 & 2.8 & 33.2 & -10.4 & -42.6 \\
\hline 20 & 195 & 140 & 2796 & 57 & 64 & 0.0 & 51789.1 & & & & -10.4 & -63.6 \\
\hline & 195 & 746 & 798 & 57 & 63 & 0.6 & 23.7 & -1 & .4 & 6.8 & -10.4 & -138.0 \\
\hline & 195 & 19 & 2800 & 57 & 62 & 32.2 & 1895.3 & .1 & 0.8 & 1.2 & -10.4 & -428.5 \\
\hline 2 & 195 & 140638 & 2802 & 57 & 61 & 51.8 & 2064.9 & .1 & & 9.0 & -10.4 & -169.2 \\
\hline$?$ & 195 & 00 & 2804 & 57 & 60 & 2287.8 & 2300.8 & -13.0 & & 7.2 & -10.4 & -132.8 \\
\hline$?$ & 195 & 140 & 2806 & 57 & 59 & 52663.2 & 52676.2 & -13.0 & & 4.8 & -10.4 & 67.5 \\
\hline$?$ & 195 & 140732 & 2808 & 57 & 58 & ?53069.8 & 53082.8 & .0 & & 3.6 & -10.4 & 759.7 \\
\hline$?$ & 195 & 140748 & 2810 & 57 & 57 & ?53132 & 4.9 & -1 & & 5.9 & -10.3 & 6202.6 \\
\hline & 195 & 140806 & 2812 & 57 & 56 & ?52769.6 & 52782.5 & -1 & & & -10.3 & 531.8 \\
\hline 20 & 195 & 140822 & 2814 & 57 & 55 & 52101.8 & 52114.7 & -12.9 & & & -10.3 & 168.2 \\
\hline 20 & 195 & 140 & 2816 & 57 & 54 & 0.0 & 51622.8 & -1 & & 6.7 & -10.3 & -415.7 \\
\hline 20 & 195 & 140 & 2818 & 57 & 53 & 9.0 & 1471.8 & & & 9.5 & -10.3 & 82.3 \\
\hline 20 & 195 & 14 & 2820 & 57 & 52 & 51342.2 & 51355 & & & 1.7 & -10.3 & -50.5 \\
\hline 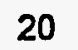 & 195 & 140 & 2822 & 57 & 51 & $? 51183.6$ & 51196.3 & .7 & $? 5$ & 20.5 & -10.3 & -612.1 \\
\hline & 195 & 14 & 2824 & 57 & 50 & 04.4 & 7.1 & .7 & $? 5$ & 3.6 & -10.2 & -559.0 \\
\hline & 195 & & 2826 & 57 & 49 & 51 & 51422.3 & .7 & & 1.0 & -10.2 & -145.6 \\
\hline$?$ & 195 & & 2828 & 57 & 48 & 51645.4 & 51658.1 & .7 & & & -10.2 & 85.2 \\
\hline 2 & 195 & & 2830 & 57 & 47 & 51807.6 & 51820.2 & & & & -10.2 & 233.8 \\
\hline 2 & 195 & 141055 & 2832 & 57 & 46 & 51877.2 & 51889.8 & -12.6 & & 2.6 & -10.2 & 320.0 \\
\hline 20 & 195 & 141111 & 2834 & 57 & 45 & 51803.2 & 51815.8 & -12.6 & & & -10.2 & 313.1 \\
\hline 20 & 19 & & 2836 & 57 & 44 & 1560.8 & 51573.3 & & & 36.6 & -10.2 & 42.0 \\
\hline 20 & & & 2838 & 57 & 43 & $? 51254.4$ & 51266.9 & & & 38.4 & -10.2 & -534.8 \\
\hline - & 10 & & 2840 & 57 & 42 & 37.6 & 0.1 & & & 29.7 & -10.1 & -603.3 \\
\hline 0 & 1 & 14 & 28 & 57 & 41 & 24.2 & 6.6 & .4 & & 18.3 & -10.1 & -518.0 \\
\hline 20 & 19 & 141231 & 2844 & 57 & 40 & $? 51053.2$ & 51065.6 & -12.4 & $? 5$ & 7.9 & -10.1 & -435.1 \\
\hline 20 & 195 & 141246 & 2846 & 57 & 39 & 51148.6 & 51161 & & & 8.7 & -10.1 & -163.9 \\
\hline 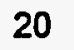 & 195 & 02 & 2848 & 57 & 38 & 51360.6 & 51373 & & & 77.5 & -10.1 & 339.0 \\
\hline 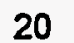 & 195 & & 2850 & 57 & 37 & 21.2 & 51433.5 & -12.3 & & 47.5 & -10.1 & 846.2 \\
\hline 20 & 195 & 141339 & 852 & 57 & 36 & 51259.6 & 51271.9 & -12.3 & 7.6 & 07.7 & -10.1 & -429.5 \\
\hline 20 & 195 & 141354 & 54 & 57 & 35 & 51245.0 & 51257.3 & -12.3 & & 31.1 & -10.1 & -203.3 \\
\hline 20 & & & 2856 & 57 & 34 & 51342.6 & 51354.8 & -12.2 & & 346.6 & -10.0 & -9.8 \\
\hline 20 & 19 & 141 & 2858 & 57 & 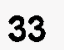 & 31.8 & & & & 65.0 & -10.0 & 38.0 \\
\hline & & & & & & & & -12.2 & 51519.8 & 51529.8 & -10.0 & 41.0 \\
\hline
\end{tabular}

BOTTOM FIELD (nT) GRAD. (nT/m) 8.5 $-10.8$ $-8.2$ $-16.7$ $-58.4$ $-128.5$ $-954.8$ 21.3 0 $-10.8$ $-18.7$ $-28.8$ $-42.6$ $-63.6$ $-138.0$ 28.5 32.8 67.5 202.6 31.8 82.3 59.0 45.6 33.8 20.0 42.0 534.8 $-518.0$ 35.1 (63.9 (3).0 29.5 03.3 41.0 
TOP FIELD (nT)

BOTTOM FIELD (nT)

GRAD.

\begin{tabular}{|c|c|c|c|c|c|c|c|c|c|c|c|c|}
\hline INE & AY & TIME & STA. & 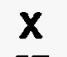 & 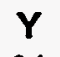 & ncorr.) & (Corr.) & (Diff.) & ) & - & (Diff.) & $\begin{array}{l}n T / m) \\
50.8\end{array}$ \\
\hline 20 & 195 & 141454 & 2862 & 57 & 31 & 51542.8 & 51555 & -12.2 & 51573.8 & 51583.8 & -10.0 & 50.8 \\
\hline 20 & 195 & 141508 & 2864 & 57 & 30 & 51573.6 & 51585.7 & -12.1 & 51601.8 & 51611.8 & -10.0 & 46.2 \\
\hline 20 & 195 & 141522 & 2866 & 57 & 29 & 51598.6 & 51610.7 & -12.1 & 51622.4 & 51632.4 & -10.0 & 39.0 \\
\hline 20 & 195 & 141536 & 2868 & 57 & 28 & 51617.8 & 51629.9 & -12.1 & 51647.0 & 51657.0 & -10.0 & 47.9 \\
\hline 20 & 195 & 141551 & 2870 & 57 & 27 & 51638.0 & 51650.1 & -12.1 & 51665.0 & 51675.0 & -10.0 & 44.3 \\
\hline 20 & 195 & 141614 & 2872 & 57 & 26 & 51658.8 & 51670.8 & -12.0 & 51686.2 & 51696.2 & -10.0 & 44.9 \\
\hline 20 & 195 & 141628 & 2874 & 57 & 25 & 51680.0 & 51692 & -12.0 & 51695.8 & 51705.7 & -9.9 & 25.9 \\
\hline 20 & 195 & 141642 & 2876 & 57 & 24 & 51697.8 & 51709.8 & -12.0 & 51725.6 & 51735.5 & -9.9 & 45.6 \\
\hline 20 & 195 & 141657 & 2878 & 57 & 23 & 51717.2 & 51729.1 & -11.9 & 51733.6 & 51743.5 & -9.9 & 26.9 \\
\hline 20 & 195 & 141712 & 2880 & 57 & 22 & 51729.4 & 51741.3 & -11.9 & 51753.0 & 51762.9 & -9.9 & 38.7 \\
\hline 20 & 195 & 141727 & 2882 & 57 & 21 & 51736.8 & 51748.7 & -11.9 & & 51766.3 & -9.9 & 32.1 \\
\hline 20 & 195 & 141742 & 2884 & 57 & 20 & 51740.0 & 51751.9 & -11.9 & 51 & 51762.3 & -9.9 & 20.3 \\
\hline 20 & 195 & 141756 & 886 & 57 & 19 & 51739.8 & 51751.6 & -11.8 & 9.8 & 59.7 & -9.9 & 16.4 \\
\hline 20 & 195 & 141810 & 2888 & 57 & 18 & 51 & 18.2 & -11.8 & & 5.7 & -9.9 & 15.4 \\
\hline 20 & 195 & 141824 & 2890 & 57 & 17 & 517 & 51742.6 & -11.8 & 2.0 & 51751.9 & -9.9 & 18.4 \\
\hline 20 & 195 & 141838 & 2892 & 57 & 16 & 517 & 51732.6 & -11.8 & 24.6 & 34.4 & -9.8 & 6.2 \\
\hline 20 & 195 & 141852 & 2894 & 57 & 15 & 517 & 51723.1 & -11.7 & & 51720.2 & -9.8 & -1.6 \\
\hline 20 & 195 & 141905 & 2896 & 57 & 14 & 517 & 51716.1 & -11.7 & & 51717.0 & -9.8 & 4.6 \\
\hline 20 & 195 & 141 & 2898 & 57 & 13 & 516 & 51710.9 & -11.7 & 2.2 & 2.0 & -9.8 & 4.9 \\
\hline 20 & 195 & 141 & 900 & 57 & 12 & 51 & 6.5 & -11.7 & 6 & 6.4 & -9.8 & 3.0 \\
\hline 20 & 195 & 141 & 902 & 57 & 11 & .0 & 1.6 & -11.6 & & 1.4 & -9.8 & 2.6 \\
\hline 20 & 195 & 142002 & 904 & 57 & 10 & .4 & 97 & -11.6 & & 6.6 & -9.8 & 2.3 \\
\hline 20 & 195 & 142017 & 906 & 57 & 9 & 51 & 3.2 & -11.6 & & 0.8 & -9.8 & -1.0 \\
\hline 20 & 195 & 142032 & 2908 & 57 & 8 & 51 & 38.2 & -11.6 & & 0.6 & -9.8 & 0.3 \\
\hline 20 & 195 & 142046 & 2910 & 57 & 7 & 51 & 33.1 & -11.5 & .6 & 1.3 & -9.7 & 0.0 \\
\hline 20 & 195 & 142101 & 912 & 57 & 6 & $51 \epsilon$ & 51676.9 & -11.5 & & 3.3 & -9.7 & -3.0 \\
\hline 20 & 195 & 142116 & 2914 & 57 & 5 & 516 & 51668.7 & -11.5 & & 2.9 & -9.7 & -6.6 \\
\hline 20 & 195 & 142130 & 916 & 57 & 4 & 516 & 51659.3 & -11.5 & & 9.3 & -9.7 & -13.4 \\
\hline 20 & 195 & 142 & 18 & 57 & 3 & 516 & 51644.6 & -11.4 & & 1.1 & -9.7 & -19.3 \\
\hline 20 & 195 & 142159 & 920 & 57 & 2 & 51 & 22 & -11.4 & 5.8 & 6.5 & -9.7 & -39.0 \\
\hline 20 & 195 & 142212 & 922 & 57 & 1 & 51 & 78 & -11.4 & 2.8 & 2.5 & -9.7 & -88.2 \\
\hline 20 & $19 !$ & 142226 & 924 & 57 & 0 & 1.8 & 13.2 & -11.4 & & 8.5 & -9.7 & -332.8 \\
\hline 21 & 195 & 144852 & 928 & 60 & 0 & 51 & 11.3 & -4.1 & .2 & 8.1 & -2.9 & -85.2 \\
\hline 21 & 195 & 144905 & 330 & 60 & 1 & 51 & 06.2 & -4.0 & & 5.6 & -2.8 & -64.6 \\
\hline 21 & 195 & 144917 & 932 & 60 & 2 & & 51634.5 & -3.9 & & 7.6 & -2.8 & -42.3 \\
\hline 21 & 195 & 144929 & 934 & 60 & 3 & 51650.2 & 51654.1 & -3.9 & 31.0 & 51633.7 & -2.7 & -31.5 \\
\hline 21 & 195 & 144952 & 936 & 60 & 4 & 51665.0 & 51668.8 & -3.8 & 18.8 & 51651.4 & -2.6 & -26.6 \\
\hline 21 & 19 & 145005 & 2938 & 60 & 5 & & 51677.5 & -3.7 & 2.0 & 34.6 & -2.6 & -19.3 \\
\hline 21 & 19 & 145017 & 340 & 60 & 6 & & 51 & -3.6 & & 5.1 & -2.5 & -15.4 \\
\hline 21 & 15 & 145030 & 942 & 60 & 7 & 51 & 1.2 & -3 & & 2.5 & -2.5 & -12.5 \\
\hline 21 & 19 & 145042 & 344 & 60 & 8 & & & -3.5 & & & -2.4 & -14.8 \\
\hline 21 & 195 & 145054 & 946 & 60 & 9 & 51 & 51702.7 & -3.5 & 1.6 & 4.0 & -2.4 & -12.5 \\
\hline 21 & 195 & 145106 & 2948 & 60 & 10 & 4.0 & 51707.4 & -3.4 & 9.4 & 51701.7 & -2.3 & -7.5 \\
\hline 21 & 195 & 145118 & 950 & 60 & 11 & 51709.6 & 51712.9 & -3.3 & 51705.4 & 51707.7 & -2.3 & -6.9 \\
\hline 21 & 195 & 145130 & 952 & 60 & 12 & 51715.4 & 51718.7 & -3.3 & 12.0 & 51714.2 & -2.2 & -5.6 \\
\hline 21 & & 145143 & 54 & 60 & 13 & 51 & 51722.6 & -3.2 & 18.0 & 51720.1 & -2.1 & -2.3 \\
\hline 21 & 19 & 145154 & 56 & 60 & 14 & 51722.8 & 51726 & -3.2 & 51720.4 & 51722.5 & -2.1 & -3.9 \\
\hline 21 & 19 & 145206 & 958 & 60 & 15 & & 9.5 & -3 & & 1.6 & -2.0 & -11.1 \\
\hline 21 & 195 & 145219 & 960 & 60 & 16 & 51733.2 & 51736.2 & -3.0 & 51739.2 & 51741.2 & -2.0 & 9.8 \\
\hline
\end{tabular}


TOP FIELD (nT)

BOTTOM FIELD (nT)

GRAD.

LINE DAY TIME STA. $X$ Y (Uncorr.) (Corr.) (Diff.) (Uncorr.) (Corr.) (Diff.) (nT/m)

\begin{tabular}{|c|c|c|c|c|c|c|c|c|c|c|c|c|}
\hline 21 & 195 & 145231 & 2962 & 60 & 17 & 51739.6 & 51742.6 & -3.0 & 51745.4 & 51747.3 & -1.9 & 9.5 \\
\hline 21 & 195 & 145243 & 2964 & 60 & 18 & 51742.4 & 51745.3 & -2.9 & 51752.6 & 51754.5 & -1.9 & 16.7 \\
\hline 21 & 195 & 145256 & 2966 & 60 & 19 & 51744.8 & 51747.7 & -2.9 & 51764.4 & 51766.2 & -1.8 & 32.1 \\
\hline 21 & 195 & 145309 & 2968 & 60 & 20 & 51741.6 & 51744.4 & -2.8 & 51763.8 & 51765.6 & -1.8 & 36.4 \\
\hline 21 & 195 & 145321 & 2970 & 60 & 21 & 51732.0 & 51734.8 & -2.8 & 51765.4 & 51767.1 & -1.7 & 54.8 \\
\hline 21 & 195 & 145334 & 2972 & 60 & 22 & 51712.8 & 51715.5 & -2.7 & 51758.0 & 51759.6 & -1.6 & 74.1 \\
\hline 2 & 195 & 145347 & 2974 & 60 & 23 & 51683.8 & 51686.4 & -2.6 & 51749.2 & 1750.8 & -1.6 & 107.2 \\
\hline 2 & 195 & 145359 & 2976 & 60 & 24 & 51637.0 & 51639.6 & -2.6 & 51705.0 & 1706.5 & -1.5 & 11.5 \\
\hline 2 & 195 & 145411 & 2978 & 60 & 25 & 51592.6 & 51595.1 & -2.5 & 51666.8 & 1668.3 & -1.5 & 121.6 \\
\hline 1 & 195 & 145424 & 2980 & 60 & 26 & 51542.4 & 51544.9 & -2.5 & 51595.6 & 1597.0 & -1.4 & 87.2 \\
\hline 2 & 195 & 145436 & 2982 & 60 & 27 & 51502.8 & 51505.2 & -2.4 & 51550.6 & 51552.0 & -1.4 & 78.4 \\
\hline & 195 & 145448 & 2984 & 60 & 28 & 51487.2 & 51489.5 & -2.3 & 51524.2 & 51525.5 & -1.3 & 60.7 \\
\hline & 195 & 145459 & 2986 & 60 & 29 & 51480.6 & 51482.9 & -2.3 & 51509.8 & 51511.1 & -1.3 & 47.9 \\
\hline 2 & 195 & 145515 & 2988 & 60 & 30 & 51476.0 & 51478.2 & -2.2 & 51497.2 & 51498.4 & -1.2 & 34.8 \\
\hline 2 & 195 & 145527 & 2990 & 60 & 31 & 51467.6 & 51469.8 & -2.2 & 51484.4 & 51485.5 & -1.1 & 27.5 \\
\hline & 195 & 145539 & 2992 & 60 & 32 & 51439.6 & 51441.7 & -2.1 & 51445.6 & 51446.7 & -1.1 & 9.8 \\
\hline & 19 & 145551 & 994 & 60 & 33 & 51390.4 & 51392.4 & -2.0 & 51383.4 & 51384.4 & -1.0 & -11.5 \\
\hline & 15 & 145604 & 996 & 60 & 34 & 51325.0 & 51327 & -2.0 & 51289.8 & 51290.8 & -1.0 & -57.7 \\
\hline & 19 & 145616 & 998 & 60 & 35 & 51244.2 & 51246.1 & -1.9 & 28.6 & 29.5 & -0.9 & -189.5 \\
\hline 2 & 195 & 145630 & 3000 & 60 & 36 & 51204.8 & 51206.7 & -1.9 & ?51009.6 & 51010.5 & -0.9 & -320.0 \\
\hline 2 & 195 & 145643 & 3002 & 60 & 37 & 51216.2 & 51218 & -1.8 & ?51297 & 51297.8 & -0.8 & 132.5 \\
\hline & 195 & 145657 & 3004 & 60 & 38 & 51043.4 & 51045.1 & -1.7 & ?50786.2 & 50786.9 & -0.7 & -421.6 \\
\hline & 195 & 145715 & 3006 & 60 & 39 & $? 50868$ & 50869.6 & -1.6 & $? 50398$ & 50398.7 & -0.7 & -770.5 \\
\hline & 195 & 145729 & 3008 & 60 & 40 & $? 50926$ & 50927.6 & -1.6 & $? 50520.8$ & 50521.4 & -0.6 & -664.3 \\
\hline & 195 & 145741 & 3010 & 60 & 41 & 51152.6 & 51154.1 & -1.5 & 51009.0 & 51009.5 & -0.5 & -235.4 \\
\hline 2 & 195 & 145754 & 3012 & 60 & 42 & 51450.6 & 51452.1 & -1.5 & ?51514.2 & 51514.7 & -0.5 & 104.3 \\
\hline 2 & 195 & 145807 & 3014 & 60 & 43 & 51620.4 & 51621.8 & -1.4 & 51698.4 & 51698.8 & -0.4 & 127.9 \\
\hline 2 & 195 & 145819 & 3016 & 60 & 44 & 51675.4 & 51676.7 & -1.3 & $? 51644.2$ & 51644.6 & -0.4 & -51.2 \\
\hline 2 & 195 & 145832 & 3018 & 60 & 45 & 51693.0 & 51694.3 & -1.3 & $? 51526.4$ & 51526.7 & -0.3 & -273.1 \\
\hline 2 & 19 & 145845 & 3020 & 60 & 46 & 51710.0 & 51711.2 & -1.2 & 570 & 51570.3 & -0.3 & -229.5 \\
\hline 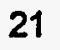 & 19 & 145858 & 3022 & 60 & 47 & 51719.4 & 51720.5 & -1.1 & 3.2 & 53.4 & -0.2 & -108.5 \\
\hline 2 & 195 & 145910 & 3024 & 60 & 48 & 51665.4 & 6.5 & -1.1 & 4.8 & 51644.9 & -0.1 & -33.8 \\
\hline 2 & 195 & 145923 & 3026 & 60 & 49 & 51521.4 & 22.4 & -1.0 & 71.6 & 71.7 & -0.1 & -81.6 \\
\hline 2 & 195 & 145935 & 3028 & 60 & 50 & 51323.8 & 51324.8 & -1.0 & $? 51107.8$ & 51107.8 & 0.0 & -354.1 \\
\hline 2 & 195 & 145950 & 3030 & 60 & 51 & ?51168.4 & 51169.3 & -0.9 & $? 50860.4$ & 50860.4 & 0.0 & -504.9 \\
\hline 2 & 195 & 150005 & 3032 & 60 & 52 & $? 51096$ & 51096.8 & -0.8 & $? 50896.6$ & 50896.5 & 0.1 & -326.9 \\
\hline 21 & 195 & 150025 & 3034 & 60 & 53 & $? 51100.2$ & 51100.9 & -0.7 & $? 50800.8$ & 50800.6 & 0.2 & -490.8 \\
\hline 21 & 195 & 150039 & 3036 & 60 & 54 & $? 51186$ & 51186.7 & -0.7 & $? 50749$ & 50748.8 & 0.2 & -716.4 \\
\hline 21 & 195 & 150054 & 3038 & 60 & 55 & $? 51559$ & 51559.6 & -0.6 & ?51171.8 & 51171.5 & 0.3 & -634.8 \\
\hline ) & 95 & 150107 & 3040 & 60 & 56 & $? 52190.8$ & 52191.3 & -0.5 & ?52279.4 & 52279.0 & 0.4 & 145.2 \\
\hline & 195 & 150121 & 3042 & 60 & 57 & ?52736.2 & 52736.7 & -0.5 & ?53359.8 & 53359.4 & 0.4 & 1022.3 \\
\hline & 195 & 150150 & 3044 & 60 & 58 & ?52890.8 & 52891.1 & -0.3 & ?53328.8 & 53328.2 & 0.6 & 718.0 \\
\hline & 195 & 150225 & 3046 & 60 & 59 & 52682.8 & 52683 & -0.2 & 52784.0 & 52783.3 & 0.7 & 165.9 \\
\hline 21 & 195 & 150237 & 3048 & 60 & 60 & 52360.4 & 52360.5 & -0.1 & 52290.0 & 52289.2 & 0.8 & -115.4 \\
\hline 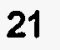 & 195 & 150249 & 3050 & 60 & 61 & 52136.6 & 52136.6 & 0.0 & 52043.0 & 52042.2 & 0.8 & -153.4 \\
\hline-1 & 195 & 150304 & 3052 & 60 & 62 & 51996.0 & 51996 & 0.0 & 51922.0 & 51921.1 & 0.9 & -121.3 \\
\hline 2 & 195 & 150315 & 3054 & 60 & 63 & 51901.4 & 51901.3 & 0.1 & 51843.2 & 51842.3 & 0.9 & -95.4 \\
\hline 21 & 19 & 150327 & 3056 & 60 & 64 & 51843.2 & 51843.1 & 0.1 & 51792.2 & 51791.2 & 1.0 & -83.6 \\
\hline & 195 & 150339 & 58 & 60 & 65 & 51798.8 & 51798.6 & 0.2 & 51760.6 & 51759.5 & 1.1 & -62.6 \\
\hline
\end{tabular}


.TOP FIELD (nT)

BOTTOM FIELD (nT)

GRAD.

LINE DAY TIME STA. $X \quad Y$ (Uncorr.) (Corr.) (Diff.) (Uncorr.) (Corr.) (Diff.) (nT/m)

$\begin{array}{llllllllllll}195 & 150355 & 3060 & 60 & 66 & 51769.6 & 51769.3 & 0.3 & 51739.4 & 51738.3 & 1.1 & -49.5\end{array}$

$\begin{array}{lllllllllllll}21 & 195 & 150406 & 3062 & 60 & 67 & 51743.8 & 51743.5 & 0.3 & 51719.2 & 51718.0 & 1.2 & -40.3\end{array}$

$\begin{array}{llllllllllllll}21 & 195 & 150418 & 3064 & 60 & 68 & 51713.6 & 51713.2 & 0.4 & 51697.0 & 51695.8 & 1.2 & -27.2\end{array}$

$\begin{array}{lllllllll}21 & 195 & 150432 & 3066 & 60 & 69 & 51674.0 & 51673.6 & 0.4\end{array}$

$\begin{array}{llllllllll}21 & 195 & 150444 & 3068 & 60 & 70 & 51600.6 & 51600.1 & 0.5\end{array}$

$\begin{array}{llllllllll}21 & 195 & 150501 & 3070 & 60 & 71 & 51402.2 & 51401.6 & 0.6\end{array}$

$\begin{array}{llllllllll}21 & 195 & 150514 & 3072 & 60 & 72 & ? 50510.2 & 50509.6 & 0.6\end{array}$

$\begin{array}{lllllllll}21 & 195 & 150527 & 3074 & 60 & 73 & 251754.2 & 51753.5 & 0.7\end{array}$

$\begin{array}{llllllllll}21 & 195 & 150540 & 3076 & 60 & 74 & 751435.6 & 51434.8 & 0.8\end{array}$

$\begin{array}{llllllllll}21 & 195 & 150553 & 3078 & 60 & 75 & 51597.2 & 51596.4 & 0.8\end{array}$

$\begin{array}{lllllllll}21 & 195 & 150606 & 3080 & 60 & 76 & 51664.8 & 51663.9 & 0.9\end{array}$

$\begin{array}{lllllllll}21 & 195 & 150617 & 3082 & 60 & 77 & 51696.2 & 51695.3 & 0.9\end{array}$

$\begin{array}{lllllllll}21 & 195 & 150630 & 3084 & 60 & 78 & 51711.4 & 51710.4 & 1.0\end{array}$

$\begin{array}{lllllllll}21 & 195 & 150642 & 3086 & 60 & 79 & 51720.4 & 51719.3 & 1.1\end{array}$

$\begin{array}{lllllllll}22 & 195 & 151040 & 3088 & 63 & 68 & 51721.2 & 51719 & 2.2\end{array}$

$\begin{array}{lllllll}22 & 195 & 151055 & 3090 & 63 & 67 & 51745.0\end{array}$

$\begin{array}{lllllll}22 & 195 & 151107 & 3092 & 63 & 66 & 51767.4\end{array}$

$\begin{array}{lllllll}22 & 195 & 151119 & 3094 & 63 & 65 & 51800.4\end{array}$

$\begin{array}{lllllll}22 & 195 & 151132 & 3096 & 63 & 64 & 51839.6\end{array}$

$\begin{array}{lllllll}22 & 195 & 151143 & 3098 & 63 & 63 & 51897.6\end{array}$

$\begin{array}{lllllll}22 & 195 & 151157 & 3100 & 63 & 62 & 51994.8\end{array}$

$\begin{array}{lllllll}22 & 195 & 151209 & 3102 & 63 & 61 & 52136.6\end{array}$

$\begin{array}{lllllll}22 & 195 & 151222 & 3104 & 63 & 60 & 52404.2\end{array}$

$\begin{array}{lllllll}22 & 195 & 151234 & 3106 & 63 & 59 & ? 52815\end{array}$

$\begin{array}{lllllll}22 & 195 & 151248 & 3108 & 63 & 58 & ? 53104\end{array}$

$\begin{array}{lllllll}22 & 195 & 151300 & 3110 & 63 & 57 & \text { ?52941.2 }\end{array}$

$\begin{array}{lllllll}22 & 195 & 151313 & 3112 & 63 & 56 & 252338.8\end{array}$

$\begin{array}{lllllll}22 & 195 & 151325 & 3114 & 63 & 55 & ? 51482.8\end{array}$

$\begin{array}{lllllll}22 & 195 & 151338 & 3116 & 63 & 54 & ? 50798.8\end{array}$

$\begin{array}{lllllll}22 & 195 & 151351 & 3118 & 63 & 53 & ? 50573.2\end{array}$

$\begin{array}{lllllll}22 & 195 & 151407 & 3120 & 63 & 52 & ? 50803.6\end{array}$

$\begin{array}{lllllll}22 & 195 & 151420 & 3122 & 63 & 51 & ? 51128.6\end{array}$

$\begin{array}{lllllll}22 & 195 & 151433 & 3124 & 63 & 50 & 51419.8\end{array}$

$\begin{array}{llllllll}22 & 195 & 151445 & 3126 & 63 & 49 & 51739.6\end{array}$

$\begin{array}{lllllll}22 & 195 & 151505 & 3128 & 63 & 48 & 52051.6\end{array}$

$\begin{array}{lllllll}22 & 195 & 151518 & 3130 & 63 & 47 & ? 52256.6\end{array}$

$\begin{array}{lllllll}22 & 195 & 151532 & 3132 & 63 & 46 & ? 52363.8\end{array}$

$\begin{array}{lllllll}22 & 195 & 151548 & 3134 & 63 & 45 & ? 52420.6\end{array}$

$\begin{array}{lllllll}22 & 195 & 151603 & 3136 & 63 & 44 & ? 52467\end{array}$

$\begin{array}{lllllll}22 & 195 & 151617 & 3138 & 63 & 43 & ? 52509.6\end{array}$

$\begin{array}{lllllll}22 & 195 & 151632 & 3140 & 63 & 42 & ? 52287.8\end{array}$

$51742.7 \quad 2.3$

51765.12 .3

$\begin{array}{ll}51798 \quad 2.4 \\ 51837.1 & 2.5\end{array}$

51837.12 .5

51895.12 .5

51992.22 .6

$52134 \quad 2.6$

$\begin{array}{ll}52401.5 & 2.7\end{array}$

$52812.3 \quad 2.7$
53101.2

51664.0

5169

$-16.4$

51603.8

51454.6

51602.5

1.3

5.2

$? 50767.2$

?49978.2

51453.2

1.4

85.9

$? 51220.8$

40765.7

1.5

421.3

51554.8

4976.7

1.5

$-2911.5$

51639.6

51553.2

1.6

$-352.1$

51675.2

51637.9

1.6

$-69.5$

51697.2

51673.4

1.7

$-41.3$

51708.6

51695.4

51704.4

51706.7

1.8

$-34.4$

51726.4

51701.5

1.8

$-23.3$

51744.8

51723.4

1.9

$-19.3$

51771.2

51741.7

2.9

$-27.5$

51802.6

51768.1

3.0

$-30.5$

51851.2

51799.4

3.1

$-37.1$

51933.2

51848.0

3.1

$-47.9$

52060.8

51929.9

3.2

$-60.7$

52386.2

52057.5

3.2

$-76.1$

$53101.2 \quad 2.8$

$52938.3 \quad 2.9$

52335.92 .9

$51479.8 \quad 3.0$

$50795.7 \quad 3.1$

50570.13 .1

$50800.4 \quad 3.2$

$51125.3 \quad 3.3$

$51416.5 \quad 3.3$

$51736.2 \quad 3.4$

52048.13 .5

52253.13 .5

$52360.2 \quad 3.6$

52416.93 .7

$52463.3 \quad 3.7$

253126.8

52382.8

3.3

$-101.0$

?53871.6

53123.4

3.3

$-124.3$

?53413.8

53868.1

3.4

$-29.5$

?52294.6

53410.2

3.4

511.1

?50787.8

52291.0

3.5

1258.4

?49901.4

50784.1

3.6

774.8

?49808.4

49897.7

3.6

$-72.5$

$? 50356$

49804.6

3.7

$-1139.3$

?50933.8

50352.2

3.7

$-1471.2$

?51282.4

50929.9

$3.8-1253.8$

$52505.8 \quad 3.8$

$52283.9 \quad 3.9$

$51773 \quad 4.0$

$\begin{array}{llll}22 & 195 & 151646 & 3142\end{array}$

$\begin{array}{llll}63 & 41 & 51777.0\end{array}$

51235.24 .0

51764.4

?52257.4

51278.4

$3.8-733.8$

?52574

51760.4

$3.9-319.3$

?52613.6

52253.3

4.0

$-225.2$

?52639.6

52569.8

4.0
4.1

40.7

?52793.4

52609.4

4.2

337.4

?52810.2

52635.3

4.2

520.3

?52914.8

52789.0

4.3

409.5

?51878

52805.8

4.4

359.0

?50792.8

52910.3

4.4

535.1

?50513.8

51873.4

4.5

492.8

$\begin{array}{lllll}22 & 195 & 151714 & 3146\end{array}$

$\begin{array}{lll}63 & 40 & ? 51239.2 \\ 63 & 39 & ? 51019.2\end{array}$

51015.14 .1

50788.2

4.6

1027.9

?50969.8

50509.1

4.6
4.7

165.6

$51164.9 \quad 4.1$

?51798

51793.2

4.7

$-731.8$

$\begin{array}{llll}22 & 195 & 151739 & 3150\end{array}$

$\begin{array}{lll}63 & 37 & 51414.2\end{array}$

$51410 \quad 4.2$

?51206.4

51201.5

4.8

$-828.5$

$\begin{array}{llll}22 & 195 & 151753 & 3152 \\ 22 & 195 & 151805 & 3154\end{array}$

51364.94 .3

$? 51048.4$

51043.5

4.9

$-326.6$

22

$195 \quad 1518203156$

$\begin{array}{lll}63 & 35 & 51272.4 \\ 63 & 34 & 51300.0\end{array}$

$51295.6 \quad 4.4$

51217.2

51212.2

4.9

$-266.9$

$-367.2$

$-135.7$ 
TOP FIELD (nT)

BOTTOM FIELD (nT)

GRAD.

\begin{tabular}{|c|c|c|c|c|c|c|c|c|c|c|c|c|}
\hline 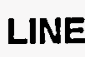 & AY & TIME & TA. & 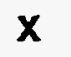 & $y$ & r.) & 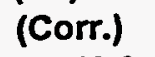 & (Diff.) & 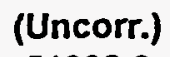 & orr.) & (Diff.) & (nT/m) \\
\hline 22 & 195 & 151833 & 3158 & 63 & 33 & 51353.8 & 51349.3 & 4.5 & 51336.2 & 51331.2 & 5.0 & -28.9 \\
\hline 22 & 195 & 151847 & 3160 & 63 & 32 & 51378.6 & 51374.1 & 4.5 & 51378.8 & 51373.7 & 5.1 & 0.3 \\
\hline 22 & 195 & 151900 & 3162 & 63 & 31 & 51357.6 & 51353 & 4.6 & 51358.2 & 51353.0 & 5.2 & 1.0 \\
\hline 22 & 195 & 151913 & 3164 & 63 & 30 & 51280.4 & 51275.7 & 4.7 & 51277.8 & 51272.6 & 5.2 & -4.3 \\
\hline 22 & 195 & 151925 & 3166 & 63 & 29 & 51138.2 & 51133.5 & 4.7 & 51127.8 & 51122.5 & 5.3 & -17.0 \\
\hline 22 & 195 & 151938 & 3168 & 63 & 28 & 50904.4 & 50899.6 & 4.8 & ?50885.6 & 50880.3 & 5.3 & -30.8 \\
\hline 22 & 195 & 151951 & 3170 & 63 & 27 & $? 50589.4$ & 50584.6 & 4.8 & $? 50530.8$ & 50525.4 & 5.4 & -96.1 \\
\hline 22 & 195 & 152005 & 3172 & 63 & 26 & $? 50402.6$ & 50397.7 & 4.9 & $? 50339$ & 50333.6 & 5.4 & -104.3 \\
\hline 22 & 195 & 152018 & 3174 & 63 & 25 & $? 50502.6$ & 50497.6 & 5.0 & $? 50641.6$ & 50636.1 & 5.5 & 227.9 \\
\hline 22 & 195 & 152031 & 3176 & 63 & 24 & ?51019.2 & 51014.2 & 5.0 & ?51340.2 & 51334.6 & 5.6 & 526.2 \\
\hline 22 & 195 & 152046 & 3178 & 63 & 23 & $? 51409.2$ & 51404.1 & 5.1 & ?51702.2 & 51696.6 & 5.6 & 480.3 \\
\hline 22 & 195 & 152105 & 3180 & 63 & 22 & 51611.4 & 51606.2 & 5.2 & ?51818.4 & 51812.7 & 5.7 & 339.3 \\
\hline 22 & 195 & 152121 & 3182 & 63 & 21 & 51701.4 & 51696.1 & 5.3 & 51828.0 & 51822.2 & 5.8 & 207.5 \\
\hline 22 & 195 & 152133 & 3184 & 63 & 20 & 51731.6 & 51726.3 & 5.3 & 51806.0 & 51800.2 & 5.8 & 122.0 \\
\hline 22 & 195 & 152146 & 3186 & 63 & 19 & 51739.8 & 51734.4 & 5.4 & 51789.4 & 51783.5 & 5.9 & 81.3 \\
\hline 22 & 195 & 152214 & 3188 & 63 & 18 & 51741.8 & 51736.3 & 5.5 & 51773.0 & 51767.0 & 6.0 & 51.1 \\
\hline 22 & 195 & 152243 & 3190 & 63 & 17 & 51740.0 & 51734.3 & 5.7 & 51763.6 & 51757.4 & 6.2 & 38.7 \\
\hline 22 & 195 & 152256 & 3192 & 63 & 16 & 51738.0 & 51732.3 & 5.7 & 51756.0 & 51749.8 & 6.2 & 29.5 \\
\hline 22 & 195 & 152308 & 3194 & 63 & 15 & 51734.8 & 51729 & 5.8 & 51748.0 & 51741.7 & 6.3 & 21.6 \\
\hline 22 & 195 & 152321 & 3196 & 63 & 14 & 51731.8 & 51726 & 5.8 & 51740.4 & 51734.1 & 6.3 & 14.1 \\
\hline 22 & 195 & 152333 & 3198 & 63 & 13 & 51727.6 & 51721.7 & 5.9 & 51734.0 & 51727.6 & 6.4 & 10.5 \\
\hline 22 & 195 & 152345 & 3200 & 63 & 12 & 51723.0 & 51717.1 & 5.9 & 51727.6 & 51721.2 & 6.4 & 7.5 \\
\hline 22 & 195 & 152359 & 3202 & 63 & 11 & 51718.0 & 51712 & 6.0 & 51722.0 & 51715.5 & 6.5 & 6.6 \\
\hline 22 & 195 & 152411 & 3204 & 63 & 10 & 51713.8 & 51707.7 & 6.1 & 51716.8 & 51710.3 & 6.5 & 4.9 \\
\hline 22 & 195 & 152423 & 3206 & 63 & 9 & 51709.0 & 51702.9 & 6.1 & 51711.2 & 51704.6 & 6.6 & 3.6 \\
\hline 22 & 195 & 152435 & 3208 & 63 & 8 & 51703.6 & 51697.4 & 6.2 & 4.0 & 51697.3 & 6.7 & 0.7 \\
\hline 22 & 195 & 152447 & 3210 & 63 & 7 & 51696.8 & 51690.6 & 6.2 & 6.0 & 9.3 & 6.7 & -1.3 \\
\hline 22 & 195 & 152459 & 3212 & 63 & 6 & 51689.2 & 51682.9 & 6.3 & 51687.0 & 0.2 & 6.8 & -3.6 \\
\hline 22 & 195 & 152512 & 3214 & 63 & 5 & 516 & 51674.2 & 6.4 & 0.2 & 9.4 & 6.8 & -7.2 \\
\hline 22 & 195 & 152538 & 3216 & 63 & 4 & 51669.8 & 51663.3 & 6.5 & 0.2 & 3.3 & 6.9 & -15.7 \\
\hline 22 & 195 & 152550 & 3218 & 63 & 3 & 51653.8 & 51647.3 & 6.5 & 8.2 & 1.2 & 7.0 & -25.6 \\
\hline 22 & 195 & 152603 & 3220 & 63 & 2 & 51627.6 & 51621 & 6.6 & 9.2 & 51592.2 & 7.0 & -46.6 \\
\hline 22 & 195 & 152615 & 3222 & 63 & 1 & 51578.0 & 51571.3 & 6.7 & 8.6 & 1.5 & 7.1 & -97.4 \\
\hline 22 & 195 & 152627 & 3224 & 63 & 0 & ?51454.4 & 51447.7 & 6.7 & 51358.0 & 51350.8 & 7.2 & -158.0 \\
\hline 23 & 195 & 153059 & 3228 & 66 & 0 & 51556.2 & 51549 & 7.2 & 51410.6 & 51403.0 & 7.6 & -238.7 \\
\hline 23 & 195 & 153112 & 3230 & 66 & 1 & 51608.4 & 51600.7 & 7.7 & 51553.2 & 51545.2 & 8.0 & -90.5 \\
\hline 23 & 195 & 153125 & 3232 & 66 & 2 & 51637.0 & 51629.3 & 7.7 & 51610.0 & 51601.9 & 8.1 & -44.3 \\
\hline 23 & 195 & 153136 & 3234 & 66 & 3 & 51651.2 & 51643.5 & 7.7 & 51637.6 & 51629.5 & 8.1 & -22.3 \\
\hline 23 & 195 & 153148 & 3236 & 66 & 4 & 51655.2 & 51647.4 & 7.8 & 51643.8 & 51635.7 & 8.1 & -18.7 \\
\hline 23 & 195 & 153201 & 3238 & 66 & 5 & 51661.0 & 51653.2 & 7.8 & 51646.8 & 8.6 & 8.2 & -23.3 \\
\hline 23 & 195 & 153214 & 3240 & 66 & 6 & 51676.0 & 51668.2 & 7.8 & 51659.0 & 51650.8 & 8.2 & -27.9 \\
\hline 23 & 195 & 153227 & 3242 & 66 & 7 & 51692.4 & 51684.5 & 7.9 & 51679.4 & 51671.2 & 8.2 & -21.3 \\
\hline 23 & 195 & 153239 & 3244 & 66 & 8 & 51705.4 & 51697.5 & 7.9 & 51696.4 & 51688.2 & 8.2 & -14.8 \\
\hline 23 & 195 & 153252 & 3246 & 66 & 9 & 51713.4 & 51705.5 & 7.9 & 51706.8 & 51698.5 & 8.3 & -10.8 \\
\hline 23 & 195 & 153306 & 3248 & 66 & 10 & 51718.2 & 51710.2 & 8.0 & 51714.4 & 51706.1 & 8.3 & -6.2 \\
\hline 23 & 195 & 153319 & 3250 & 66 & 11 & 51720.2 & 51712.2 & 8.0 & 51718.2 & 51709.9 & 8.3 & -3.3 \\
\hline 23 & 195 & 153332 & 3252 & 66 & 12 & 51720.4 & 51712.4 & 8.0 & 51711.4 & 51703.0 & 8.4 & -14.8 \\
\hline 23 & 195 & 153345 & 3254 & 66 & 13 & 51724.2 & 51716.1 & 8.1 & 51723.2 & 51714.8 & 8.4 & -1.6 \\
\hline$-\infty$ & 195 & 153357 & 3256 & 66 & 14 & 51727.6 & 51719.5 & 8.1 & 51732.8 & 51724.4 & 8.4 & 8.5 \\
\hline
\end{tabular}


TOP FIELD (nT)

LINE DAY TIME STA. $X Y$ (Uncorr.) (Corr.)

$\begin{array}{lllllll}23 & 195 & 153409 & 3258 & 66 & 15 & 51726.8\end{array}$

$\begin{array}{lllllll}23 & 195 & 153422 & 3260 & 66 & 16 & 51727.6\end{array}$

$\begin{array}{lllllll}23 & 195 & 153434 & 3262 & 66 & 17 & 51726.8\end{array}$

$\begin{array}{lllllll}23 & 195 & 153446 & 3264 & 66 & 18 & 51720.4\end{array}$

$\begin{array}{lllllll}23 & 195 & 153458 & 3266 & 66 & 19 & 51705.0\end{array}$

$\begin{array}{lllllll}23 & 195 & 153511 & 3268 & 66 & 20 & 51674.8\end{array}$

$\begin{array}{lllllll}23 & 195 & 153523 & 3270 & 66 & 21 & 51597.6\end{array}$

$\begin{array}{lllllll}23 & 195 & 153535 & 3272 & 66 & 22 & 51401.2\end{array}$

$\begin{array}{lllllll}23 & 195 & 153547 & 3274 & 66 & 23 & ? 50940.6\end{array}$

$\begin{array}{lllllll}23 & 195 & 153600 & 3276 & 66 & 24 & ? 49871.4\end{array}$

$\begin{array}{lllllll}23 & 195 & 153618 & 3278 & 66 & 25 & ? 58601.2\end{array}$

23

23

23

23

23

23

23

23

23

23

23

23

23

23

23

23

23

23

23

23

23

23

23

23

23

23

23

23

23

23

23

23

23

23

23

23

23

23 $\begin{array}{llllll}195 & 153650 & 3280 & 66 & 26 & ? 48059.8\end{array}$

$\begin{array}{llllll}195 & 153712 & 3282 & 66 & 27 & ? 49340.2\end{array}$

$\begin{array}{llllll}195 & 153728 & 3284 & 66 & 28 & ? 50639.6\end{array}$

$\begin{array}{llllll}195 & 153742 & 3286 & 66 & 29 & ? 51265.8\end{array}$

$\begin{array}{llllll}195 & 153755 & 3288 & 66 & 30 & 51476.2\end{array}$

$\begin{array}{llllll}195 & 153807 & 3290 & 66 & 31 & 51609.4\end{array}$

$\begin{array}{llllll}195 & 153818 & 3292 & 66 & 32 & 51604.0\end{array}$

$\begin{array}{llllll}195 & 153832 & 3294 & 66 & 33 & 51524.6\end{array}$

$\begin{array}{llllll}195 & 153845 & 3296 & 66 & 34 & 51443.0\end{array}$

$\begin{array}{llllll}195 & 153857 & 3298 & 66 & 35 & 51415.8\end{array}$

$\begin{array}{llllll}195 & 153914 & 3300 & 66 & 36 & 51569.8\end{array}$

$\begin{array}{llllll}195 & 153928 & 3302 & 66 & 37 & ? 51588.2\end{array}$

$\begin{array}{llllll}195 & 153942 & 3304 & 66 & 38 & 51319.8\end{array}$

$\begin{array}{llllll}195 & 153956 & 3306 & 66 & 39 & ? 51132.6\end{array}$

$\begin{array}{llllll}195 & 154008 & 3308 & 66 & 40 & ? 51201.6\end{array}$

$\begin{array}{llllll}195 & 154021 & 3310 & 66 & 41 & 51495.4\end{array}$

$\begin{array}{llllll}195 & 154035 & 3312 & 66 & 42 & 51845.6\end{array}$

$\begin{array}{llllll}195 & 154049 & 3314 & 66 & 43 & 52056.6\end{array}$

$\begin{array}{llllll}195 & 154102 & 3316 & 66 & 44 & 52272.0\end{array}$

$\begin{array}{llllll}195 & 154115 & 3318 & 66 & 45 & 52471.6\end{array}$

$\begin{array}{llllll}195 & 154127 & 3320 & 66 & 46 & 52600.6\end{array}$

$\begin{array}{llllll}195 & 154139 & 3322 & 66 & 47 & 52563.6\end{array}$

$\begin{array}{llllll}195 & 154151 & 3324 & 66 & 48 & 52346.0\end{array}$

$\begin{array}{llllll}195 & 154204 & 3326 & 66 & 49 & 52025.8\end{array}$

$\begin{array}{llllll}195 & 154217 & 3328 & 66 & 50 & 51791.4\end{array}$

$\begin{array}{llllll}195 & 154231 & 3330 & 66 & 51 & 51648.2\end{array}$

$\begin{array}{llllll}195 & 154244 & 3332 & 66 & 52 & 51586.2\end{array}$

$\begin{array}{llllll}195 & 154257 & 3334 & 66 & 53 & 51854.6\end{array}$

$\begin{array}{llllll}195 & 154309 & 3336 & 66 & 54 & ? 52471.8\end{array}$

$\begin{array}{llllll}195 & 154323 & 3338 & 66 & 55 & ? 53400.2\end{array}$

$\begin{array}{llllll}195 & 154336 & 3340 & 66 & 56 & ? 53895.2\end{array}$

$\begin{array}{llllll}195 & 154351 & 3342 & 66 & 57 & ? 53930\end{array}$

$\begin{array}{llllll}195 & 154405 & 3344 & 66 & 58 & ? 53365.6\end{array}$

$\begin{array}{llllll}195 & 154418 & 3346 & 66 & 59 & ? 53022\end{array}$

$\begin{array}{llllll}195 & 154433 & 3348 & 66 & 60 & 52516.8\end{array}$

$\begin{array}{llllll}195 & 154445 & 3350 & 66 & 61 & 52194.2\end{array}$

$\begin{array}{llllll}195 & 154501 & 3352 & 66 & 62 & 52004.4\end{array}$

$\begin{array}{llllll}195 & 154513 & 3354 & 66 & 63 & 51905.0\end{array}$
51718.78 .1

$51719.4 \quad 8.2$

$51718.6 \quad 8.2$

51712.28 .2

51696.78 .3

$51666.5 \quad 8.3$

$51589.3 \quad 8.3$

$51392.8 \quad 8.4$

50932.28 .4

$49863 \quad 8.4$

58592.78 .5

$48051.3 \quad 8.5$

$49331.6 \quad 8.6$

506318.6

51257.18 .7

$51467.5 \quad 8.7$

51600.78 .7

51595.28 .8

$51515.8 \quad 8.8$

$51434.2 \quad 8.8$

51406.98 .9

51560.98 .9

$51579.2 \quad 9.0$

$51310.8 \quad 9.0$

$51123.6 \quad 9.0$

$51192.5 \quad 9.1$

$51486.3 \quad 9.1$

$51836.5 \quad 9.1$

$52047.4 \quad 9.2$

$52262.8 \quad 9.2$

$52462.4 \quad 9.2$

$\begin{array}{lll}52591.3 & 9.3\end{array}$

$52554.3 \quad 9.3$

$52336.7 \quad 9.3$

52016.49 .4

$51782 \quad 9.4$

$51638.8 \quad 9.4$

$51576.7 \quad 9.5$

51845.19 .5

$52462.3 \quad 9.5$

53390.69 .6

$53885.6 \quad 9.6$

53920.49 .6

$53355.9 \quad 9.7$

$53012.3 \quad 9.7$

52507.19 .7

$52184.4 \quad 9.8$

$51994.6 \quad 9.8$

51895.29 .8
BOTTOM FIELD (nT)

GRAD.

(Uncorr.) (Corr.) (Diff.) (nT/m)

$\begin{array}{llll}51727.4 & 51718.9 & 8.5 & 1.0\end{array}$

$\begin{array}{llll}51736.2 & 51727.7 & 8.5 & 14.1\end{array}$

$\begin{array}{llll}51739.8 & 51731.3 & 8.5 & 21.3\end{array}$

$\begin{array}{llll}51747.0 & 51738.5 & 8.5 & 43.6\end{array}$

$\begin{array}{llll}51746.6 & 51738.0 & 8.6 & 68.2\end{array}$

$\begin{array}{llll}51748.2 & 51739.6 & 8.6 & 120.3\end{array}$

$\begin{array}{lllll}51723.2 & 51714.6 & 8.6 & 205.9\end{array}$

$\begin{array}{llll}251596.4 & 51587.7 & 8.7 & 320.0\end{array}$

$\begin{array}{llll}251128.8 & 51120.1 & 8.7 & 308.5\end{array}$

$\begin{array}{llll}? 49533.4 & 49524.7 & 8.7 & -554.1\end{array}$

$\begin{array}{lllll}258588 & 58579.2 & 8.8 & -21.6\end{array}$

$\begin{array}{llll}? 58610.4 & 58601.6 & 8.8 & 17296.1\end{array}$

$\begin{array}{lllll}\mathbf{2} 52037.8 & 52028.9 & 8.9 & 4422.3\end{array}$

$\begin{array}{llll}750271 & 50262.1 & 8.9 & -604.3\end{array}$

$\begin{array}{llll}251080 & 51071.0 & 9.0 & -304.6\end{array}$

$\begin{array}{llll}251323.6 & 51314.6 & 9.0 & -250.2\end{array}$

$\begin{array}{llll}51611.8 & 51602.8 & 9.0 & 3.9\end{array}$

$\begin{array}{llll}51579.8 & 51570.7 & 9.1 & -39.7\end{array}$

$\begin{array}{llll}51478.8 & 51469.7 & 9.1 & -75.1\end{array}$

$\begin{array}{llll}51356.0 & 51346.9 & 9.1 & -142.6\end{array}$

$\begin{array}{llll}\text { ?51224.6 } & 51215.5 & 9.1 & -313.4\end{array}$

$\begin{array}{llll}251981.4 & 51972.2 & 9.2 & 674.8\end{array}$

$\begin{array}{llll}352045.6 & 52036.4 & 9.2 & 749.8\end{array}$

$\begin{array}{llll}? 51117.4 & 51108.1 & 9.3 & -331.8\end{array}$

$\begin{array}{llll}250735.4 & 50726.1 & 9.3 & -651.1\end{array}$

$\begin{array}{llll}250847.8 & 50838.5 & 9.3 & -580.0\end{array}$

$\begin{array}{llll}251319.6 & 51310.3 & 9.3 & -288.2\end{array}$

$\begin{array}{llll}251837 & 51827.6 & 9.4 & -14.1\end{array}$

$\begin{array}{llll}251940.8 & 51931.4 & 9.4 & -189.8\end{array}$

$\begin{array}{llll}352109.2 & 52099.8 & 9.4 & -266.9\end{array}$

$\begin{array}{llll}52433.2 & 52423.7 & 9.5 & -63.0\end{array}$

$\begin{array}{llll}52764.8 & 52755.3 & 9.5 & 269.2\end{array}$

$\begin{array}{llll}\mathbf{3} 52803.4 & 52793.9 & 9.5 & 393.1\end{array}$

$\begin{array}{llll}52452.0 & 52442.4 & 9.6 & 173.8\end{array}$

$\begin{array}{llll}51958.0 & 51948.4 & 9.6 & -111.1\end{array}$

$\begin{array}{llll}251617.6 & 51608.0 & 9.6 & -284.9\end{array}$

$\begin{array}{llll}\text { ?51636.4 } & 51626.7 & 9.7 & -19.3\end{array}$

$\begin{array}{llll}251404.2 & 51394.5 & 9.7 & -298.4\end{array}$

$\begin{array}{llll}? 51759.4 & 51749.7 & 9.7 & -156.1\end{array}$

$\begin{array}{llll}? 53136.6 & 53126.9 & 9.7 & 1089.8\end{array}$

$\begin{array}{llll}\mathbf{2 5 5 1 4 6 . 4} & 55136.6 & 9.8 & 2862.6\end{array}$

$\begin{array}{llll}255594.4 & 55584.6 & 9.8 & 2785.6\end{array}$

$\begin{array}{llll}255177.4 & 55167.6 & 9.8 & 2044.9\end{array}$

$\begin{array}{llll}\text { ?54530.6 } & 54520.7 & 9.9 & 1909.8\end{array}$

$\begin{array}{llll}\text { ?53234.2 } & 53224.3 & 9.9 & 347.9\end{array}$

$\begin{array}{llll}52461.8 & 52451.9 & 9.9 & -90.2\end{array}$

$\begin{array}{llll}52111.4 & 52101.4 & 10.0 & -135.7\end{array}$

$\begin{array}{llll}51937.8 & 51927.8 & 10.0 & -109.2\end{array}$

$\begin{array}{llll}51851.4 & 51841.4 & 10.0 & -87.9\end{array}$ 
TOP FIELD (nT)

$\begin{array}{ccccccccc}\text { LINE DAY } & \text { TIME } & \text { STA. } & X & Y & \text { (Uncorr.) } & \text { (Corr.) } & \text { (Diff.) } \\ 23 & 195 & 154526 & 3356 & 66 & 64 & 51848.0 & 51838.1 & 9.9\end{array}$

$\begin{array}{llllll}23 & 195 & 154539 & 3358 & 66 & 65\end{array}$

$\begin{array}{llllll}23 & 195 & 154550 & 3360 & 66 & 66\end{array}$

$\begin{array}{llllll}23 & 195 & 154602 & 3362 & 66 & 67\end{array}$

$\begin{array}{llllll}23 & 195 & 154614 & 3364 & 66 & 68\end{array}$

$\begin{array}{llllll}24 & 195 & 155510 & 3368 & 69 & 80\end{array}$

$\begin{array}{llllll}24 & 195 & 155526 & 3370 & 69 & 79\end{array}$

$\begin{array}{llllll}24 & 195 & 155540 & 3372 & 69 & 78\end{array}$

$\begin{array}{lllllll}24 & 195 & 155555 & 3374 & 69 & 77\end{array}$

$\begin{array}{llllll}24 & 195 & 155609 & 3376 & 69 & 76\end{array}$

24

24

\section{4}

\section{4}

24

$$
24
$$

\section{4}

$$
24
$$

\section{4}

\section{4}

24

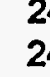

\section{4}

\section{${ }_{22}^{2}$}

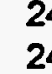

24

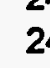

\section{4.}

\section{4}

\section{4}

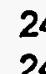

21

\section{4}

$\begin{array}{lllllll}24 & 195 & 160852 & 3446 & 69 & 41 & 51539.6\end{array}$

$\begin{array}{llllllll}24 & 195 & 160910 & 3450 & 69 & 39 & ? 51159.8\end{array}$

$\begin{array}{lllllll}24 & 195 & 160923 & 3452 & 69 & 38 & ? 51220.6\end{array}$ $\begin{array}{lllllll}24 & 195 & 160936 & 3454 & 69 & 37 & 51380.2\end{array}$
$51799.3 \quad 9.9$

$51773.3 \quad 9.9$

$\begin{array}{ll}51769 & 10.0\end{array}$

$\begin{array}{ll}51806 & 10.0\end{array}$

51717.211 .4

51717.411 .4

$51706.3 \quad 11.5$

51683.911 .5

51651.111 .5

51597.712 .3

$51446.5 \quad 12.3$

50891.412 .4

51193.612 .4

$51514 \quad 12.4$

$51634.3 \quad 12.5$

51691.912 .5

51720.312 .5

$51736.6 \quad 12.6$

$51752 \quad 12.6$

51770.612 .6

$51791.7 \quad 12.7$

$\begin{array}{lll}51822.7 & 12.7\end{array}$

$\begin{array}{lll}51874.7 & 12.7\end{array}$

$51932.6 \quad 12.8$

$\begin{array}{lll}52028.4 \quad 12.8 & \end{array}$

$\begin{array}{lll}52165 & 12.8\end{array}$

52299.312 .9

$52385.5 \quad 12.9$

52311.312 .9

52044.813 .0

51728.213 .0

51455.613 .0

51390.513 .1

51561.513 .1

$51842 \quad 13.2$

52200.213 .2

$52614.6 \quad 13.2$

53045.913 .3

53418.513 .3

53491.513 .3

52772.613 .4

52006.213 .4

51639.813 .4

51526.113 .5

51398.313 .5

51146.313 .5

$51207 \quad 13.6$

51366.613 .6
BOTTOM FIELD (nT)

GRAD.

(Uncorr.) (Corr.) (Diff.) (nT/m) $\begin{array}{llll}51803.4 & 51793.3 & 10.1 & -73.1\end{array}$

$\begin{array}{llll}51768.2 & 51758.1 & 10.1 & -67.2\end{array}$

$\begin{array}{llll}51742.0 & 51731.9 & 10.1 & -67.5\end{array}$

$\begin{array}{llll}51728.0 & 51717.8 & 10.2 & -83.6\end{array}$

$\begin{array}{llll}51831.2 & 51821.0 & 10.2 & 24.9\end{array}$

$\begin{array}{llll}51731.2 & 51719.7 & 11.5 & 4.3\end{array}$

$\begin{array}{llll}51723.2 & 51711.7 & 11.5 & -9.2\end{array}$

$\begin{array}{llll}51706.0 & 51694.5 & 11.5 & -19.3\end{array}$

$\begin{array}{llll}51673.6 & 51662.0 & 11.6 & -35.7\end{array}$

$\begin{array}{llll}51627.2 & 51615.6 & 11.6 & -58.0\end{array}$

$\begin{array}{llll}51523.4 & 51511.1 & 12.3 & -142.0\end{array}$

$\begin{array}{llll}51308.0 & 51295.7 & 12.3 & -247.2\end{array}$

?50837.6 $50825.2 \quad 12.4 \quad-108.5$

$\begin{array}{llll}51303.4 & 51291.0 & 12.4 & 159.7\end{array}$

$\begin{array}{llll}51556.6 & 51544.2 & 12.4 & 49.5\end{array}$

$\begin{array}{llll}51653.6 & 51641.1 & 12.5 & 11.1\end{array}$

$\begin{array}{llll}51699.4 & 51686.9 & 12.5 & -8.2\end{array}$

$\begin{array}{llll}51720.0 & 51707.5 & 12.5 & -21.0\end{array}$

$\begin{array}{llll}51732.8 & 51720.2 & 12.6 & -26.9\end{array}$

$\begin{array}{llll}51744.0 & 51731.4 & 12.6 & -33.8\end{array}$

$\begin{array}{llll}51757.2 & 51744.6 & 12.6 & -42.6\end{array}$

$\begin{array}{llll}51772.6 & 51759.9 & 12.7 & -52.1\end{array}$

$\begin{array}{llll}51790.0 & 51777.3 & 12.7 & -74.4\end{array}$

$\begin{array}{llll}51816.2 & 51803.5 & 12.7 & -116.7\end{array}$

$\begin{array}{llll}51880.6 & 51867.8 & 12.8 & -106.2\end{array}$

$\begin{array}{llll}51961.8 & 51949.0 & 12.8 & -130.2\end{array}$

$\begin{array}{llll}52101.4 & 52088.6 & 12.8 & -125.2\end{array}$

$\begin{array}{lllll}252260.8 & 52248.0 & 12.8 & -84.3\end{array}$

$\begin{array}{llll}? 52350 & 52337.1 & 12.9 & -79.3\end{array}$

$\begin{array}{llll}? 52238.4 & 52225.5 & 12.9 & -140.7\end{array}$

$\begin{array}{llll}251847.2 & 51834.3 & 12.9 & -345.2\end{array}$

$\begin{array}{lllll}351525.8 & 51512.8 & 13.0 & -353.1\end{array}$

$\begin{array}{lllll}2 & 51162.4 & 51149.4 & 13.0 & -502.0\end{array}$

$\begin{array}{llll}351184 & 51171.0 & 13.0 & -360.0\end{array}$

$\begin{array}{llll}51484.2 & 51471.1 & 13.1 & -148.2\end{array}$

$\begin{array}{llll}51799.2 & 51786.1 & 13.1 & -91.8\end{array}$

$\begin{array}{llll}52263.6 & 52250.5 & 13.1 & 82.3\end{array}$

$\begin{array}{llll}? 52880 & 52866.8 & 13.2 & 413.4\end{array}$

$\begin{array}{llll}253550.4 & 53537.2 & 13.2 & 805.3\end{array}$

$\begin{array}{llll}254602.6 & 54589.4 & 13.2 & 1919.3\end{array}$

$\begin{array}{llll}? 55401.4 & 55388.1 & 13.3 & 3109.2\end{array}$

$\begin{array}{llll}253205.4 & 53192.1 & 13.3 & 687.5\end{array}$

$\begin{array}{llll}\mathbf{2 5 1 4 2 2} .8 & 51409.5 & 13.3 & -978.4\end{array}$

$\begin{array}{lllll}? 51223 & 51209.6 & 13.4 & -705.2\end{array}$

$\begin{array}{llll}? 51321.4 & 51308.0 & 13.4 & -357.7\end{array}$

$\begin{array}{llll}? 51072.2 & 51058.8 & 13.4 & -556.7\end{array}$

$\begin{array}{lllll}? 16711.2 & 16697.7 & 13.5 & -56473.1\end{array}$

$\begin{array}{llll}? 50925.6 & 50912.1 & 13.5 & -483.6\end{array}$

$\begin{array}{llll}251515.6 & 51502.1 & 13.5 & 222.0\end{array}$ 
TOP FIELD (nT)

LINE DAY TIME STA. $X Y$ (Uncorr.)

24

24

24

\section{4}

\section{4}

\section{4}

24

\section{4}

24

24

24

24

24

\section{4}

24

24

\section{4}

\section{4}

\section{4}

24

\section{4}

$$
\begin{aligned}
& 25 \\
& 25
\end{aligned}
$$

25
${ }_{25}^{2}$

25

\subsection{4 .7}

52211.114 .7

52272.414 .8

51429.414 .8

51567.714 .9

51604.514 .9

51588.514 .9

$51565 \quad 15.0$

51515.818 .4

51597.418 .4

51634.318 .3

$51650.7 \quad 18.3$

$\begin{array}{ll}51662 & 18.2\end{array}$

$51676.8 \quad 18.2$

51694.618 .2

51706.718 .1

51710.918 .1

51713.818 .0

51716.218 .0

51718.218 .0

BOTTOM FIELD (nT)

GRAD.

(Uncorr.) (Corr.) (Diff.) (nT/m) $\begin{array}{lllll}51342.0 & 51328.4 & 13.6 & -101.6\end{array}$ $\begin{array}{llll}51312.8 & 51299.2 & 13.6 & -210.5\end{array}$ $\begin{array}{lllll}51502.6 & 51489.0 & 13.6 & -132.8\end{array}$ $\begin{array}{llll}51747.8 & 51734.2 & 13.6 & -44.3\end{array}$ $\begin{array}{llll}52071.8 & 52058.1 & 13.7 & 0.0\end{array}$ $\begin{array}{llll}252663.2 & 52649.5 & 13.7 & 172.5\end{array}$ $\begin{array}{lllll}253910.6 & 53896.9 & 13.7 & 918.4\end{array}$ $\begin{array}{llll}? 58534.2 & 58520.4 & 13.8 & 6334.8\end{array}$ $\begin{array}{llll}258602 & 58588.2 & 13.8 & 414.8\end{array}$

$\begin{array}{llll}258625.2 & 58611.4 & 13.8 & 15.1\end{array}$

$\begin{array}{llll}358549 & 58535.1 & 13.9 & 10768.5\end{array}$

$\begin{array}{lllll}250456.4 & 50442.5 & 13.9 & -868.5\end{array}$

$\begin{array}{lllll}350802.8 & 50788.8 & 14.0 & -322.0\end{array}$

$\begin{array}{llll}51212.2 & 51198.1 & 14.1 & 0.3\end{array}$

$\begin{array}{llll}51445.0 & 51430.9 & 14.1 & 70.5\end{array}$

$\begin{array}{llll}51578.2 & 51564.1 & 14.1 & 71.8\end{array}$

$\begin{array}{llll}51656.4 & 51642.2 & 14.2 & 64.9\end{array}$

$\begin{array}{llll}51705.8 & 51691.6 & 14.2 & 68.9\end{array}$

$\begin{array}{llll}51731.4 & 51717.2 & 14.2 & 61.3\end{array}$

$\begin{array}{llll}51734.0 & 51719.8 & 14.2 & 39.7\end{array}$

$\begin{array}{llll}51751.0 & 51736.7 & 14.3 & 47.9\end{array}$

$\begin{array}{llll}51729.0 & 51714.7 & 14.3 & 12.8\end{array}$

$\begin{array}{llll}51724.4 & 51710.1 & 14.3 & 7.9\end{array}$

$\begin{array}{llll}51727.2 & 51712.8 & 14.4 & 9.5\end{array}$

$\begin{array}{llll}51728.2 & 51713.8 & 14.4 & 11.8\end{array}$

$\begin{array}{llll}51725.4 & 51711.0 & 14.4 & 6.9\end{array}$

$\begin{array}{llll}51722.8 & 51708.4 & 14.4 & 3.0\end{array}$

$\begin{array}{llll}51720.2 & 51705.7 & 14.5 & -4.6\end{array}$

$\begin{array}{llll}51720.8 & 51706.3 & 14.5 & -22.3\end{array}$

$\begin{array}{llll}51752.2 & 51737.7 & 14.5 & -74.8\end{array}$

$\begin{array}{llll}252019 & 52004.4 & 14.6 & -339.0\end{array}$

$\begin{array}{lllll}258595.4 & 58580.8 & 14.6 & 10341.3\end{array}$

$\begin{array}{lllll}\mathbf{3} 50955.4 & 50940.8 & 14.6 & -801.3\end{array}$

$\begin{array}{lllll}51582.8 & 51568.1 & 14.7 & 0.3\end{array}$

$\begin{array}{llll}51619.0 & 51604.3 & 14.7 & -0.7\end{array}$

$\begin{array}{lllll}51606.0 & 51591.3 & 14.7 & 4.3\end{array}$

$\begin{array}{llll}51570.6 & 51555.8 & 14.8 & -15.4\end{array}$

$\begin{array}{lllll}51453.2 & 51432.2 & 21.0 & -132.8\end{array}$

$\begin{array}{llll}51577.8 & 51556.9 & 20.9 & -62.3\end{array}$

$\begin{array}{llll}51628.4 & 51607.6 & 20.8 & -39.7\end{array}$

$\begin{array}{llll}51649.4 & 51628.6 & 20.8 & -32.1\end{array}$

$\begin{array}{llll}51661.4 & 51640.7 & 20.7 & -30.8\end{array}$

$\begin{array}{llll}51675.0 & 51654.3 & 20.7 & -32.8\end{array}$

$\begin{array}{llll}51693.8 & 51673.2 & 20.6 & -31.1\end{array}$

$\begin{array}{llll}51707.4 & 51686.8 & 20.6 & -28.5\end{array}$

$\begin{array}{llll}51718.8 & 51698.3 & 20.5 & -16.7\end{array}$

$\begin{array}{llll}51723.0 & 51702.5 & 20.5 & -14.4\end{array}$

$\begin{array}{llll}51727.4 & 51707.0 & 20.4 & -11.1\end{array}$

$\begin{array}{llll}51734.6 & 51714.2 & 20.4 & -2.6\end{array}$ 
TOP FIELD (nT)

$\begin{array}{cccccccc}\text { LINE DAY } & \text { TIME } & \text { STA. } & \mathbf{X} & \mathbf{Y} & \text { (Uncorr.) } & \text { (Corr.) } \\ 25 & 196 & 90752 & 26 & 72 & 12 & 51736.4 & 51718.5\end{array}$

25

25

25

25

25

25

25

25

25

25

25

25

25

25

25

25

25

25

25

25

25

25

25

25

25

25

25

25

25

25

25

25

25

25

25

25

25

25

25

25

25

25

25

25

25

25

25

25 $\begin{array}{ll}196 & 90805 \\ 19620\end{array}$

19690833

19690846

19690900

19690916

19690930

19690947

$196 \quad 91012$

19691026

19691041

19691055

19691109

$196 \quad 91121$

19691135

19691149

19691203

19691218

19691230

19691244

$\begin{array}{ll}196 & 91258\end{array}$

$196 \quad 91312$

$196 \quad 91326$

$196 \quad 91341$

19691356

$196 \quad 91410$

$196 \quad 91425$

19691442

$196 \quad 91456$

$196 \quad 91510$

$196 \quad 91535$

19691548

19691602

19691616

$196 \quad 91629$

19691643

19691657

$196 \quad 91711$

$196 \quad 91725$

19691744

19691802

19691826

19691845

19691901

19691914

$\begin{array}{ll}196 & 91927\end{array}$

19691940

19691956 $\begin{array}{llll}28 & 72 & 13 & 517\end{array}$

$\begin{array}{lll}30 & 72 & 14\end{array}$

$\begin{array}{lll}32 & 72 & 15\end{array}$

$\begin{array}{lll}34 & 72 & 16\end{array}$

$\begin{array}{lll}36 & 72 & 17\end{array}$

$\begin{array}{lll}38 & 72 & 18\end{array}$

$\begin{array}{lll}40 & 72 & 19\end{array}$

$42 \quad 72 \quad 20$

$\begin{array}{lll}44 & 72 & 21\end{array}$

$\begin{array}{lll}46 & 72 \quad 22\end{array}$

$\begin{array}{lll}48 & 72 & 23\end{array}$

$\begin{array}{lll}50 & 72 & 24\end{array}$

$\begin{array}{lll}52 & 72 & 25\end{array}$

$\begin{array}{lll}54 & 72 & 26\end{array}$

$\begin{array}{llll}56 & 72 & 27 & ? 52415.6\end{array}$

$\begin{array}{llll}58 & 72 & 28 & ? 52843.8\end{array}$

$\begin{array}{llll}60 & 72 & 29 & \text { ?52897.6 }\end{array}$

$\begin{array}{llll}62 & 72 & 30 & 52585.4\end{array}$

$\begin{array}{llll}64 & 72 & 31 & 52241.6\end{array}$

$\begin{array}{llll}66 & 72 & 32 & 51964.6\end{array}$

$\begin{array}{llll}68 & 72 & 33 & 51762.4\end{array}$

$\begin{array}{llll}70 & 72 & 34 & 51599.0\end{array}$

$\begin{array}{llll}72 & 72 & 35 & 51465.4\end{array}$

$\begin{array}{llll}74 & 72 & 36 & 51322.6\end{array}$

$\begin{array}{llll}76 & 72 & 37 & ? 51245.8\end{array}$

$\begin{array}{llll}78 & 72 & 38 & ? 51326.2\end{array}$

$\begin{array}{llll}80 & 72 & 39 & 51661.2\end{array}$

$\begin{array}{llll}82 & 72 & 40 & ? 52096.4\end{array}$

84

$\begin{array}{llll}72 & 41 & 52154.6\end{array}$

$\begin{array}{lll}72 & 42 & 52017.6\end{array}$

$\begin{array}{llll}88 & 72 & 43 & 52051.0\end{array}$

$\begin{array}{llll}90 & 72 & 44 & 52222.6\end{array}$

$\begin{array}{llll}92 & 72 & 45 & 52391.2\end{array}$

$\begin{array}{llll}94 & 72 & 46 & 52572.2\end{array}$

$\begin{array}{llll}96 & 72 & 47 & ? 52699\end{array}$

$\begin{array}{llll}98 & 72 & 48 & ? 52604.2\end{array}$

$\begin{array}{llll}100 & 72 & 49 & ? 52266.2\end{array}$

$\begin{array}{llll}102 & 72 & 50 & 51789.0\end{array}$

$\begin{array}{llll}104 & 72 & 51 & ? 51151.6\end{array}$

$\begin{array}{llll}106 & 72 & 52 & ? 50319.2\end{array}$

$\begin{array}{llll}108 & 72 & 53 & ? 50077\end{array}$

$\begin{array}{llll}110 & 72 & 54 & ? 51093\end{array}$

$\begin{array}{llll}112 & 72 & 55 & ? 51968.6\end{array}$

$\begin{array}{llll}114 & 72 & 56 & 52088.6\end{array}$

$\begin{array}{llll}116 & 72 & 57 & 52148.4\end{array}$

$\begin{array}{llll}118 & 72 & 58 & 52191.2\end{array}$

$\begin{array}{llll}120 & 72 & 59 & 52099.8\end{array}$

$\begin{array}{llll}122 & 72 & 60 & 51936.4\end{array}$
51718.517 .9

$51718.7 \quad 17.9$

$51716.4 \quad 17.8$

$51713.4 \quad 17.8$

$51710.6 \quad 17.8$

$51703.9 \quad 17.7$

$51690.5 \quad 17.7$

$51651.4 \quad 17.6$

51638.817 .6

$51605.3 \quad 17.5$

$51559.1 \quad 17.5$

$51530.8 \quad 17.4$

$51540.2 \quad 17.4$

$51638.5 \quad 17.3$

$51919.5 \quad 17.3$

$52398.3 \quad 17.3$

$\begin{array}{lll}52826.6 & 17.2\end{array}$

$52880.4 \quad 17.2$

52568.217 .2

$52224.5 \quad 17.1$

$51947.5 \quad 17.1$

$51745.4 \quad 17.0$

$51582 \quad 17.0$

51448.417 .0

$51305.7 \quad 16.9$

$51228.9 \quad 16.9$

$51309.4 \quad 16.8$

$51644.4 \quad 16.8$

$52079.7 \quad 16.7$

$52137.9 \quad 16.7$

$52000.9 \quad 16.7$

$52034.4 \quad 16.6$

$52206 \quad 16.6$

$52374.7 \quad 16.5$

52555.716 .5

52682.616 .4

52587.816 .4

52249.816 .4

51772.716 .3

51135.316 .3

$50303 \quad 16.2$

$50060.8 \quad 16.2$

51076.916 .1

51952.616 .0

52072.616 .0

52132.416 .0

52175.315 .9

$52083.9 \quad 15.9$

$51920.6 \quad 15.8$
BOTTOM FIELD (nT)

(Uncorr.) (Corr.) (Diff.)

$\begin{array}{lll}51736.2 & 51715.8 & 20.4\end{array}$

$\begin{array}{llll}51736.4 & 51716.1 & 20.3\end{array}$

$\begin{array}{lll}51731.6 & 51711.3 & 20.3\end{array}$

$\begin{array}{llll}51728.6 & 51708.4 & 20.2\end{array}$

51736.2

51732.8

51717.6

51697.6

51669.2

51631.0

51572.8

51520.4

51483.4

51529.8

$? 51775$

?52322.8

?52915.4

53011.4

?52629.6

?52235.8

51927.8

51715.8

51504.8

51390.8

?51094.6

?50883.4

?50893.4

$? 51432$

$? 52567.4$

$? 52409.4$

?51908.6

51940.4

52250.4

52360.4

$? 52736$

?52991.2

?52860.2

$? 52505.4$

51888.6

?51061.4

?52914.6

?47997.8

$? 58602$

?58602.2

?52104

?52327.8

?52160.8

?51987.6

?51720.4

$51716.0 \quad 20.2$

$51712.7 \quad 20.1$

$51697.5 \quad 20.1$

$51677.6 \quad 20.0$

$51649.2 \quad 20.0$

$51611.1 \quad 19.9$

$51553.0 \quad 19.8$

$51500.6 \quad 19.8$

$51463.7 \quad 19.7$

$51510.1 \quad 19.7$

51755.3

52303.2

52895.8

52991.9

52610.1

52216.4

51908.4

51696.5

51485.5

51371.6

51075.4

50864.3

50874.3

51413.0

52548.4

52390.5

51889.7

51921.6

52231.6

52341.7

52717.3

52972.6

52841.6

52486.9

51870.1

51043.0

52896.2 
TOP FIELD (nT)

$\begin{array}{ccccccc}\text { LINE } & \text { DAY } & \text { TIME } & \text { STA. } & \text { X } & \text { Y } & \text { (Uncorr.) } \\ 25 & 196 & 92009 & 124 & 72 & 61 & 51866.2 \\ 25 & 196 & 92024 & 126 & 72 & 62 & 51834.8 \\ 25 & 196 & 92037 & 128 & 72 & 63 & 51810.6 \\ 25 & 196 & 92049 & 130 & 72 & 64 & 51794.2 \\ 25 & 196 & 92101 & 132 & 72 & 65 & 51780.8 \\ 25 & 196 & 92113 & 134 & 72 & 66 & 51768.6 \\ 25 & 196 & 92126 & 136 & 72 & 67 & 51754.2 \\ 25 & 196 & 92139 & 138 & 72 & 68 & 51730.6 \\ 25 & 196 & 92153 & 140 & 72 & 69 & 51694.6 \\ 25 & 196 & 92207 & 142 & 72 & 70 & 51629.6 \\ 25 & 196 & 92220 & 144 & 72 & 71 & 51493.2 \\ 25 & 196 & 92233 & 146 & 72 & 72 & ? 51099.6 \\ 25 & 196 & 92249 & 148 & 72 & 73 & ? 50158.2 \\ 25 & 196 & 92307 & 150 & 72 & 74 & ? 51123.6 \\ 25 & 196 & 92320 & 152 & 72 & 75 & ? 51601.2 \\ 25 & 196 & 92334 & 154 & 72 & 76 & 51656.0 \\ 25 & 196 & 92347 & 156 & 72 & 77 & 51698.4 \\ 25 & 196 & 92402 & 158 & 72 & 78 & 51726.2 \\ 26 & 196 & 92857 & 160 & 75 & 78 & 51710.4 \\ 26 & 196 & 92914 & 162 & 75 & 77 & 51678.6 \\ 26 & 196 & 92928 & 164 & 75 & 76 & 51622.4 \\ 26 & 196 & 92941 & 166 & 75 & 75 & ? 51514.8 \\ 26 & 196 & 92957 & 168 & 75 & 74 & ? 51441 \\ 26 & 196 & 93012 & 170 & 75 & 73 & ? 50860 \\ 26 & 196 & 93031 & 172 & 75 & 72 & ? 51136.8 \\ 26 & 196 & 93046 & 174 & 75 & 71 & 51505.6 \\ 26 & 196 & 93058 & 176 & 75 & 70 & 51637.2 \\ 26 & 196 & 93112 & 178 & 75 & 69 & 51700.8 \\ 26 & 196 & 93124 & 180 & 75 & 68 & 51736.0 \\ 26 & 196 & 93136 & 182 & 75 & 67 & 51759.8 \\ 26 & 196 & 93151 & 184 & 75 & 66 & 51779.6 \\ 26 & 196 & 93203 & 186 & 75 & 65 & 51800.4 \\ 26 & 196 & 93220 & 188 & 75 & 64 & 51831.4 \\ 26 & 196 & 93232 & 190 & 75 & 63 & 51884.8 \\ 26 & 196 & 93244 & 192 & 75 & 62 & 51970.6 \\ 26 & 196 & 93257 & 194 & 75 & 61 & 52132.6 \\ 26 & 196 & 93309 & 196 & 75 & 60 & 52473.4 \\ 26 & 196 & 93323 & 198 & 75 & 59 & ? 53018 \\ 26 & 196 & 93337 & 200 & 75 & 58 & ? 53480.4 \\ 26 & 196 & 93356 & 202 & 75 & 57 & ? 52908.8 \\ 26 & 196 & 93410 & 204 & 75 & 56 & ? 52080.6 \\ 26 & 196 & 93423 & 206 & 75 & 55 & ? 51373.2 \\ 26 & 196 & 93437 & 208 & 75 & 54 & ? 50875.4 \\ 26 & 196 & 93451 & 210 & 75 & 53 & ? 50598 \\ 26 & 196 & 93505 & 212 & 75 & 52 & ? 50657.6 \\ 26 & 196 & 93520 & 214 & 75 & 51 & ? 50998.8 \\ 26 & 196 & 93533 & 216 & 75 & 50 & 51405.2 \\ 26 & 196 & 93545 & 218 & 75 & 49 & 51739.2 \\ 26 & 196 & 93558 & 220 & 75 & 48 & 51974.8\end{array}$

BOTTOM FIELD (nT)

GRAD.

(Corr.) (Diff.) (Uncorr.) (Corr.) (Diff.) (nT/m) $\begin{array}{llllll}51850.4 & 15.8 & 51738.8 & 51720.9 & 17.9 & -208.9\end{array}$ $51819 \quad 15.8$ $51794.9 \quad 15.7$ $51778.5 \quad 15.7$ 51765.115 .7 $51753 \quad 15.6$ $51738.6 \quad 15.6$ 51715.115 .5 51679.115 .5 51614.115 .5 $51477.8 \quad 15.4$ $51084.2 \quad 15.4$ 50142.915 .3 $51108.3 \quad 15.3$ $51585.9 \quad 15.3$ $51640.8 \quad 15.2$ $51683.2 \quad 15.2$ $51711.1 \quad 15.1$ $51696.1 \quad 14.3$ $51664.4 \quad 14.2$ 51608.214 .2 $51500.6 \quad 14.2$ $51426.9 \quad 14.1$ $50845.9 \quad 14.1$ $51122.8 \quad 14.0$ $51491.6 \quad 14.0$ $51623.3 \quad 13.9$ $51686.9 \quad 13.9$ $51722.1 \quad 13.9$ $51746 \quad 13.8$ $\begin{array}{lll}51765.8 & 13.8\end{array}$ $51786.6 \quad 13.8$ $51817.7 \quad 13.7$ $51871.1 \quad 13.7$ $51957 \quad 13.6$ $52119 \quad 13.6$ $52459.8 \quad 13.6$ $53004.5 \quad 13.5$ $53466.9 \quad 13.5$ 52895.413 .4 52067.213 .4 51359.813 .4 $50862.1 \quad 13.3$ $50584.7 \quad 13.3$ $50644.4 \quad 13.2$ $50985.6 \quad 13.2$ $51392 \quad 13.2$ $51726.1 \quad 13.1$ $51961.7 \quad 13.1$
51755.2

51756.0

51749.0

51744.4

51737.8

51729.6

51713.4

51686.0

51632.4

51502.0

?51004.6

?49592.2

?51266.4

51393.0

51576.2

51660.0

51701.6

51702.2

51657.0

51582.4

51367.0

?50483.2

?49661.4

?51140.6

51502.8

51649.2

51703.4

51731.2

51748.8

51761.0

51771.8

51787.4

51823.6

51891.6

52025.0

?52444.4

$? 53398$

?18298.2

?54176.8

?51424.4

$? 50657$

?50206.6

$? 50155.4$

?50027.8

?50731.8

51371.0

51787.6

52121.8
$51737.4 \quad 17.8$

$51738.2 \quad 17.8$

$51731.3 \quad 17.7$

$51726.7 \quad 17.7$

$51720.1 \quad 17.7$

$51712.0 \quad 17.6$

$51695.8 \quad 17.6$

$51668.5 \quad 17.5$

$51614.9 \quad 17.5$

$51484.6 \quad 17.4$

$50987.2 \quad 17.4$

$49574.9 \quad 17.3$

$51249.1 \quad 17.3$

$51375.8 \quad 17.2$

$51559.0 \quad 17.2$

$51642.9 \quad 17.1$

$51684.5 \quad 17.1$

$51686.1 \quad 16.1$

$51641.0 \quad 16.0$

16.0

$51351.0 \quad 16.0$

50467.3

49645.5

51124.8

15.9

15.8

51487.1

51633.5

51687.7

51715.6

51733.2

51745.5

51756.3

51772.0

51808.2

51876.3

52009.7

52429.1

53382.8

18283.0

54161.7

51409.3

50642.0

50191.6

50140.5

50012.9

50717.

51356.2

51772

52107.1
15.7

15.7

15.7

15.6

15.6

15.5

15.5

15.4

15.4

15.3

15.3

15.3

15.2

15.2

15.1

15.1

15.0

15.0

14.9

14.9

14.8

14.8

14.7

14.7
$-130.5$

$-89.5$

$-74.1$

$-59.7$

$-50.5$

$-40.3$

$-28.2$

$-14.1$

4.6

14.4

$-155.7$

$-927.9$

234.1

$-341.3$

$-130.8$

$-63.0$

$-40.3$

$-13.4$

$-35.4$

$-65.6$

$-242.3$

$-1570.2$

$-1964.9$

6.2

$-4.6$

19.7

4.3

$-7.9$

$-18.0$

$-30.5$

$-46.9$

$-72.1$

$-100.3$

$-129.5$

$-176.4$

$-47.5$

623.0

$-57675.7$

2078.7

$-1075.7$

$-1174.1$

$-1096.4$

$-725.6$

$-1032.5$

$-437.7$

$-56.1$

79.3

241.0 
TOP FIELD (nT)

BOTTOM FIELD (nT)

GRAD.

\begin{tabular}{|c|c|c|c|c|c|c|c|c|c|c|c|c|}
\hline 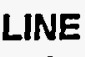 & DAY & TIME & TA. & $x$ & $\mathbf{Y}$ & corr.) & (Corr.) & (Diff.) & r.) & (Corr.) & (Diff.) & $\mathrm{nT} / \mathrm{m})$ \\
\hline 26 & 196 & 93646 & 222 & 75 & 47 & 52100.6 & 52087.6 & 13.0 & $? 52369.6$ & 52355.1 & 14.5 & 441.0 \\
\hline 26 & 196 & 93659 & 224 & 75 & 46 & 51990.4 & 51977.5 & 12.9 & $? 52143$ & 52128.5 & 14.5 & 250.2 \\
\hline 26 & 196 & 93713 & 226 & 75 & 45 & 51791.6 & 51778.7 & 12.9 & ?51607.2 & 51592.8 & 14.4 & -302.3 \\
\hline 26 & 196 & 93726 & 228 & 75 & 44 & 51711.0 & 51698.2 & 12.8 & 35.4 & 1481.0 & 14.4 & -353.4 \\
\hline 2 & 196 & 739 & 230 & 75 & 43 & 51781.6 & 51768.8 & 12.8 & 4.4 & 1680.1 & 14.3 & -143.0 \\
\hline 26 & 196 & 93752 & 232 & 75 & 42 & 51940.8 & & 12.8 & ?52102.8 & 88.5 & 14.3 & 265.6 \\
\hline 26 & 196 & 93812 & 234 & 75 & 41 & 52053.0 & 52040.3 & 12.7 & $? 52229.6$ & 5.4 & 14.2 & 289.5 \\
\hline 26 & 196 & 93826 & 236 & 75 & 40 & 52090.6 & 52077.9 & 12.7 & ?52374.2 & 52360.0 & 14.2 & 464.9 \\
\hline 26 & 196 & 93840 & 238 & 75 & 39 & 51898.6 & 51886 & 12.6 & $? 51976.4$ & 51962.3 & 14.1 & 127.5 \\
\hline 26 & 196 & 93854 & 240 & 75 & 38 & 51641.8 & 51629.2 & 12.6 & $? 51394.2$ & 51380.1 & 14.1 & -405.9 \\
\hline 26 & 196 & 93908 & 242 & 75 & 37 & 514 & 51475.7 & 12.5 & 88.4 & 4.3 & 14.1 & -491.5 \\
\hline 26 & 196 & 93921 & 244 & 75 & 36 & & 51347.9 & 12.5 & & & 14.0 & 158.0 \\
\hline 26 & 196 & 93935 & 246 & 75 & 35 & $? 51$ & 51330.1 & 12.5 & $? 50$ & 3.2 & 14.0 & -582.6 \\
\hline 26 & 196 & 93948 & 248 & 75 & 34 & 51 & 51417.4 & 12.4 & 3.8 & & 13.9 & -173.8 \\
\hline 4 & 196 & 001 & 250 & 75 & 33 & 9.4 & 17 & 12.4 & & & 10.9 & -48.2 \\
\hline 2 & 196 & 4014 & 252 & 75 & 32 & & 1.6 & 12.4 & & 9.2 & 13.8 & -50.8 \\
\hline 26 & 196 & 94026 & 254 & 75 & 31 & 516 & 51609.9 & 12.3 & & 38.0 & 13.8 & -33.4 \\
\hline 26 & 196 & 94039 & 256 & 75 & 30 & 51 & 51605.5 & 12.3 & & 7.7 & 13.7 & -26.9 \\
\hline 26 & 196 & 94051 & 258 & 75 & 29 & & 7.9 & 12.3 & & 3.5 & 13.7 & -21.3 \\
\hline 26 & 196 & 94104 & 260 & 75 & 28 & & 51517 & 12.2 & & & 13.7 & -8.9 \\
\hline 26 & 196 & 94117 & 262 & 75 & 27 & & & 12.2 & & & 13.6 & 17.7 \\
\hline 26 & 196 & 94130 & 264 & 75 & 26 & & 51 & 12.1 & & & 3.6 & 7.9 \\
\hline 26 & 196 & 94142 & 266 & 75 & 25 & & & 12.1 & & & 13.5 & 23.6 \\
\hline 26 & 196 & 94155 & 268 & 75 & 24 & & 51 & 12.1 & & & 13.0 & 167.2 \\
\hline 26 & 196 & 94208 & 270 & 75 & 23 & 51 & 51501.2 & 12.0 & & 2.0 & 13.4 & 183.9 \\
\hline 26 & 196 & 94221 & 272 & 75 & 22 & & 32 & 12.0 & & 4.6 & 13.4 & 170.5 \\
\hline 26 & 196 & 94235 & 274 & 75 & 21 & & 51 & 12.0 & & .4 & 13.4 & 147.5 \\
\hline 28 & 196 & 94248 & 276 & 75 & 20 & & 51 & 11.9 & & 3.5 & 13.3 & 117.4 \\
\hline 2 & 196 & 94301 & 278 & 75 & 19 & 51 & 51 & 11.9 & & & 13 & 92.8 \\
\hline 26 & 196 & 94314 & 280 & 75 & 18 & .2 & 1.4 & 11.8 & & & 13.2 & 2.5 \\
\hline 26 & 196 & 94327 & 282 & 75 & 17 & & & 11.8 & & & 13.2 & 54.4 \\
\hline 26 & 196 & 94345 & 284 & 75 & 16 & & & 11.8 & & & 13.1 & 43.6 \\
\hline 26 & 196 & 94402 & 286 & 75 & 15 & & & 11.7 & & & 13.1 & 35.1 \\
\hline 26 & 196 & 94415 & 288 & 75 & 14 & & 517 & 11.7 & & & 13.0 & 27.5 \\
\hline 26 & 196 & 94431 & 290 & 75 & 13 & & 517 & 11.6 & & & 13.0 & 3.0 \\
\hline 26 & 196 & 94443 & 292 & 75 & 12 & 51 & 51 & 11.6 & .6 & 8.7 & 12. & 22.0 \\
\hline 26 & 196 & 94456 & 294 & 75 & 11 & & 51 & 11.5 & & & 12.9 & 14.8 \\
\hline 26 & 196 & 94509 & 296 & 75 & 10 & & & 11.5 & & & 12.8 & 15.4 \\
\hline 26 & 196 & 94526 & 298 & 75 & 9 & & & 11.5 & & & 12.8 & 14.1 \\
\hline 26 & 196 & 94537 & 300 & 75 & 8 & & 51 & 11.4 & & & 12.7 & 8.2 \\
\hline 26 & 196 & & 302 & 75 & 7 & & & 11.4 & & & 12.7 & 6.9 \\
\hline 26 & 196 & 94601 & 304 & 75 & 6 & & 89 & 11.4 & & 0.3 & 12.7 & 4.3 \\
\hline 26 & 196 & 94629 & 306 & 75 & 5 & & 51680.1 & 11.3 & & 9.0 & 12.6 & 0.3 \\
\hline 26 & 196 & 94642 & 308 & 75 & 4 & & 51670.2 & 11.2 & & 3.5 & 12.5 & -8.9 \\
\hline 26 & 196 & 94655 & 310 & 75 & 3 & 51666.2 & 51655 & 11.2 & & 3.9 & 12.5 & -16.1 \\
\hline 26 & 196 & 94708 & 312 & 75 & 2 & 51644.2 & 51633 & 11.2 & 510 & 14.2 & 12.4 & -28.8 \\
\hline 26 & 196 & 94721 & 314 & 75 & 1 & & & 11.1 & & 51.2 & 12.4 & -60.3 \\
\hline 26 & 196 & 34 & 316 & 75 & 0 & & & 11.1 & & 3.3 & 12.3 & -164.9 \\
\hline 27 & 196 & 95119 & 320 & 78 & 0 & 51573.2 & 51562.6 & 10.6 & 51439.6 & 51427.5 & 12.1 & -219.0 \\
\hline
\end{tabular}


TOP FIELD (nT)

$\begin{array}{cccccccc}\text { LINE DAY } & \text { TIME } & \text { STA. } & \mathbf{X} & \mathbf{Y} & \text { (Uncorr.) } & \text { (Corr.) } \\ 27 & 196 & 95132 & 322 & 78 & 1 & 51619.0 & 51608.7\end{array}$

$27 \quad 196 \quad 95144$

$\begin{array}{lll}27 & 196 & 95158\end{array}$

$\begin{array}{lll}27 & 196 & 95211\end{array}$

$\begin{array}{lll}27 & 196 & 95224\end{array}$

$\begin{array}{lll}27 & 196 & 95237\end{array}$

$\begin{array}{lll}27 & 196 & 95250\end{array}$

$\begin{array}{lll}27 & 196 & 95302\end{array}$

$\begin{array}{lll}27 & 196 & 95317\end{array}$

$\begin{array}{lll}27 & 196 & 95329\end{array}$

27196

$27 \quad 196$

$27 \quad 196$

27196

$27 \quad 196$

27196

$27 \quad 196$

27196

$27 \quad 196$

27196

$27 \quad 196$

27196

27196

$27 \quad 196$

$27 \quad 196$

$27 \quad 196$

$27 \quad 196$

27196

27196

$27 \quad 196$

27196

27196

$27 \quad 196$

$27 \quad 196$

27196

$27 \quad 196$

$27 \quad 196$

$27 \quad 196$

$27 \quad 196$

27196

27

27

27

27

27

27

27

27
32478

$\begin{array}{ll}326 & 78\end{array}$

$\begin{array}{lll}328 & 78 \quad 4\end{array}$

$\begin{array}{lll}330 & 78 & 5\end{array}$

33278

$\begin{array}{lll}334 & 78 & 7\end{array}$

$\begin{array}{ll}336 & 78\end{array}$

$\begin{array}{lll}338 & 78 & 9\end{array}$

$\begin{array}{lll}340 & 78 & 10\end{array}$

$\begin{array}{lll}342 & 78 & 11\end{array}$

$\begin{array}{lll}344 & 78 & 12\end{array}$

$\begin{array}{lll}346 & 78 & 13\end{array}$

$\begin{array}{lll}348 & 78 & 14\end{array}$

$\begin{array}{lll}350 & 78 & 15\end{array}$

$\begin{array}{lll}352 & 78 & 16\end{array}$

$\begin{array}{lll}354 & 78 & 17\end{array}$

$\begin{array}{lll}356 & 78 & 18\end{array}$

$\begin{array}{lll}358 & 78 & 19\end{array}$

360

362

364

366

368

370

\section{2}

\section{4}

376

\section{8}

380

\section{2}

\section{4}

\section{6}

388

$$
390
$$

392

394

396

398

400$$
40
$$

404

406

408

410

412

414

416

418
51644.110 .3

$\begin{array}{lll}51674.4 & 51664.2 & 10.2\end{array}$

51690.2

51699.8

51708.0

51714.0

51718.2

51722.8

51725.6

51728.4

51731.8

51734.2

51737.4

51739.8

51743.2

51745.0

51680

51689.710 .1

$51697.9 \quad 10.1$

$51704 \quad 10.0$

51708.210 .0

$51712.9 \quad 9.9$

$51715.7 \quad 9.9$

$51718.6 \quad 9.8$

$51722 \quad 9.8$

$51724.4 \quad 9.8$

$51727.7 \quad 9.7$

$51730.1 \quad 9.7$

$51733.6 \quad 9.6$

$51735.5 \quad 9.5$

$51733.7 \quad 9.5$

51719.49 .4

$51675.4 \quad 9.4$

$51531.9 \quad 9.3$

$51151.5 \quad 9.3$

$50379.6 \quad 9.2$

$49300.8 \quad 9.2$

$48840.3 \quad 9.1$

$49471.5 \quad 9.1$

$50118.2 \quad 9.0$

$50777.4 \quad 9.0$

51215.18 .9

$51442.7 \quad 8.9$

$51549.4 \quad 8.8$

$51568.8 \quad 8.8$

$51529.7 \quad 8.7$

$\begin{array}{lll}51455.9 & 8.7\end{array}$

51384.28 .6

51420.68 .6

51668.78 .5

52003.18 .5

$52241.4 \quad 8.4$

$52273 \quad 8.4$

52139.28 .4

$51914.3 \quad 8.3$

$51692.1 \quad 8.3$

51547.28 .2

51527.28 .2

$51770.5 \quad 8.1$

$51950.5 \quad 8.1$

518998.0

51687.48 .0
BOTTOM FIELD (nT)

GRAD.

(Uncorr.) (Corr.) (Diff.)

(nT/m)

$\begin{array}{llll}51556.6 & 51544.9 & 11.7 & -102.3\end{array}$

$\begin{array}{llll}51618.2 & 51606.5 & 11.7 & -59.3\end{array}$

$\begin{array}{llll}51650.8 & 51639.1 & 11.7 & -38.7\end{array}$

$\begin{array}{llll}51671.8 & 51660.1 & 11.7 & \mathbf{- 3 0 . 2}\end{array}$

$\begin{array}{llll}51686.2 & 51674.6 & 11.6 & -22.3\end{array}$

$\begin{array}{llll}51696.0 & 51684.4 & 11.6 & -19.7\end{array}$

$\begin{array}{llll}51704.0 & 51692.4 & 11.6 & -16.4\end{array}$

$\begin{array}{llll}51710.8 & 51699.2 & 11.6 & -12.1\end{array}$

$\begin{array}{llll}51716.0 & 51704.5 & 11.5 & -11.1\end{array}$

$\begin{array}{llll}51721.4 & 51709.9 & 11.5 & -6.9\end{array}$

$\begin{array}{llll}51726.6 & 51715.1 & 11.5 & -3.0\end{array}$

$\begin{array}{llll}51731.4 & 51719.9 & 11.5 & -0.7\end{array}$

$\begin{array}{llll}51738.0 & 51726.6 & 11.4 & 6.2\end{array}$

$\begin{array}{llll}51744.6 & 51733.2 & 11.4 & 11.8\end{array}$

$\begin{array}{llll}51754.2 & 51742.8 & 11.4 & 23.6\end{array}$

$\begin{array}{llll}51765.0 & 51753.6 & 11.4 & 35.7\end{array}$

$\begin{array}{llll}51785.6 & 51774.3 & 11.3 & 66.6\end{array}$

$\begin{array}{llll}51807.2 & 51795.9 & 11.3 & 104.9\end{array}$

$\begin{array}{llll}51839.0 & 51827.7 & 11.3 & 180.7\end{array}$

$\begin{array}{llll}251872.6 & 51861.3 & 11.3 & 307.9\end{array}$

$\begin{array}{llll}251856.8 & 51845.6 & 11.2 & 517.4\end{array}$

$\begin{array}{llll}251743 & 51731.8 & 11.2 & 954.4\end{array}$

$\begin{array}{llll}? 51042.6 & 51031.4 & 11.2 & 1071.8\end{array}$

$\begin{array}{lllll}252055.2 & 52044.1 & 11.1 & 4500.3\end{array}$

$\begin{array}{llll}? 49004 & 48992.9 & 11.1 & 253.4\end{array}$

$\begin{array}{lllll}? 49231.2 & 49220.1 & 11.1 & -408.9\end{array}$

?50035.6 $50024.6 \quad 11.0 \quad-150.2$

?50696.6 $50685.6 \quad 11.0 \quad-147.2$

$\begin{array}{llll}51125.6 & 51114.6 & 11.0 & -161.3\end{array}$

$\begin{array}{llll}51363.4 & 51352.4 & 11.0 & -144.6\end{array}$

$\begin{array}{llll}51483.4 & 51472.4 & 11.0 & -122.6\end{array}$

$\begin{array}{llll}51506.6 & 51495.7 & 10.9 & -116.4\end{array}$

$\begin{array}{llll}51454.2 & 51443.3 & 10.9 & -138.0\end{array}$

$\begin{array}{llll}51333.6 & 51322.7 & 10.9 & -214.8\end{array}$

$\begin{array}{llll}251127 & 51116.1 & 10.9 & -435.7\end{array}$

$\begin{array}{llll}250917.8 & 50907.0 & 10.8 & -838.4\end{array}$

$\begin{array}{llll}? 51362 & 51351.2 & 10.8 & -516.7\end{array}$

$\begin{array}{llll}? 52080.2 & .52069 .4 & 10.8 & 112.5\end{array}$

$\begin{array}{lllll}? 52512.4 & 52501.6 & 10.8 & 430.5\end{array}$

$\begin{array}{llll}? 52536.8 & 52526.1 & 10.7 & 418.7\end{array}$

$\begin{array}{llll}\text { ?52344.6 } & 52333.9 & 10.7 & 323.0\end{array}$

$\begin{array}{llll}51945.4 & 51934.7 & 10.7 & 37.4\end{array}$

$\begin{array}{llll}51566.2 & 51555.5 & 10.7 & -220.0\end{array}$

$\begin{array}{llll}? 51324.4 & 51313.8 & 10.6 & -378.7\end{array}$

$\begin{array}{lllll}? 51029 & 51018.4 & 10.6 & -830.2\end{array}$

$\begin{array}{lllll}? 51571.4 & 51560.8 & 10.6 & -339.7\end{array}$

$\begin{array}{llll}52024.0 & 52013.4 & 10.6 & 107.2\end{array}$

$\begin{array}{llll}51949.6 & 51939.1 & 10.5 & 69.8\end{array}$

$\begin{array}{llll}51659.8 & 51649.3 & 10.5 & -58.4\end{array}$ 
TOP FIELD (nT)

LINE DAY TIME STA.

$$
27
$$

27

\section{7}

27

27

\section{7}

\section{7}

\begin{abstract}
$X Y$ (Uncorr.) (Corr.)
\end{abstract}
$\begin{array}{lll}78 & 50 & 51443.2\end{array}$

$\begin{array}{lll}78 & 51 & 51136.8\end{array}$

$\begin{array}{lll}78 & 52 & \text { ?50697.6 }\end{array}$

$\begin{array}{lll}78 & 53 & ? 50491.8\end{array}$

$\begin{array}{lll}78 & 54 & ? 50681\end{array}$

$\begin{array}{lll}78 & 55 & ? 51161\end{array}$

$\begin{array}{lll}78 & 56 & ? 52037\end{array}$

$\begin{array}{lll}78 & 57 & ? 53050.2\end{array}$

$\begin{array}{lll}78 & 58 & ? 53641.2\end{array}$

$\begin{array}{lll}78 & 59 & ? 53276.6\end{array}$

$\begin{array}{lll}78 & 60 & 52723.2\end{array}$

$\begin{array}{lll}78 & 61 & 52285.6\end{array}$

$\begin{array}{lll}78 & 62 & 52064.8\end{array}$

$\begin{array}{lll}78 & 63 & 51948.8\end{array}$

$\begin{array}{lll}78 & 64 & 51874.6\end{array}$

$\begin{array}{lll}78 & 65 & 51825.0\end{array}$

$\begin{array}{lll}78 & 66 & 51791.8\end{array}$

$\begin{array}{lll}78 & 67 & 51770.2\end{array}$

$\begin{array}{lll}78 & 68 & 51746.8\end{array}$

$\begin{array}{lll}78 & 69 & 51716.4\end{array}$

$\begin{array}{lll}78 & 70 & 51682.0\end{array}$

$\begin{array}{lll}78 & 71 & 51630.8\end{array}$

$\begin{array}{lll}78 & 72 & 51563.4\end{array}$

$\begin{array}{lll}78 & 73 & 51536.4\end{array}$

$\begin{array}{lll}78 & 74 & 51563.8\end{array}$

$\begin{array}{lll}78 & 75 & 51609.4\end{array}$

$\begin{array}{lll}78 & 76 & 51659.2\end{array}$

$\begin{array}{lll}78 & 77 & 51685.0\end{array}$

$\begin{array}{lll}78 & 78 & 51706.6\end{array}$

$8178 \quad 51708.6$

$8177 \quad 51667.0$

$8176 \quad 51600.2$

$8175 ? 51491$

$\begin{array}{lll}81 & 74 & ? 51797\end{array}$

$\begin{array}{lll}81 & 73 & ? 50113\end{array}$

$\begin{array}{lll}81 & 72 & ? 51166.6\end{array}$

$8171 \quad 51531.4$

$8170 \quad 51656.6$

$\begin{array}{lll}81 & 69 & 51712.8\end{array}$

$\begin{array}{lll}81 & 68 & 51749.4\end{array}$

$\begin{array}{lll}81 & 67 & 51777.4\end{array}$

$81 \quad 66 \quad 51803.6$

$\begin{array}{lll}81 & 65 & 51836.8\end{array}$

$\begin{array}{lll}81 & 64 & 51884.2\end{array}$

$\begin{array}{lll}81 & 63 & 51957.8\end{array}$

$\begin{array}{lll}81 & 62 & 52084.6\end{array}$

$\begin{array}{lll}81 & 61 & 52305.8\end{array}$

$\begin{array}{lll}81 & 60 & 52679.4\end{array}$

$\begin{array}{lll}81 & 59 & ? 53193.2\end{array}$
51435.37 .9

51128.97 .9

50689.87 .8

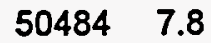

50673.37 .7

51153.37 .7

52029.37 .7

53042.67 .6

53633.77 .5

53269.17 .5

52715.87 .4

52278.27 .4

52057.57 .3

51941.57 .3

51867.37 .3

51817.87 .2

51784.67 .2

51763.17 .1

51739.77 .1

51709.47 .0

$51675 \quad 7.0$

51623.87 .0

51556.56 .9

51529.56 .9

$51557 \quad 6.8$

$51602.6 \quad 6.8$

$\begin{array}{lll}51652.5 & 6.7\end{array}$

51678.36 .7

$51700 \quad 6.6$

$51703.3 \quad 5.3$

51661.75 .3

$51595 \quad 5.2$

$51485.8 \quad 5.2$

51791.95 .1

$50108 \quad 5.0$

$51161.6 \quad 5.0$

$51526.5 \quad 4.9$

$51651.7 \quad 4.9$

$51708 \quad 4.8$
51744.6

$51744.6 \quad 4.8$

$51772.6 \quad 4.8$

51798.94 .7

51832.14 .7

$51879.6 \quad 4.6$

51953.24 .6

52080.14 .5

$52301.3 \quad 4.5$

52674.94 .5

$53188.8 \quad 4.4$
BOTTOM FIELD (nT)

GRAD. (Uncorr.) (Corr.) (Diff.) (nT/m) $\begin{array}{llll}51380.6 & 51370.1 & 10.5 & -102.6\end{array}$

$\begin{array}{llll}\mathbf{3} 51171.6 & 51161.1 & 10.5 & 57.1\end{array}$

$\begin{array}{llll}? 50220 & 50209.6 & 10.4 & -783.0\end{array}$

$\begin{array}{llll}249688 & 49677.6 & 10.4 & -1317.7\end{array}$

$\begin{array}{llll}? 49998.2 & 49987.8 & 10.4 & -1119.4\end{array}$

$\begin{array}{llll}350505.2 & 50494.8 & 10.4 & -1075.1\end{array}$

$\begin{array}{llll}251952.2 & 51941.9 & 10.3 & -139.0\end{array}$

$\begin{array}{llll}\mathbf{3} 53566.2 & 53555.9 & 10.3 & 845.9\end{array}$

$\begin{array}{llll}? 55051 & 55040.7 & 10.3 & 2311.1\end{array}$

$\begin{array}{lllll}? 53792 & 53781.8 & 10.2 & 844.9\end{array}$

$\begin{array}{llll}352682.6 & 52672.4 & 10.2 & -66.6\end{array}$

$\begin{array}{llll}52153.4 & 52143.2 & 10.2 & -216.7\end{array}$

$\begin{array}{llll}51968.2 & 51958.0 & 10.2 & -158.4\end{array}$

$\begin{array}{llll}51874.4 & 51864.3 & 10.1 & -122.0\end{array}$

$\begin{array}{llll}51814.0 & 51803.9 & 10.1 & -99.3\end{array}$

$\begin{array}{llll}51778.4 & 51768.3 & 10.1 & -76.4\end{array}$

$\begin{array}{llll}51755.6 & 51745.5 & 10.1 & -59.3\end{array}$

$\begin{array}{llll}51741.6 & 51731.6 & 10.0 & -46.9\end{array}$

$\begin{array}{llll}51723.8 & 51713.8 & 10.0 & -37.7\end{array}$

$\begin{array}{llll}51703.2 & 51693.2 & 10.0 & -21.6\end{array}$

$\begin{array}{llll}51675.0 & 51665.0 & 10.0 & -11.5\end{array}$

$\begin{array}{llll}51626.6 & 51616.6 & 10.0 & -6.9\end{array}$

$\begin{array}{llll}51551.8 & 51541.9 & 9.9 & -19.0\end{array}$

$\begin{array}{llll}51488.2 & 51478.3 & 9.9 & -79.0\end{array}$

$\begin{array}{llll}51523.0 & 51513.1 & 9.9 & -66.9\end{array}$

$\begin{array}{llll}51585.0 & 51575.1 & 9.9 & -40.0\end{array}$

$\begin{array}{llll}51631.4 & 51621.6 & 9.8 & -45.6\end{array}$

$\begin{array}{llll}51660.6 & 51650.8 & 9.8 & -40.0\end{array}$

$\begin{array}{llll}51707.0 & 51697.9 & 9.1 & -2.6\end{array}$

$\begin{array}{llll}51509.0 & 51500.0 & 9.0 & -149.5\end{array}$

$\begin{array}{llll}\mathbf{2} 51133.4 & 51124.4 & 9.0 & -586.2\end{array}$

$\begin{array}{llll}250186.2 & 50177.2 & 9.0 & -2640.7\end{array}$

$\begin{array}{llll}352175.4 & 52166.5 & 8.9 & 3381.0\end{array}$

$\begin{array}{llll}? 51193.4 & 51184.5 & 8.9 & 43.9\end{array}$

$\begin{array}{llll}51544.6 & 51535.7 & 8.9 & 21.6\end{array}$

$\begin{array}{llll}51661.8 & 51652.9 & 8.9 & 8.5\end{array}$

$\begin{array}{llll}51711.8 & 51703.0 & 8.8 & -1.6\end{array}$

$\begin{array}{llll}51740.6 & 51731.8 & 8.8 & -14.4\end{array}$

$\begin{array}{llll}51759.6 & 51750.8 & 8.8 & -29.2\end{array}$

$\begin{array}{llll}51777.4 & 51768.6 & 8.8 & -43.0\end{array}$

$\begin{array}{lllll}51798.4 & 51789.7 & 8.7 & -62.9\end{array}$

$\begin{array}{llll}51830.2 & 51821.5 & 8.7 & -88.5\end{array}$

$\begin{array}{llll}51879.8 & 51871.1 & 8.7 & -127.9\end{array}$

$\begin{array}{llll}51978.2 & 51969.5 & 8.7 & -174.4\end{array}$

$\begin{array}{lllll}\mathbf{2 5 2 1 8 7 . 8} & 52179.2 & 8.6 & -193.4\end{array}$

$\begin{array}{lllll}? 53696.4 & 53687.8 & 8.6 & 824.9\end{array}$ $\begin{array}{llll}51686.2 & 51676.4 & 9.8 & -33.4\end{array}$

$\begin{array}{llll}51635.6 & 51626.5 & 9.1 & -51.5\end{array}$

$\begin{array}{lllll}? 52652.2 & 52643.6 & 8.6 & -44.6\end{array}$ 
TOP FIELD (nT)

\begin{tabular}{|c|c|c|c|c|c|c|c|c|c|c|c|c|}
\hline INI & $A Y$ & & $\mathbf{s}$ & $\begin{array}{l}x \\
81\end{array}$ & & & $\begin{array}{l}\text { irr.) } \\
24.8\end{array}$ & $\begin{array}{l}\text { (Diff.) } \\
4.4\end{array}$ & $\begin{array}{l}\text { (Uncorr.) } \\
? 54526.4\end{array}$ & $\begin{array}{c}\text { (Corr.) } \\
54517.8\end{array}$ & $\begin{array}{c}\text { (Diff.) } \\
8.6\end{array}$ & $\begin{array}{l}\text { (nT/m) } \\
1798.7\end{array}$ \\
\hline $\begin{array}{l}28 \\
28\end{array}$ & $\begin{array}{l}196 \\
196\end{array}$ & 101922 & $\begin{array}{l}518 \\
520\end{array}$ & $\begin{array}{l}81 \\
81\end{array}$ & 57 & $? 52877.4$ & 52873.1 & $\begin{array}{l}4.4 \\
4.3\end{array}$ & ?53744.4 & 53735.9 & 8.5 & 1421.3 \\
\hline 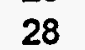 & 196 & 101935 & 522 & 81 & 6 & $? 51951.2$ & 51946.9 & 4.3 & $? 51706$ & 51697.5 & 8.5 & -402.0 \\
\hline 28 & 196 & 101947 & 524 & 81 & 5 & $? 51279$ & 51274.8 & 4.2 & ?50723.6 & 50715.1 & 8.5 & -910.5 \\
\hline 28 & 196 & 102000 & 526 & 81 & $A$ & ?51089 & 51084.8 & 4.2 & 50952.4 & 50943.9 & .5 & -223.9 \\
\hline 28 & 196 & 102013 & 528 & 81 & 3 & 50896.2 & 0892.1 & 4.1 & $? 50638.2$ & 50629.7 & 8.5 & -423.0 \\
\hline 28 & 196 & 102026 & 530 & 81 & 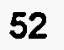 & $? 50710.8$ & 50706.7 & 4.1 & $? 54668.8$ & 4660.4 & 8.4 & 6488.5 \\
\hline 28 & 196 & 102039 & 532 & 81 & 1 & $? 51040.4$ & 51036.4 & 4.0 & $? 50640$ & 50631.6 & 8.4 & -656.4 \\
\hline 28 & 196 & 102051 & 534 & 81 & 0 & 49.8 & 51545.8 & 4.0 & 51543.0 & 51534.6 & & -11.1 \\
\hline 28 & 196 & 102104 & 536 & 81 & $y$ & 51958.0 & 1954.1 & 3.9 & 52106.0 & 52097.6 & 8.4 & 242.6 \\
\hline 28 & 196 & 102116 & 538 & 81 & 8 & $? 52317.4$ & 52313.5 & 3.9 & $? 52666.4$ & 52658.1 & 8.3 & 572.1 \\
\hline 28 & 196 & 102129 & 540 & 81 & 7 & $? 52472$ & 52468.1 & 3.9 & $? 53079$ & 53070.7 & .3 & 95.1 \\
\hline 28 & 196 & 102141 & 542 & 81 & 6 & ?52315.4 & 52311.6 & 3.8 & $? 52662.8$ & 52654.5 & .3 & 569.5 \\
\hline 28 & 196 & 102154 & 544 & 81 & 5 & 51921.8 & 51918 & 3.8 & ?51834.2 & 51825.9 & 8.3 & -143.6 \\
\hline 28 & 196 & 102205 & 546 & 81 & 4 & 51733.8 & 51730.1 & 3.7 & 51612.6 & 51604.4 & 8.2 & -198.7 \\
\hline 28 & 196 & 102216 & 548 & 81 & 3 & 51731.0 & 51727.3 & 3.7 & 8.2 & 51600.0 & 8.2 & -201.3 \\
\hline 28 & 196 & 102228 & 550 & 81 & 2 & 51861.6 & 51858 & 3.6 & 1.8 & 51813.6 & 8.2 & -65.2 \\
\hline 28 & 196 & 102240 & 552 & 81 & 1 & 2062.0 & 52058.4 & 3.6 & 3.6 & 5.4 & 8.2 & 199.3 \\
\hline 28 & 196 & 102253 & 554 & 81 & 0 & ?52232.4 & 52228.8 & 3.6 & 4.4 & 6.3 & .1 & 462.3 \\
\hline 28 & 196 & 102305 & 556 & 81 & 9 & ?52299.2 & 52295.7 & 3.5 & 92 & 3.9 & .1 & 480.0 \\
\hline 28 & 196 & 102318 & 558 & 81 & 8 & $? 52162.6$ & 52159.1 & 3.5 & 0.8 & 02.7 & 8.1 & 406.9 \\
\hline 2 & 196 & 102330 & 560 & 81 & 7 & 51867.4 & 51864 & 3.4 & 6.2 & 8.1 & 8. & -18.4 \\
\hline 28 & 196 & 102342 & 562 & 81 & (6) & 51652.6 & 51649.2 & 3.4 & 32.4 & 4.3 & 8.1 & -279.0 \\
\hline 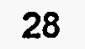 & 196 & 102353 & 564 & 81 & 5 & 51648.2 & 51644.9 & 3.3 & 5.8 & 7.8 & 8.0 & -233.4 \\
\hline 28 & 196 & 102407 & 566 & 81 & 4 & 518 & 51830.7 & 3.3 & 52.6 & 51744.6 & 8.0 & -133.4 \\
\hline 28 & 196 & 102425 & 568 & 81 & 3 & 52016.6 & 52013.4 & 3.2 & & 32.0 & 8.0 & -207.5 \\
\hline 28 & 196 & 102438 & 570 & 81 & 2 & 52255.8 & 52252.6 & 3.2 & 40.8 & 32.9 & 7.9 & -188.5 \\
\hline 28 & 196 & 102451 & 572 & 81 & 31 & $? 52602.2$ & 52599.1 & 3.1 & 42.6 & 52534.7 & 7.9 & -97.7 \\
\hline 28 & 196 & 102504 & 574 & 81 & 0 & ?53084 & 53080.9 & 3.1 & 8.4 & 0.5 & 7.9 & -173.1 \\
\hline 28 & 196 & 102517 & 576 & 81 & 29 & ?53392.8 & 53389.8 & 3.0 & 78.6 & 0.7 & 7.9 & -23.3 \\
\hline 28 & 196 & 102529 & 578 & 81 & 28 & ?52906.8 & 52903.8 & 3.0 & 37 & 9.2 & 7.8 & 9229.8 \\
\hline 2 & 196 & 102542 & 580 & 81 & 7 & ?50902.8 & 50899.9 & 2.9 & 7.6 & 9.8 & 7.8 & 1958.7 \\
\hline 28 & 196 & 102555 & 582 & 81 & 26 & ?52133.4 & 52130.5 & 2.9 & 0.2 & 2.4 & 7.8 & 10535.7 \\
\hline 28 & 196 & 102609 & 584 & 81 & 25 & ?52129.2 & 52126.3 & 2.9 & 9.8 & 2.0 & 7.8 & 50.2 \\
\hline 28 & 196 & 102622 & 586 & 81 & 24 & $? 52277.6$ & 52274.8 & 2.8 & ?16274.2 & 16266.5 & 7.7 & -59022.0 \\
\hline 28 & 196 & 102635 & 588 & 81 & 23 & $? 48509.6$ & 48506.8 & 2.8 & ?52109.6 & 52101.9 & 7.7 & 5901.6 \\
\hline 28 & 196 & 102650 & 590 & 81 & 22 & $? 50430.4$ & 50427.7 & 2.7 & $? 50959.4$ & 50951.7 & 7.7 & 867.2 \\
\hline 28 & 196 & 102703 & 592 & 81 & 21 & 58.2 & 51255.5 & 2.7 & 574 & 51566.3 & 7.7 & 517.7 \\
\hline 28 & 196 & 102715 & 594 & 81 & 20 & 51533.8 & 51531.2 & 2.6 & $? 51$ & 35.8 & 7.6 & 343.6 \\
\hline 28 & 196 & 102727 & 596 & 81 & 19 & 51640.6 & 51638 & 2.6 & 5.8 & 8.2 & 7.6 & 205.2 \\
\hline $2 \varepsilon$ & 196 & 102738 & 598 & 81 & 18 & 51686.0 & 51683.5 & 2.5 & 3.4 & 5.8 & 7.6 & 126.9 \\
\hline 28 & 196 & 102749 & 600 & 81 & 17 & 51702.0 & 51699.5 & 2.5 & 51750.8 & 51743.2 & 7.6 & 0.0 \\
\hline 28 & 196 & 102801 & 602 & 81 & 16 & 51710.4 & 51708 & 2.4 & 51747.2 & 51739.6 & 7.6 & 60.3 \\
\hline 28 & 196 & 102812 & 604 & 81 & 15 & 51713.2 & 51710.8 & 2.4 & 51742.0 & 51734.5 & 7.5 & 47.2 \\
\hline 28 & 196 & 102824 & 606 & 81 & 14 & 51714.0 & 51711.6 & 2.4 & 2.8 & 51725.3 & 7.5 & 30.8 \\
\hline $2 \varepsilon$ & 196 & 102837 & 608 & 81 & 13 & 51713.8 & 51711.5 & 2.3 & 51734.0 & 26.5 & 7.5 & 33.1 \\
\hline 28 & 196 & 102850 & 610 & 81 & 12 & 51711.6 & 51709.3 & 2.3 & 51724.6 & 51717.1 & 7.5 & 21.3 \\
\hline 28 & 196 & 102901 & 612 & 81 & 11 & 51708.6 & 51706.4 & 2.2 & 51719.2 & 51711.8 & 7.4 & 17.4 \\
\hline & 196 & 102912 & 614 & 81 & 10 & 51707.2 & 51705 & 2.2 & 51715.6 & 51708.2 & 7.4 & 13.8 \\
\hline
\end{tabular}

BOTTOM FIELD (nT) GRAD.

(Uncorr.) (Corr.) (Diff.) (nT/m)

$\begin{array}{llll}751706 & 51697.5 & 8.5 & -402.0\end{array}$

$\begin{array}{lllll}50952.4 & 50943.9 & 8.5 & -223.9\end{array}$

$\begin{array}{lllll}254668.8 & 54660.4 & 8.4 & 6488.5\end{array}$

$\begin{array}{llll}? 50640 & 50631.6 & 8.4 & -656.4\end{array}$

$\begin{array}{lllll}51543.0 & 51534.6 & 8.4 & -11.1\end{array}$

$\begin{array}{lllll}52106.0 & 52097.6 & 8.4 & 242.6\end{array}$

$\begin{array}{llll}52666.4 & 52658.1 & 8.3 & 572.1\end{array}$

$\begin{array}{llll}252662.8 & 52654.5 & 8.3 & 569.5\end{array}$

$\begin{array}{lllll}251834.2 & 51825.9 & 8.3 & -143.6\end{array}$

$51608.2 \quad 51600.0 \quad 8.2 \quad-201.3$

$\begin{array}{llll}51821.8 & 51813.6 & 8.2 & -65.2\end{array}$

$\begin{array}{lllll}252514.4 & 52506.3 & 8.1 & 462.3\end{array}$

\begin{tabular}{l}
$252592 \quad 52583.9$ \\
\hline
\end{tabular}

$\begin{array}{lllll}251856.2 & 51848.1 & 8.1 & -18.4\end{array}$

$\begin{array}{lllll}251482.4 & 51474.3 & 8.1 & -279.0\end{array}$

$\begin{array}{lllll}251752.6 & 51744.6 & 8.0 & -133.4\end{array}$

$\begin{array}{lllll}? 51890 & 51882.0 & 8.0 & -207.5\end{array}$

$\begin{array}{lllll}252140.8 & 52132.9 & 7.9 & -188.5\end{array}$

$\begin{array}{lllll}252542.6 & 52534.7 & 7.9 & -97.7\end{array}$

$\begin{array}{lllll}552978.4 & 52970.5 & 7.9 & -173.1\end{array}$

$\begin{array}{llll}252097.6 & 52089.8 & 7.8 & 1958.7\end{array}$

$\begin{array}{lllll}258560.2 & 58552.4 & 7.8 & 10535.7\end{array}$

$\begin{array}{lllll}? 52159.8 & 52152.0 & 7.8 & 50.2\end{array}$

$\begin{array}{llll}216274.2 & 16266.5 & 7.7 & -59022.0\end{array}$

$\begin{array}{lll}52109.6 & 52101.9 & 7.7\end{array}$

$\begin{array}{lll}50959.4 & 50951.7 & 7.7\end{array}$

$\begin{array}{llll}51743.4 & 51735.8 & 7.6 & 343.6\end{array}$

$\begin{array}{llll}51765.8 & 51758.2 & 7.6 & 205.2\end{array}$

126.9
?50723.6 $50715.1 \quad 8.5 \quad-910.5$

$\begin{array}{lllll}52183.6 & 52175.4 & 8.2 & 199.3\end{array}$

$\begin{array}{lllll}\text { ?53378.6 } & 53370.7 & 7.9 & -23.3\end{array}$ 
TOP FIELD (nT)

\begin{tabular}{cccc} 
LINE & DAY & TIME & STA. \\
\hline 28 & 196 & 102923 & 616 \\
28 & 196 & 102934 & 618 \\
28 & 196 & 102946 & 620 \\
28 & 196 & 102958 & 622 \\
28 & 196 & 103010 & 624 \\
28 & 196 & 103021 & 626 \\
28 & 196 & 103032 & 628 \\
28 & 196 & 103043 & 630 \\
28 & 196 & 103055 & 632 \\
28 & 196 & 103107 & 634 \\
29 & 196 & 122911 & 638 \\
29 & 196 & 122924 & 640 \\
29 & 196 & 122937 & 642 \\
29 & 196 & 122949 & 644 \\
29 & 196 & 123001 & 646 \\
29 & 196 & 123015 & 648 \\
29 & 196 & 123035 & 650 \\
29 & 196 & 123046 & 652 \\
29 & 196 & 123058 & 654 \\
29 & 196 & 123109 & 656 \\
29 & 196 & 123122 & 658 \\
29 & 196 & 123134 & 660 \\
29 & 196 & 123145 & 662 \\
29 & 196 & 123157 & 664 \\
29 & 196 & 123214 & 666 \\
29 & 196 & 123226 & 668 \\
29 & 196 & 123237 & 670 \\
29 & 196 & 123249 & 672 \\
29 & 196 & 123301 & 674 \\
29 & 196 & 123314 & 676 \\
29 & 196 & 123326 & 678 \\
29 & 196 & 123337 & 680 \\
29 & 196 & 123349 & 682 \\
29 & 196 & 123400 & 684 \\
29 & 196 & 123411 & 686 \\
29 & 196 & 123424 & 688 \\
29 & 196 & 123437 & 690 \\
29 & 196 & 123450 & 692 \\
29 & 196 & 123504 & 694 \\
29 & 196 & 123518 & 696 \\
29 & 196 & 123532 & 698 \\
29 & 196 & 123548 & 700 \\
29 & 196 & 123601 & 702 \\
29 & 196 & 123614 & 704 \\
29 & 196 & 123625 & 706 \\
29 & 196 & 123637 & 708 \\
29 & 196 & 123654 & 710 \\
29 & 196 & 123706 & 712 \\
29 & 196 & 123721 & 714
\end{tabular}

$\begin{array}{lll}81 & 4 & 51677.2 \\ 81 & 3 & 51660.0\end{array}$

$\begin{array}{lll}81 & 2 & 51640.0\end{array}$

$\begin{array}{lll}81 & 1 & 51609.2\end{array}$

$\begin{array}{lll}81 & 0 & 51561.8\end{array}$

$\begin{array}{lll}84 & 0 & 51513.8\end{array}$

84151594.2

$\begin{array}{lll}84 & 2 & 51624.2\end{array}$

$\begin{array}{lll}84 & 3 & 51629.6\end{array}$

$\begin{array}{lll}84 & 4 & 51644.6\end{array}$

$\begin{array}{lll}84 & 5 & 51739.6\end{array}$

$\begin{array}{lll}84 & 6 & 51721.8\end{array}$

$\begin{array}{lll}84 & 7 & 51693.0\end{array}$

$\begin{array}{lll}84 & 8 & 51686.8\end{array}$

$\begin{array}{lll}84 & 9 & 51686.4\end{array}$

$\begin{array}{lll}84 & 10 & 51687.2\end{array}$

$\begin{array}{lll}84 & 11 & 51687.8\end{array}$

$\begin{array}{lll}84 & 12 & 51686.6\end{array}$

8413

8414

8415

8416

8417

8418

8419

8420

8421

8422

8423

8424

8425

8426

$\begin{array}{ll}84 & 27\end{array}$

8428

8429

$\begin{array}{lll}84 & 30 & ? 55047\end{array}$

$\begin{array}{lll}84 & 31 & ? 53703.2\end{array}$

$\begin{array}{lll}84 & 32 & 52784.8\end{array}$

$\begin{array}{lll}84 & 33 & 52383.8\end{array}$

$\begin{array}{lll}84 & 34 & 52145.0\end{array}$

$\begin{array}{lll}84 & 35 & 51703.2\end{array}$

$\begin{array}{lll}84 & 36 & ? 51448.4\end{array}$

$\begin{array}{lll}84 & 37 & 51486.4\end{array}$

$\begin{array}{lll}84 & 38 & 51597.2\end{array}$
BOTTOM FIELD (nT)

$\begin{array}{llllll}\mathbf{X} & \mathbf{Y} & \text { (Uncorr.) } & \text { (Corr.) } & \text { (Diff.) } & \text { (Un } \\ 81 & 9 & 51705.8 & \mathbf{5 1 7 0 3 . 6} & 2.2 & 517 \\ 81 & 8 & 51702.6 & 51700.5 & 2.1 & 517 \\ 81 & 7 & 51698.8 & 51696.7 & 2.1 & 517 \\ 81 & 6 & 51697.2 & 51695.2 & 2.0 & 516 \\ 81 & 5 & 51694.4 & 51692.4 & 2.0 & 5 \\ 81 & 4 & 51677.2 & 51675.3 & 1.9 & 516\end{array}$

$51658.1 \quad 1.9$

$51638.1 \quad 1.9$

$51607.4 \quad 1.8$

$51560 \quad 1.8$

$51523.8-10.0$

$51604.3-10.1$

$51634.3-10.1$

$51639.7-10.1$

$51654.8-10.2$

$51749.8-10.2$

$\begin{array}{lll}51732 & -10.2\end{array}$

$51703.2-10.2$

$51697.1-10.3$

$51696.7-10.3$

$51697.5-10.3$

$51698.1-10.3$

$51696.9-10.3$

$51695.4-10.4$

$51692-10.4$

$51685.8-10.4$

$51675.4-10.4$

$51659.4-10.4$

$51632.9-10.5$

$51584.7-10.5$

$51503.7-10.5$

$51195.7-10.5$

$50981.6-10.6$

$50877.6-10.6$

$51091.6-10.6$

$52083.6-10.6$

$55493-10.6$

$58606.1-10.7$

$58578.1-10.7$

$55057.7-10.7$

$53713.9-10.7$

$52795.6-10.8$

$52394.6-10.8$

$52155.8-10.8$

$51714-10.8$

$51459.2-10.8$

$51497.3-10.9$

$51608.1-10.9$
$51380.7-10.5$

\section{(Uncorr) (Corr)}

51706.2

51711.8

51701.0

51698.4

51691.6

51667.0

51649.6

51615.0

51540.0

?52195.2

51411.0

51546.0

51598.4

51602.2

51558.2

?51935.6

51743.0

51681.4

51679.6

51681.8

51684.8

51685.8

51687.2

51687.2

51686.0

51684.0

51677.8

51667.2

51647.8

51610.8

51537.6

51416.6

51219.4

50930.8

?50678.8

?50654

?51802.6

?58581.4

?58600.6

?58600.6

?52068.4

?53862.2

$? 52717$

$? 52292.2$

?52387.8

?51505.6

$? 51160$

51308.6

51455.4
(Uncorr.)
51713.6

51704.4

51693.6

51691.1

51684.3

51659.7

51642.3

51607.8

51532.8

52188.0

51418.9

51554.0

51606.4

51610.2

51566.3

51943.7

51751.1

51689.6

51687.8

51690.0

51693.0

51694.1

51695.5

51695.5

51694.4

51692.4

51686.2

51675.6

51656.3

51619.3

51546.1

51425.1

51228.0

50939.4

50687.4

50662.6

51811.3

58590.1

58609.3

58609.3

52077.2

53871.0

52725.8

52301.1

52396.7

51514.5

51169.0

51317.6

51464.4
GRAD.

Diff.) (nT/m)

$7.4 \quad 12.8$

$7.4 \quad 15.1$

$7.4 \quad 3.6$

$7.3 \quad 2.0$

$7.3-4.6$

$7.3-16.7$

$7.3-17.0$

$7.2-41.0$

$7.2 \quad-113.4$

$7.2 \quad 1038.4$

$-7.9 \quad-168.5$

$-8.0 \quad-79.0$

$-8.0 \quad-42.3$

$-8.0 \quad-44.9$

$-8.1 \quad-141.6$

$-8.1321 .3$

$-8.1 \quad 34.8$

$-8.2 \quad-19.0$

$-8.2 \quad-11.8$

$-8.2 \quad-7.5$

$-8.2 \quad-3.9$

$-8.3-3.3$

$-8.3 \quad 1.0$

$-8.3 \quad 3.6$

$-8.4 \quad 7.2$

$-8.4 \quad 14.1$

$-8.4 \quad 21.0$

$-8.4 \quad 29.8$

$-8.5 \quad 41.6$

$-8.5 \quad 60.0$

$-8.5 \quad 72.8$

$-8.5 \quad 76.1$

$-8.6 \quad 56.1$

$-8.6$

$-65.9$

$-8.6 \quad-308.5$

$-8.6 \quad-700.0$

$-8.7 \quad-443.3$

$-8.7 \quad 5080.3$

$\begin{array}{ll}-8.7 & 8.5\end{array}$

$-8.7 \quad 54.4$

$-8.8$

$-8.8$

$-4883.0$

260.7

$-8.8-111.1$

$-8.9 \quad-150.2$

$-8.9 \quad 398.0$

$-8.9-323.9$

$-9.0 \quad-472.8$

$-9.0 \quad-291.5$

$-9.0 \quad-232.5$ 
TOP FIELD (nT)

\begin{tabular}{|c|c|c|c|c|c|c|c|c|c|c|c|c|}
\hline & & & & & & & & & & 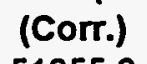 & $100^{\circ}$ & 0 \\
\hline 29 & & & & & & & & -10.9 & & & -9.0 & \\
\hline 29 & 196 & 3374 & 718 & 84 & 40 & & 6.5 & -10.9 & & 1789.7 & 01 & \\
\hline 29 & 196 & 3758 & 720 & 84 & 41 & 55.0 & & & & & & \\
\hline 29 & 196 & 23809 & 722 & 84 & 42 & 32.4 & 12 & & & 90.3 & 9.1 & 3.9 \\
\hline 29 & 96 & 23820 & 724 & 84 & 43 & 97.8 & 08.8 & & & & -9.1 & -362.9 \\
\hline 29 & 96 & & 726 & 84 & 44 & & & & & & -9.2 & 09.2 \\
\hline 29 & & & 728 & 84 & 45 & & 49.6 & & & & -9.2 & 9.8 \\
\hline$x$ & 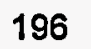 & 59 & 730 & 84 & 46 & & & -11.1 & & & -9.2 & 100.3 \\
\hline 29 & 96 & 11 & 732 & 84 & 47 & 0.6 & 31.7 & -11.1 & & & -9.2 & 27.5 \\
\hline 25 & 96 & 123924 & 734 & 84 & 48 & & 0.1 & -11.1 & & & -9.3 & 5.6 \\
\hline 29 & 196 & 123936 & 736 & 84 & 49 & 0.2 & 1.3 & & & & -9.3 & 0.3 \\
\hline $2 \varsigma$ & 196 & 23949 & 738 & 84 & 50 & 32.2 & 3.3 & & & & -9.3 & 4.3 \\
\hline 25 & 196 & 01 & 740 & 84 & 51 & & 9.2 & & & & -9.4 & -623.3 \\
\hline 29 & 196 & 13 & 742 & 84 & 52 & & 20.4 & & & 8.4 & -9.4 & -1770.8 \\
\hline & 96 & 0 & 744 & 84 & 53 & & & & & & -9.4 & 78.4 \\
\hline 29 & 96 & 10 & 746 & 84 & 54 & $? 5$ & 3.8 & -1 & & & -9.4 & \\
\hline & & 3 & 748 & 84 & 55 & & & -1 & & & -9.0 & \\
\hline & 96 & 66 & 750 & 84 & 56 & 3.2 & & & & & -9.5 & \\
\hline 29 & 96 & 124 & 752 & 84 & 57 & & 2.1 & & & & 9.5 & \\
\hline & 96 & 30 & 754 & 84 & 58 & 55.4 & 6.7 & & & & 9.5 & \\
\hline & 96 & 4 & 756 & 84 & 59 & 18.2 & 9.5 & & & & -9.6 & \\
\hline & 96 & 7 & 758 & 84 & 60 & 2 & 3.5 & & & & -9.6 & \\
\hline & 96 & 24210 & 760 & 84 & 61 & & & & & & -9.6 & \\
\hline & 96 & 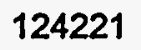 & 762 & 84 & 62 & & & & & & 0 & \\
\hline & 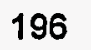 & & 764 & 84 & 63 & & & & & & -9 & \\
\hline 0 & & & 766 & 84 & 64 & & & & & & -9 & \\
\hline & 96 & & $76 \varepsilon$ & 84 & 65 & & & & & 3.9 & -9 & \\
\hline & & & 770 & 84 & 66 & & 0.1 & & & & -9 & \\
\hline & 9 & & 772 & 84 & 67 & & .7 & & & & -9 & \\
\hline & & & 774 & 84 & 68 & & & & & & -9 & \\
\hline & 06 & 7 & 776 & 84 & 69 & & & & & & -9 & \\
\hline & 06 & & 778 & 84 & 70 & & & & & & -99 & \\
\hline 29 & 0 & 124414 & 780 & 84 & 71 & & & & & & 0 & \\
\hline 29 & 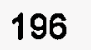 & & 782 & 84 & 72 & & & & & & -9.9 & \\
\hline 29 & 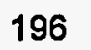 & & 784 & 84 & 73 & & & & & & -9.9 & \\
\hline 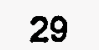 & 9 & & 78 & 84 & 74 & & & & & & -1 & \\
\hline & & & 10 & 84 & 75 & & & & & & .0 & \\
\hline & & & 79 & 84 & 76 & & & & & & 0.0 & \\
\hline & & & 79 & 84 & 77 & & & & & & & \\
\hline & 0 & & 794 & 84 & 78 & & & & & & & \\
\hline 30 & 196 & & 796 & 87 & 72 & & & & & & & \\
\hline 30 & 196 & 125053 & 798 & 87 & 71 & & 44.4 & & & & & 10.5 \\
\hline 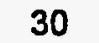 & 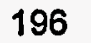 & 125 & 800 & 87 & 70 & 20.0 & 0.9 & & & & & \\
\hline 30 & 96 & & 80 & 87 & 69 & & & & & 4.6 & -10.8 & 4.4 \\
\hline & 9 & & 80 & 87 & 68 & & & & & & -10.8 & 1.5 \\
\hline & 1 & & 80 & 87 & 67 & & & & & & -10.9 & \\
\hline & & 125 & 80 & 87 & 66 & & & & & & & -59.7 \\
\hline & & 125211 & 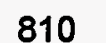 & 87 & 65 & & & & & & & -77.0 \\
\hline & & & & & & & & & & & & \\
\hline
\end{tabular}

BOTTOM FIELD (nT) GRAD. (nT/m) 
TOP FIELD (nT)

LINE DAY TIME STA $X \quad Y$ (UnCOTr)

$\begin{array}{llll}30 & 196 & 125234 & 814 \\ 30 & 196 & 125249 & 816 \\ 30 & 196 & 125305 & 818 \\ 30 & 196 & 125322 & 820 \\ 30 & 196 & 125334 & 822 \\ 30 & 196 & 125345 & 824 \\ 30 & 196 & 125357 & 826 \\ 30 & 196 & 125410 & 828 \\ 30 & 196 & 125423 & 830 \\ 30 & 196 & 125434 & 832 \\ 30 & 196 & 125448 & 834 \\ 30 & 196 & 125459 & 836 \\ 30 & 196 & 125511 & 838 \\ 30 & 196 & 125523 & 840 \\ 30 & 196 & 125535 & 842 \\ 30 & 196 & 125548 & 844 \\ 30 & 196 & 125600 & 846 \\ 30 & 196 & 125612 & 848\end{array}$

$\begin{array}{llll}30 & 196 & 125624 & 850\end{array}$

$\begin{array}{lllll}30 & 196 & 125635 & 852\end{array}$

$\begin{array}{lllll}30 & 196 & 125647 & 854\end{array}$

$\begin{array}{lllll}30 & 196 & 125701 & 856\end{array}$

$\begin{array}{lllll}30 & 196 & 125713 & 858\end{array}$

$\begin{array}{lllll}30 & 196 & 125725 & 860\end{array}$

$\begin{array}{lllll}30 & 196 & 125737 & 862\end{array}$

$\begin{array}{lllll}30 & 196 & 125749 & 864\end{array}$

$\begin{array}{lllll}30 & 196 & 125801 & 866\end{array}$

$\begin{array}{lllll}30 & 196 & 125812 & 868\end{array}$

$\begin{array}{llll}30 & 196 & 125823 & 870\end{array}$

$\begin{array}{llll}30 & 196 & 125835 & 872\end{array}$

$\begin{array}{lllll}30 & 196 & 125845 & 874\end{array}$

$\begin{array}{lllll}30 & 196 & 125856 & 876\end{array}$

$\begin{array}{llll}30 & 196 & 125907 & 878\end{array}$

$\begin{array}{llll}30 & 196 & 125918 & 880\end{array}$

$\begin{array}{llll}30 & 196 & 125930 & 882\end{array}$

$\begin{array}{llll}30 & 196 & 125949 & 884\end{array}$

$\begin{array}{llll}30 & 196 & 130000 & 886\end{array}$

$\begin{array}{llll}30 & 196 & 130010 & 888\end{array}$

$30 \quad 196 \quad 130021 \quad 890$

$\begin{array}{llll}30 & 196 & 130032 & 892\end{array}$

$\begin{array}{llll}30 & 196 & 130043 & 894\end{array}$

$\begin{array}{lllll}30 & 196 & 130054 & 896\end{array}$

$\begin{array}{llll}30 & 196 & 130105 & 898\end{array}$

$30 \quad 196 \quad 130116 \quad 900$

$\begin{array}{llll}30 & 196 & 130127 & 902\end{array}$

$30 \quad 196 \quad 130139 \quad 904$

$\begin{array}{llll}30 & 196 & 130150 & 906\end{array}$

$\begin{array}{llll}30 & 196 & 130201 & 908\end{array}$

$30 \quad 196 \quad 130212 \quad 910$

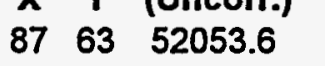

$\begin{array}{llllll}87 & 62 & 52270.0 & 52282.4 & -12.4\end{array}$

$\begin{array}{lll}87 & 61 & 52630.4\end{array}$

$\begin{array}{lll}87 & 60 & ? 53244.8\end{array}$

$\begin{array}{lll}87 & 59 & ? 53877.4\end{array}$

$\begin{array}{lll}87 & 58 & ? 53530.4\end{array}$

$\begin{array}{lll}87 & 57 & ? 52988.4\end{array}$

$\begin{array}{lll}87 & 56 & ? 51836\end{array}$

$\begin{array}{lll}87 & 55 & ? 51039\end{array}$

$\begin{array}{lll}87 & 54 & ? 51135.2\end{array}$

$\begin{array}{lll}87 & 53 & 51277.2\end{array}$

$\begin{array}{lll}87 & 52 & ? 51252.2\end{array}$

$\begin{array}{lll}87 & 51 & ? 51449.4\end{array}$

$\begin{array}{lll}87 & 50 & 51929.0\end{array}$

$\begin{array}{lll}87 & 49 & ? 52511\end{array}$

$\begin{array}{llll}87 & 48 & ? 52750.2\end{array}$

$\begin{array}{lll}87 & 47 & ? 52736.6\end{array}$

$\begin{array}{lll}87 & 46 & ? 52522\end{array}$

$\begin{array}{lll}87 & 45 & 51970.4\end{array}$

$\begin{array}{lll}87 & 44 & ? 51526.8\end{array}$

$\begin{array}{lll}87 & 43 & ? 51426.2\end{array}$

$\begin{array}{lll}87 & 42 & 51568.0\end{array}$

$\begin{array}{lll}87 & 41 & 51767.0\end{array}$

$\begin{array}{llll}87 & 40 & 51848.6\end{array}$

$\begin{array}{lll}87 & 39 & 51752.6\end{array}$

$\begin{array}{lll}87 & 38 & 51580.6\end{array}$

$\begin{array}{lll}87 & 37 & 51475.8\end{array}$

$\begin{array}{llll}87 & 36 & 51527.8\end{array}$

$\begin{array}{lll}87 & 35 & 51667.4\end{array}$

$\begin{array}{llll}87 & 34 & 51742.6\end{array}$

$\begin{array}{lll}87 & 33 & 51878.2\end{array}$

$\begin{array}{lll}87 & 32 & 52020.6\end{array}$

$\begin{array}{llll}87 & 31 & 52190.6\end{array}$

$\begin{array}{llll}87 & 30 & 52314.0\end{array}$

$\begin{array}{lll}87 & 29 & 52324.0\end{array}$

$\begin{array}{lll}87 & 28 & 52256.2\end{array}$

$\begin{array}{lll}87 & 27 & 52017.0\end{array}$

$\begin{array}{lll}87 & 26 & 51777.2\end{array}$

$\begin{array}{lll}87 & 25 & 51604.4\end{array}$

$\begin{array}{lll}87 & 24 & 51513.0\end{array}$

$\begin{array}{lll}87 & 23 & 51480.8\end{array}$

$\begin{array}{lll}87 & 22 & 51494.6\end{array}$

$\begin{array}{lll}87 & 21 & 51528.8\end{array}$

$\begin{array}{lll}87 & 20 & 51564.0\end{array}$

$\begin{array}{lll}87 & 19 & 51594.4\end{array}$

$\begin{array}{lll}87 & 18 & 51616.6\end{array}$

$\begin{array}{lll}87 & 17 & 51635.4\end{array}$

$\begin{array}{lll}87 & 16 & 51648.8\end{array}$

$\begin{array}{lll}87 & 15 & 51658.6\end{array}$

$53257.3-12.5$

$53889.9-12.5$

$53542.9-12.5$

$53000.9-12.5$

$51848.6-12.6$

$51051.6-12.6$

$51147.8-12.6$

$51289.8-12.6$

$51264.8-12.6$

$51462.1-12.7$

$\begin{array}{lll}51941.7 & -12.7\end{array}$

$52523.7-12.7$

$52762.9-12.7$

$52749.3-12.7$

$52534.8-12.8$

$51983.2-12.8$

$51539.6-12.8$

$\begin{array}{lll}51439 & -12.8\end{array}$

$51580.8-12.8$

$\begin{array}{lll}51779.9 & -12.9\end{array}$

$51861.5-12.9$

$51765.5-12.9$

$51593.5-12.9$

$51488.7-12.9$

$51540.8-13.0$

$51680.4-13.0$

$51755.6-13.0$

$51891.2-13.0$

$52033.6-13.0$

$52203.6-13.0$

$52327.1-13.1$

$52337.1-13.1$

$52269.3-13.1$

$52030.1-13.1$

$51790.3-13.1$

$51617.6-13.2$

$51526.2-13.2$

$51494-13.2$

$51507.8-13.2$

$\begin{array}{ll}51542 & -13.2\end{array}$

$51577.3-13.3$

$51607.7-13.3$

$51629.9-13.3$

$51648.7-13.3$

$51662.1-13.3$

$51671.9-13.3$
BOTTOM FIELD (nT)

GRAD.

(Corr.) (Diff.) (Uncorr.) (Corr.) (Diff.) (nT/m)

$\begin{array}{llllll}52066 & -12.4 & 51957.4 & 51968.4 & -11.0 & -157.7\end{array}$

$\begin{array}{llll}252197.2 & 52208.2 & -11.0 & -119.3\end{array}$

$\begin{array}{lllllll}52642.8 & -12.4 & ? 52523.6 & 52534.6 & -11.0 & -175.1\end{array}$
$? 53459$

$? 54952.2$

$? 55343.8$

$? 53322.8$

$? 51185.8$

$? 50112$

250834.8

$? 51417.8$

$? 50867$

$? 50999$

$? 51951$

$? 57009.6$

253356

?53137.6

?52895.8

$? 51766$

$? 51115.8$

?51024.4

51448.4

?51901.4

?52075.8

51866.6

51476.6

$? 51266$

51385.8

51637.4

?51619

51827.2

51963.0

52124.0

52238.8

$? 52265.4$

?52118.6

51874.6

51644.8

51507.8

51451.6

51452.6

51488.8

51533.2

51581.2

51612.8

51628.8

51646.2

51660.4

51668.8
$53470.1-11.1$

$54963.3-11.1$

$55354.9-11.1$

$53333.9-11.1$

$51197.0-11.2$

$50123.2-11.2$

$50846.0-11.2$

$51429.1-11.3$

$50878.3 \quad-11.3$

$51010.3-11.3$

$51962.3-11.3$

$57021.0 \quad-11.4$

$53367.4 \quad-11.4$

$53149.0-11.4$

$52907.2 \quad-11.4$

$51777.5-11.5$

$51127.3-11.5$

$51035.9-11.5$

$51459.9-11.5$

$51913.0-11.6$

$52087.4-11.6$

$51878.2-11.6$

$51488.2-11.6$

$51277.7-11.7$

$51397.5-11.7$

$51649.1-11.7$

$51630.7 \quad-11.7$

$51839.0-11.8$

$\begin{array}{lll}51974.8 & -11.8\end{array}$

$\begin{array}{lll}52135.8 & -11.8\end{array}$

$52250.6 \quad-11.8$

$\begin{array}{lll}52277.3 & -11.9\end{array}$

$52130.5-11.9$

$51886.5-11.9$

$51656.7-11.9$

$51519.8-12.0$

$51463.6-12.0$

$51464.6-12.0$

$51500.8-12.0$

$51545.3-12.1$

$\begin{array}{ll}51593.3 & -12.1\end{array}$

$51624.9-12.1$

$51640.9-12.1$

$51658.4-12.2$

$51672.6-12.2$

$51681.0-12.2$
351.1

1762.0

2972.8

548.2

$-1065.9$

$-1519.7$

$-492.5$

230.5

$-631.5$

$-738.4$

36.1

7374.8

993.1

657.4

612.8

$-335.1$

$-673.8$

$-658.7$

$-196.1$

220.3

372.5

186.9

$-170.5$

$-343.9$

$-232.8$

$-49.2$

$-202.6$

$-83.6$

$-94.4$

$-109.2$

$-123.3$

$-96.1$

$-225.6$

$-233.4$

$-217.0$

$-158.4$

$-100.7$

$-46.2$

$-9.5$

7.2

28.2

30.2

20.0

17.7

19.0

16.7 
TOP FIELD (nT)

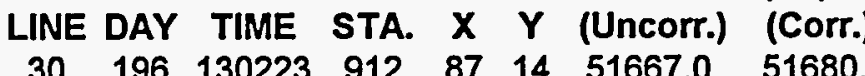

$30 \quad 196 \quad 130233 \quad 914$

$\begin{array}{lllll}30 & 196 & 130244 & 916\end{array}$

$\begin{array}{lllll}30 & 196 & 130256 & 918\end{array}$

$\begin{array}{lllll}30 & 196 & 130307 & 920\end{array}$

$\begin{array}{llll}30 & 196 & 130321 & 922\end{array}$

$\begin{array}{llll}30 & 196 & 130332 & 924\end{array}$

$30 \quad 196 \quad 130342 \quad 926$

$\begin{array}{llll}30 & 196 & 130353 & 928\end{array}$

$30 \quad 196 \quad 130404 \quad 930$

$\begin{array}{llll}30 & 196 & 130415 & 932\end{array}$

$30 \quad 196 \quad 130425 \quad 934$

$30 \quad 196 \quad 130436 \quad 936$

$\begin{array}{llll}30 & 196 & 130447 & 938\end{array}$

$\begin{array}{llll}30 & 196 & 130458 & 940\end{array}$

$\begin{array}{llll}31 & 196 & 131349 & 944\end{array}$

$\begin{array}{llll}31 & 196 & 131406 & 946\end{array}$

$\begin{array}{llll}31 & 196 & 131418 & 948\end{array}$

$\begin{array}{llll}31 & 196 & 131429 & 950\end{array}$

$\begin{array}{llll}31 & 196 & 131440 & 952\end{array}$

$\begin{array}{llll}31 & 196 & 131451 & 954\end{array}$

$\begin{array}{llll}31 & 196 & 131503 & 956\end{array}$

$\begin{array}{llll}31 & 196 & 131515 & 958\end{array}$

$\begin{array}{llll}31 & 196 & 131526 & 960\end{array}$

$\begin{array}{llll}31 & 196 & 131537 & 962\end{array}$

$\begin{array}{llll}31 & 196 & 131548 & 964\end{array}$

$\begin{array}{llll}31 & 196 & 131600 & 966\end{array}$

$\begin{array}{llll}31 & 196 & 131614 & 968\end{array}$

$\begin{array}{llll}31 & 196 & 131625 & 970\end{array}$

$\begin{array}{llll}31 & 196 & 131637 & 972\end{array}$

$\begin{array}{llll}31 & 196 & 131650 & 974\end{array}$

$\begin{array}{llll}31 & 196 & 131703 & 976\end{array}$

$\begin{array}{llll}31 & 196 & 131715 & 978\end{array}$

$\begin{array}{llll}31 & 196 & 131726 & 980\end{array}$

$\begin{array}{llll}31 & 196 & 131737 & 982\end{array}$

$\begin{array}{llll}31 & 196 & 131748 & 984\end{array}$

$\begin{array}{llll}31 & 196 & 131810 & 986\end{array}$

$\begin{array}{llll}31 & 196 & 131822 & 988\end{array}$

$\begin{array}{llll}31 & 196 & 131833 & 990\end{array}$

$\begin{array}{llll}31 & 196 & 131844 & 992\end{array}$

$\begin{array}{llll}31 & 196 & 131857 & 994\end{array}$

$\begin{array}{llll}31 & 196 & 131909 & 996\end{array}$

$\begin{array}{llll}31 & 196 & 131921 & 998\end{array}$

$\begin{array}{llll}31 & 196 & 131935 & 1000\end{array}$

$\begin{array}{llll}31 & 196 & 131946 & 1002\end{array}$

$\begin{array}{lllll}31 & 196 & 131958 & 1004\end{array}$

$\begin{array}{llll}31 & 196 & 132010 & 1006\end{array}$

$\begin{array}{llll}31 & 196 & 132022 & 1008\end{array}$

$31 \quad 196 \quad 132034 \quad 1010$ $\begin{array}{llllll}87 & 13 & 51671.0 & 51684.4 & -13.4\end{array}$

$\begin{array}{llllll}87 & 12 & 51674.8 & 51688.2 & -13.4\end{array}$

$\begin{array}{llllll}87 & 11 & 51677.4 & 51690.8 & -13.4\end{array}$

$\begin{array}{llllll}87 & 10 & 51676.4 & 51689.8 & -13.4\end{array}$

$\begin{array}{llllll}87 & 9 & 51676.4 & 51689.9 & -13.5\end{array}$

$\begin{array}{llllll}87 & 8 & 51675.8 & 51689.3 & -13.5\end{array}$

$\begin{array}{llllll}87 & 7 & 51675.2 & 51688.7 & -13.5\end{array}$

$\begin{array}{lll}87 & 6 & 51671.2\end{array}$

$\begin{array}{lll}87 & 5 & 51666.6\end{array}$

$\begin{array}{lll}87 & 4 & 51656.6\end{array}$

$\begin{array}{lll}87 & 3 & 51645.6\end{array}$

$\begin{array}{lll}87 & 2 & 51630.2\end{array}$

$\begin{array}{lll}87 & 1 & 51611.8\end{array}$

$\begin{array}{lll}87 & 0 & 51563.8\end{array}$

$\begin{array}{lll}90 & 0 & 51554.4\end{array}$

$90 \quad 1 \quad 51611.4$

$\begin{array}{lll}90 & 2 & 51638.4\end{array}$

903

904

905

906

907

908

909

$90 \quad 10$

9011

$\begin{array}{lll}90 & 12\end{array}$

$90 \quad 13$

$90 \quad 14$

$90 \quad 15$

$90 \quad 16$

$\begin{array}{lll}90 & 17\end{array}$

$\begin{array}{lll}90 & 18\end{array}$

$90 \quad 19$

9020

$90 \quad 21$

$90 \quad 22$

$90 \quad 23$

$90 \quad 24$

$90 \quad 25$

9026

$90 \quad 27$

$90 \quad 28$

$90 \quad 29$

$90 \quad 30$

$90 \quad 31$

$90 \quad 32$

$90 \quad 33$
51652.6

51662.8

51668.2

51673.2

51675.4

51677.8

51678.6

51678.6

51679.0

51677.4

51675.0

51671.4

51667.0

51661.2

51654.6

51647.2

51638.6

51629.4

51620.8

51617.8

51616.2

51625.0

51657.0

51683.6

51709.6

51732.6

51748.2

51745.6

51725.6

51699.8

51658.4
$51680.1-13.5$

$51670.1-13.5$

$51659.2-13.6$

$51643.8-13.6$

$51625.4-13.6$

$51577.4-13.6$

$51567.2-12.8$

$51624.3-12.9$

$51651.3-12.9$

$51665.5-12.9$

$51675.7-12.9$

$\begin{array}{ll}51681 & -12.8\end{array}$

$\begin{array}{lll}51686 & -12.8\end{array}$

$51688.2-12.8$

$51690.6-12.8$

$51691.4 \quad-12.8$

$\begin{array}{lll}51691.3 & -12.7\end{array}$

$51691.7-12.7$

$51690.1-12.7$

$\begin{array}{lll}51687.7 & -12.7\end{array}$

$51684-12.6$

$51679.6-12.6$

$51673.8-12.6$

$51667.2-12.6$

$51659.7-12.5$

$51651.1-12.5$

$51641.9-12.5$

$51633.2-12.4$

$51630.2-12.4$

$51628.6-12.4$

$51637.4-12.4$

$51669.4-12.4$

$51695.9-12.3$

$51721.9-12.3$

$51744.9-12.3$

$51760.5-12.3$

$51757.8-12.2$

$51737.8-12.2$

$51712-12.2$

$51670.6-12.2$
BOTTOM FIELD (nT)

GRAD.

$51684.7-13.5$

(Uncorr.) (Corr.) (Diff.)

(nT/m)

$\begin{array}{llll}51674.4 & 51686.6 & -12.2 & 12.1\end{array}$

$\begin{array}{llll}51678.8 & 51691.1 & -12.3 & 12.8\end{array}$

$\begin{array}{llll}51681.0 & 51693.3 & -12.3 & 10.2\end{array}$

$\begin{array}{llll}51682.2 & 51694.5 & -12.3 & 7.9\end{array}$

$\begin{array}{llll}51685.0 & 51697.3 & -12.3 & 14.1\end{array}$

$51674.2 \quad 51686.6 \quad-12.4 \quad-3.6$

$\begin{array}{llll}51677.6 & 51690.0 & -12.4 & 3.0\end{array}$

$51674.2 \quad 51686.6 \quad-12.4 \quad-1.6$

$\begin{array}{llll}51670.8 & 51683.2 & -12.4 & -0.7\end{array}$

$\begin{array}{llll}51660.6 & 51673.0 & -12.4 & -9.8\end{array}$

$\begin{array}{llll}51650.0 & 51662.5 & -12.5 & -10.8\end{array}$

$\begin{array}{llll}51634.6 & 51647.1 & -12.5 & -18.0\end{array}$

$\begin{array}{llll}51610.8 & 51623.3 & -12.5 & -31.8\end{array}$

$\begin{array}{lllll}51571.2 & 51583.7 & -12.5 & -66.6\end{array}$

$51488.6 \quad 51501.2 \quad-12.6 \quad-123.3$

$\begin{array}{lllll}51429.4 & 51441.4 & -12.0 & -204.9\end{array}$

$\begin{array}{llll}51561.4 & 51573.6 & -12.2 & -82.0\end{array}$

$\begin{array}{llll}51611.8 & 51624.0 & -12.2 & -43.6\end{array}$

$\begin{array}{llll}51633.6 & 51645.8 & -12.2 & -31.1\end{array}$

$\begin{array}{llll}51649.0 & 51661.1 & -12.1 & -22.6\end{array}$

$\begin{array}{llll}51657.8 & 51669.9 & -12.1 & -17.0\end{array}$

$51663.8 \quad 51675.9 \quad-12.1 \quad-15.4$

$51667.8 \quad 51679.9 \quad-12.1 \quad-12.5$

$\begin{array}{llll}51671.0 & 51683.1 & -12.1 & -11.1\end{array}$

$\begin{array}{llll}51672.8 & 51684.9 & -12.1 & -9.5\end{array}$

$\begin{array}{llll}51673.6 & 51685.6 & -12.0 & -8.2\end{array}$

$\begin{array}{llll}51674.0 & 51686.0 & -12.0 & -8.2\end{array}$

$\begin{array}{llll}51673.4 & 51685.4 & -12.0 & -6.6\end{array}$

$\begin{array}{llll}51671.6 & 51683.6 & -12.0 & -5.6\end{array}$

$\begin{array}{llll}51667.8 & 51679.8 & -12.0 & -5.9\end{array}$

$\begin{array}{llll}51662.6 & 51674.6 & -12.0 & -7.2\end{array}$

$\begin{array}{lllll}51658.4 & 51670.3 & -11.9 & -4.6\end{array}$

$\begin{array}{llll}51651.6 & 51663.5 & -11.9 & -4.9\end{array}$

$\begin{array}{llll}51643.2 & 51655.1 & -11.9 & -6.6\end{array}$

$\begin{array}{llll}51634.4 & 51646.3 & -11.9 & -6.9\end{array}$

$51623.2 \quad 51635.1 \quad-11.9 \quad-10.2$

$51612.6 \quad 51624.4 \quad-11.8 \quad-13.4$

$\begin{array}{llll}51604.0 & 51615.8 & -11.8 & -22.6\end{array}$

$\begin{array}{lllll}51593.8 & 51605.6 & -11.8 & -36.7\end{array}$

$\begin{array}{lllll}51565.2 & 51577.0 & -11.8 & -98.0\end{array}$

$\begin{array}{llll}51675.2 & 51687.0 & -11.8 & 29.8\end{array}$

$\begin{array}{llll}51658.0 & 51669.8 & -11.8 & -42.0\end{array}$

$51677.8 \quad 51689.6 \quad-11.8 \quad-52.1$

$\begin{array}{llll}51699.6 & 51711.3 & -11.7 & -54.1\end{array}$

$\begin{array}{lllll}51717.8 & 51729.5 & -11.7 & -49.8\end{array}$

$51709.6 \quad 51721.3 \quad-11.7 \quad-59.0$

$51683.6 \quad 51695.3 \quad-11.7 \quad-68.9$

$\begin{array}{llll}51670.0 & 51681.7 & -11.7 & -48.9\end{array}$

$\begin{array}{llll}51624.6 & 51636.2 & -11.6 & -55.4\end{array}$ 
TOP FIELD (nT)

LINE DAY TIME STA. $X Y$ (Uncorr.) (Corr.)

\section{1}

\section{1}

\section{1}

31

31

\section{1}

\section{1}

31

\section{1}

\section{1}

\section{3.}

\section{.}

\section{3.}

\section{3}

\section{.}

\section{3}

\section{3}

\section{3}

\section{3.}

31

\section{1}

\section{1}

\section{.}

.

3

\section{3}

\section{3}

3

\section{3}

\section{.}

\section{3}

\section{.}

\section{3}

\section{3}

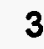

\section{3}

\section{3}

\section{3}

\section{3.}

\section{.}

31

\section{3.}

\section{3.} ${ }_{32}$ $\begin{array}{lllll}196 & 132047 & 1012 & 90 & 34\end{array}$ $\begin{array}{lllll}196 & 132058 & 1014 & 90 & 35\end{array}$ $\begin{array}{lllll}196 & 132112 & 1016 & 90 & 36\end{array}$ $\begin{array}{lllll}196 & 132125 & 1018 & 90 & 37\end{array}$ $\begin{array}{lllll}196 & 132138 & 1020 & 90 & 38\end{array}$ $\begin{array}{lllll}196 & 132151 & 1022 & 90 & 39\end{array}$ $\begin{array}{lllll}196 & 132205 & 1024 & 90 & 40\end{array}$ $\begin{array}{lllll}196 & 132216 & 1026 & 90 & 41\end{array}$ $\begin{array}{lllll}196 & 132231 & 1028 & 90 & 42\end{array}$ $\begin{array}{lllll}196 & 132245 & 1030 & 90 & 43\end{array}$ $\begin{array}{lllll}196 & 132303 & 1032 & 90 & 44\end{array}$ $\begin{array}{lllll}196 & 132315 & 1034 & 90 & 45\end{array}$ $\begin{array}{lllll}196 & 132327 & 1036 & 90 & 46\end{array}$ $\begin{array}{lllll}196 & 132339 & 1038 & 90 & 47\end{array}$

$$
51603.0
$$

51542.4

51530.4

51603.6

51690.8

51836.6

51937.8

51890.8

51781.8

51762.0

51925.8

52165.8 $\begin{array}{llllll}196 & 132351 & 1040 & 90 & 48 & 52173.8\end{array}$ $\begin{array}{llllll}196 & 132403 & 1042 & 90 & 49 & 51957.6\end{array}$ $\begin{array}{llllll}196 & 132414 & 1044 & 90 & 50 & 51701.8\end{array}$ $\begin{array}{llllll}196 & 132426 & 1046 & 90 & 51 & ? 51410\end{array}$ $\begin{array}{llllll}196 & 132439 & 1048 & 90 & 52 & ? 51203\end{array}$ $\begin{array}{llllll}196 & 132451 & 1050 & 90 & 53 & ? 51208.8\end{array}$ $\begin{array}{llllll}196 & 132504 & 1052 & 90 & 54 & ? 51214.8\end{array}$ $\begin{array}{llllll}196 & 132516 & 1054 & 90 & 55 & ? 51242.4\end{array}$ $\begin{array}{llllll}196 & 132530 & 1056 & 90 & 56 & ? 51749.8\end{array}$ $\begin{array}{llllll}196 & 132541 & 1058 & 90 & 57 & ? 52753.6\end{array}$ $\begin{array}{llllll}196 & 132554 & 1060 & 90 & 58 & ? 53765.8\end{array}$ $\begin{array}{llllll}196 & 132607 & 1062 & 90 & 59 & ? 53973.8\end{array}$ $\begin{array}{llllll}196 & 132624 & 1064 & 90 & 60 & ? 53314.2\end{array}$ $\begin{array}{llllll}196 & 132639 & 1066 & 90 & 61 & 52586.2\end{array}$ $\begin{array}{llllll}196 & 132651 & 1068 & 90 & 62 & ? 52194.2\end{array}$ $\begin{array}{llllll}196 & 132702 & 1070 & 90 & 63 & 52111.0\end{array}$ $\begin{array}{llllll}196 & 132713 & 1072 & 90 & 64 & 52022.2\end{array}$ $\begin{array}{llllll}196 & 132725 & 1074 & 90 & 65 & 51927.8\end{array}$ $\begin{array}{llllll}196 & 132738 & 1076 & 90 & 66 & 51864.8\end{array}$ $\begin{array}{llllll}196 & 132750 & 1078 & 90 & 67 & 51824.0\end{array}$ $\begin{array}{llllll}196 & 132801 & 1080 & 90 & 68 & 51792.2\end{array}$ $\begin{array}{llllll}196 & 132811 & 1082 & 90 & 69 & 51756.2\end{array}$ $\begin{array}{llllll}196 & 132822 & 1084 & 90 & 70 & 51718.6\end{array}$ $\begin{array}{llllll}196 & 132834 & 1086 & 90 & 71 & 51667.4\end{array}$ $\begin{array}{llllll}196 & 132846 & 1088 & 90 & 72 & 51555.0\end{array}$ $\begin{array}{llllll}196 & 132857 & 1090 & 90 & 73 & 51419.8\end{array}$ $\begin{array}{llllll}196 & 132909 & 1092 & 90 & 74 & ? 51512.8\end{array}$ $\begin{array}{llllll}196 & 132921 & 1094 & 90 & 75 & 51580.0\end{array}$ $\begin{array}{llllll}196 & 132933 & 1096 & 90 & 76 & 51623.4\end{array}$ $\begin{array}{llllll}196 & 132944 & 1098 & 90 & 77 & 51663.2\end{array}$ $\begin{array}{llllll}196 & 132955 & 1100 & 90 & 78 & 51682.0\end{array}$ $\begin{array}{llllll}196 & 133604 & 1102 & 93 & 72 & 51589.6\end{array}$ $\begin{array}{llllll}196 & 133616 & 1104 & 93 & 71 & 51684.8\end{array}$ $\begin{array}{llllll}196 & 133628 & 1106 & 93 & 70 & 51735.8\end{array}$ 32
51615.1 (Diffo)

$51554.5-12.1$

$51542.5-12.1$

$51615.7-12.1$

$51702.8-12.0$

$51848.6-12.0$

$51949.8-12.0$

$51902.8-12.0$

$51793.7-11.9$

$51773.9-11.9$

$51937.7-11.9$

$52177.6-11.8$

$52345-11.8$

$52353.4-11.8$

$52185.6-11.8$

$51969.4-11.8$

$51713.5-11.7$

$51421.7-11.7$

$51214.7-11.7$

$51220.5-11.7$

$51226.4-11.6$

$51254-11.6$

$51761.4-11.6$

$52765.2-11.6$

$53777.3-11.5$

$53985.3-11.5$

$53325.7-11.5$

$52597.6-11.4$

$52205.6-11.4$

$52122.4-11.4$

$52033.6-11.4$

$51939.2-11.4$

$51876.1-11.3$

$51835.3-11.3$

$51803.5-11.3$

$51767.5-11.3$

$51729.8-11.2$

$51678.6-11.2$

$51566.2-11.2$

$51431-11.2$

$51523.9-11.1$

$51591.1-11.1$

$51634.5-11.1$

$51674.3-11.1$

$51693.1-11.1$

$51599.9-10.3$

$51695.1-10.3$

$51746.1-10.3$

$51778.4-10.2$
BOTTOM FIELD (nT)

GRAD.

(Uncorr.) (Corr.) (Diff.) (nT/m)

$\begin{array}{lll}51531.4 & 51543.0 & -11.6\end{array}$

$\begin{array}{lll}51413.8 & 51425.4 & -11.6\end{array}$

$\begin{array}{llll}51419.8 & 51431.4 & -11.6\end{array}$

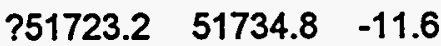

$\begin{array}{llll}51708.2 & 51719.8 & -11.6\end{array}$

$\begin{array}{llll}251981.4 & 51992.9 & -11.5\end{array}$

$\begin{array}{llll}252137.8 & 52149.3 & -11.5\end{array}$

$51954.0 \quad 51965.5 \quad-11.5$

$51696.0 \quad 51707.5 \quad-11.5$

?51545.6 $51557.1-11.5$

$51839.4 \quad 51850.8 \quad-11.4$

$52208.8 \quad 52220.2 \quad-11.4$

$\begin{array}{llll}252453.8 & 52465.2 & -11.4\end{array}$

?52530.4 $52541.8 \quad-11.4$

52262.0

52003.0

51656.0

?51114.8

?50612.2

51032.4

?51100.6

?50637

?51248.2

?52915.4

?54897.8

?58540.8

?53585.2

?52299.2

?51582.6

52000.6

51942.2

51865.6

51816.6

51786.4

51766.0

51737.8

51703.6

51655.8

51548.0

51329.6

$? 51283$

51427.6

51554.6

51641.6

51663.0

51613.4

51690.2

51731.6

51757.6

$52273.4 \quad-11.4$

$52014.4 \quad-11.4$

$51667.3 \quad-11.3$

$51126.1-11.3$

$50623.5-11.3$

$51043.7-11.3$

$51111.9-11.3$

$50648.3-11.3$

$51259.4-11.2$

$52926.6-11.2$

$54909.0-11.2$

$58552.0-11.2$

$\begin{array}{lll}53596.4 & -11.2\end{array}$

$52310.3-11.1$

$51593.7-11.1$

$52011.7-11.1$

$51953.3-11.1$

$51876.7-11$.

$51827.7-11.1$

$51797.4-11.0$

$\begin{array}{ll}51777.0 & -11.0\end{array}$

$\begin{array}{ll}51748.8 & -11.0\end{array}$

$51714.6-11.0$

$51666.8-11.0$

$51559.0-11.0$

$\begin{array}{lll}51340.5 & -10.9\end{array}$

$\begin{array}{ll}51293.9 & -10.9\end{array}$

$\begin{array}{ll}51438.5 & -10.9\end{array}$

$\begin{array}{ll}51565.5 & -10.9\end{array}$ 
TOP FIELD (nT)

LINE DAY TIME STA. $X Y$ (Uncorr.) (Corr.)

$\begin{array}{lllllll}32 & 196 & 133652 & 1110 & 93 & 68 & 51803.6\end{array}$

$\begin{array}{lllllll}32 & 196 & 133705 & 1112 & 93 & 67 & 51838.2\end{array}$

$\begin{array}{lllllll}32 & 196 & 133717 & 1114 & 93 & 66 & 51886.0\end{array}$

$\begin{array}{lllllll}32 & 196 & 133729 & 1116 & 93 & 65 & 51951.0\end{array}$

$\begin{array}{lllllll}32 & 196 & 133740 & 1118 & 93 & 64 & 52050.4\end{array}$

$\begin{array}{lllllll}32 & 196 & 133751 & 1120 & 93 & 63 & 52173.6\end{array}$

$\begin{array}{lllllll}32 & 196 & 133804 & 1122 & 93 & 62 & 52326.6\end{array}$

$\begin{array}{lllllll}32 & 196 & 133816 & 1124 & 93 & 61 & 52593.8\end{array}$

$\begin{array}{lllllll}32 & 196 & 133830 & 1126 & 93 & 60 & 52993.8\end{array}$

32

32

32

32

32

32

32

32

32

32

32

32

32

32

32

32

32

32

32

32

32

32

32

32

32

32

32

32

32

32

32

32

32

32

32

32

32

32

32

32 $\begin{array}{llllll}196 & 133845 & 1128 & 93 & 59 & ? 53281.6\end{array}$

$\begin{array}{llllll}196 & 133859 & 1130 & 93 & 58 & ? 53166.8\end{array}$

$\begin{array}{llllll}196 & 133912 & 1132 & 93 & 57 & ? 52372.6\end{array}$

$\begin{array}{llllll}196 & 133926 & 1134 & 93 & 56 & ? 51627.6\end{array}$

$\begin{array}{llllll}196 & 133938 & 1136 & 93 & 55 & ? 51233.8\end{array}$

$\begin{array}{llllll}196 & 133951 & 1138 & 93 & 54 & ? 51121.2\end{array}$

$\begin{array}{llllll}196 & 134004 & 1140 & 93 & 53 & ? 51122\end{array}$

$\begin{array}{llllll}196 & 134016 & 1142 & 93 & 52 & ? 51184\end{array}$

$\begin{array}{llllll}196 & 134031 & 1144 & 93 & 51 & 51333.6\end{array}$

$\begin{array}{llllll}196 & 134042 & 1146 & 93 & 50 & 51506.0\end{array}$

$\begin{array}{llllll}196 & 134054 & 1148 & 93 & 49 & 51670.6\end{array}$

$\begin{array}{llllll}196 & 134106 & 1150 & 93 & 48 & 51800.4\end{array}$

$\begin{array}{llllll}196 & 134117 & 1152 & 93 & 47 & 51865.0\end{array}$

$\begin{array}{lllllll}196 & 134129 & 1154 & 93 & 46 & 51890.6\end{array}$

$\begin{array}{lllllll}196 & 134140 & 1156 & 93 & 45 & 51890.2\end{array}$

$\begin{array}{llllll}196 & 134152 & 1158 & 93 & 44 & 51838.6\end{array}$

$\begin{array}{llllll}196 & 134204 & 1160 & 93 & 43 & 51794.0\end{array}$

$\begin{array}{llllll}196 & 134215 & 1162 & 93 & 42 & 51852.0\end{array}$

$\begin{array}{llllll}196 & 134226 & 1164 & 93 & 41 & 51926.4\end{array}$

$\begin{array}{llllll}196 & 134238 & 1166 & 93 & 40 & 51899.2\end{array}$

$\begin{array}{lllllll}196 & 134249 & 1168 & 93 & 39 & 51750.6\end{array}$

$\begin{array}{llllll}196 & 134301 & 1170 & 93 & 38 & 51568.0\end{array}$

$\begin{array}{llllll}196 & 134313 & 1172 & 93 & 37 & 51437.6\end{array}$

$\begin{array}{lllllll}196 & 134325 & 1174 & 93 & 36 & 51401.6\end{array}$

$\begin{array}{llllll}196 & 134336 & 1176 & 93 & 35 & 51443.2\end{array}$

$\begin{array}{lllllll}196 & 134348 & 1178 & 93 & 34 & 51502.4\end{array}$

$\begin{array}{llllll}196 & 134400 & 1180 & 93 & 33 & 51566.0\end{array}$

$\begin{array}{llllll}196 & 134419 & 1182 & 93 & 32 & 51603.4\end{array}$

$\begin{array}{llllll}196 & 134431 & 1184 & 93 & 31 & 51630.6\end{array}$

$\begin{array}{llllll}196 & 134442 & 1186 & 93 & 30 & 51648.2\end{array}$

$\begin{array}{llllll}196 & 134453 & 1188 & 93 & 29 & 51658.4\end{array}$

$\begin{array}{llllll}196 & 134506 & 1190 & 93 & 28 & 51662.6\end{array}$

$\begin{array}{llllll}196 & 134517 & 1192 & 93 & 27 & 51660.2\end{array}$

$\begin{array}{llllll}196 & 134529 & 1194 & 93 & 26 & 51663.8\end{array}$

$\begin{array}{lllllll}196 & 134540 & 1196 & 93 & 25 & 51658.6\end{array}$

$\begin{array}{llllll}196 & 134553 & 1198 & 93 & 24 & 51653.0\end{array}$

$\begin{array}{llllll}196 & 134605 & 1200 & 93 & 23 & 51651.0\end{array}$

$\begin{array}{llllll}196 & 134617 & 1202 & 93 & 22 & 51652.4\end{array}$

$\begin{array}{llllll}196 & 134629 & 1204 & 93 & 21 & 51653.6\end{array}$

$\begin{array}{llllll}196 & 134640 & 1206 & 93 & 20 & 51656.2\end{array}$
$51813.8-10.2$

$51848.4-10.2$

$51896.2-10.2$

$51961.2-10.2$

$52060.5-10.1$

$52183.7-10.1$

$52336.7-10.1$

$52603.9-10.1$

$53003.8-10.0$

$53291.6-10.0$

$53176.8-10.0$

$52382.6-10.0$

$51637.5-9.9$

$51243.7-9.9$

$51131.1-9.9$

$51131.8-9.8$

$51193.8-9.8$

$51343.4-9.8$

$51515.8-9.8$

$51680.3-9.7$

$\begin{array}{lll}51810.1 & -9.7\end{array}$

$51874.7-9.7$

$51900.3 \quad-9.7$

$51899.9-9.7$

$51848.2-9.6$

$51803.6-9.6$

$51861.6-9.6$

$51936-9.6$

$51908.7-9.5$

$51760.1-9.5$

$51577.5 \quad-9.5$

$51447.1-9.5$

$51411-9.4$

$51452.6-9.4$

$\begin{array}{lll}51511.8 & -9.4\end{array}$

$51575.4 \quad-9.4$

$51612.7-9.3$

$51639.9-9.3$

$51657.5-9.3$

$\begin{array}{lll}51667.7 & -9.3\end{array}$

$51671.8 \quad-9.2$

$51669.4-9.2$

$51673-9.2$

$51667.8-9.2$

$51662.2 \quad-9.2$

$51660.1-9.1$

$51661.5 \quad-9.1$

$51662.7-9.1$

$51665.3-9.1$
BOTTOM FIELD (nT)

GRAD.

(Uncorr.) (Corr.) (Diff.)

$\begin{array}{llll}51783.0 & 51793.3 & -10.3 & -33.8\end{array}$

$\begin{array}{lllll}51810.4 & 51820.7 & -10.3 & -45.6\end{array}$

$\begin{array}{llll}51847.0 & 51857.3 & -10.3 & -63.9\end{array}$

$\begin{array}{llll}51901.4 & 51911.6 & -10.2 & -81.3\end{array}$

$\begin{array}{lllll}51991.8 & 52002.0 & -10.2 & -96.1\end{array}$

$\begin{array}{llll}52105.0 & 52115.2 & -10.2 & -112.5\end{array}$

$\begin{array}{lllll}\text { ?52209.6 } & 52219.8 & -10.2 & -191.8\end{array}$

$\begin{array}{llll}52501.0 & 52511.2 & -10.2 & -152.1\end{array}$

$\begin{array}{lllll}252861.6 & 52871.8 & -10.2 & -216.7\end{array}$

$\begin{array}{lllll}253074 & 53084.1 & -10.1 & -340.3\end{array}$

$\begin{array}{lllll}? 53712.4 & 53722.5 & -10.1 & 894.4\end{array}$

$\begin{array}{lllll}? 52157 & 52167.1 & -10.1 & -353.4\end{array}$

$\begin{array}{lllll}? 51163.4 & 51173.5 & -10.1 & -761.0\end{array}$

$\begin{array}{llll}? 50920 & 50930.1 & -10.1 & -514.4\end{array}$

$? 50855 \quad 50865.0 \quad-10.0 \quad-436.4$

$? 50795 \quad 50805.0 \quad-10.0 \quad-536.1$

$\begin{array}{lllll}250834.6 & 50844.6 & -10.0 & -572.8\end{array}$

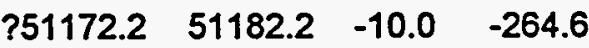

$\begin{array}{lllll}51442.2 & 51452.2 & -10.0 & -104.6\end{array}$

$\begin{array}{llll}51658.8 & 51668.7 & -9.9 & -19.3\end{array}$

$\begin{array}{llll}51812.4 & 51822.3 & -9.9 & 19.7\end{array}$

$\begin{array}{llll}51878.8 & 51888.7 & -9.9 & 22.6\end{array}$

?51826.8 $51836.7 \quad-9.9 \quad-104.6$

$\begin{array}{llll}51907.8 & 51917.7 & -9.9 & 28.9\end{array}$

$\begin{array}{llll}51815.4 & 51825.3 & -9.9 & -38.0\end{array}$

$\begin{array}{llll}251622.6 & 51632.5 & -9.9 & -281.0\end{array}$

$\begin{array}{llll}51829.0 & 51838.8 & -9.8 & -37.7\end{array}$

$\begin{array}{llll}52048.8 & 52058.6 & -9.8 & 200.7\end{array}$

$\begin{array}{llll}52003.8 & 52013.6 & -9.8 & 171.5\end{array}$

$\begin{array}{llll}51701.2 & 51711.0 & -9.8 & -81.0\end{array}$

$\begin{array}{lllll}251379.8 & 51389.6 & -9.8 & -308.5\end{array}$

$\begin{array}{lllll}251214.6 & 51224.4 & -9.8 & -365.6\end{array}$

$\begin{array}{lllll}251224.8 & 51234.5 & -9.7 & -289.8\end{array}$

$\begin{array}{lllll}51354.0 & 51363.7 & -9.7 & -146.2\end{array}$

$\begin{array}{llll}51450.0 & 51459.7 & -9.7 & -85.9\end{array}$

$\begin{array}{llll}51583.0 & 51592.7 & -9.7 & 27.9\end{array}$

$\begin{array}{llll}51597.2 & 51606.9 & -9.7 & -10.2\end{array}$

$\begin{array}{llll}51619.6 & 51629.2 & -9.6 & -18.0\end{array}$

$\begin{array}{llll}51641.6 & 51651.2 & -9.6 & -10.8\end{array}$

$51649.6 \quad 51659.2 \quad-9.6 \quad-14.4$

$51652.0 \quad 51661.6 \quad-9.6 \quad-17.4$

$\begin{array}{llll}51624.0 & 51633.6 & -9.6 & -59.3\end{array}$

$51658.0 \quad 51667.6 \quad-9.6 \quad-9.5$

$\begin{array}{llll}51656.0 & 51665.6 & -9.6 & -4.3\end{array}$

$51648.2 \quad 51657.7 \quad-9.5 \quad-7.9$

$51650.0 \quad 51659.5 \quad-9.5 \quad-1.6$

$51652.2 \quad 51661.7 \quad-9.5 \quad-0.3$

$\begin{array}{llll}51654.8 & 51664.3 & -9.5 & 2.0\end{array}$

$\begin{array}{llll}51661.6 & 51671.1 & -9.5 & 8.9\end{array}$ 
TOP FIELD (nT)

BOTTOM FIELD (nT)

GRAD.

\begin{tabular}{|c|c|c|c|c|c|c|c|c|c|c|c|c|}
\hline INE & AY & 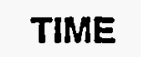 & A. & $x$ & $\mathbf{Y}$ & rr.) & T.) & Diff.) & rr.) & rr.) & (Diff.) & $T / m$ \\
\hline 32 & 196 & 134652 & 1208 & 93 & 19 & 51658.4 & 51667.4 & -9.0 & 51658.8 & 51668.3 & -9.5 & 0.7 \\
\hline 32 & 196 & 134704 & 1210 & 93 & 18 & 51662.0 & 51671 & -9.0 & 51666.2 & 51675.6 & -9.4 & 6.9 \\
\hline 32 & 196 & 134715 & 1212 & 93 & 17 & 51665.8 & 51674.8 & -9.0 & 51669.4 & 51678.8 & -9.4 & 5.9 \\
\hline 32 & 196 & 134729 & 1214 & 93 & 16 & 51669.4 & 51678.4 & -9.0 & 1672.2 & 51681.6 & -9.4 & 4.6 \\
\hline 32 & 196 & 134741 & 1216 & 93 & 15 & 51671.4 & 51680.3 & -8.9 & 51675.2 & 51684.6 & -9.4 & 6.2 \\
\hline 32 & 196 & 134753 & 1218 & 93 & 14 & 51674.4 & 51683.3 & -8.9 & 51676.6 & 51686.0 & -9.4 & 3.6 \\
\hline 32 & 196 & 134805 & 1220 & 93 & 13 & 51674.8 & 51683.7 & -8.9 & 77.2 & 51686.5 & -9.3 & 3.9 \\
\hline 32 & 196 & 134817 & 1222 & 93 & 12 & 51675.2 & 51684.1 & -8.9 & 51678.6 & 51687.9 & -9.3 & 5.6 \\
\hline 32 & 196 & 134828 & 1224 & 93 & 11 & 51674.6 & 51683.4 & -8.8 & 51679.2 & 51688.5 & -9.3 & 7.5 \\
\hline 32 & 196 & 134840 & 1226 & 93 & 10 & 51675.0 & 51683.8 & -8.8 & 51676.8 & 51686.1 & -9.3 & 3.0 \\
\hline 32 & 196 & 134851 & 1228 & 93 & 9 & 51673.0 & 51681.8 & -8.8 & 51674.8 & 51684.1 & -9.3 & 3.0 \\
\hline 32 & 196 & 134902 & 1230 & 93 & 8 & 51671.8 & 51680.6 & -8.8 & 51671.8 & 51681.1 & -9.3 & 0.0 \\
\hline 32 & 196 & 134913 & 1232 & 93 & 7 & 51 & 51677.4 & -8.8 & 69.4 & 51678.7 & -9.3 & 1.3 \\
\hline 32 & 196 & 134924 & 1234 & 93 & 6 & 51 & 51674.5 & -8.7 & 6.8 & 6.0 & -9.2 & 1.6 \\
\hline 32 & 196 & 36 & 1236 & 93 & 5 & 51 & 51670.7 & -8.7 & 2.0 & 1.2 & -9.2 & 0.0 \\
\hline 32 & 196 & 134947 & 1238 & 93 & 4 & 51 & 51666.7 & -8.7 & 2.2 & 1.4 & -9.2 & -9.5 \\
\hline 32 & 196 & 134959 & 1240 & 93 & 3 & 51 & 51661.1 & -8.7 & 44.2 & 51653.4 & -9.2 & -13.4 \\
\hline 32 & 196 & 135010 & 1242 & 93 & 2 & 516 & 51652.4 & -8.6 & 51631.2 & 51640.4 & -9.2 & -20.7 \\
\hline 32 & 196 & 135021 & 1244 & 93 & 1 & 516 & 51639.2 & -8.6 & 51607.8 & 51617.0 & -9.2 & -37.4 \\
\hline 32 & 196 & 135032 & 1246 & 93 & 0 & 51597.8 & 51606.4 & -8.6 & 46.4 & 51555.5 & -9.1 & -84.3 \\
\hline 33 & 196 & 141052 & 1250 & 96 & 0 & 51614.8 & 51621.4 & -6.6 & 51642.2 & 51649.0 & -6.8 & 44.9 \\
\hline 33 & 196 & 141105 & 1252 & 96 & 1 & 51626.6 & 51633 & -6.4 & 51605.6 & 51612.2 & -6.6 & -34.4 \\
\hline 33 & 196 & 141121 & 1254 & 96 & 2 & 516 & 51663 & -6.4 & 547 & 51553.6 & -6.6 & -179.7 \\
\hline 33 & 196 & 141223 & 1256 & 96 & 3 & 516 & 51644.9 & -6.3 & 32.6 & 51639.1 & -6.5 & -9.8 \\
\hline 33 & 196 & 141238 & 1258 & 96 & 4 & 51 & 51642.7 & -6.3 & 9.2 & 35.6 & -6.4 & -11.8 \\
\hline 33 & 196 & 141252 & 1260 & 96 & 5 & 51 & 51639.4 & -6.2 & 1.4 & 27.8 & -6.4 & -19.3 \\
\hline 33 & 196 & 141306 & 1262 & 96 & 6 & 51 & 51646.4 & -6.2 & 5.6 & 32.0 & -6.4 & -23.9 \\
\hline 33 & 196 & 141 & 1264 & 96 & 7 & 51 & 51660.4 & -6.2 & 2.2 & 48.5 & -6.3 & -19.7 \\
\hline 33 & 196 & 141 & 1266 & 96 & 8 & 51 & 1.6 & -6.2 & 1.8 & 58.1 & -6.3 & -22.3 \\
\hline 33 & 196 & 141348 & 1268 & 96 & 9 & 51 & 51681.7 & -6.1 & 9.8 & 76.1 & -6.3 & -9.5 \\
\hline 33 & 196 & 141400 & 1270 & 96 & 10 & 516 & 51688.1 & -6.1 & 6.8 & 51683.0 & -6.2 & -8.5 \\
\hline 33 & 196 & 141413 & 1272 & 96 & 11 & 516 & 51690.5 & -6.1 & 51675.8 & 51682.0 & -6.2 & -14.1 \\
\hline 33 & 196 & 141426 & 1274 & 96 & 12 & 51685.0 & 51691.1 & -6.1 & 7.0 & 51683.2 & -6.2 & -13.1 \\
\hline 33 & 196 & 141439 & 1276 & 96 & 13 & 51 & 51691.5 & -6.1 & 78.0 & 51684.2 & -6.2 & -12.1 \\
\hline 33 & 196 & 141452 & 1278 & 96 & 14 & 516 & 51691.6 & -6.0 & 8.8 & 84.9 & -6.1 & -11.1 \\
\hline 33 & 196 & 141503 & 1280 & 96 & 15 & 51 & 1.8 & -6.0 & 9.2 & 5.3 & -6.1 & -10.8 \\
\hline 33 & 196 & 141524 & 1282 & 96 & 16 & & & -6.0 & & 3.1 & -6.1 & -5.6 \\
\hline 33 & 196 & 141536 & 1284 & 96 & 17 & 51 & & -6.0 & 0.0 & 2.0 & -6.0 & -13.1 \\
\hline 33 & 196 & 141548 & 1286 & 96 & 18 & 516 & 51688.3 & -5.9 & 4.8 & 80.8 & -6.0 & -12.5 \\
\hline 33 & 196 & 141600 & 1288 & 96 & 19 & 51679.8 & 51685.7 & -5.9 & 51673.2 & 51679.2 & -6.0 & -10.8 \\
\hline 33 & 196 & 141611 & 1290 & 96 & 20 & 51679.2 & 51685.1 & -5.9 & 51671.6 & 51677.6 & -6.0 & -12.5 \\
\hline 33 & 196 & 141624 & 1292 & 96 & 21 & 51676.6 & 51682.5 & -5.9 & 51668.8 & 51674.7 & -5.9 & -12.8 \\
\hline 33 & 196 & 141635 & 1294 & 96 & 22 & 51674.8 & 51680.7 & -5.9 & 51667.2 & 51673.1 & -5.9 & -12.5 \\
\hline 33 & 196 & 141648 & 1296 & 96 & 23 & 51674.0 & 51679.8 & -5.8 & 51666.2 & 51672.1 & -5.9 & -12.8 \\
\hline 33 & 196 & 141700 & 1298 & 96 & 24 & 51672.8 & 51678.6 & -5.8 & 51662.6 & 51668.5 & -5.9 & -16.7 \\
\hline 33 & 196 & 141713 & 1300 & 96 & 25 & 516 & 51677.6 & -5.8 & 51659.8 & 51665.6 & -5.8 & -19.7 \\
\hline 33 & 196 & 141726 & 1302 & 96 & 26 & 51668.8 & 51674.6 & -5.8 & 51659.4 & 51665.2 & -5.8 & -15.4 \\
\hline 33 & 196 & 141738 & 1304 & 96 & 27 & 51665.4 & 51671.1 & -5.7 & 51658.2 & 51664.0 & -5.8 & -11.8 \\
\hline 33 & 196 & 141751 & 1306 & 96 & 28 & 51660.8 & 51666.5 & -5.7 & 51650.8 & 51656.6 & -5.8 & -16.4 \\
\hline
\end{tabular}


TOP FIELD (nT)

\begin{tabular}{|c|c|c|c|c|c|c|c|c|c|c|c|c|}
\hline & & & & & & & & (Diff.) & & 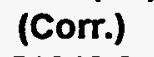 & (Diff.) & $(\mathrm{nT} / \mathrm{m})$ \\
\hline 33 & & a & 08 & & 29 & & 51655.3 & -5.7 & 51637.2 & 51642.9 & -5.7 & -20.3 \\
\hline 33 & 196 & 1816 & 1310 & 36 & 30 & 4.0 & 51639.7 & -5.7 & 51618.6 & 51624.3 & -5.7 & -25.2 \\
\hline 33 & 196 & 41828 & 312 & 96 & 31 & & 19.5 & -5.7 & & 1600.1 & -5.7 & -31.8 \\
\hline 33 & 196 & 141839 & 1314 & 96 & 32 & & 589 & 6 & 3.4 & 62.1 & 5.7 & -44.3 \\
\hline 33 & 196 & 141852 & 1316 & 36 & 33 & & 51546.6 & -5.6 & & 485.0 & -5.6 & -101.0 \\
\hline 33 & 196 & 141904 & 1318 & 6 & 34 & 7.2 & 51512.8 & -5.6 & 51362.4 & 368.0 & -5.6 & -237.4 \\
\hline 33 & 196 & 141916 & 1320 & 36 & 35 & & 51553.2 & -5.6 & 5.6 & 41.2 & -5.6 & -19.7 \\
\hline 33 & 196 & 141 & 1322 & 96 & 36 & & 51624.4 & -5.6 & & 1392.2 & -5.6 & -380.7 \\
\hline 33 & 96 & 45 & 1324 & 5 & 37 & & 84.5 & -5.5 & & 774.1 & -5.5 & -17.0 \\
\hline 33 & 196 & 41958 & 1326 & 6 & 38 & 8.6 & 52114.1 & -5.5 & 1.8 & 67.3 & -5.5 & 906.9 \\
\hline 33 & 196 & 42018 & 1328 & 6 & 39 & 7.4 & 52332.9 & -5.5 & & 75.4 & -5.4 & 1217.4 \\
\hline 33 & 196 & 42037 & 1330 & 6 & 40 & .6 & 52273 & -5.4 & & 10.2 & -5.4 & 602.0 \\
\hline 33 & 196 & 142052 & 1332 & 6 & 41 & & 52109.4 & -5.4 & $? 5$ & 5.2 & -5.4 & 304.6 \\
\hline 33 & 196 & 142 & 1334 & 96 & 42 & & 9.8 & -5.4 & & 14.1 & -5.3 & 7.2 \\
\hline 33 & 196 & 142 & 1336 & 96 & 43 & & 51758.4 & -5.4 & & 33.5 & -5.3 & -204.6 \\
\hline 33 & 196 & 29 & 1338 & 6 & 44 & & 51708.3 & -5.3 & 4.2 & 49.5 & -5.3 & -260.3 \\
\hline 33 & 96 & 41 & 1340 & 6 & 45 & & 0.1 & -5.3 & & 56.5 & -5.3 & -15.7 \\
\hline 33 & 196 & 53 & 1342 & 36 & 46 & & 2.3 & -5.3 & & 1.2 & -5.2 & 63.9 \\
\hline 33 & 196 & 06 & 1344 & 96 & 47 & & 3.7 & -5.3 & & 6.2 & -5.2 & 233.8 \\
\hline 33 & 196 & 19 & 1346 & 96 & 48 & & .1 & -5.3 & & 9.4 & -5.2 & 205.6 \\
\hline 33 & 196 & 142 & 1348 & 96 & 49 & & & -5.2 & & 3.0 & -5.2 & 121.0 \\
\hline 33 & 196 & 142 & 1350 & 96 & 50 & & & -5.2 & & 2.7 & 5.1 & 3.0 \\
\hline 33 & 196 & 142 & 1352 & 96 & 51 & & & -5.2 & & 2.7 & -5.1 & -273.1 \\
\hline 33 & 196 & 142 & 1354 & 96 & 52 & & 510 & -5.2 & & 6.7 & -5.1 & -823.6 \\
\hline 33 & 196 & 36 & 1356 & 6 & 53 & $? 5$ & 50 & -5.1 & & 9.6 & -5.0 & -421.0 \\
\hline 3. & 196 & 49 & 1358 & 6 & 54 & & 50 & -5.1 & & 8.2 & -5.0 & -967.9 \\
\hline 33 & 196 & 142 & 1360 & 6 & 55 & & & -5.1 & & 5.0 & -5.0 & -3220.3 \\
\hline 33 & 196 & 14 & 1362 & 96 & 56 & & & -5.1 & & 3.5 & -4.9 & 1122.6 \\
\hline 33 & 196 & 142 & 1364 & 96 & 57 & & & -5.0 & & & -4.9 & 724.3 \\
\hline 33 & 196 & 41 & 1366 & 96 & 58 & & & -5.0 & & & 4.9 & 026.9 \\
\hline 33 & 196 & 142454 & 1368 & 96 & 59 & & & -5.0 & & & -4.9 & 141.3 \\
\hline 33 & 196 & 142 & 1370 & 96 & 60 & & & -5.0 & & 4.2 & -4.8 & 411.8 \\
\hline 33 & 196 & 142 & 1372 & 96 & 61 & & 52763.1 & -4.9 & & 8.6 & -4.8 & -89.2 \\
\hline 33 & 196 & 32 & 1374 & 6 & 62 & 52 & 52372.5 & -4.5 & 3.8 & 8.6 & -4.8 & -153.8 \\
\hline 33 & 196 & & 1376 & 6 & 63 & & 52 & -4 & & 4.8 & -4.8 & -194.8 \\
\hline 33 & 196 & & 1378 & 96 & 64 & & 52 & -4.9 & & 0.5 & -4.7 & -116.4 \\
\hline 33 & 196 & & 1380 & 96 & 65 & & & -4.9 & & 3.3 & -4.7 & -98.0 \\
\hline 33 & 196 & 142 & 1382 & 96 & 66 & & & -4.8 & & & -4.7 & -73.4 \\
\hline 33 & 196 & 142631 & 1384 & 96 & 67 & & & -4.8 & & 7.9 & -4.7 & -62.3 \\
\hline 33 & 196 & 42 & 1386 & 96 & 68 & & 12.6 & -4.8 & & 4.8 & -4.6 & -45.2 \\
\hline 33 & 19 & & 1388 & 96 & 69 & & & -4.8 & & 0.6 & -4.6 & -34.4 \\
\hline 33 & 19 & & 1390 & 96 & 70 & & & -4.8 & & 1.6 & -4.6 & -23.6 \\
\hline 33 & 196 & 142721 & 1392 & 96 & 71 & & 51 & -4.7 & & 30.4 & -4.6 & -10.2 \\
\hline 33 & 196 & 142738 & 1394 & 96 & 72 & & & -4.7 & & & -4.5 & 3.6 \\
\hline 34 & 196 & 150304 & 1396 & 99 & 71 & & 51671.2 & -1.0 & & & & 21.0 \\
\hline 34 & 196 & 150318 & 1398 & 99 & 70 & & & -1.0 & & 29.4 & 0.0 & 15.4 \\
\hline 34 & 196 & 150332 & 1400 & 99 & 69 & & 51761 & -1.0 & & 58.8 & 0.0 & -2.0 \\
\hline 34 & 19 & 150349 & 1402 & 99 & 68 & & 51791.7 & -0.9 & 84.4 & 784.3 & 0.1 & -10.5 \\
\hline 34 & 196 & 150402 & 1404 & 99 & 67 & 51818.4 & 51819.3 & -0.9 & 51807.4 & 51807.3 & 0.1 & -18.0 \\
\hline
\end{tabular}


TOP FIELD (nT)

BOTTOM FIELD (nT)

GRAD.

\begin{tabular}{|c|c|c|c|c|c|c|c|c|c|c|c|c|}
\hline 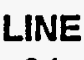 & DAY & 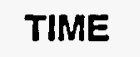 & A. & 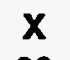 & & & 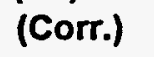 & (Diff.) & rr.) & orr.) & (Diff.) & (nT/m) \\
\hline 34 & 196 & 150418 & 1406 & 99 & 66 & 51855.6 & 51856.5 & -0.9 & 51830.0 & 51829.9 & 0.1 & -42.0 \\
\hline 34 & 196 & 150432 & 1408 & 99 & 65 & 51901.0 & 51901.8 & -0.8 & 51866.0 & 51865.8 & 0.2 & -57.4 \\
\hline 34 & 196 & 0446 & 1410 & 99 & 64 & 51967.8 & 51968.6 & -0.8 & 4.0 & 1913.8 & 0.2 & -88.2 \\
\hline 34 & 196 & 00 & 1412 & 99 & 63 & 52065.2 & 52066 & -0.8 & 8.0 & & 0.2 & 10.2 \\
\hline 34 & 196 & 150516 & 1414 & 99 & 62 & 52256.6 & 52257.4 & -0.8 & & 3.5 & 0.3 & 168.5 \\
\hline 34 & 196 & 150533 & 1416 & 99 & 61 & 52644.4 & 52645.1 & -0.7 & ?52543.6 & 2543.3 & 0.3 & -165.2 \\
\hline 34 & 196 & 150608 & 1418 & 99 & 60 & ?53337 & 53337.7 & -0.7 & ?53587 & 53586.6 & 0.4 & 409.8 \\
\hline 34 & 196 & 150628 & 1420 & 99 & 59 & ?53727 & 53727.6 & -0.6 & $? 58565.2$ & 58564.8 & 0.4 & 7931.5 \\
\hline 34 & 196 & 150653 & 1422 & 99 & 58 & ?53559.2 & 53559.8 & -0.6 & 643 & 642.5 & 0.5 & 776.7 \\
\hline 34 & 196 & 150708 & 1424 & 99 & 57 & ?52852.4 & 52853 & -0.6 & 12.6 & 3012.1 & 0.5 & 262.6 \\
\hline 34 & 196 & 150722 & 1426 & 99 & 56 & ?52150 & 52150.6 & -0.6 & ?51963.6 & 63.1 & 0.5 & 305.6 \\
\hline 34 & 196 & 35 & 1428 & 99 & 55 & 3.8 & 54.3 & -0.5 & 7.8 & 7.3 & 0.5 & 316.4 \\
\hline 34 & 196 & 150748 & 1430 & 99 & 54 & 3.2 & 51283.7 & -0.5 & & 9.4 & 0.6 & -316.7 \\
\hline 34 & 196 & 150801 & 1432 & 99 & 53 & $? 51162.8$ & 51163.3 & -0.5 & 8.8 & 18.2 & 0.6 & -563.9 \\
\hline 34 & 196 & 150815 & 1434 & 99 & 52 & & 51185.5 & -0.5 & & 38.2 & 0.6 & -469.2 \\
\hline 34 & 196 & 150828 & 1436 & 99 & 51 & 513 & 51350.4 & -0.4 & 55.8 & 55.1 & 0.7 & 9.5 \\
\hline 34 & 196 & 150841 & 1438 & 99 & 50 & 51466.6 & 51467 & -0.4 & 4.6 & 83.9 & 0.7 & 134.4 \\
\hline 34 & 196 & 150853 & 1440 & 99 & 49 & $51 !$ & 51559.4 & -0.4 & .2 & 3.5 & 0.7 & -155.4 \\
\hline 34 & 196 & 150907 & 1442 & 99 & 48 & 51 & 51692 & -0.4 & 6 & .9 & 0.7 & -55.7 \\
\hline 34 & 196 & 150920 & 1444 & 99 & 47 & 518 & 51839.9 & -0.3 & & 0.6 & 0.8 & 199.7 \\
\hline 34 & 196 & 150939 & 1446 & 99 & 46 & 519 & 32.9 & -0.3 & .2 & 3.4 & 0.8 & 428.8 \\
\hline 34 & 196 & 150953 & 1448 & 99 & 45 & 51 & 51834.1 & -0.3 & .2 & 4.4 & 0.8 & 313.8 \\
\hline 34 & 196 & 151006 & 1450 & 99 & 44 & 517 & 51706.1 & -0.3 & & 51.1 & 0.9 & -88.2 \\
\hline 34 & 196 & 151019 & 1452 & 99 & 43 & 516 & 51675 & -0.2 & & 6.7 & 0.9 & -208.5 \\
\hline 34 & 196 & 151033 & 1454 & 99 & 42 & 517 & 51760.2 & -0.2 & 517 & 25.7 & 0.9 & -54.8 \\
\hline 34 & 196 & 151046 & 1456 & 99 & 41 & 51 & 51864.8 & -0.2 & .2 & 5.3 & 0.9 & 133.8 \\
\hline 34 & 196 & 151100 & 1458 & 99 & 40 & 519 & 51911.8 & -0.2 & $? 52$ & 5.6 & 1.0 & 254.1 \\
\hline 34 & 196 & 151113 & 1460 & 99 & 39 & 518 & 51851.5 & -0.1 & .4 & 1.4 & 1.0 & 50.8 \\
\hline 34 & 196 & 151126 & 1462 & 99 & 38 & 517 & 51736.3 & -0.1 & & .6 & 1.0 & -23.9 \\
\hline 34 & 196 & 151138 & 1464 & 99 & 37 & 516 & 51642.7 & -0.1 & & 0.5 & 1.1 & -139.3 \\
\hline 34 & 196 & 151151 & 1466 & 99 & 36 & & 9.9 & -0.1 & & 1.1 & 1.1 & -160.0 \\
\hline 34 & 196 & 151204 & 1468 & 99 & 35 & & 5.9 & -0.1 & & 2.3 & 1.1 & -135.1 \\
\hline 34 & 196 & 151218 & 1470 & 99 & 34 & $51 !$ & 42.2 & 0.0 & .2 & 36.1 & 1.1 & -73.8 \\
\hline 34 & 196 & 151232 & 1472 & 99 & 33 & 515 & 51567.4 & 0.0 & 0.2 & 49.0 & 1.2 & -28.2 \\
\hline 34 & 196 & 151244 & 1474 & 99 & 32 & 515 & 593.8 & 0.0 & .2 & 34.0 & 1.2 & 2.3 \\
\hline 34 & 196 & 151257 & 1476 & 99 & 31 & 516 & 51616.8 & 0.0 & .8 & 6.6 & 1.2 & 1.6 \\
\hline 34 & 196 & 151309 & 1478 & 99 & 30 & 516 & 0.1 & 0.1 & & 3.7 & 1.3 & -24.9 \\
\hline 34 & 196 & 151322 & 1480 & 99 & 29 & & 3.9 & 0.1 & & & 1.3 & -2.3 \\
\hline 34 & 196 & & 1482 & 99 & 28 & & 6.7 & 0.1 & & 3.9 & 1.3 & 5.6 \\
\hline 34 & 196 & 151348 & 1484 & 99 & 27 & 516 & 66.7 & 0.1 & & 9.7 & 1.3 & 6.9 \\
\hline 34 & 196 & 151401 & 1486 & 99 & 26 & 516 & 51672.1 & 0.1 & 9.8 & 78.4 & 1.4 & 12.5 \\
\hline 34 & 196 & 151414 & 1488 & 99 & 25 & 516 & 51675.8 & 0.2 & 9.8 & 8.4 & 1.4 & 6.2 \\
\hline 34 & 196 & 151426 & 1490 & 99 & 24 & 516 & 51678.6 & 0.2 & .0 & 3.6 & 1.4 & 10.2 \\
\hline 34 & 196 & 151438 & 1492 & 99 & 23 & 516 & 880 & 0.2 & .4 & 1.0 & 1.4 & 3.6 \\
\hline 34 & 196 & 151450 & 1494 & 99 & 22 & 516 & 51680.4 & 0.2 & & 6.3 & 1.5 & 11.8 \\
\hline 34 & 196 & 151502 & 1496 & 99 & 21 & 516 & 51682 & 0.2 & & 7.9 & 1.5 & 11.8 \\
\hline 34 & 196 & 151513 & 1498 & 99 & 20 & 51683.6 & 51683.3 & 0.3 & & 51687.3 & 1.5 & 8.5 \\
\hline 34 & 196 & 151525 & 1500 & 99 & 19 & 51684.8 & 51684.5 & 0.3 & & 51688.5 & 1.5 & 8.5 \\
\hline 34 & 196 & 151538 & 1502 & 99 & 18 & 51686.0 & 51685.7 & 0.3 & 51691.6 & 51690.0 & 1.6 & 9.2 \\
\hline
\end{tabular}


TOP FIELD (nT)

\begin{tabular}{|c|c|c|c|c|c|c|c|c|c|c|c|c|}
\hline 24 & DAY & TII & A. & $X$ & 17 & (Uncorr.) & (Corr.) & (Diff.) & (Uncorr.) & $\begin{array}{l}\text { (Corr.) } \\
516928\end{array}$ & (Diff.) & (nT/m) \\
\hline 34 & 196 & 550 & & 99 & 17 & & 51686.1 & 0.3 & 51694.4 & 51692.8 & 1.6 & 13.1 \\
\hline 34 & 196 & 151602 & 1506 & 99 & 16 & 51687.0 & 51686.6 & 0.4 & 51693.6 & 51692.0 & 1.6 & 10.8 \\
\hline 34 & 196 & 151614 & 1508 & 99 & 15 & 51687.4 & 51687 & 0.4 & 691.8 & 590.2 & 1.6 & .2 \\
\hline 34 & 196 & & 1510 & 99 & 14 & 88.0 & 51687.6 & 0.4 & 694.4 & 1692.7 & 1.7 & 10.5 \\
\hline 34 & 196 & 151639 & 1512 & 99 & 13 & 51686.8 & 51686.4 & 0.4 & 51692.6 & 51690.9 & 1.7 & 9.5 \\
\hline 34 & 196 & 151651 & 1514 & 99 & 12 & 51686.2 & 51685.8 & 0.4 & 51692.8 & 51691.1 & 1.7 & 10.8 \\
\hline 34 & 196 & 151702 & 1516 & 99 & 11 & 51685.2 & 51684.7 & 0.5 & 51690.0 & 51688.3 & 1.7 & 7.9 \\
\hline 34 & 196 & 151713 & 1518 & 99 & 10 & 51684.2 & 51683.7 & 0.5 & 51686.6 & 51684.8 & 1.8 & 3.9 \\
\hline 34 & 196 & 151726 & 1520 & 99 & 9 & 51681.6 & 51681.1 & 0.5 & 51687.2 & 51685.4 & 1.8 & 9.2 \\
\hline 34 & 196 & 151750 & 1522 & 99 & 8 & 51687.2 & 51686.7 & 0.5 & 51670.2 & 51668.4 & 1.8 & -27.9 \\
\hline 34 & 196 & 151824 & 1524 & 99 & 7 & 1714.4 & 51713.8 & 0.6 & 51675.6 & 51673.7 & 1.9 & -63.6 \\
\hline 34 & 196 & 151842 & 1526 & 99 & 6 & 51904.2 & 51903.6 & 0.6 & ?51780.2 & 51778.2 & 2.0 & -203.3 \\
\hline 34 & 196 & 151857 & 1528 & 99 & 5 & 51.8 & 51.1 & 0.7 & 3.4 & 94.4 & 2.0 & 5828.5 \\
\hline 34 & 196 & 151916 & 1530 & 99 & 4 & $? 51849.4$ & 51848.7 & 0.7 & 1.8 & 51149.8 & 2.0 & -1143.6 \\
\hline 34 & 196 & 151931 & 1532 & 99 & 3 & 51586.6 & 51585.9 & 0.7 & 9.2 & 51447.1 & 2.1 & -225.3 \\
\hline 34 & 196 & 151944 & 1534 & 99 & 2 & 51585.0 & 51584.3 & 0.7 & 9.4 & 51547.3 & 2.1 & -58.4 \\
\hline 34 & 196 & 151959 & 1536 & 99 & 1 & 51569.0 & 51568.2 & 0.8 & 51526.6 & 51524.5 & 2.1 & -69.5 \\
\hline 34 & 196 & 152013 & 1538 & 99 & 0 & 51501.6 & 51500.8 & 0.8 & 51403.2 & 51401.1 & 2.1 & -161.3 \\
\hline 35 & 196 & 152428 & 1542 & 102 & 0 & ?51443 & 51442.5 & 0.5 & 512 & 51266.1 & 2.1 & -286.6 \\
\hline 35 & 196 & 152445 & 1544 & 102 & 1 & 51547.4 & 51546.7 & 0.7 & 514 & 51465.0 & 2.4 & -131.1 \\
\hline 35 & 196 & 152457 & 1546 & 102 & 2 & 51597.8 & 51597.1 & 0.7 & 5.4 & 51553.1 & 2.3 & -69.5 \\
\hline 35 & 196 & 152512 & 1548 & 102 & 3 & 51630.6 & 51630 & 0.6 & .2 & 51593.9 & 2.3 & -56.4 \\
\hline 35 & 196 & 152530 & 1550 & 102 & 4 & 48.4 & 51647.8 & 0.6 & 0.8 & 4.5 & 2.3 & -84.6 \\
\hline 35 & 196 & 152545 & 1552 & 102 & 5 & 51667.0 & 51666.4 & 0.6 & $? 5$ & 1.1 & 2.3 & -104.3 \\
\hline 35 & 196 & 152 & 1554 & 102 & 6 & 51679.4 & 51678.9 & 0.5 & & 50.1 & 2.3 & -44.3 \\
\hline 35 & 196 & 152624 & 1556 & 102 & 7 & 51 & 51682.7 & 0.5 & 6.2 & 53.9 & 2.3 & -44.3 \\
\hline 35 & 196 & 152641 & 1558 & 102 & 8 & 51684.6 & 51684.1 & 0.5 & 1.0 & 51668.7 & 2.3 & -22.3 \\
\hline 35 & 196 & 152654 & 1560 & 102 & 9 & 51684.2 & 51683.8 & 0.4 & 9.8 & 51667.5 & 2.3 & -23.6 \\
\hline 35 & 196 & 152707 & 1562 & 102 & 10 & $? 51671.8$ & 51671.4 & 0.4 & 9.4 & 51667.1 & 2.3 & -3.9 \\
\hline 35 & 196 & 152722 & 1564 & 102 & 11 & ?51677.4 & 51677 & 0.4 & 78.8 & 51676.5 & 2.3 & 2.3 \\
\hline 35 & 196 & 152801 & 1566 & 102 & 12 & 51688.0 & 51687.7 & 0.3 & 8.4 & 51676.1 & 2.3 & -15.7 \\
\hline 35 & 196 & 152813 & 1568 & 102 & 13 & 51686.2 & 51685.9 & 0.3 & 51679.4 & 51677.1 & 2.3 & -11.1 \\
\hline 35 & 196 & 152826 & 1570 & 102 & 14 & 51687.2 & 51687 & 0.2 & $? 51681.6$ & 51679.3 & 2.3 & -9.2 \\
\hline 35 & 196 & 152838 & 1572 & 102 & 15 & 51686.2 & 51686 & 0.2 & 3.2 & 51675.9 & 2.3 & -13.1 \\
\hline 35 & 196 & 152850 & 1574 & 102 & 16 & 51686.0 & 51685.8 & 0.2 & .8 & 4.5 & 2.3 & -15.1 \\
\hline 35 & 196 & 152902 & 1576 & 102 & 17 & ?51674.2 & 51674 & 0.2 & & 5.3 & 2.3 & 5. \\
\hline 35 & 196 & 152915 & 1578 & 102 & 18 & 51686.8 & 51686.7 & 0.1 & 6.8 & 74.5 & 2.3 & -16.4 \\
\hline 35 & 196 & 152927 & 1580 & 102 & 19 & 51688.6 & 51688.5 & 0.1 & 4.6 & 51672.3 & 2.3 & -23.0 \\
\hline 35 & 196 & 152939 & 1582 & 102 & 20 & 51686.6 & 51686.5 & 0.1 & 51672.8 & 51670.5 & 2.3 & -22.6 \\
\hline 35 & 196 & 152952 & 1584 & 102 & 21 & $? 51679$ & 51678.9 & 0.1 & 51689.4 & 51687.1 & 2.3 & 17.1 \\
\hline 35 & 196 & 153008 & 1586 & 102 & 22 & ?51678.8 & 51678.8 & 0.0 & $? 51682.2$ & 51680.0 & 2.2 & 5.6 \\
\hline 35 & 196 & 153021 & 1588 & 102 & 23 & ?51673.8 & 51673.8 & 0.0 & ?51680.6 & 51678.4 & 2.2 & 11.1 \\
\hline 35 & 196 & 153034 & 1590 & 102 & 24 & 51685.6 & 51685.6 & 0.0 & 3.0 & 51670.8 & 2.2 & -20.7 \\
\hline 35 & 196 & 153047 & 1592 & 102 & 25 & 51683.8 & 51683.8 & 0.0 & 1.6 & 51679.4 & 2.2 & -3.6 \\
\hline 35 & 196 & 153059 & 1594 & 102 & 26 & 51680.6 & 51680.7 & -0.1 & .6 & 51672.4 & 2.2 & -9.8 \\
\hline 35 & 196 & 153113 & 1596 & 102 & 27 & 51673.0 & 51673.1 & -0.1 & 51666.0 & 51663.8 & 2.2 & -11.5 \\
\hline 35 & 196 & 153128 & 1598 & 102 & 28 & 51664.8 & 51664.9 & -0.1 & 51652.2 & 51650.0 & 2.2 & -20.7 \\
\hline 35 & 196 & 153141 & 1600 & 102 & 29 & 51655.6 & 51655.7 & -0.1 & 51643.4 & 51641.2 & 2.2 & -20.0 \\
\hline 35 & 196 & 153153 & 1602 & 102 & 30 & 51646.4 & 51646.6 & -0.2 & 51640.6 & 51638.4 & 2.2 & -9.5 \\
\hline
\end{tabular}

BOTTOM FIELD (nT) GRAD. 10 .5 9 3.9 $-27.9$ 03.3 143.6 $-225.3$ $-58.4$ $-69.5$ $-161.3$ $-286.6$ $-131.1$ $-69.5$ $-56.4$ $-84.6$ $-104.3$ $-44.3$ 2.3 $-3.9$ $-15.7$ (1.1 $-13.1$

- 6.4 $-22.6$ 6 1.1 $-9.8$ 207 $-9.5$ 
TOP FIELD (nT)

LINE DAY TIME STA $X Y$ (Uncorr) (Corr)
BOTTOM FIELD (nT) GRAD.

(nT/m)

13.8

$-23.3$

$-109.5$

455.4

401.3

$-337.1$

$-24.6$

$-56.7$

25.9

$-36.4$

$-169.5$

$-96.4$

$-361.0$

319.3

$-486.6$

$-121.6$

$-120.0$

$-197.0$

27.5

02.6

076.1

803.0

$-185.9$

$-264.9$

$-128.2$

$-92.5$

69.8

$-58.0$

$-48.5$

$-37.4$

29.2

$-18.4$

31.1

59.3

349.8

$-483.9$

$-236.4$

$-41.0$

$-65.2$ 
TOP FIELD (nT)

BOTTOM FIELD (nT)

GRAD.

\begin{tabular}{|c|c|c|c|c|c|c|c|c|c|c|c|c|}
\hline & & & & & & & (Corr.) & 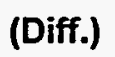 & orr.) & :orr.) & (Diff.) & 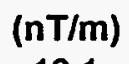 \\
\hline 36 & & & 702 & 105 & 68 & 1743.2 & 51745.4 & -2.2 & 51753.0 & 51751.1 & 1.9 & 16.1 \\
\hline 36 & 196 & 154907 & 1704 & 105 & 67 & 71.8 & 51774.1 & -2.3 & 51768.8 & 1766.9 & 1.9 & -4.9 \\
\hline 36 & 196 & 154917 & 1706 & 105 & 66 & 792.0 & 51794.3 & -2.3 & 51782.4 & 5 & 1.9 & -15.7 \\
\hline 36 & 196 & 154928 & 1708 & 105 & 65 & 51818.0 & 51820.3 & -2.3 & 51803.4 & 801.5 & 1.9 & -23.9 \\
\hline 36 & 196 & 154939 & 1710 & 105 & 64 & 51849.8 & 51852.1 & -2.3 & 51826.8 & 1824.9 & 1.9 & -37.7 \\
\hline 36 & 196 & 154951 & 1712 & 105 & 63 & 51894.8 & 51897.2 & -2.4 & 51857.8 & 1855.9 & 1.9 & -60.7 \\
\hline 36 & 196 & 155002 & 1714 & 105 & 62 & 51961.6 & 51964 & -2.4 & 51917.8 & 1915.9 & 1.9 & -71.8 \\
\hline 36 & 196 & 155014 & 1716 & 105 & 61 & 52066.8 & 52069.2 & -2.4 & 52031.6 & 2029.7 & 1.9 & -57.7 \\
\hline 36 & 196 & 155029 & 1718 & ○ & 60 & 52241.0 & 52243.4 & -2.4 & ?52166.8 & 64.9 & 1.9 & -121.6 \\
\hline 36 & 196 & 155040 & 1720 & 105 & 99 & 52523.8 & 52526.3 & -2.5 & 7.0 & 05.2 & 1.8 & 36.4 \\
\hline 36 & 196 & 155052 & 1722 & 105 & 58 & ?52810.6 & 52813.1 & -2.5 & & 63.2 & 1.8 & 238.7 \\
\hline 36 & 196 & 155106 & 1724 & 105 & 57 & $? 52775$ & 52777.5 & -2.5 & 4.2 & 52382.4 & 1.8 & 640.7 \\
\hline 36 & 196 & 155118 & 1726 & 105 & 56 & 52479.4 & 52481.9 & -2.5 & 2.2 & 52580.4 & 1.8 & 168.5 \\
\hline 36 & 196 & 155129 & 1728 & 105 & 55 & 52187.6 & 52190.2 & -2.6 & 522 & 34.4 & 1.8 & 79.7 \\
\hline 36 & 196 & 155141 & 1730 & 105 & 54 & 51925.6 & 51928.2 & -2.6 & 3.6 & 51711.8 & 1.8 & 47.5 \\
\hline 36 & 196 & 54 & 1732 & 105 & 53 & 51 & 51772.2 & -2.6 & 9.6 & 27.8 & 1.8 & 393.4 \\
\hline 36 & 196 & 155 & 1734 & 105 & 2 & 5 & 13.4 & -2.6 & 4.2 & 2.4 & .8 & -387.9 \\
\hline 36 & 196 & 155 & 1736 & 105 & 1 & 5 & 3.4 & -2.6 & & 0.8 & 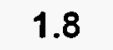 & 3.0 \\
\hline 36 & 196 & 155227 & 1738 & 105 & 50 & 5 & 33.7 & -2.7 & & 3.4 & .8 & 70.8 \\
\hline 36 & 196 & 155238 & 1740 & 105 & 49 & 51 & 51888.7 & -2.7 & & 1.2 & .8 & -4.9 \\
\hline 36 & 196 & 155249 & 1742 & 105 & 48 & & 51992.7 & -2.7 & & 12.4 & 1.8 & 03.6 \\
\hline 36 & 196 & 155300 & 1744 & 105 & 47 & $52 C$ & 52090.7 & -2.7 & & 04.8 & .0 & 58.4 \\
\hline 36 & 196 & 155312 & 1746 & 105 & 46 & 52142.2 & 52145 & -2.8 & 0.6 & 58.8 & 1.8 & 58.0 \\
\hline 36 & 196 & 155323 & 1748 & 105 & 45 & 52 & 52116.4 & -2.8 & .8 & 68.0 & .8 & 256.1 \\
\hline 36 & 196 & 155334 & 1750 & 105 & 44 & 520 & 52007.8 & -2.8 & .6 & 0.8 & .8 & 60.0 \\
\hline 36 & 196 & 155345 & 1752 & 10 & 43 & 51 & 51855 & -2.8 & .8 & 5.0 & .8 & 140.0 \\
\hline 36 & 196 & 155356 & 1754 & 105 & 42 & 51 & 51806.5 & -2.9 & & 1.0 & 1.8 & -148.9 \\
\hline 36 & 196 & 15 & 1756 & 105 & 41 & 5 & 28.1 & -2.9 & .8 & 1.0 & .8 & -3.9 \\
\hline 36 & 196 & 155 & 1758 & 105 & 40 & 51 & 1.9 & -2.9 & & & .8 & 3.0 \\
\hline 36 & 196 & 155433 & 1760 & 105 & 39 & 51 & 51855.5 & -2.9 & & 5.0 & .8 & 3.3 \\
\hline 36 & 196 & 155446 & 1762 & 105 & 38 & $51 \varepsilon$ & 51806 & -3.0 & 9.2 & 7.4 & 1.8 & 186.6 \\
\hline 36 & 196 & 155457 & 1764 & 105 & 37 & 51769.2 & 51772.2 & -3.0 & 1.2 & 09.4 & 1.8 & -259.0 \\
\hline 36 & 196 & 155509 & 1766 & 105 & 36 & 51674.0 & 51677 & -3.0 & & 53.2 & 1.8 & -195.1 \\
\hline 36 & 196 & 155523 & 1768 & 105 & 35 & $51 !$ & 51551.6 & -3.0 & 5.6 & 3.8 & 1.8 & -250.8 \\
\hline 36 & 196 & 155534 & 1770 & 105 & 34 & 51 & 528 & -3.0 & & 4.8 & 1.8 & -112.1 \\
\hline 36 & 196 & 155545 & 1772 & 105 & 33 & 51 & 59.3 & -3.1 & & 6.5 & 1.7 & -45.9 \\
\hline 36 & 196 & & 1774 & 105 & 32 & & 7.1 & -3.1 & & & 1.7 & 8.7 \\
\hline 36 & 196 & 155608 & 1776 & 105 & 31 & 51 & 09.3 & -3.1 & & & 1.7 & -22.0 \\
\hline 36 & 196 & 155618 & 1778 & 105 & 30 & & 32.1 & -3.1 & & 32.5 & 1.7 & 8.5 \\
\hline 36 & 196 & 155630 & 1780 & 105 & 29 & 51647.6 & 51650.8 & -3.2 & & 57.1 & 1.7 & 18.4 \\
\hline 36 & 196 & 155641 & 1782 & 105 & 28 & 51658.0 & 51661.2 & -3.2 & 5.4 & 63.7 & 1.7 & 12.1 \\
\hline 36 & 196 & 155651 & 1784 & 105 & 27 & 51663.8 & 51667 & -3.2 & .4 & 68.7 & 1.7 & 10.8 \\
\hline 36 & 196 & 155702 & 1786 & 105 & 26 & 51669.0 & 51672.2 & -3.2 & & 1.9 & 1.7 & 7.5 \\
\hline 36 & 196 & 155713 & 1788 & 105 & 25 & 516 & 51676.8 & -3.2 & & 8.3 & 1.7 & 10.5 \\
\hline 36 & 196 & 155723 & 1790 & 105 & 24 & 51679.0 & 51682.3 & -3.3 & & 92.1 & 1.7 & 24.3 \\
\hline 36 & 196 & 155735 & 1792 & 105 & 23 & 51681.4 & 51684.7 & -3.3 & & 81.5 & 1.7 & 2.9 \\
\hline 36 & 196 & 155745 & 1794 & 105 & 22 & 51683.4 & 51686.7 & -3.3 & & 51684.7 & 1.7 & 4.9 \\
\hline 36 & 196 & 155757 & 1796 & 105 & 21 & 51684.6 & 51687.9 & -3.3 & 51690.0 & 51688.3 & 1.7 & 8.9 \\
\hline 36 & 196 & 155814 & 1798 & 105 & 20 & 51686.0 & 51689.4 & -3.4 & 51683.4 & 51681.7 & 1.7 & -4.3 \\
\hline
\end{tabular}


TOP FIELD (nT)

BOTTOM FIELD (nT)

GRAD.

\begin{tabular}{|c|c|c|c|c|c|c|c|c|c|c|c|c|}
\hline & AY & & & $\boldsymbol{\lambda}$ & $Y$ & .) & (Corr.) & (Diff.) & (Un & (Corr.) & (Diff.) & (m) \\
\hline 36 & 196 & 327 & 1800 & 105 & 19 & & 51687.8 & -3.4 & 378.2 & 51676.5 & 1.7 & -10.2 \\
\hline 36 & 196 & 43 & 802 & 10 & 18 & 78.2 & 681.6 & -3.4 & 1687 & 51685.3 & 1.7 & 14.4 \\
\hline 36 & 196 & 358 & 304 & 105 & 17 & 62.6 & 666.1 & -3.5 & 51666.8 & 5.1 & 1.7 & 6.9 \\
\hline 36 & 196 & 155909 & 806 & 105 & 16 & 56.0 & 51659.5 & -3.5 & 655.0 & 1653.3 & 1.7 & -1.6 \\
\hline 36 & 196 & 155920 & 1808 & 105 & 15 & 51659.8 & 51663.3 & -3.5 & 51673.8 & 51672.1 & 1.7 & 23.0 \\
\hline 36 & 196 & 155930 & 1810 & 105 & 14 & 51667.4 & 51670.9 & -3.5 & 51687.4 & 51685.7 & 1.7 & 32.8 \\
\hline 36 & 196 & 155941 & 1812 & 105 & 13 & 51673.8 & 51677.3 & -3.5 & 80.4 & 51678.7 & 1.7 & 10.8 \\
\hline 36 & 196 & 155952 & 1814 & 105 & 12 & 51675.2 & 51678.8 & -3.6 & 51675.8 & 51674.1 & 1.7 & 1.0 \\
\hline 36 & 196 & 160004 & 816 & 105 & 11 & 51674.2 & 51677.8 & -3.6 & 7.4 & 1675.7 & 1.7 & 5.3 \\
\hline 3 & 196 & 1600 & 818 & 105 & 10 & 51675.8 & 51679.4 & -3.6 & 0.0 & 78.3 & 1.7 & 6.9 \\
\hline 36 & 196 & 160025 & 1820 & 105 & 0 & 51674.2 & 51677.8 & -3.6 & 51683.2 & 51681.5 & 1.7 & 14.8 \\
\hline 36 & 196 & 160036 & 1822 & 105 & 8 & 51672.4 & 51676.1 & -3.7 & 51680.6 & 51678.9 & 1.7 & 13.4 \\
\hline 36 & 196 & 160046 & 1824 & 105 & 7 & 51668.0 & 51671.7 & -3.7 & 1673.8 & 51672.1 & 1.7 & 9.5 \\
\hline 36 & 196 & 160058 & 1826 & 105 & 6 & 51662.0 & 51665.7 & -3.7 & 51665.2 & 51663.6 & 1.6 & 5.2 \\
\hline 36 & 196 & 160109 & 1828 & 105 & 5 & 51653.2 & 51656.9 & -3.7 & 51651.8 & 51650.2 & 1.6 & -2.3 \\
\hline 36 & 196 & 160120 & 1830 & 105 & 4 & 51639.6 & 51643.3 & -3.7 & 1632.8 & 51631.2 & 1.6 & -11.1 \\
\hline 3 & 196 & 160131 & 1832 & 105 & 3 & 51622.8 & 51626.6 & -3.8 & 7.6 & 6.0 & 1.6 & -24.9 \\
\hline 36 & 196 & 160 & 1834 & 105 & 2 & 51598.0 & 51601.8 & -3.8 & 1.8 & 0.2 & 1.6 & -43.0 \\
\hline 36 & 196 & 160153 & 1836 & 105 & 1 & 4.0 & 7.8 & -3.8 & .2 & 7.6 & 1.6 & -106.2 \\
\hline 36 & 196 & 160205 & 1838 & 105 & 0 & 77.8 & 51481.6 & -3.8 & 5.6 & 4.0 & 1.6 & -282.3 \\
\hline 1 & 203 & 123002 & 2 & 106 & 0 & 61.2 & 51478.6 & -17.4 & 51273.0 & 51286.0 & 13.0 & -308.5 \\
\hline 1 & 203 & 123023 & 4 & 108 & 0 & 6.8 & 51 & -17.5 & 51379.0 & 2.0 & -13.0 & -193.1 \\
\hline 2 & 203 & 123231 & 6 & 106 & 2 & 51593.8 & 51611.5 & -17.7 & 51562.2 & 5.1 & -12.9 & -51.8 \\
\hline 2 & 203 & 123310 & 8 & 108 & 2 & 51604.2 & 51622 & -17.8 & 51578.2 & 51591.1 & -12.9 & -42.6 \\
\hline 2 & 203 & 123327 & 10 & 110 & 2 & 51614.4 & 51632.3 & -17.9 & 51585.0 & 51597.9 & -12.9 & -48.2 \\
\hline 2 & 203 & 123346 & 12 & 112 & 2 & 51619.6 & 51637.5 & -17.9 & 51546.0 & 8.9 & -12.9 & -120.7 \\
\hline 3 & 203 & 123506 & 14 & 106 & 4 & 516 & 51 & -18.1 & .2 & 4.1 & -12.9 & -16.4 \\
\hline 3 & 203 & 123519 & 16 & 108 & 4 & 51 & 51 & -18.1 & 0.2 & 3.1 & -12.9 & -13.8 \\
\hline 3 & 203 & 123532 & 18 & 110 & 4 & 51 & 4.8 & -18.2 & .8 & 8.7 & -12.9 & -17.7 \\
\hline 3 & 203 & 123546 & 20 & 112 & 4 & 51 & 1.4 & -18.2 & .0 & 7.9 & -12.9 & -29.8 \\
\hline 3 & 203 & 123602 & 22 & 114 & 4 & 51571.2 & 89.4 & -18.2 & 51554.2 & 51567.1 & -12.9 & -27.9 \\
\hline 4 & 203 & 123704 & 24 & 106 & 6 & 1.2 & 51679.6 & -18.4 & 51654.6 & 51667.5 & -12.9 & -10.8 \\
\hline 4 & 203 & 123717 & 26 & 108 & 6 & 51656.6 & 51675 & -18.4 & 51652.4 & 51665.3 & -12.9 & -6.9 \\
\hline 4 & 203 & 123734 & 28 & 110 & 6 & 51649.0 & 51667.4 & -18.4 & 51644.0 & 51656.9 & -12.9 & -8.2 \\
\hline 5 & 203 & 123815 & 30 & 106 & 8 & 51669.8 & 51688.3 & -18.5 & 51665.8 & 8.7 & -12.9 & -6.6 \\
\hline 6 & 203 & 123920 & 34 & 106 & 10 & 516 & 51690.3 & -18.5 & 51 & 2.9 & -12.9 & -3.0 \\
\hline 7 & 203 & 123939 & 36 & 106 & 12 & 51 & & -18.5 & & 2.9 & -12.9 & -19.0 \\
\hline 7 & 203 & 123954 & 38 & 108 & 12 & 51 & .4 & -18.4 & .4 & 5.3 & -12.9 & -9.2 \\
\hline 8 & 203 & 124043 & 40 & 106 & 14 & 51 & 4.7 & -18.3 & & 4.5 & -12.9 & 8.5 \\
\hline 9 & 203 & 124108 & 42 & 106 & 16 & 51654.0 & 51672.3 & -18.3 & 51637.8 & 51650.6 & -12.8 & -26.6 \\
\hline 10 & 203 & 124127 & 44 & 106 & 18 & 51672.4 & 51690.6 & -18.2 & 51655.4 & 51668.2 & -12.8 & -27.9 \\
\hline 11 & 203 & 124147 & 46 & 106 & 20 & 51684.4 & 51702.6 & -18.2 & 51677.2 & 51690.0 & -12.8 & -11.8 \\
\hline 12 & 203 & 124210 & 48 & 106 & 22 & 51682.2 & 51700.3 & -18.1 & 516 & 51691.4 & -12.8 & -5.9 \\
\hline 12 & 203 & 124225 & 50 & 108 & 22 & 51685.4 & 03.5 & -18.1 & 516 & 6.0 & -12.8 & -3.6 \\
\hline 13 & 203 & 124312 & 52 & 106 & 24 & 51678.8 & 51696.7 & -17.9 & 516 & 0.8 & -12.8 & 15.1 \\
\hline 13 & 203 & 124325 & 54 & 108 & 24 & 51674.8 & 51692.7 & -17.9 & 51674.0 & 51686.8 & -12.8 & -1.3 \\
\hline 14 & 203 & 124414 & 56 & 106 & 26 & 51669.4 & 51687.2 & -17.8 & 51667.2 & 51680.0 & -12.8 & -3.6 \\
\hline 14 & 203 & 124428 & 58 & 108 & 26 & 51667.2 & 51685 & -17.8 & 51666.2 & 51679.0 & -12.8 & -1.6 \\
\hline 14 & 203 & 124440 & 60 & 110 & 26 & 51660.2 & 51677.9 & -17.7 & 51657.6 & 51670.4 & -12.8 & -4.3 \\
\hline
\end{tabular}


TOP FIELD (nT)

LINE DAY TIME STA. $X \quad Y$ (Uncorr.) (Corr.) $14 \quad 203 \quad 124453$

$\begin{array}{lll}15 & 203 & 124531\end{array}$

$\begin{array}{lll}15 & 203 & 124545\end{array}$

$\begin{array}{lll}15 & 203 & 124559\end{array}$

$\begin{array}{lll}15 & 203 & 124612\end{array}$

$\begin{array}{lll}16 & 203 & 124728\end{array}$

$\begin{array}{lll}16 & 203 & 124742\end{array}$

$\begin{array}{lll}16 & 203 & 124757\end{array}$

$\begin{array}{lll}16 & 203 & 124819\end{array}$

$\begin{array}{lll}17 & 203 & 124907\end{array}$

$\begin{array}{lll}17 & 203 & 124921\end{array}$

$\begin{array}{lll}17 & 203 & 124935\end{array}$

$\begin{array}{lll}18 & 203 & 125022\end{array}$

$\begin{array}{lll}18 & 203 & 125037\end{array}$

$\begin{array}{lll}18 & 203 & 125052\end{array}$

$19 \quad 203 \quad 125155$

$\begin{array}{lll}19 & 203 & 125213\end{array}$

$19 \quad 203 \quad 125236$

$20 \quad 203 \quad 125325$

$20 \quad 203 \quad 125340$

$20 \quad 203 \quad 125355$

$21 \quad 203 \quad 125519$

$21 \quad 203 \quad 125535$

$\begin{array}{lll}22 & 203 & 125624\end{array}$

22

23

23

23

24

24

24

24

25

25

25

25

26

26

26

26

26

27

27

27

27

27

28

28

28
6211226

$\begin{array}{lll}64 & 10628\end{array}$

$\begin{array}{lll}66 & 10828\end{array}$

$\begin{array}{lll}68 & 110 & 28\end{array}$

$\begin{array}{lll}70 & 112 & 28\end{array}$

$\begin{array}{lll}74 & 106 & 30\end{array}$

$\begin{array}{lll}76 & 108 \quad 30\end{array}$

$\begin{array}{llll}78 & 110 & 30\end{array}$

$\begin{array}{lll}80 & 112 & 30\end{array}$

$82 \quad 10632$

$\begin{array}{lll}84 & 108 & 32\end{array}$

$\begin{array}{lll}86 & 11032\end{array}$

$\begin{array}{llll}88 & 106 & 34\end{array}$

$90 \quad 108 \quad 34$

$92 \quad 11034$

$94 \quad 10636$

$96 \quad 10836$

$\begin{array}{lll}98 & 110 & 36\end{array}$

$\begin{array}{lll}100 & 106 & 38\end{array}$

$102 \quad 10838$

$\begin{array}{lll}104 & 11038\end{array}$

$108 \quad 10640$

$\begin{array}{lll}110 & 108 & 40\end{array}$

$\begin{array}{lll}112 & 106 \quad 42\end{array}$

$\begin{array}{lll}114 & 108 & 42\end{array}$

$\begin{array}{lll}116 & 10644\end{array}$

$\begin{array}{lll}118 & 10844\end{array}$

$\begin{array}{lll}120 & 110 & 44\end{array}$

$124 \quad 10646$

$\begin{array}{lll}126 & 10846\end{array}$

$\begin{array}{lll}128 & 110 & 46\end{array}$

$\begin{array}{lll}130 & 112 & 46\end{array}$

13210648

$\begin{array}{lll}134 & 10848\end{array}$

$\begin{array}{lll}136 & 110 & 48\end{array}$

$\begin{array}{lll}138 & 112 & 48\end{array}$

$\begin{array}{lll}140 & 10650\end{array}$

14210850

$144 \quad 11050$

14611250

$148 \quad 11450$

$150 \quad 10652$

$152 \quad 10852$

$\begin{array}{lll}154 & 110 & 52\end{array}$

$\begin{array}{lll}156 & 11252\end{array}$

$\begin{array}{lll}158 & 114 & 52\end{array}$

$160 \quad 10654$

162

164

11054
51643.6

51658.0

51656.6

51648.8

51634.2

51631.8

51632.6

51632.0

51631.6

51586.0

51593.8

51606.6

51528.8

51534.6

51598.6

51657.8

51828.2

51724.4

51797.8

52220.8

51820.8

51851.2

51975.6

51802.4

51853.2

51983.8

51957.2

51846.0

52145.8

51966.0

51873.4

51708.6

52001.6

51860.2

51749.0

51840.2

51790.6

51922.8

51753.6

51638.2

51703.8

52182.0

52044.6

51745.8

51655.4

51903.8

52158.0
51950.0
$51661.3-17.7$

$51675.6-17.6$

$51674.2-17.6$

$51666.3-17.5$

$51651.7-17.5$

$51649.4-17.6$

$51650.2-17.6$

$51649.6-17.6$

$51649.3-17.7$

$51603.9-17.9$

$51611.7-17.9$

$51624.6-18.0$

$51546.9-18.1$

$51552.8-18.2$

$51616.8-18.2$

$51676.2-18.4$

$51846.7-18.5$

$51743 \quad-18.6$

$51816.5-18.7$

$52239.6-18.8$

$51839.6-18.8$

$51870 \quad-18.8$

$51994.6-19.0$

$51821.4-19.0$

$51872.2-19.0$

$52002.7-18.9$

$51976.1-18.9$

$51864.9-18.9$

$52164.8-19.0$

$51984.7-18.7$

$51892.1-18.7$

$51727.4-18.8$

$52020.5-18.9$

$51879.1-18.9$

$51968.9-18.9$

$51768-19.0$

$51859.3-19.1$

$51809.7-19.1$

$51941.9-19.1$

$51772.8-19.2$

$51657.4-19.2$

$51723.1-19.3$

$52201.3-19.3$

$52063.9-19.3$

$51765.2-19.4$

$51674.8-19.4$

$51923.3-19.5$

$52177.5-19.5$

$51980.2-19.6$
BOTTOM FIELD (nT)

(Uncorr.) (Corr.) (Diff.)

$\begin{array}{lll}51639.0 & 51651.7 & -12.7\end{array}$

$51658.8 \quad 51671.5 \quad-12.7$

$51657.0 \quad 51669.7 \quad-12.7$

$51648.2 \quad 51660.9 \quad-12.7$

$51633.2 \quad 51645.9 \quad-12.7$

$51629.6 \quad 51642.3 \quad-12.7$

51627.6

51633.0

51640.3

51620.2

$51645.7 \quad-12.7$

$51632.9-12.7$

51568.0

$51580.8-12.8$

51560.2

$51573.0-12.8$

51594.0

51456.4

51447.6

51536.6

51558.2

51466.8

51620.6

51678.0

52685.0

51765.0

51877.0

52099.0

51714.4

51841.4

52077.8

52098.4

51862.6

52355.6

51979.8

51942.6

51665.6

52122.4

51718.2

52163.4

51713.4

51933.4

51594.0

51936.4

51716.0

51618.2

51485.8

52763.8

52053.2

51692.6

51627.4

51717.6

52294.4

51908.8

$51606.8-12.8$

$51469.2-12.8$

$51460.4-12.8$

$51549.4 \quad-12.8$

$51571.0 \quad-12.8$

$51479.6-12.8$

$51633.4 \quad-12.8$

$51690.9-12.9$

$52697.9-12.9$

$51777.9-12.9$

$51889.9-12.9$

$52111.9-12.9$

$51727.4-13.0$

$51854.4-13.0$

$52090.8-13.0$

$52111.4-13.0$

$51875.6-13.0$

$52368.7-13.1$

$51993.3-13.5$

$51956.1-13.5$

$51679.1-13.5$

$52136.0-13.6$

$51731.8-13.6$

$52177.0-13.6$

$51727.0-13.6$

$51947.0-13.6$

$51607.6-13.6$

$51950.0-13.6$

$51729.6-13.6$

$51631.8-13.6$

$\begin{array}{lll}51499.5 & -13.7\end{array}$

$52777.5-13.7$

$52066.9-13.7$ 
TOP FIELD (nT)

$\begin{array}{cccccccc}\text { LINE DAY } & \text { TIME } & \text { STA. } & X & Y & \text { (Uncorr.) } & \text { (Corr.) } \\ 28 & 203 & 132334 & 166 & 112 & 54 & 51756.0 & 51775.6\end{array}$

$\begin{array}{lllllll}28 & 203 & 132357 & 168 & 114 & 54 & 516\end{array}$

$\begin{array}{llllll}29 & 203 & 132455 & 170 & 106 & 56\end{array}$

$\begin{array}{llllll}29 & 203 & 132508 & 172 & 108 & 56\end{array}$

$\begin{array}{lllllll}29 & 203 & 132522 & 174 & 110 & 56\end{array}$

$\begin{array}{llllll}29 & 203 & 132534 & 176 & 112 & 56\end{array}$

$\begin{array}{lllllll}29 & 203 & 132546 & 178 & 114 & 56\end{array}$

$\begin{array}{llllll}30 & 203 & 132637 & 180 & 106 & 58\end{array}$

$\begin{array}{llllll}30 & 203 & 132651 & 182 & 108 & 58\end{array}$

$\begin{array}{llllll}30 & 203 & 132706 & 184 & 110 & 58\end{array}$

$\begin{array}{llllll}30 & 203 & 132719 & 186 & 112 & 58\end{array}$

$\begin{array}{llllll}30 & 203 & 132731 & 188 & 114 & 58\end{array}$

$\begin{array}{llllll}31 & 203 & 132933 & 192 & 106 & 60\end{array}$

$\begin{array}{llllll}31 & 203 & 132946 & 194 & 108 & 60\end{array}$

$\begin{array}{llllll}31 & 203 & 132959 & 196 & 110 & 60\end{array}$

$\begin{array}{llllll}31 & 203 & 133013 & 198 & 112 & 60\end{array}$

31

32

32

32

32

33

33

33

34

34

34

34

34

34

34

35

35

35

35

35

35

36

36

36

36

36

36

36

37

37

37

37

37
203133026200

$203 \quad 133115 \quad 202$

$203 \quad 133128 \quad 204$

$\begin{array}{lll}203 & 133141 \quad 206\end{array}$

$\begin{array}{lll}203 & 133155 \quad 208\end{array}$

$\begin{array}{lll}203 & 133245 \quad 210\end{array}$

$\begin{array}{lll}203 & 133259 & 212\end{array}$

$\begin{array}{lll}203 & 133312 & 214\end{array}$

$\begin{array}{lll}203 & 133447 \quad 216\end{array}$

$\begin{array}{lll}203 & 133459 & 218\end{array}$

$\begin{array}{lll}203 & 133512 & 220\end{array}$

$\begin{array}{lll}203 & 133525 \quad 222\end{array}$

$\begin{array}{lll}203 & 133538 & 224\end{array}$

$\begin{array}{lll}203 & 133551 \quad 226\end{array}$

$\begin{array}{lll}203 & 133606 \quad 228\end{array}$

$\begin{array}{lll}203 & 133825 & 230\end{array}$

$\begin{array}{lll}203 & 133838 \quad 232\end{array}$

$\begin{array}{lll}203 & 133851 \quad 234\end{array}$

$203 \quad 133903 \quad 236$

$\begin{array}{lll}203 & 133916 \quad 238\end{array}$

$203 \quad 133929 \quad 240$

$203 \quad 134253 \quad 246$

$\begin{array}{llll}203 & 134309 & 248\end{array}$

$\begin{array}{lll}203 & 134327 & 250\end{array}$

$\begin{array}{llll}203 & 134343 \quad 252\end{array}$

$\begin{array}{lll}203 & 134357 & 254\end{array}$

$\begin{array}{lll}203 & 134412 \quad 256\end{array}$

$\begin{array}{lll}203 & 134439 & 258\end{array}$

$\begin{array}{lll}203 & 134617 \quad 260\end{array}$

$\begin{array}{lll}203 & 134634 & 262\end{array}$

$203134648 \quad 264$

$203 \quad 134702 \quad 266$

268
11460

10662

10862

11062

11262

10664

10864

11064

10666

10866

11066

11266

11466

11666

11866

10668

10868

11068

11268

11468

11668

10670

10870

11070

11270

11470

11670

11870

10672

10872

11072

11272

11472
51680.2

52441.0

52114.2

51923.0

51768.2

51687.0

52809.6

52231.2

51913.0

51766.8

51689.6

52263.2

52097.4

51853.6

51756.6

51685.0

51963.2

51888.4

51801.4

51742.8

51850.2

51810.8

51770.6

51792.6

51771.2

51741.8

51709.8

51654.4

51465.6

51369.4

51750.4

51736.0

51717.2

51693.6

51636.2

51449.2

51686.8

51683.4

51672.0

51652.2

51604.6

51470.8

51292.2

51549.2

51517.6

51545.6

51550.6

51509.8
$51775.6-19.6$

$51699.8-19.6$

$52460.8-19.8$

$52134-19.8$

$51942.8-19.8$

$51788-19.8$

$51706.9-19.9$

$52829.6-20.0$

$52251.2-20.0$

$51933-20.0$

$51786.8-20.0$

$51709.7-20.1$

$52283.3-20.1$

$52117.6-20.2$

$51873.8-20.2$

$51776.8-20.2$

$51705.2-20.2$

$51983.4-20.2$

$51908.6-20.2$

$51821.6-20.2$

$\begin{array}{lll}51763 & -20.2\end{array}$

$51870.3-20.1$

$51830.9-20.1$

$51790.7-20.1$

$51812.7-20.1$

$51791.3-20.1$

$51761.9-20.1$

$51729.9-20.1$

$51674.5-20.1$

$51485.7-20.1$

$51389.5-20.1$

$51770.5-20.1$

$51756-20.0$

$51737.2-20.0$

$51713.6-20.0$

$51656.2-20.0$

$51469.2-20.0$

$51706.7-19.9$

$51703.2-19.8$

$51691.8-19.8$

$\begin{array}{lll}51672 & -19.8\end{array}$

$51624.3-19.7$

$51490.5-19.7$

$51311.9-19.7$

$51568.7-19.5$

$51537-19.4$

$51565-19.4$

$51570-19.4$

$51529.1-19.3$
BOTTOM FIELD (nT)

GRAD.

(Uncorr.) (Corr.) (Diff.)

(nT/m)

$\begin{array}{llll}51711.2 & 51724.9 & -13.7 & -73.4\end{array}$

$\begin{array}{llll}51650.4 & 51664.2 & -13.8 & -48.8\end{array}$

$\begin{array}{llll}52569.6 & 52583.4 & -13.8 & 210.8\end{array}$

$52013.8 \quad 52027.6 \quad-13.8 \quad-164.6$

$\begin{array}{llll}51901.8 & 51915.6 & -13.8 & -34.8\end{array}$

$\begin{array}{lllll}51734.0 & 51747.8 & -13.8 & -56.1\end{array}$

$\begin{array}{llll}51669.8 & 51683.6 & -13.8 & -28.2\end{array}$

$\begin{array}{llll}53453.6 & 53467.4 & -13.8 & 1055.7\end{array}$

$\begin{array}{llll}52287.0 & 52300.8 & -13.8 & 91.5\end{array}$

$\begin{array}{llll}51872.6 & 51886.5 & -13.9 & -66.2\end{array}$

$\begin{array}{llll}51737.0 & 51750.9 & -13.9 & -48.9\end{array}$

$\begin{array}{llll}51674.6 & 51688.5 & -13.9 & -24.6\end{array}$

$\begin{array}{lllll}52175.8 & 52189.7 & -13.9 & -143.3\end{array}$

$\begin{array}{llll}52156.6 & 52170.5 & -13.9 & 97.0\end{array}$

$\begin{array}{lllll}51806.8 & 51820.7 & -13.9 & -76.7\end{array}$

$\begin{array}{llll}51736.0 & 51749.9 & -13.9 & -33.8\end{array}$

$51672.0 \quad 51685.9 \quad-13.9 \quad-21.3$

$\begin{array}{llll}51905.2 & 51919.1 & -13.9 & -95.1\end{array}$

$\begin{array}{llll}51844.6 & 51858.5 & -13.9 & -71.8\end{array}$

$\begin{array}{llll}51774.8 & 51788.7 & -13.9 & -43.6\end{array}$

$\begin{array}{llll}51723.0 & 51736.9 & -13.9 & -32.5\end{array}$

$\begin{array}{lllll}51818.2 & 51832.0 & -13.8 & -52.5\end{array}$

$\begin{array}{llll}51786.6 & 51800.4 & -13.8 & -39.7\end{array}$

$51754.2 \quad 51768.0 \quad-13.8 \quad-26.9$

$51775.2 \quad 51789.0 \quad-13.8 \quad-28.5$

51756.2

51738.0

51703.8

51647.4

51441.8

50558.2

51752.0

51731.6

51713.2

51681.0

51634.2

51400.6

51682.6

51680.6

51674.8

51661.6

51605.8

51422.8

50233.0

51554.0

51547.6

51568.6

51547.8

51497.0

$51770.0 \quad-13.8$

$51751.8 \quad-13.8$

$\begin{array}{ll}51717.6 & -13.8\end{array}$

$51661.2 \quad-13.8$

$51455.6-13.8$

$50572.0-13.8$

$\begin{array}{ll}51765.8 & -13.8\end{array}$

$\begin{array}{lll}51745.3 & -13.7\end{array}$

$\begin{array}{lll}51726.9 & -13.7\end{array}$

$51694.7 \quad-13.7$

$51647.9-13.7$

$51414.3 \quad-13.7$

$51696.2-13.6$

$51694.1-13.5$

$51688.3-13.5$

$51675.1-13.5$

$51619.2-13.4$

$51436.2-13.4$ 
TOP FIELD (nT)

LINE DAY TIME STA. $X Y$ (Uncorr.) (Corr.)

$\begin{array}{lll}37 \quad 203 & 134729\end{array}$

$\begin{array}{lll}37 & 203 & 134742\end{array}$

$\begin{array}{lll}38 & 203 \quad 135146\end{array}$

$\begin{array}{lll}38 & 203 \quad 135203\end{array}$

38

38

38

38

38

38

38

39

39

39

39

39

39

39

39

39

39

40

40

40

40
40

40

40

40

40

41

41

41

41

41

41

41

41

41
203135217

203135233

203135250

203135307

203135358

$203 \quad 135412$

203135500

203135756

203135815

203135830

203135905

203135918

203135931

203135945

203140000

203140014

203140112

203140535

203140547

203140607

203140620

203140637

203140649

203140705

203140719

203140734

203140859

203140911

203140923

203140936

203140952

203141008

$\begin{array}{lll}203 & 141029 & 344\end{array}$

$203 \quad 141114 \quad 348$

$203 \quad 141132 \quad 350$
1167251362.8

$\begin{array}{lll}118 & 52 & 50835.0\end{array}$

$10674 \quad 50971.6$

$\begin{array}{lll}108 & 74 & 49676.2\end{array}$

$\begin{array}{lll}11074 & 51129.6\end{array}$

$11274 \quad 51209.8$

11474

50997.0

51019.0

50941.0

51517.8

51657.4

51445.4

51337.6

51400.0

51409.0

51410.4

51328.2

51601.4

52304.4

51618.8

51637.4

51642.6

51630.8

51604.2

51584.2

51550.2

51497.2

51906.2

51880.6

51639.6

51698.2

51691.2

51682.4

51681.4

51670.8

51669.6

51637.6

51707.8

51705.4
$513821-19.3$

$50854.3-19.3$

$50990.4 \quad-18.8$

$\begin{array}{lll}49695 & -18.8\end{array}$

$51148.3-18.7$

$51228.5-18.7$

$51015.7-18.7$

$51037.6-18.6$

$50959.5-18.5$

$51536.3-18.5$

$\begin{array}{ll}51675.8 & -18.4\end{array}$

$51463.4-18.0$

$51355.6-18.0$

$\begin{array}{ll}51418 & -18.0\end{array}$

$51426.9-17.9$

$51428.3-17.9$

$\begin{array}{ll}51346 & -17.8\end{array}$

$51619.2-17.8$

$52322.2-17.8$

$51636.6-17.8$

$\begin{array}{ll}51655 & -17.6\end{array}$

$51659.7-17.1$

$51647.9-17.1$

$51621.2-17.0$

$51601.2-17.0$

$51567.2-17.0$

$51514.1-16.9$

$51923.1-16.9$

$51897.5-16.9$

$51656.5-16.9$

$51714.9-16.7$

$51707.9-16.7$

$51699-16.6$

$51698-16.6$

$51687.4-16.6$

$51686.1-16.5$

$51654.1-16.5$

$51724.2-16.4$

$51721.8-16.4$
BOTTOM FIELD (nT)

(Uncorr.) (Corr.) (Diff.)

$\begin{array}{llll}51354.0 & 51367.0 & -13.0\end{array}$

51812.6

50854.2

50160.4

51144.4

51014.0

50869.8

51074.8

50263.8

51404.0

51638.4

51355.0

51042.6

51258.4

51215.4

51144.6

51099.0

50410.6

51925.2

50543.2

51593.8

51615.2

51597.2

51572.2

51542.8

51480.8

51226.2

51430.0

52238.0

51503.4

51692.8

51684.0

51675.4

51692.8

51654.2

51622.8

51530.0

51632.8

51684.4
$51825.6-13.0$

$50866.7-12.5$

$50172.9-12.5$

$51156.8-12.4$

$51026.4-12.4$

$50882.2-12.4$

$51087.1-12.3$

$50276.0-12.2$

$51416.2-12.2$

$51650.6-12.2$

$51366.7-11.7$

$51054.3 \quad-11.7$

$51270.1 \quad-11.7$

$51227.0-11.6$

$51156.2-11.6$

$51110.5 \quad-11.5$

$50422.1-11.5$

$51936.7-11.5$

$50554.7-11.5$

$51605.1-11.3$

$51626.0-10.8$

$51608.0-10.8$

$51582.9-10.7$

$51553.5-10.7$

$51491.5-10.7$

$51236.8-10.6$

$51440.6-10.6$

$52248.6 \quad-10.6$

$51514.0-10.6$

$51703.2 \quad-10.4$

$51694.4-10.4$

$51685.7-10.3$

$51703.1-10.3$

$51664.5-10.3$

$51633.0-10.2$

$51540.2-10.2$

$51642.9-10.1$

$51694.5-10.1$
GRAD.

(nT/m)

$-14.4$

1602.6

$-192.5$

793.8

24.3

$-321.0$

$-208.5$

91.5

$-1110.2$

$-186.6$

$-31.1$

$-148.2$

$-483.6$

$-232.1$

$-317.4$

$-435.7$

$-375.7$

$-1952.1$

$-621.6$

$-1763.3$

$-71.5$

$-44.9$

$-55.1$

$-52.5$

$-67.9$

$-113.8$

$-444.3$

$-780.7$

585.9

$-223.3$

$-8.9$

$-11.8$

$-11.5$

18.7

$-27.2$

$-76.7$

$-176.4$

$-123.0$

$-34.4$ 


\section{FIGURE CAPTIONS}

Figure 1. Location of D-Area Oil Basin Waste Unit on SRS.

Figure 2. Aerial photograph of $D$ Area Oil Basin when in use. A metal fence, the remnants of which, currently surround the waste unit shown in outline. Also shown is coordinate reference for the magnetic survey. At least one trench can be discerned approximately located along the $40-50$ meter y coordinate. In addition another, thinner linear feature can be seen parallel to and approximately 15 meters behind the trench along the 60 meter $y$ coordinate.

Figure 3. Typical temporal variations exhibited by the total magnetic field due to diurnal variation, micropulsations, and magnetic storms on the sun (adapted from Breiner, 1973).

Figure 4. Superposition of the earth's ambient magnetic field and anomalous fields produced by induced monopoles and dipoles to produce anomalies in the total field (adapted from Breiner, 1973).

Figure 5. The relationship of magnetic inclination and declination to geographic directions on the earth (adapted from Telford and others, 1976).

Figure 6. Typical profiles of magnetic field intensity across monopoles and dipoles for conditions similar to SRS. Also shown is a contour representation of magnetic field intensity over a dipole with accompanying representative profiles (adapted from Breiner, 1973).

Figure 7. Magnetic intensity profiles across several geometric shapes of interest for magnetic field inclination similar to that of SRS (i.e. 60 degrees) (adapted from Breiner, 1973).

Figure 8. The intensity of the total magnetic field compared with the vertical gradient over a prismatic body (adapted from Breiner, 1973).

Figure 9. Model total field and vertical gradient magnetic profiles for single 55 gallon steel drums in vertical position with the top at various distances below the sensor. Profiles calculated for a total field intensity of $53220 \mathrm{nT}$ and northern hemisphere inclination of 70 degrees (adapted from Gilkeson and others, 1990).

Figure 10. Contour representation of total field anomaly calculated for a single 55 gallon steel drum in vertical position with the top $10 \mathrm{ft}$ below the sensor. Calculation based on a total field intensity of $53220 \mathrm{nT}$ and northern hemisphere inclination of 70 degrees (adapted from Gilkeson and others, 1990). 
Figure 11. Contour representation of a cashe of 5 steel 55 gallon drums in a $12 \mathrm{ft}$ by $12 \mathrm{ft}$ area with the top of the drums $10 \mathrm{ft}$ below the sensor. Calculation based on a total field intensity of $53220 \mathrm{nT}$ and northern hemisphere inclination of 70 degrees (adapted from Gilkeson and others, 1990).

Figure 12. a) Planimetric map of D Area Oil Basin Waste Unit, based on the Planimetric Map of "D" Area Oil Seepage Basin prepared by Cranston, Robertson and Whitehurst, P.C., 1988. b) Planimetric information overlain onto aerial photograph of D Area Oil Basin when the trenches were open.

Figure 13. Locations of ground penetrating radar survey lines.

Figure 14. Station locations for D-Area Oil Basin Magnetic Survey. Station locations marked by "+"

Figure 15. Color contoured D-Area Oil Basin magnetic survey diurnally corrected top sensor data. Data values represent total field - $51729.4 \mathrm{nT}$.

Figure 16. Color contoured D-Area Oil Basin magnetic survey diurnally corrected bottom sensor data. Data values represent total field - $51768.2 \mathrm{nT}$.

Figure 17. Color contoured D-Area magnetic survey vertical gradient.

Figure 18. Aerial photograph of D-Area Oil Basin Waste Unit when trenches were open with vertical gradient magnetic survey data overlain. Blue contours represent positive gradient values above $100 \mathrm{nT} / \mathrm{m}$. Yellow contours represent negative gradient values below $-100 \mathrm{nT} / \mathrm{m}$. Note that linear magnetic anomalies align very well with linear features on photograph that may represent trenches.

Figure 19. Color contoured vertical gradient magnetic data of $D$-Area Oil Basin with trench boundaries and the locations of isolated magnetic anomalies shown. 


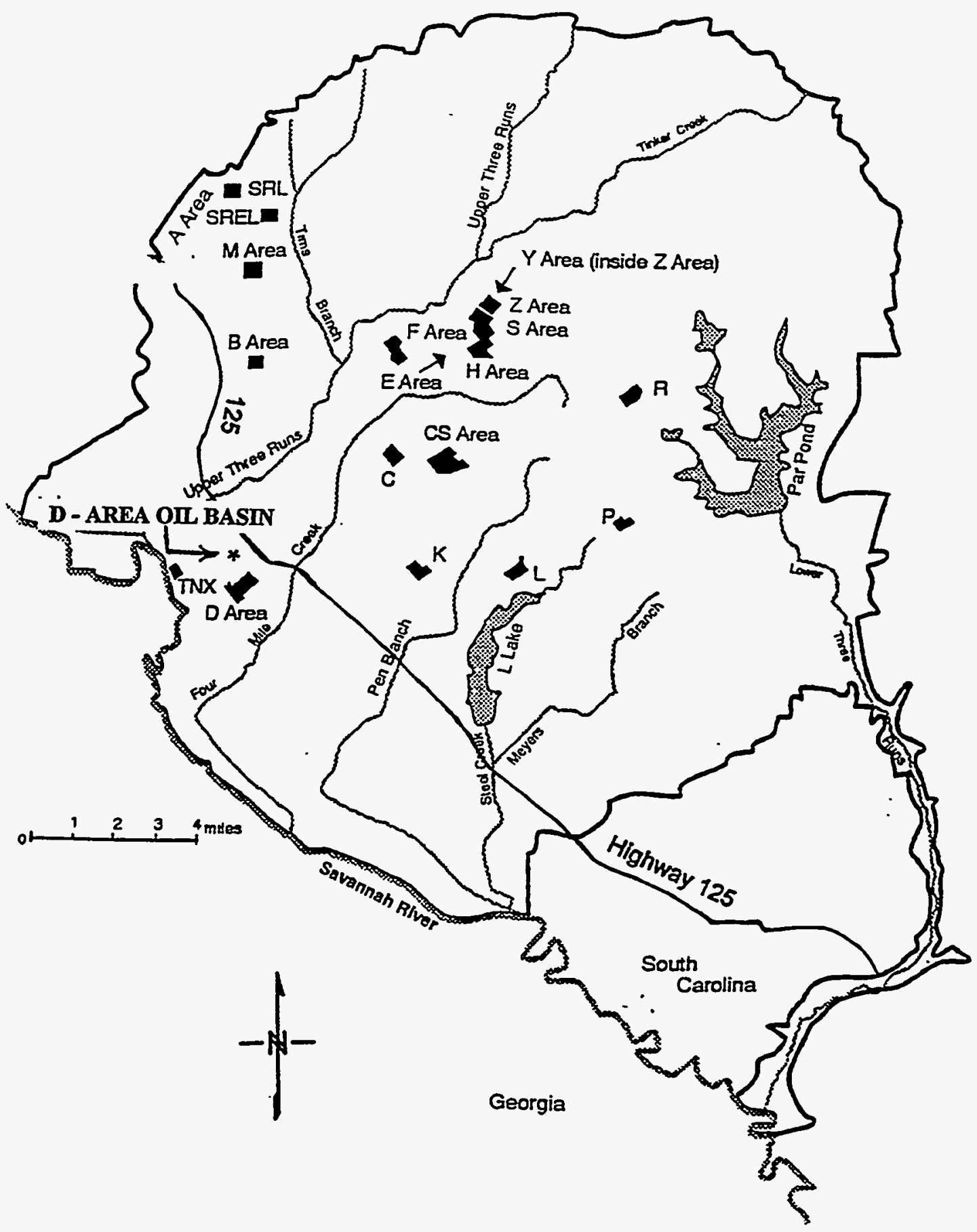




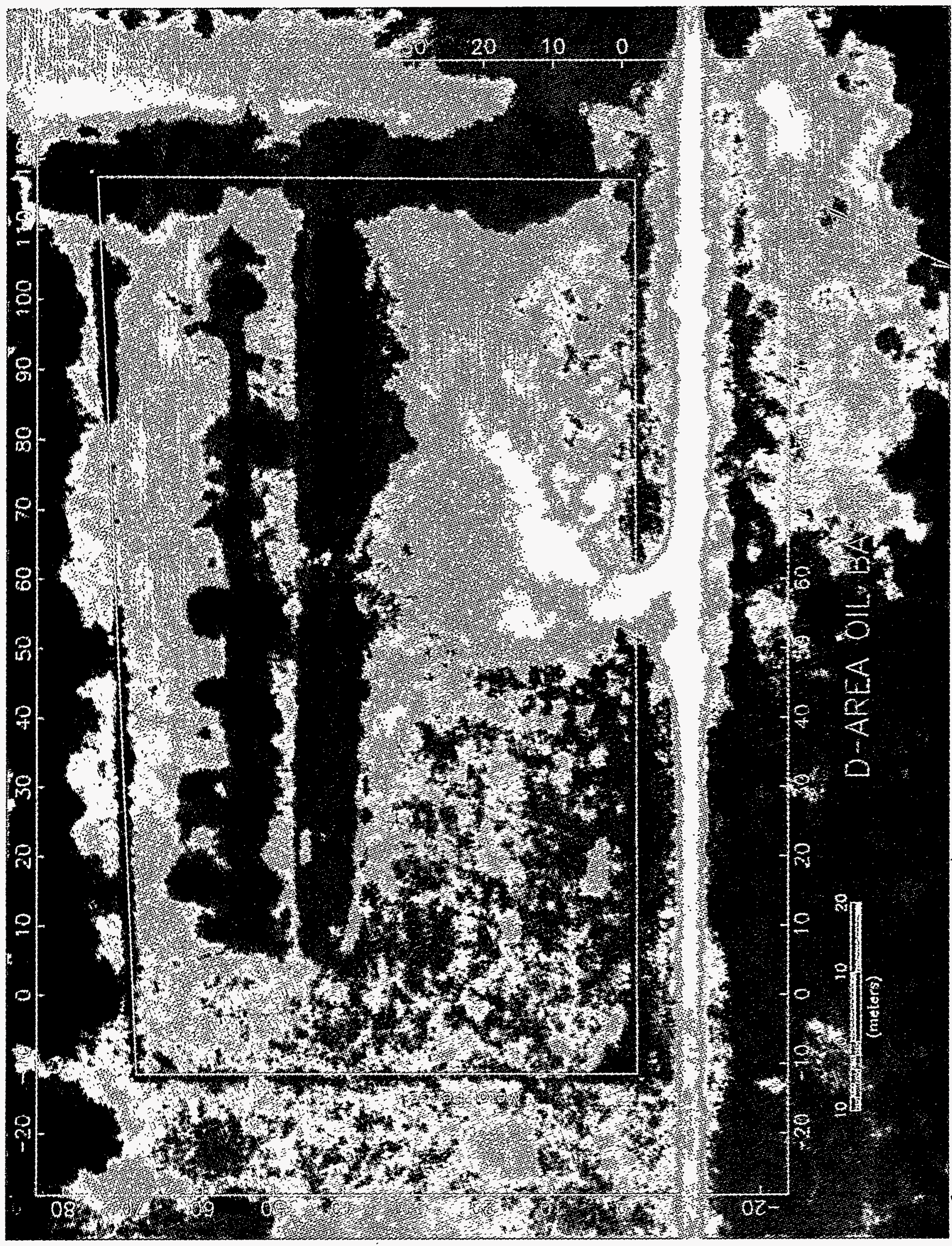




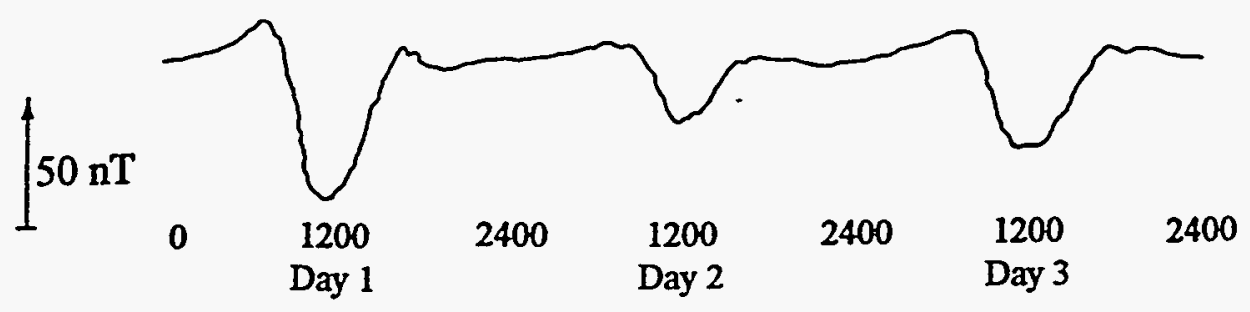

\section{DIURNAL VARIATION}

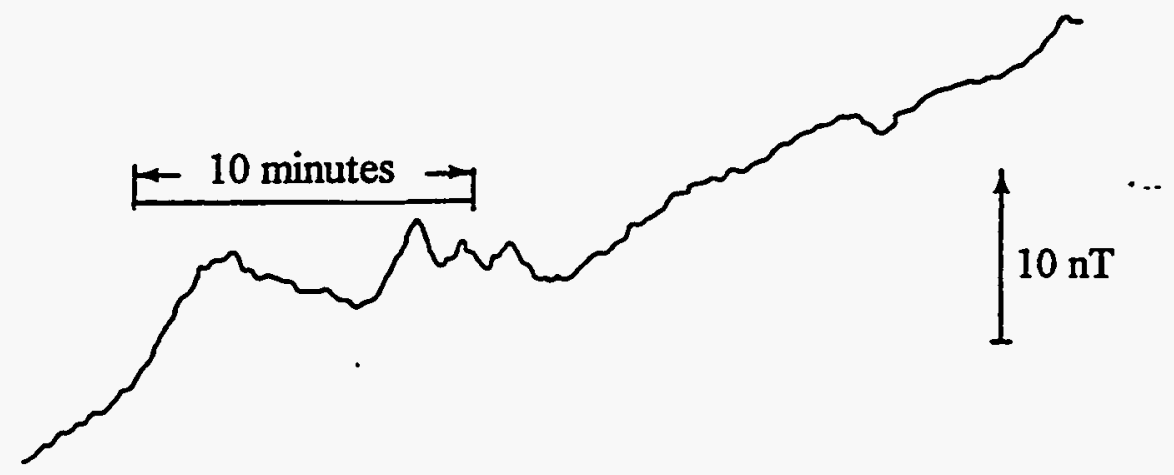

MICROPULSATIONS

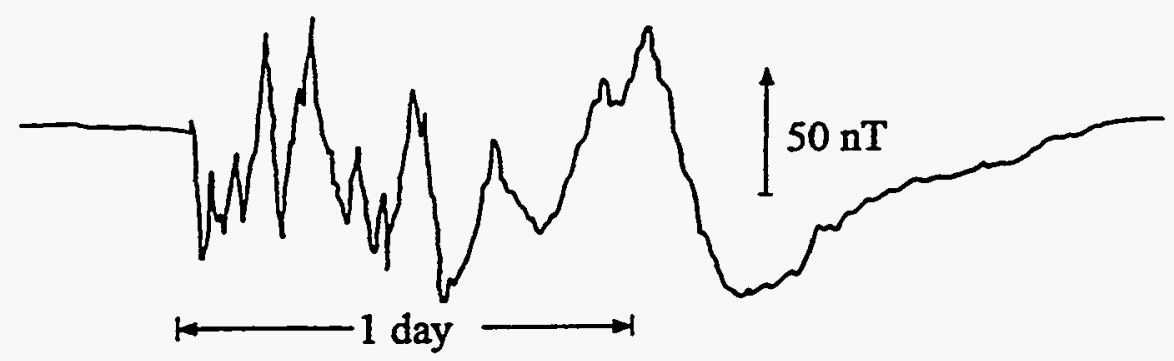

MAGNETIC STORM

Doil Bnsing Magnetic Sarwey

FIG, 3 Bure Bow Magnetic Sarvey 


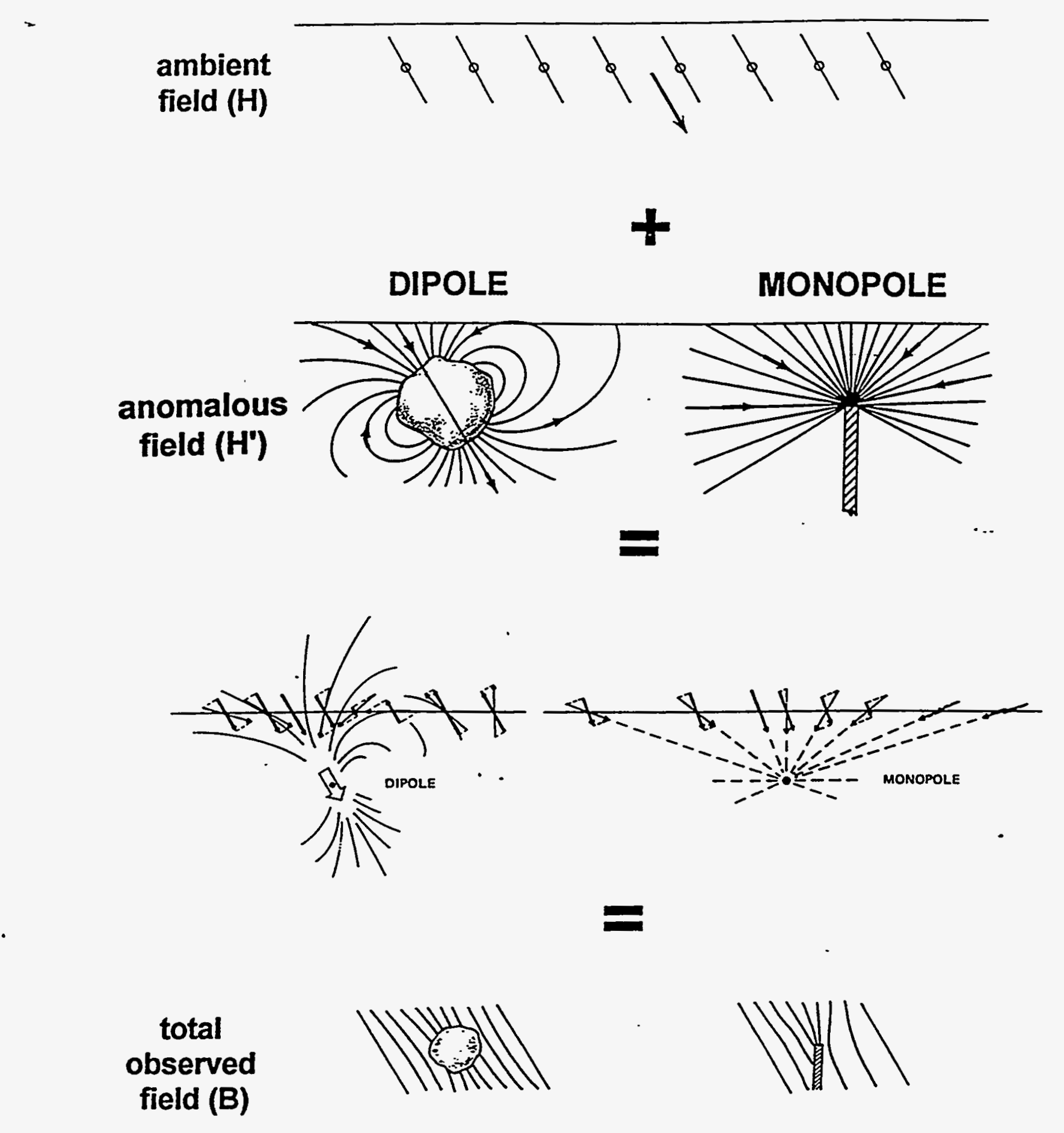




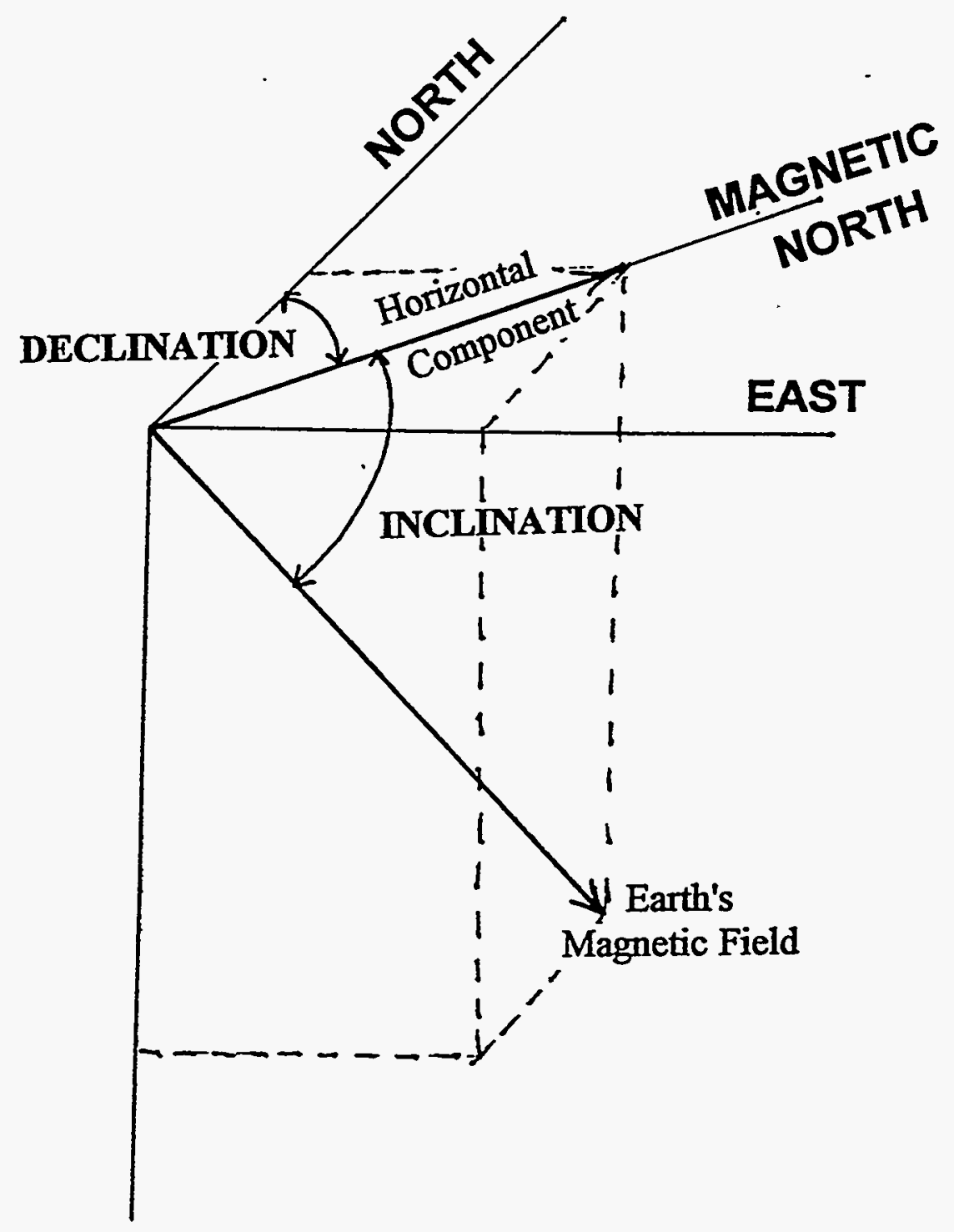

Figure 50 Acen Mogmefie Survey 

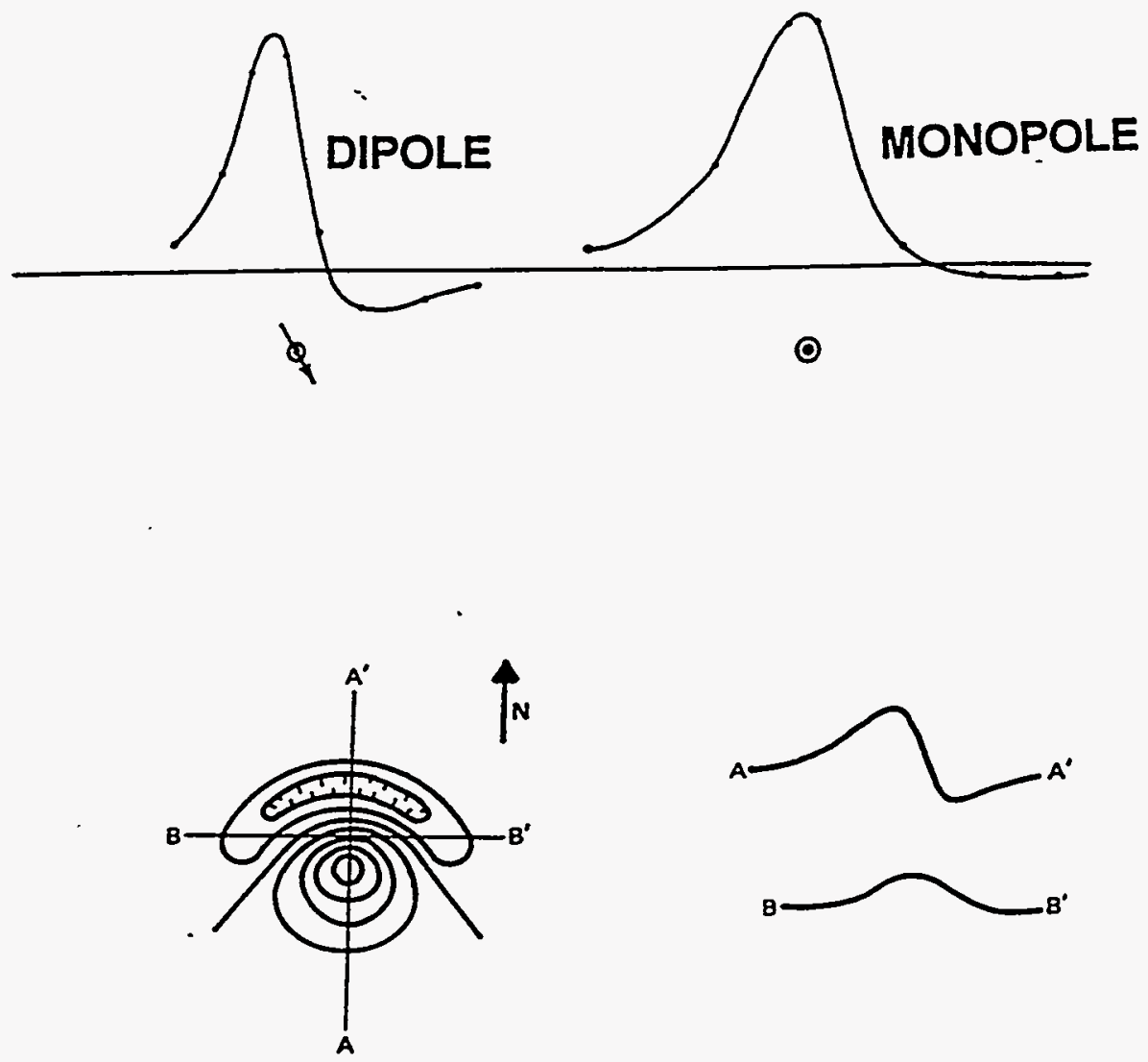

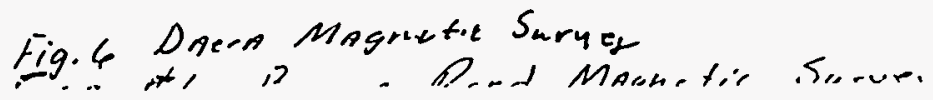




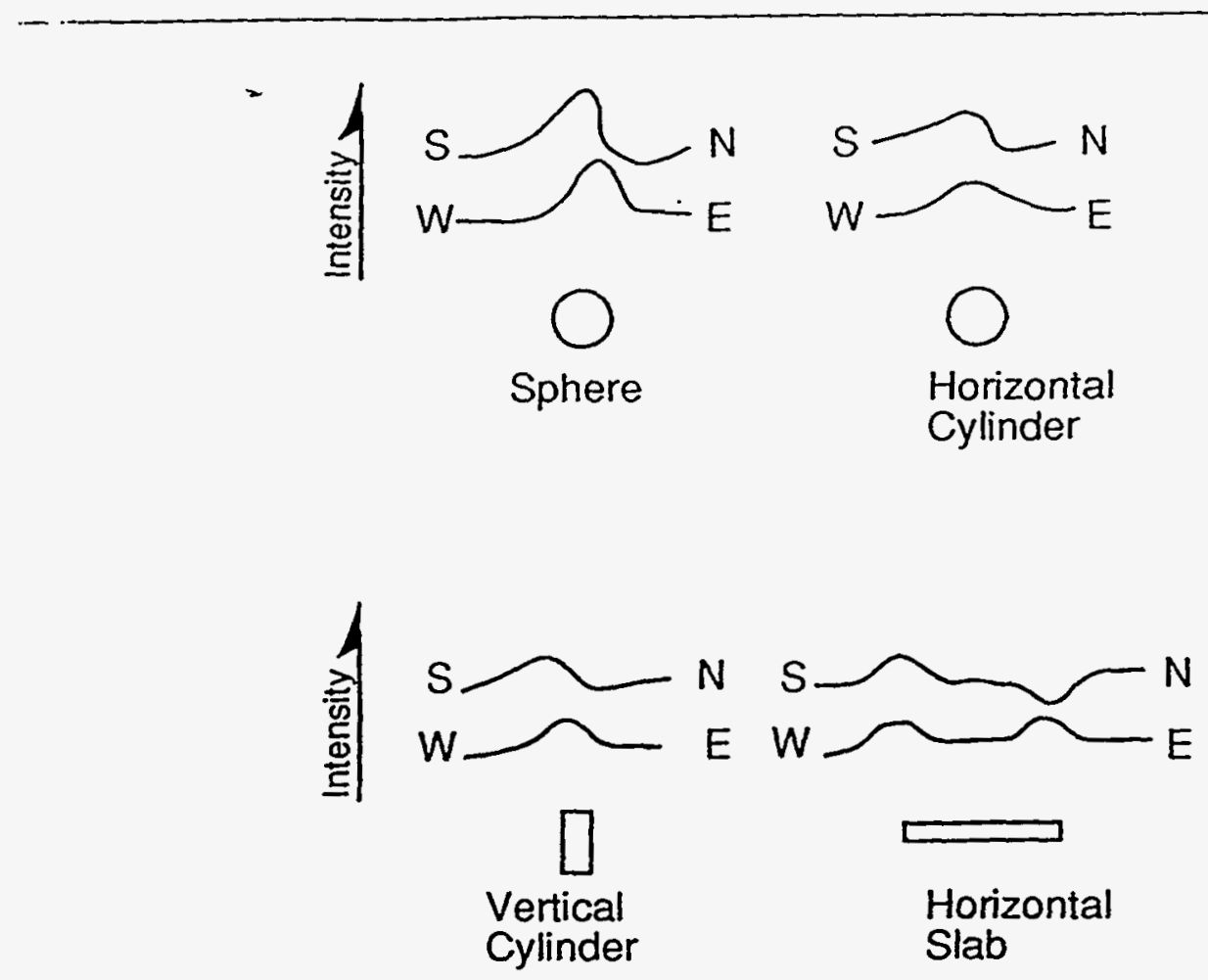

M93X2091.05.AlL

Figure 6.7 Magnetic intensity profiles across several geometric shapes of interest for magnetic field inclination similar to that of SRS (i.e., 60 degrees) (adapted from Breiner, 1973).
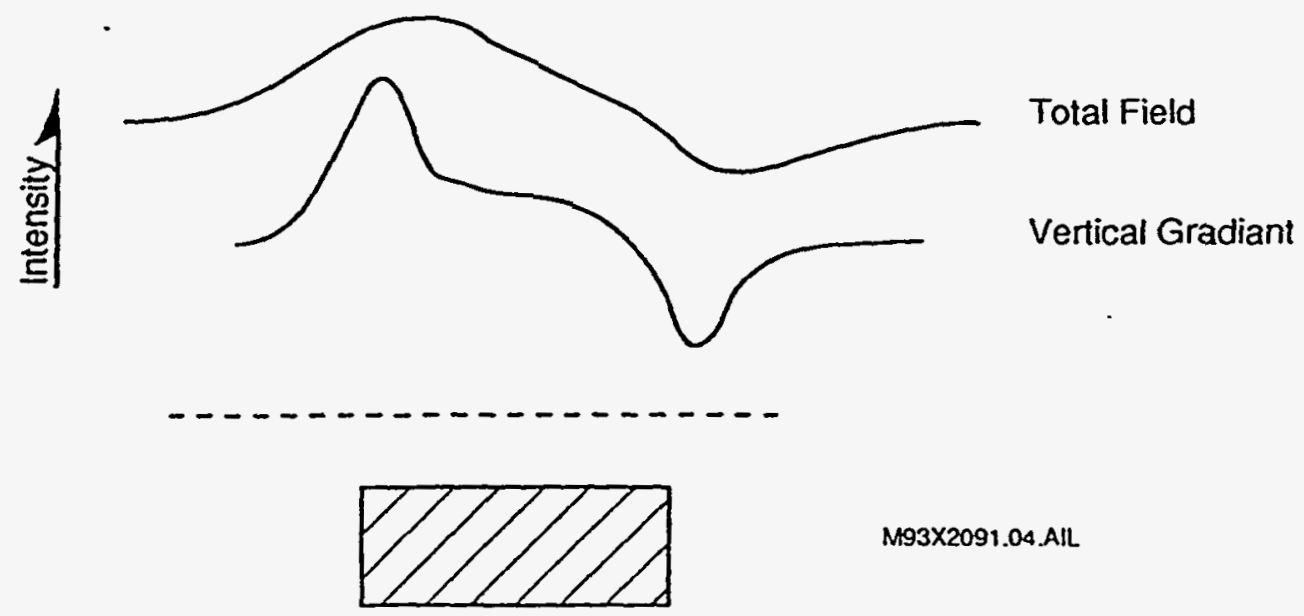

M93X2091.04.AlL

Figure $\$ 8$ The intensity of the total magnetic field compared with the verical gradient over a prismatic body (adapted from Breiner, 1973). 


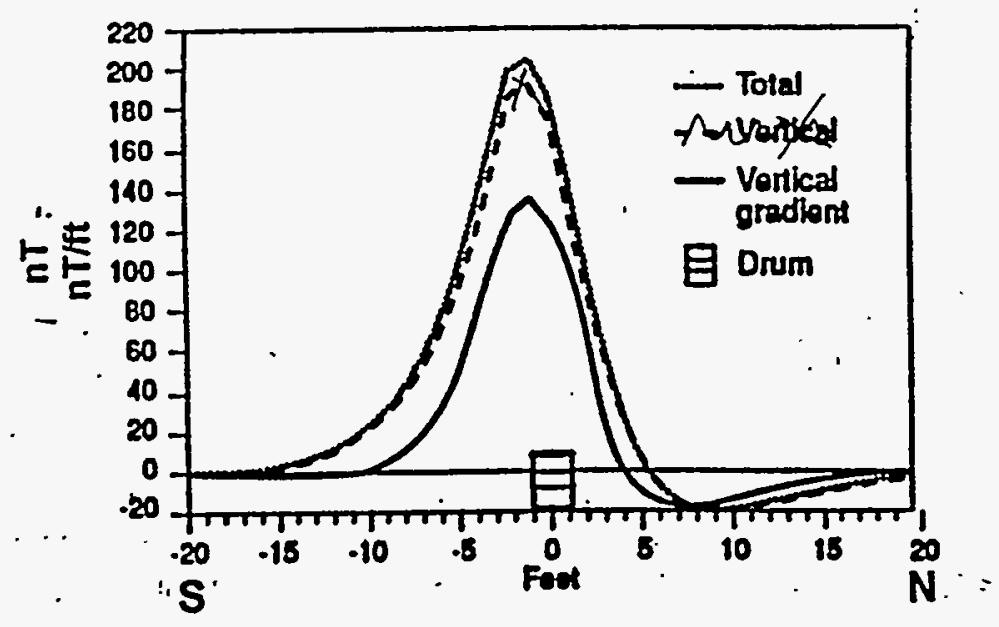

SINGLE DRUM

$(6 \mathrm{ft})$

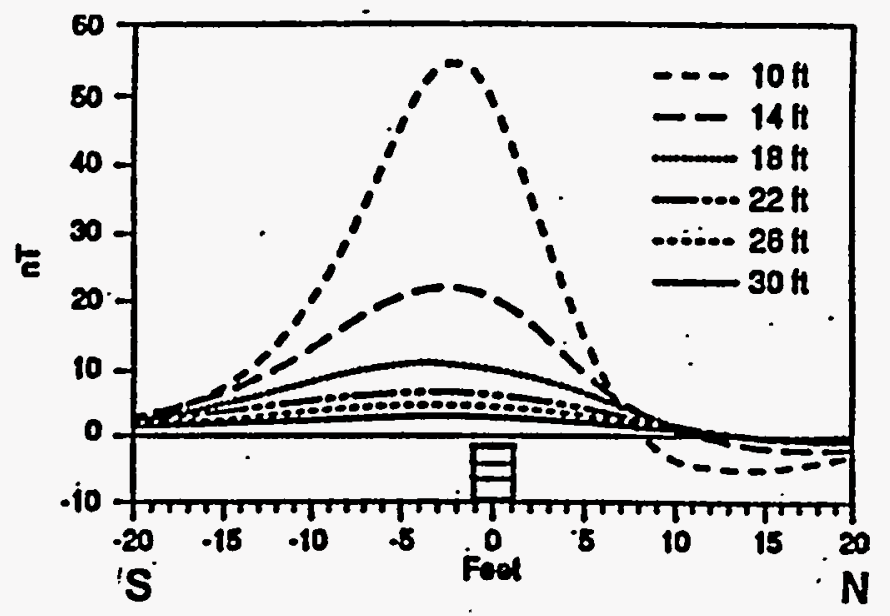

TOTAL FIELD SINGLE DRUM $(10-30 \mathrm{ft})$

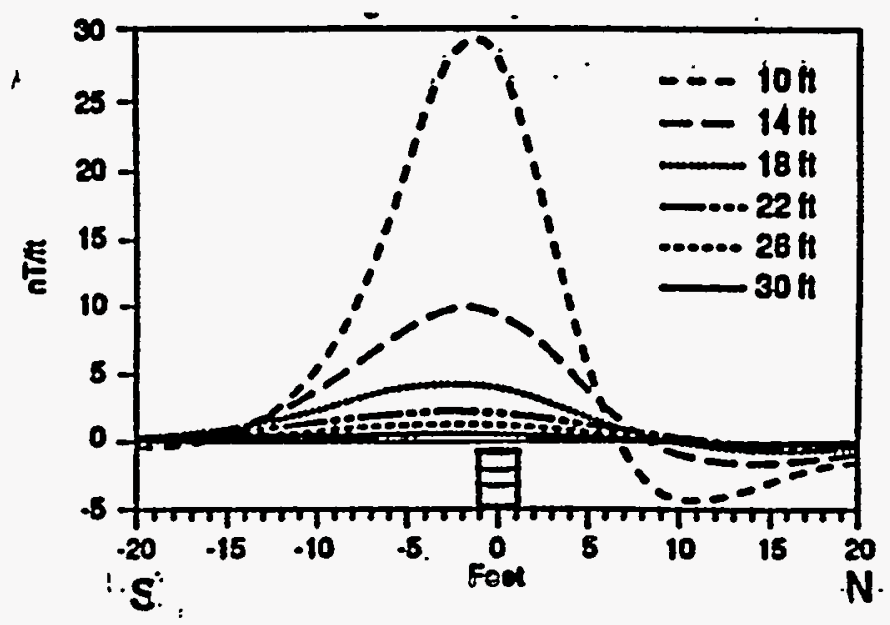

GRADIENT
SINGLE DRUM
$(10-30 \mathrm{ft})$

F76.89 Doir Boissing Mangetic Survay 


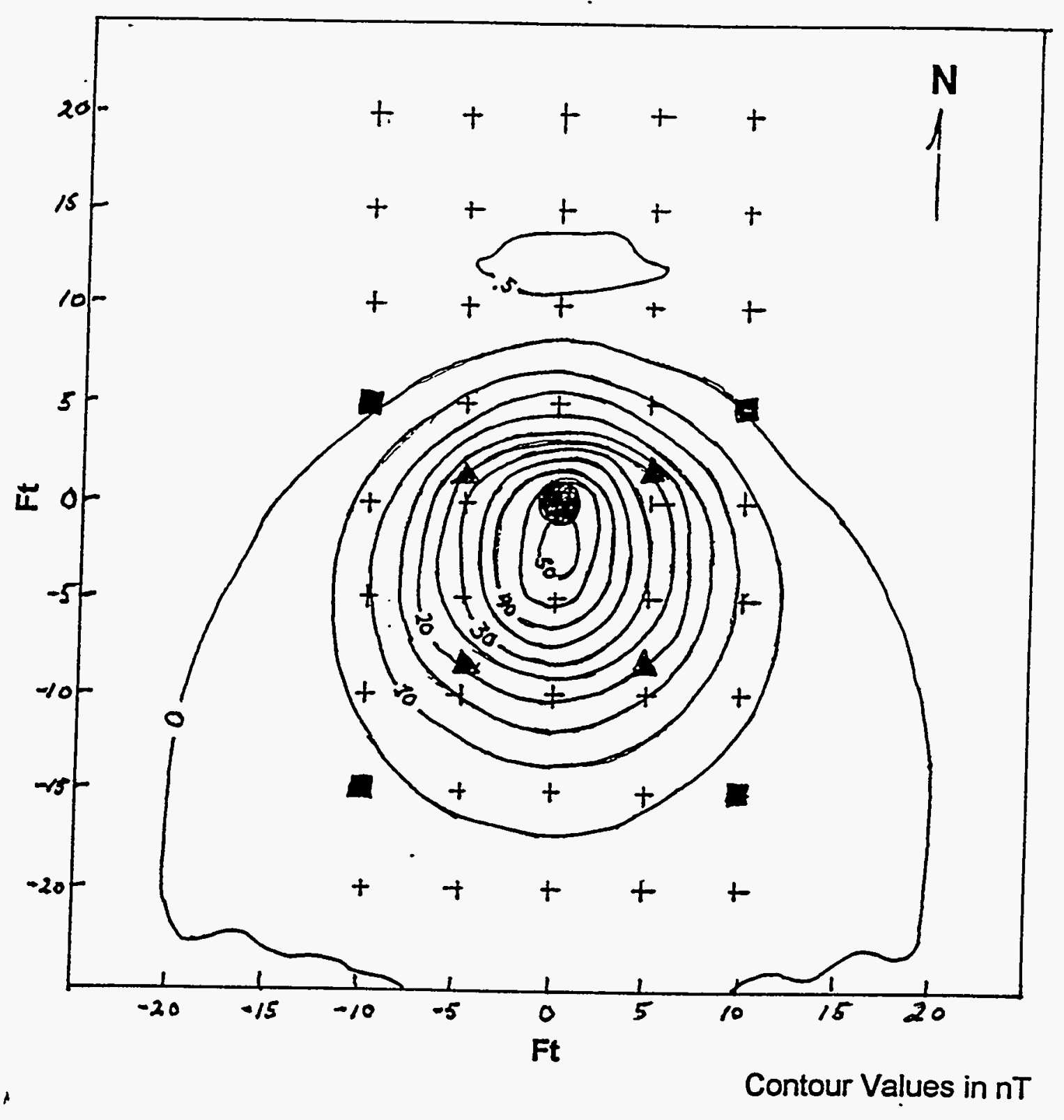

Drum Location

+ $5 \mathrm{Ft}$ Grid Nodes

$\Delta 10 \mathrm{Ft}$ Grid Nodes

20 Ft Grid Nodes

fig. $\$ 10$ Maymetic Survy 


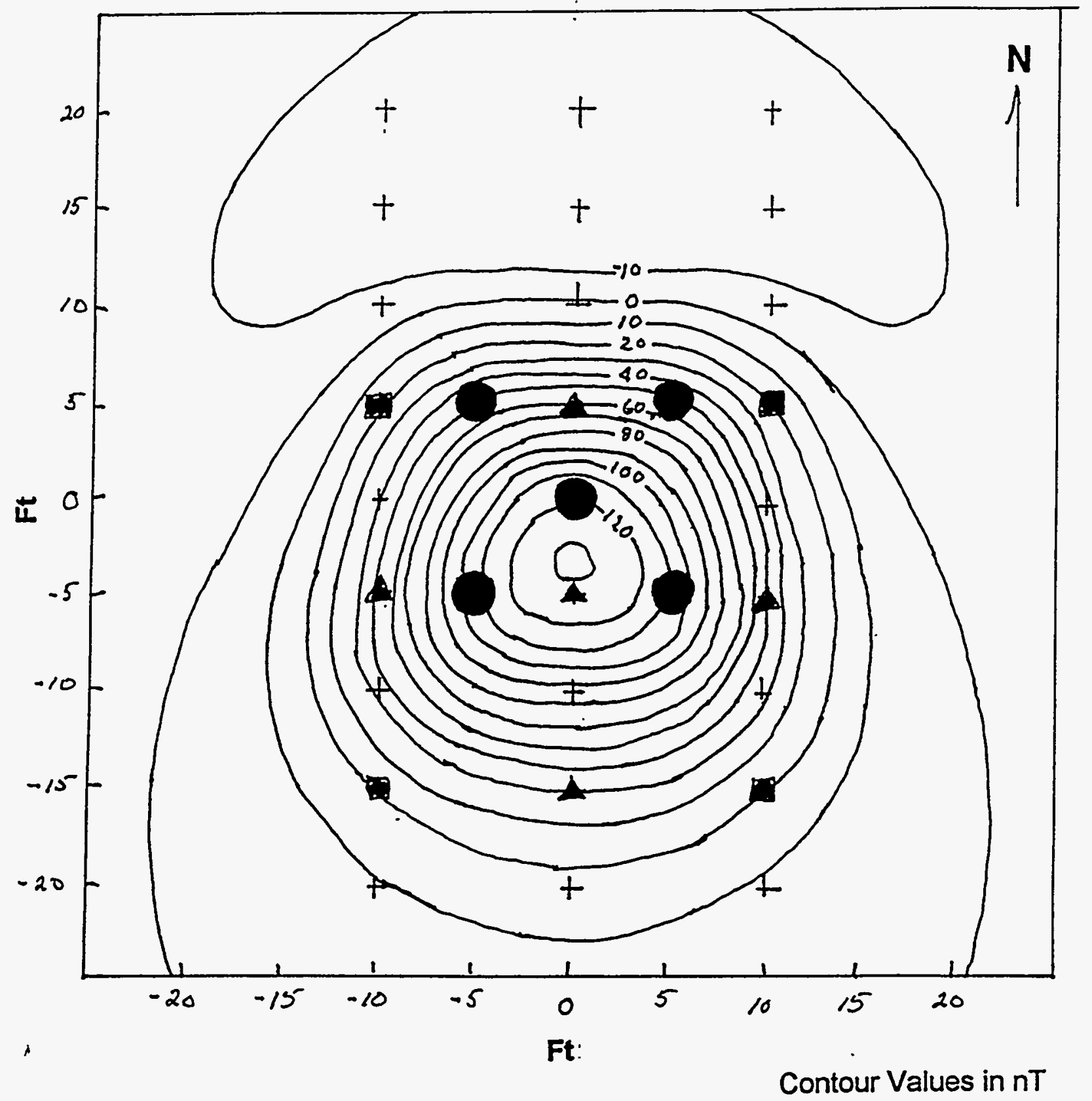

- Drum Location

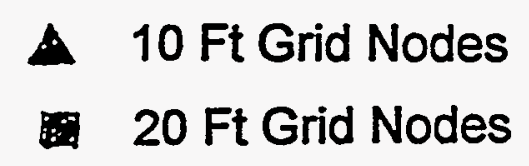

Fag.to.ll Burma Rosd Magnetre Surveg 


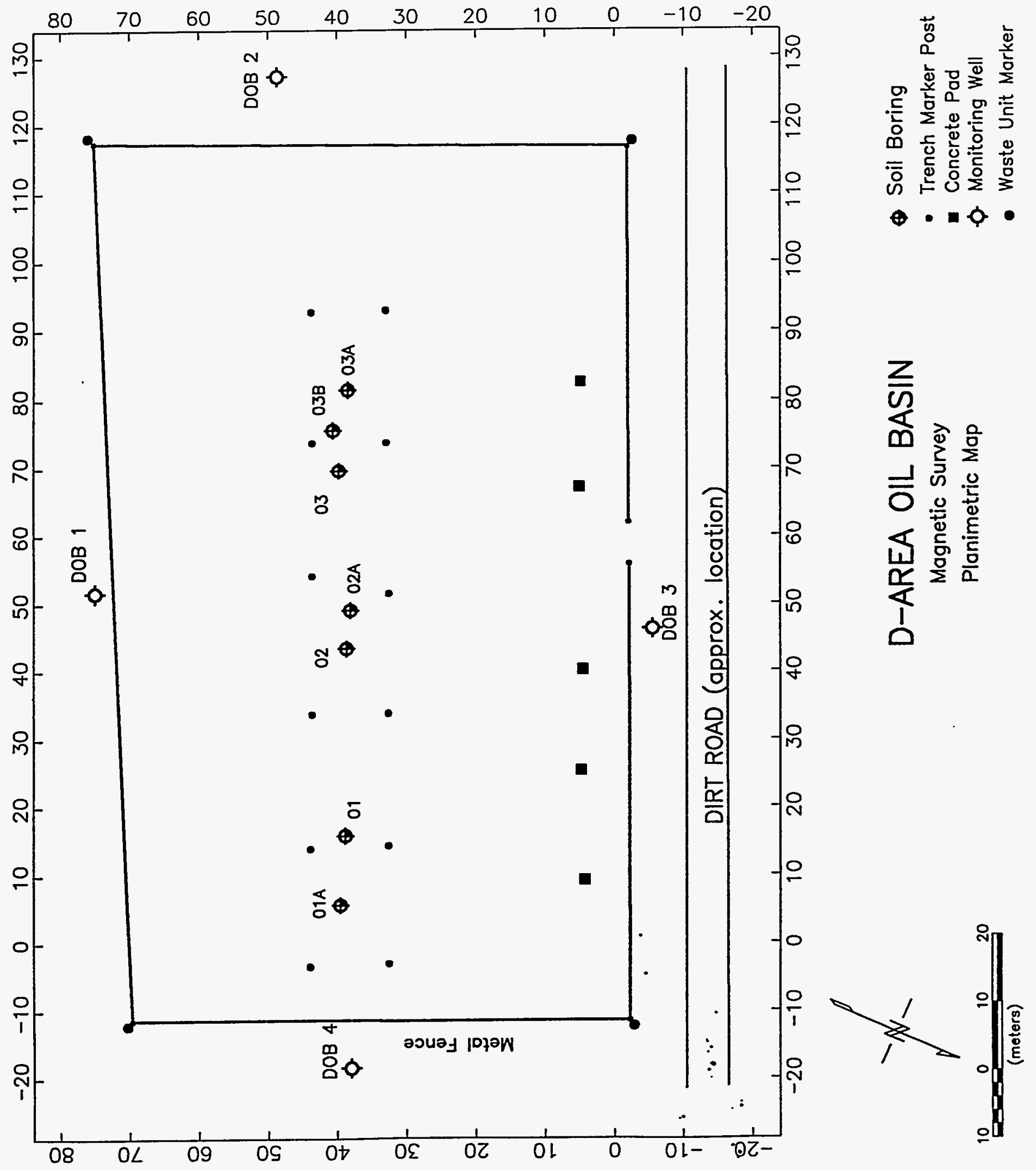



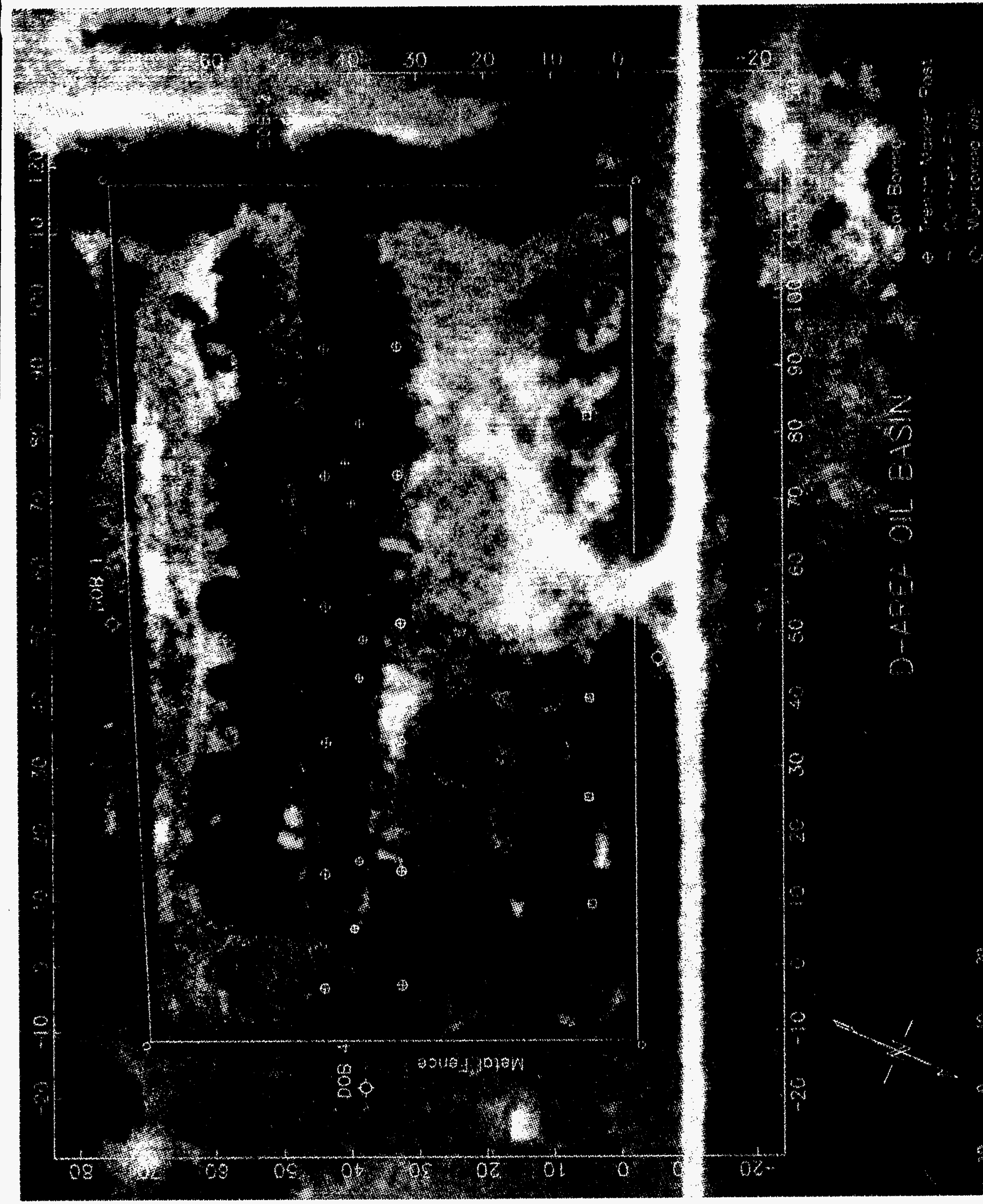


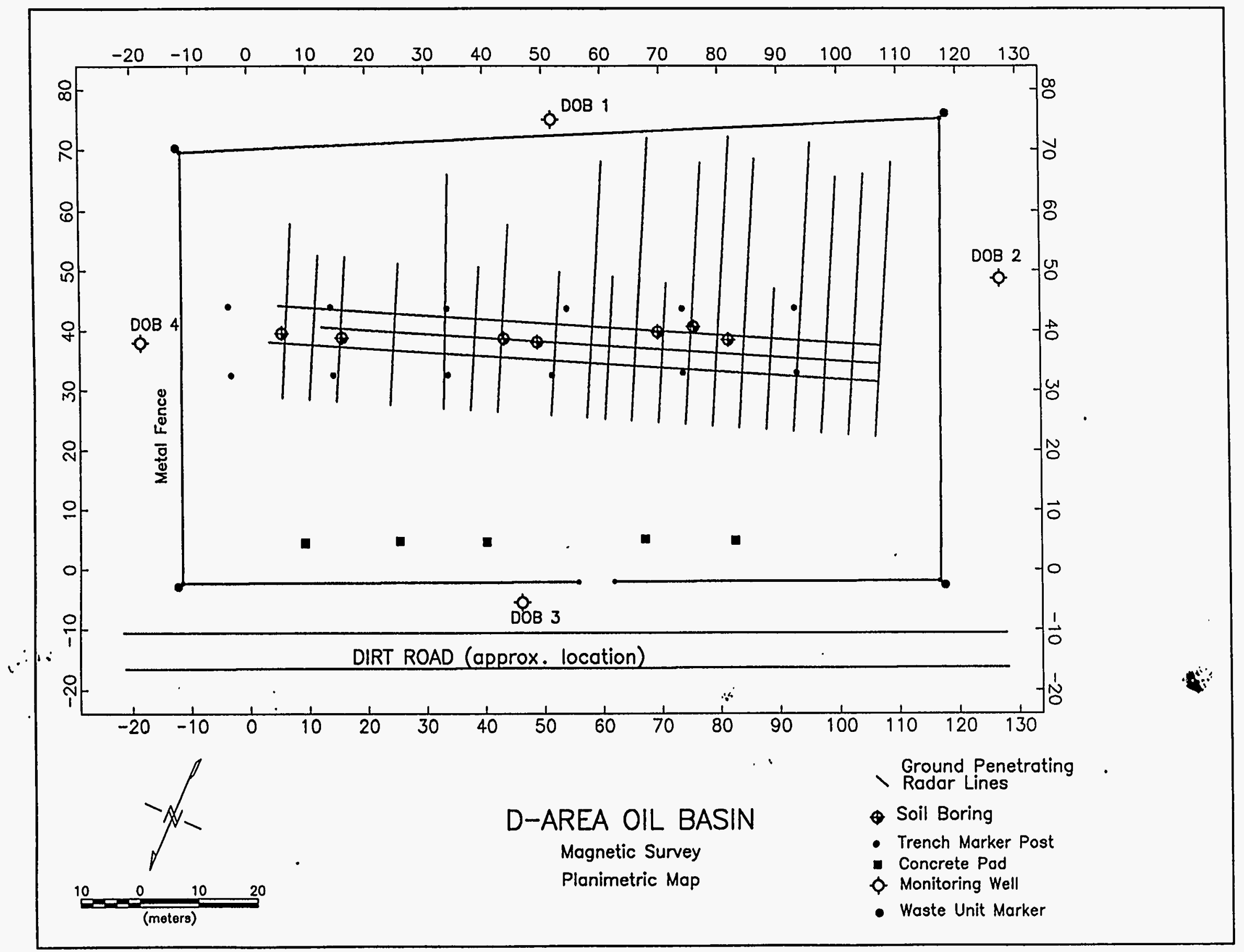




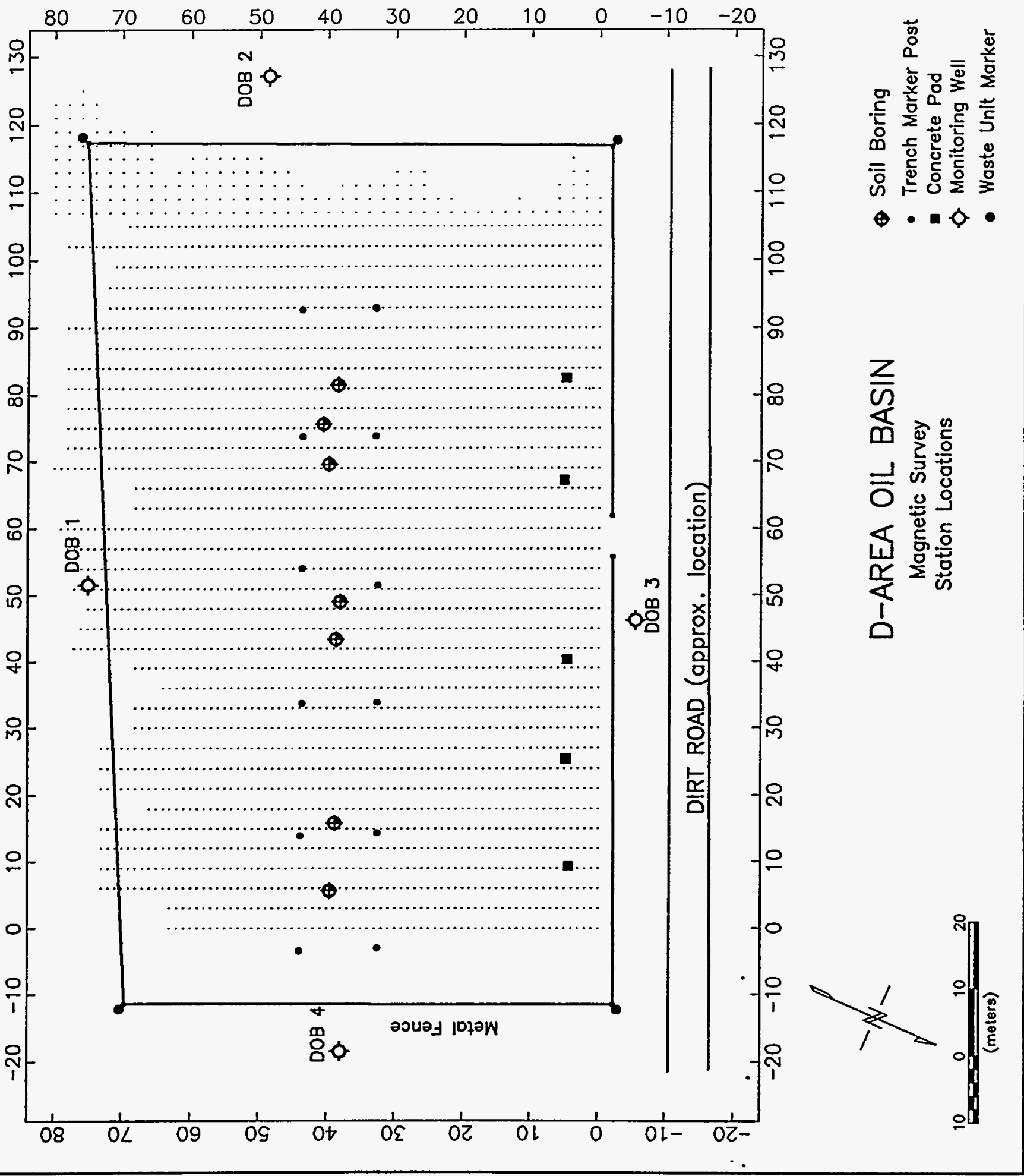




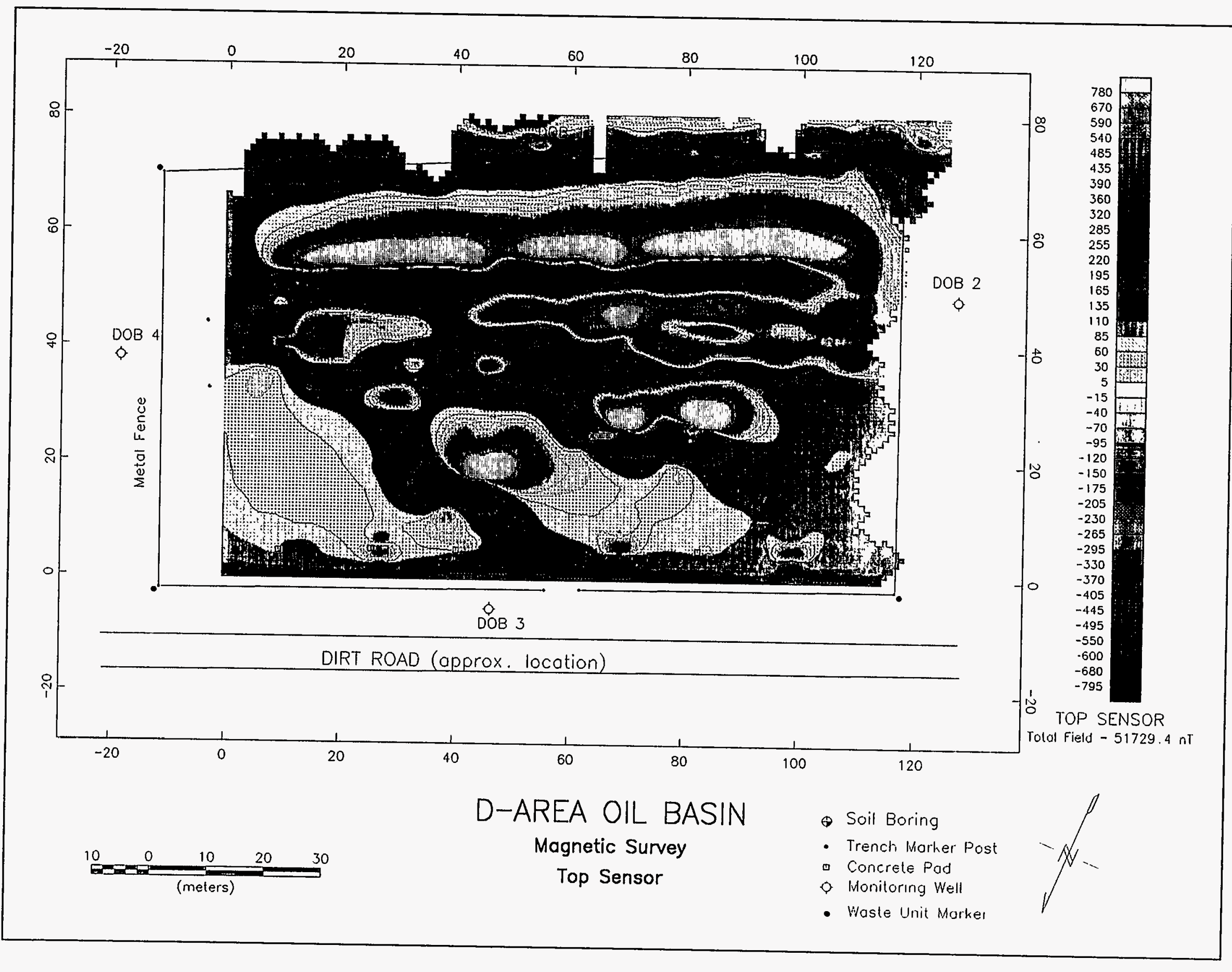




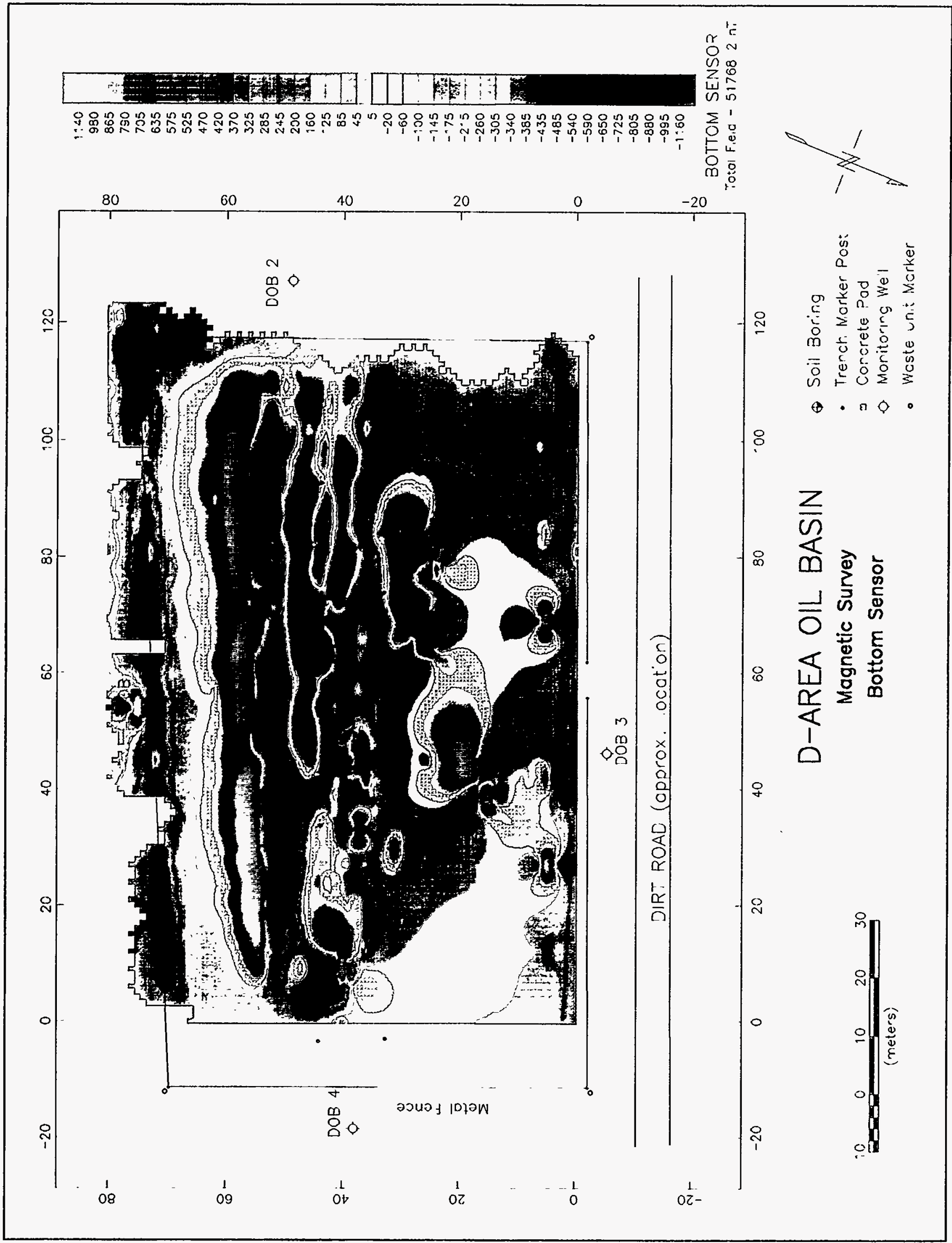




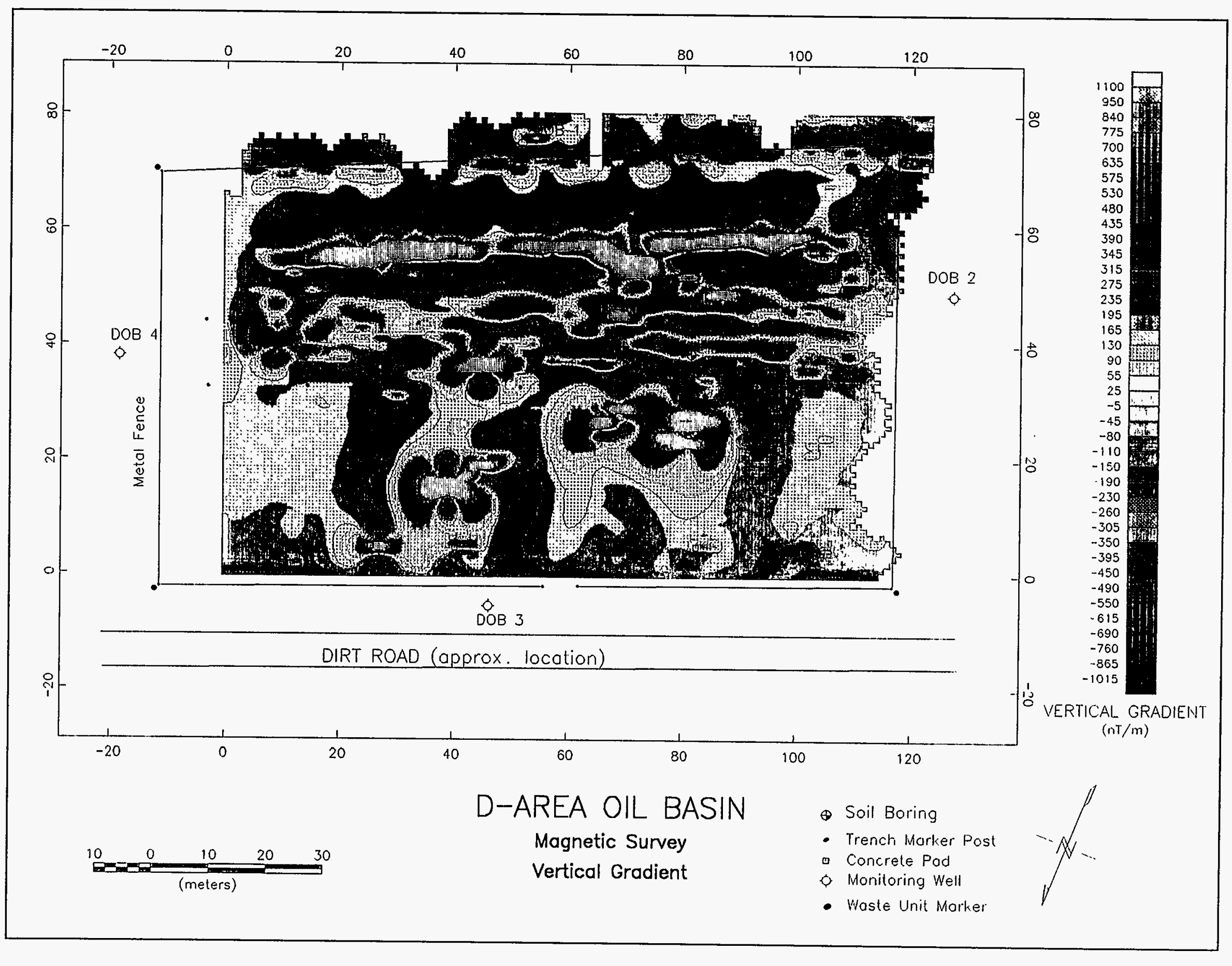


7)

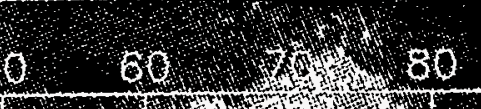
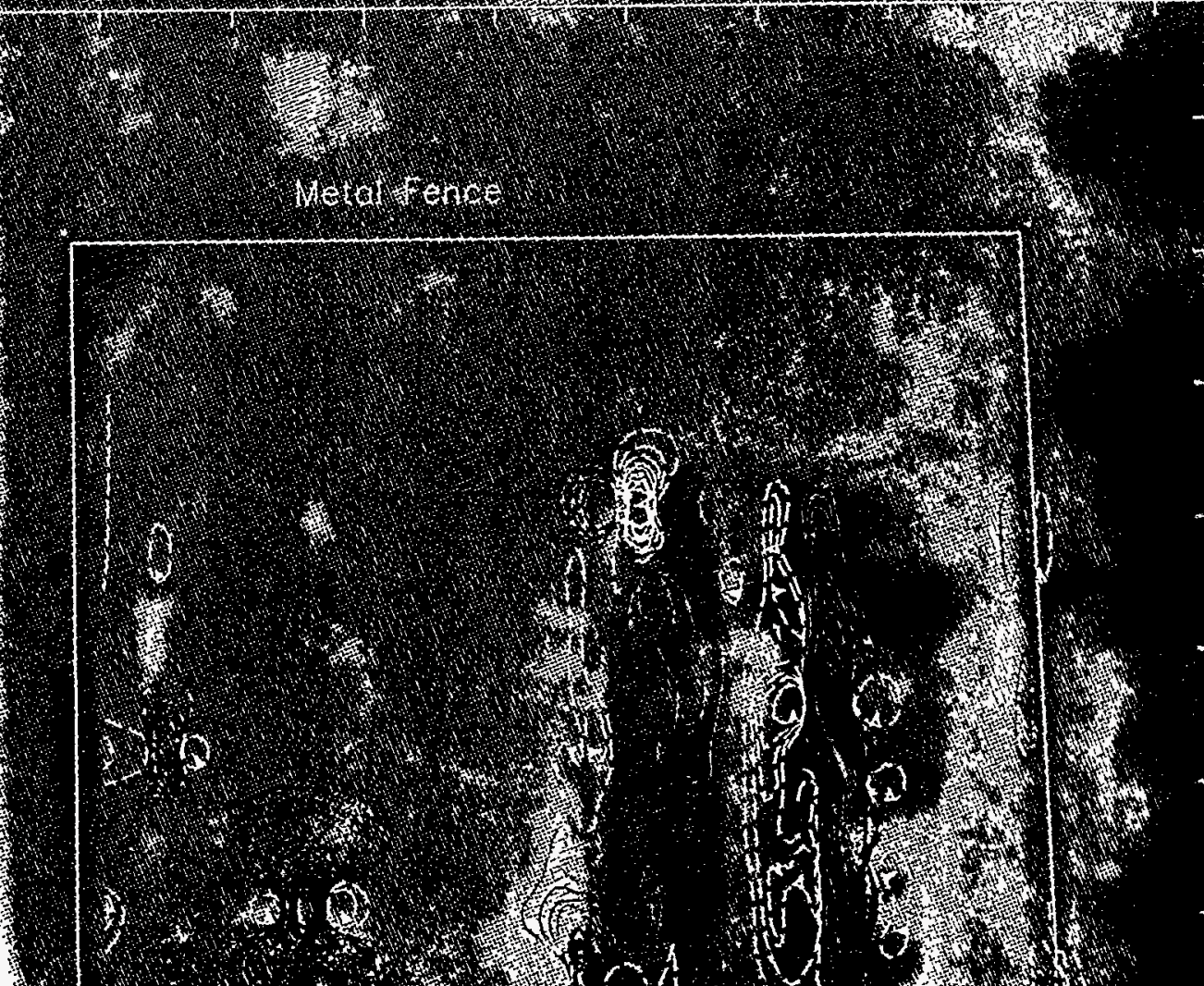

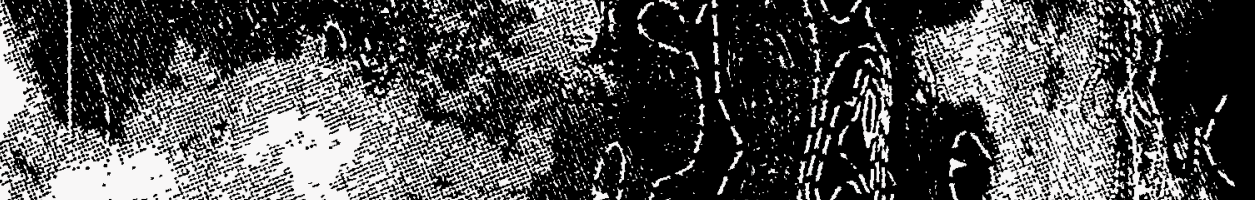

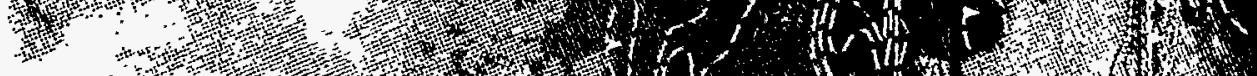
${ }_{3}$ (1) $-1$ (1) W.

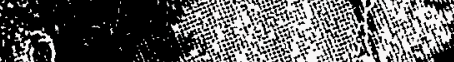
$\$$

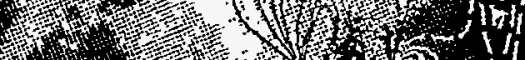

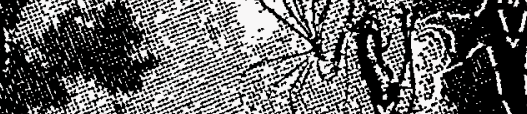

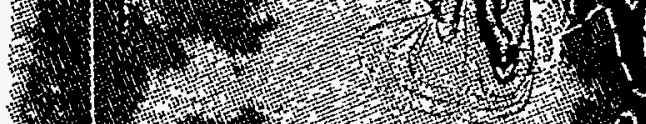

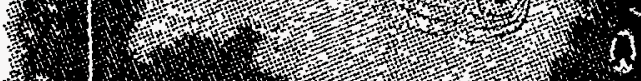
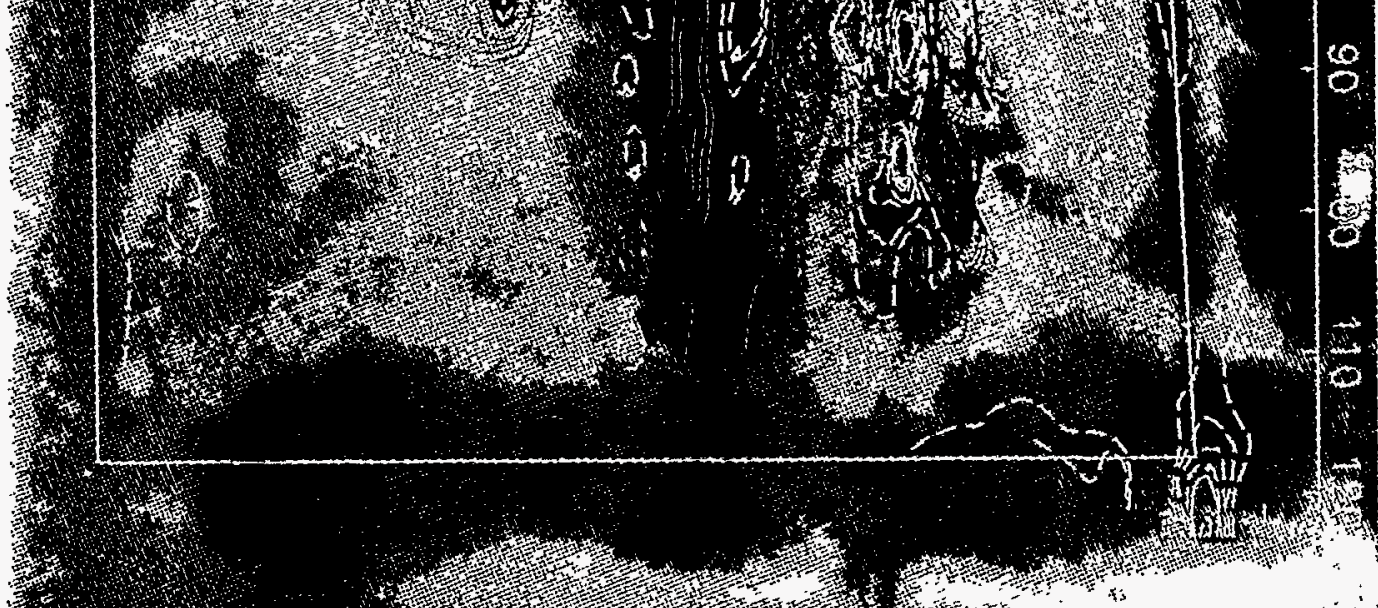

$11,100+10$

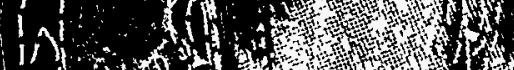

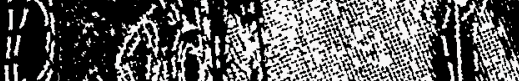

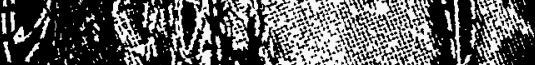

8

1)

7.

(1)

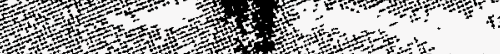

DOW,

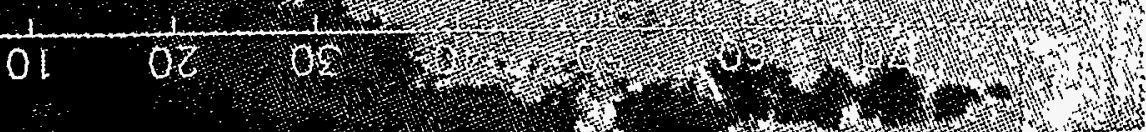


$\sum_{\infty}^{\infty}$

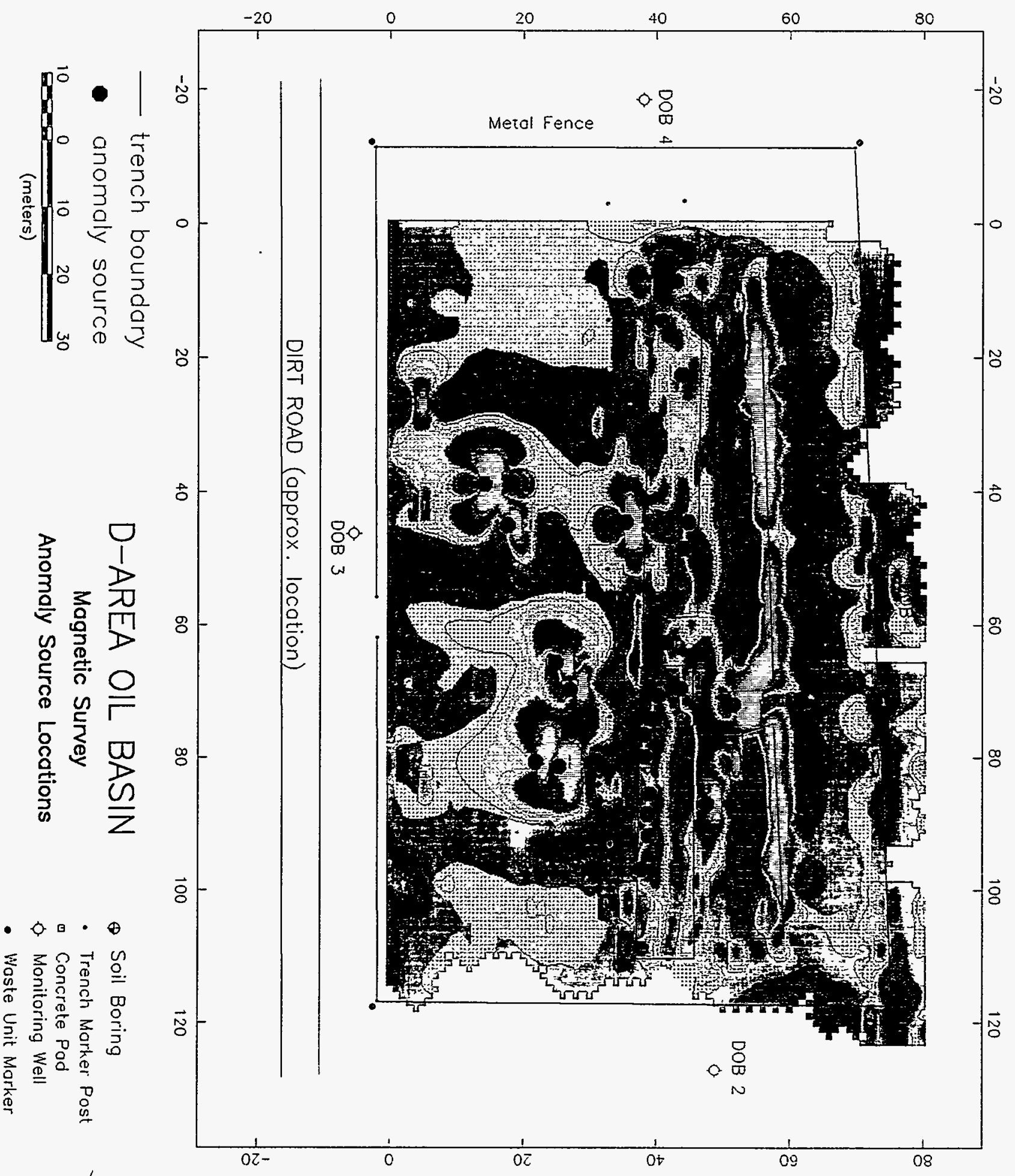

$\gtrless_{1}^{\prime}$ 